\title{
Comparison of intestinal APC subsets from homeostatic and immunostimulatory environments
}

\author{
Kerry Hilligan
}

2014

A thesis submitted to the Victoria University of Wellington in fulfilment of the requirements for the degree of Master of Biomedical Science
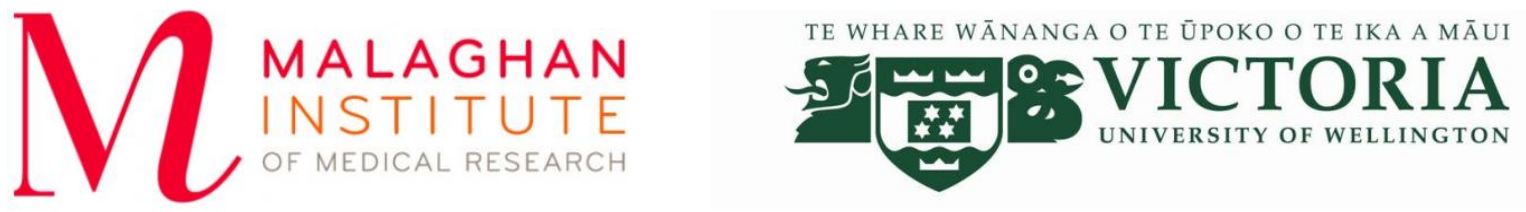


\section{Abstract}

Antigen presenting cells (APC) including dendritic cells (DC) play a key role in the initiation and direction of adaptive immune responses. Acting as sentinels in the tissue, DC sample antigen and traffic to the local lymph node where they present antigen to naïve $\mathrm{T}$ cells. The signals DC provide to naïve T cells determines the functional fate of the $\mathrm{T}$ cell and therefore, the type of immune response generated.

At mucosal sites, such as the intestine, immune responses need to be carefully regulated due to the high antigenic load. For this reason, intestinal immune cells are highly specialised to prevent immune activation to innocuous antigens while still holding the capacity to induce potent responses to pathogenic microbes and helminths. Oral administration of antigen is associated with tolerance and the generation of FoxP3+ regulatory $\mathrm{T}$ cells (Tregs). Specialised lamina propria (LP) resident APC are required for the initiation of Treg differentiation in the mesenteric lymph nodes (MLN) through production of chemical mediators such as retinoic acid (RA). Ablation of these populations or restricted trafficking prevents the development of Tregs in mouse models thus supporting the essential role of APC in maintaining intestinal homeostasis.

During infection, APC promote the induction of adaptive immune responses which neutralise threats. However, the APC subsets involved in this are not well defined. Pathologies such as food allergy and inflammatory bowel disease are thought to arise due to the development of aberrant immune responses. Food allergy can be modelled in mice using the mucosal adjuvant cholera toxin (CT) which has been shown to drive immunity to codelivered antigens and is associated with the generation of IL-4 producing T helper 2 cells. Understanding the APC subsets involved in the initiation of intestinal immune responses could help in the development of targeted therapies for inflammatory bowel conditions.

In this thesis, I show that oral administration of CT is followed by the appearance of a novel phenotype of DC in the intestinal LP and MLN. These DC differ functionally from DC at steady-state and may contribute to the generation of IL-4 producing T cells observed in the LP, MLN and spleen following oral administration of CT. 


\section{Acknowledgements}

First and foremost, I would like to thank my supervisor and mentor Dr. Lisa Connor for being a constant source of support, encouragement and patience throughout my work on this Master's project. From teaching me lab techniques to scientific writing, this thesis would not have been possible without her help - thank you very much! Secondly, I would like to thank my co-supervisor Prof. Franca Ronchese for giving me the opportunity to work on this project in her lab and for being a never-ending source of information and guidance.

I am very grateful to Evelyn Hyde, Shaiu-Choot Tang and Sayani Ghosh for helping out on many big harvest days and to all the other members of the Immune Cell Biology group for advice and assistance. I would also like to thank Dr. Elizabeth Forbes-Blom, Karmella Naidoo and Catherine Plunkett for offering their advice and generously sharing reagents!

I would like to acknowledge and thank all the technical staff at the Malaghan Institute for their expertise and assistance. In particular, thanks to the Biomedical Research Unit staff for expert animal husbandry and sharing their tea room. Also thanks to the Cell Technology Suite staff: Kylie Price, Alfonso (Poncho) Schmidt and Brigitta Mester for their constant support with flow cytometry.

Thank you to all the members of Patagonia over the years. Dr. Deepa Patel and Tina Nie, you were a great sounding board for my various experimental ideas. Thanks to Amy Shepherd for proof-reading this thesis and to Apii for always having a big smile and friendly greeting!

I could not have completed this work without project funding from the Health Research Council of New Zealand and personal funding from the Stewart Charitable Trust and Victoria University of Wellington. Thank you very much.

I could not have done this without the support of my fantastic friends and family. $\mathrm{J}$ and $\mathrm{T}$, thanks for all the friendly greetings and companionship throughout my study. Thanks to my 
lovely flatmates for putting up with my absences and missed BYOs! Also, thank to everyone at AM Wellington for offering me friendship and a shoulder to lean on. I am truly grateful to M.T. for supporting me through the writing of this thesis and for being a whizz with the formatting! And a special thank you to Grandpa who inspired my love of learning and science!

Last but not least, Mum, Dad and Michael - sorry for the grumpiness and stress you have put up with over the last two and a half years. All the cups of tea, dinners and constant words of encouragement really made a huge difference. You are stars!!! 


\section{Table of contents}

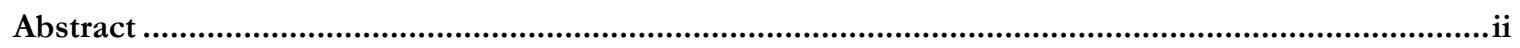

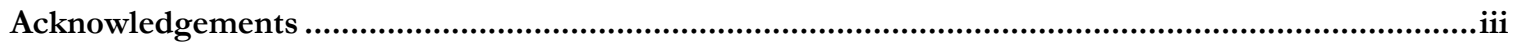

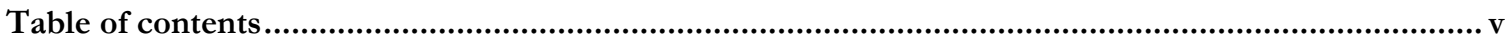

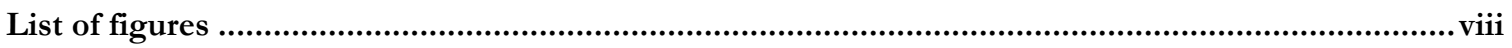

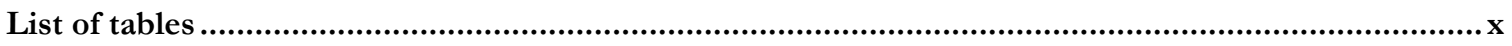

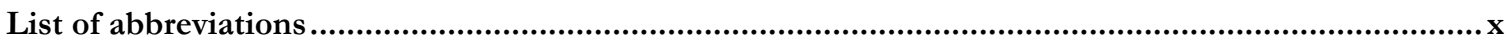

CHAPTER 1: GENERAL INTRODUCTION ...............................................1

1.1. APC: the link between innate and adaptive immunity ................................................

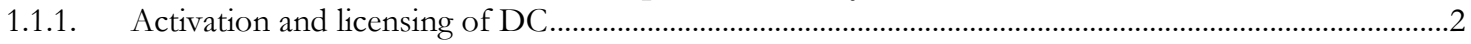

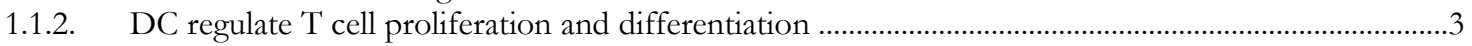

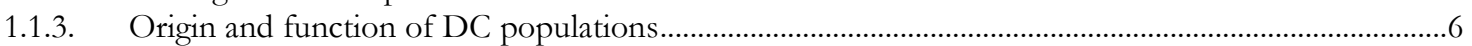

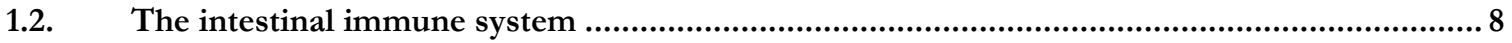

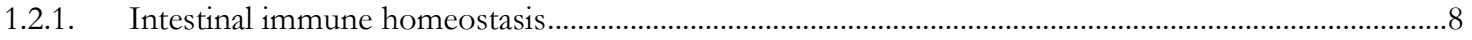

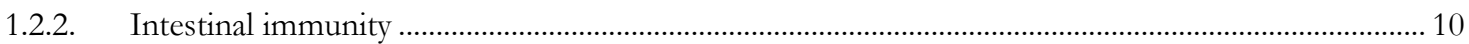

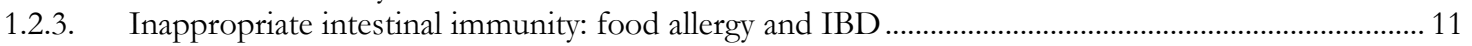

1.2.4. Intestinal APC hold the key to controlling aberrant immune responses ............................................ 12

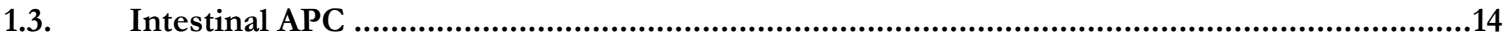

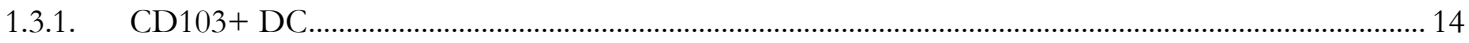

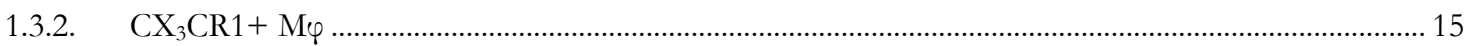

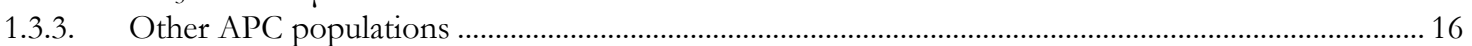

1.4. APC in the regulation of intestinal immune responses .................................................19

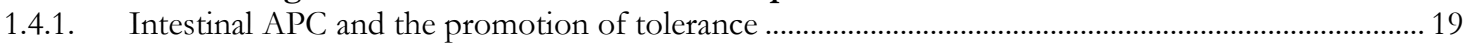

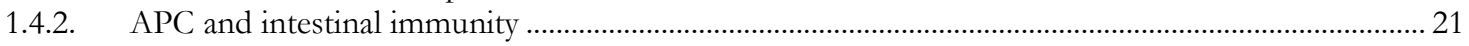

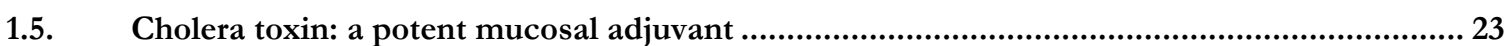

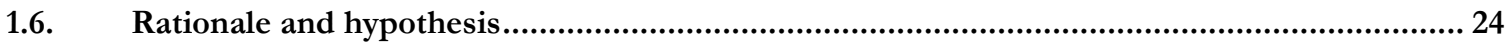

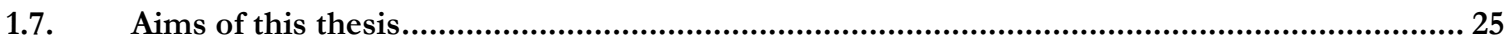

CHAPTER 2: MATERIALS AND METHODS...............................................26

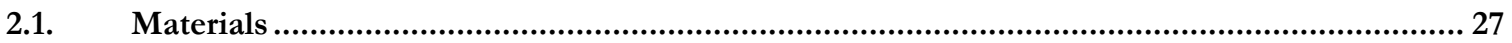

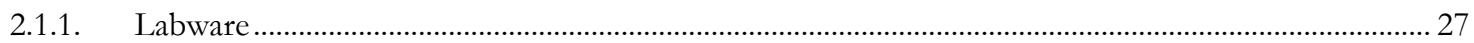

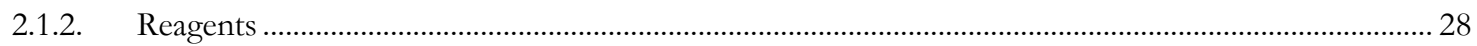

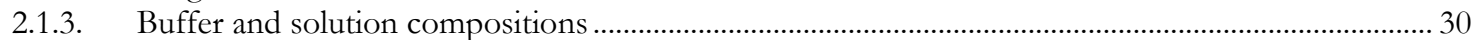

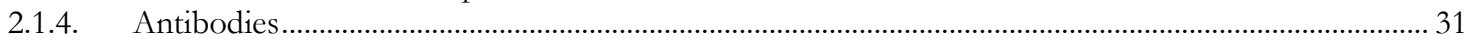

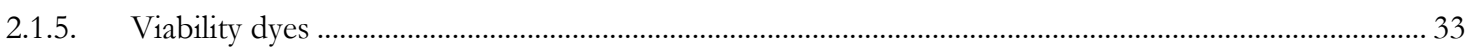

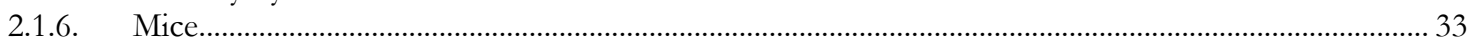




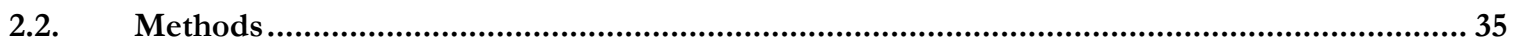

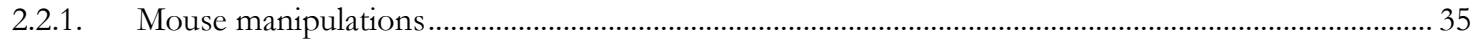

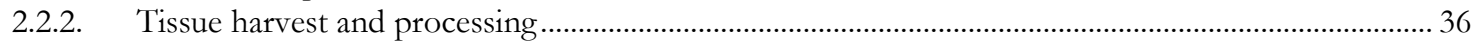

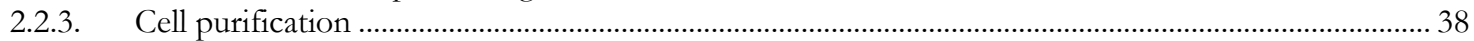

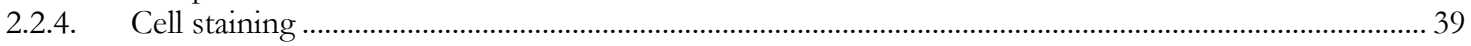

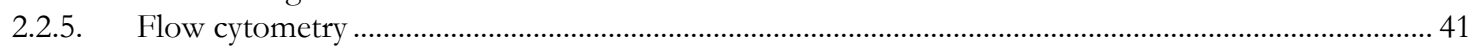

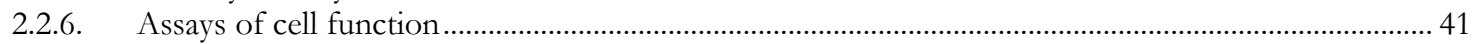

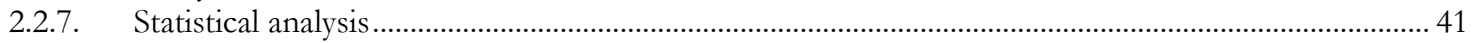

\section{CHAPTER 3: PHENOTYPIC CHARACTERISATION OF INTESTINAL APC} SUBSETS AT STEADY-STATE ............................................42

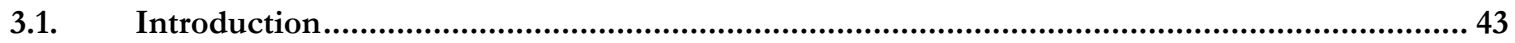

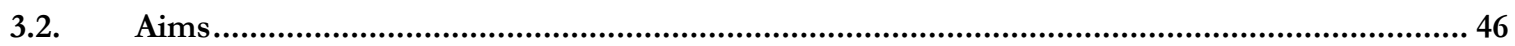

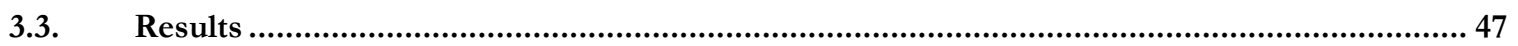

3.3.1. LP APC phenotype and subset compositions at steady-state .......................................................... 47

3.3.2. CD103 and TIM-4 expression clearly defines three populations of CD11b+ APC in the LP at

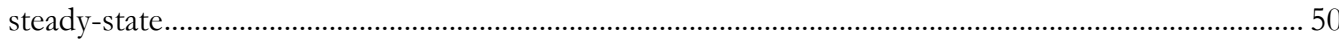

3.3.3. CD103- TIM-4+ APC take up antigen at steady-state ........................................................................... 55

3.3.4. CD103+ CD11b+ LP APC have high activity of RALDH at steady-state......................................... 57

3.3.5. MLN DC phenotype and subset compositions at steady-state............................................................. 59

3.3.6. MHCIIhi DC from the MLN have a high frequency of cells expressing active RALDH .................. 62

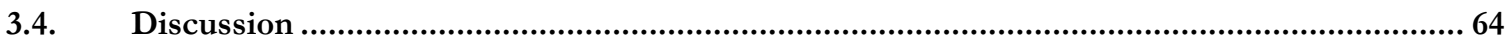

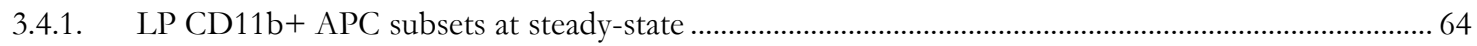

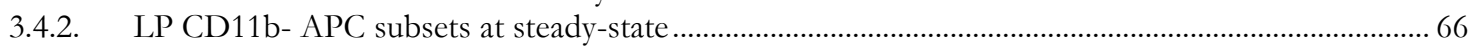

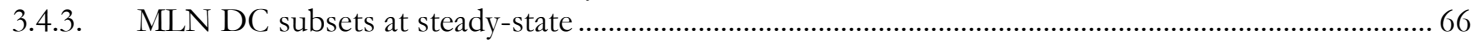

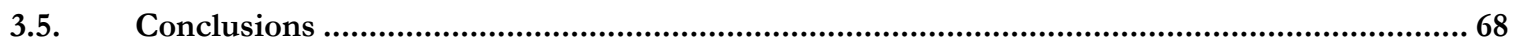

\section{CHAPTER 4: PHENOTYPIC CHARACTERISATION OF INTESTINAL APC SUBSETS FOLLOWING ORAL ADMINISTRATION OF A MUCOSAL ADJUVANT ....................................................69}

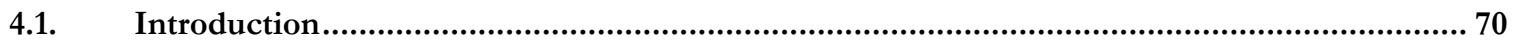

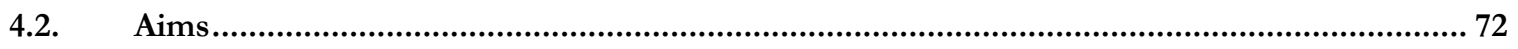

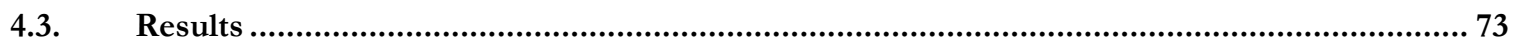

4.3.1. Oral administration of cholera toxin changes the composition of APC subsets in the LP and

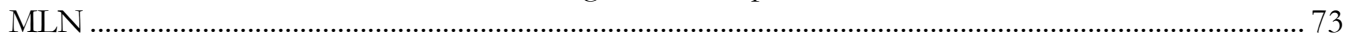

4.3.2. CT is sufficient to induce the appearance of CD103+ TIM-4+ DC in the intestine ........................... 77

4.3.3. CD103+ TIM-4+ cells appear transiently in the intestine following OVA+CT treatment ..................77

4.3.4. CT reduces expression of $\mathrm{CX}_{3} \mathrm{CR} 1$ but not $\mathrm{F} 4 / 80$ or $\mathrm{CD} 64$ on CD103- APC .................................. 79

4.3.5. CD103+ TIM-4+ cells are phenotypically similar to CD103+ TIM-4- DC ………………………….. 79

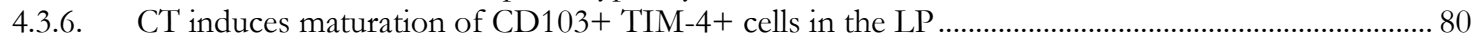

4.3.7. CD103+ TIM-4- and CD103+ TIM-4+ cells express active RALDH ……………............................ 82

4.3.8. TLR4 signalling is not required for the increased frequency of CD103+ TIM-4+ cells in the LP and MLN following oral administration of CT …………………………………………………....... 84

4.3.9. The increase in frequency of CD103+ TIM-4+ cells following oral CT is independent of intestinal commensal microbes 


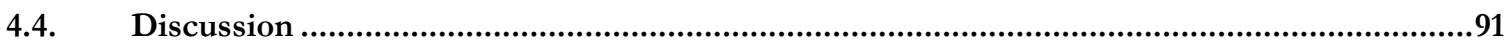

4.4.1. CD103+ DC upregulate TIM-4 in response to CT ….......................................................................... 91

4.4.2. CT induced TIM-4 upregulation by CD103+ DC does not require signalling through TLR4 or

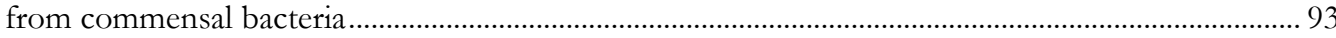

4.4.3. CD103+ TIM-4+ DC are present in the lymph node ......................................................................... 94

\section{CHAPTER 5: ORALLY ADMINISTERED CHOLERA TOXIN LEADS TO FUNCTIONAL CHANGES AMONG INTESTINAL IMMUNE CELLS .........................................................................96}

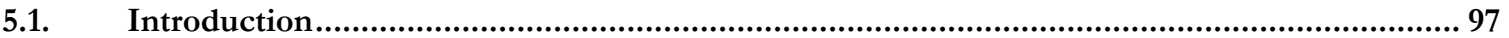

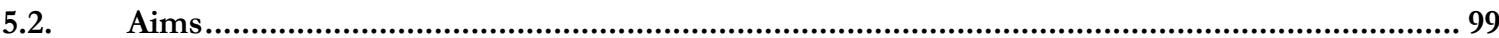

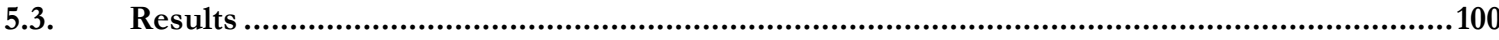

5.3.1. $\quad$ OVA+CT induces antigen specific $\mathrm{T}$ cell proliferation and activation ............................................. 100

5.3.2. CT increases the frequency of IL-4 producing T cells in the LP, MLN and spleen ........................ 100

5.3.3. CT enhances antigen specific $\mathrm{T}$ cell proliferation but not activation.................................................. 104

5.3.4. T cell derived IFN $\gamma$ and IL-4 could not be detected by ICS following CT treatment .....................107

5.3.5. RALDH activity does not change following oral administration of CT ........................................... 109

5.3.6. CD103+ TIM-4+ DC show an increased capacity to take up apoptotic cells compared to CD103+ TIM-4- DC ex vivo...

5.3.7. TIM-4 upregulation by CD103+ DC modestly increases antigen uptake capacity .......................... 114

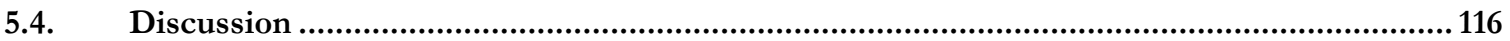

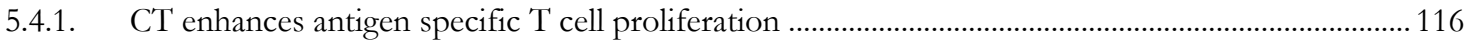

5.4.2. CT increases the frequency of IL-4 producing $\mathrm{T}$ cells in the intestine and spleen............................117

5.4.3. CT does not affect RALDH activity among LP and MLN APC ....................................................... 118

5.4.4. CD103+ TIM-4+ DC have an increased capacity for apoptotic cell uptake ex vivo compared to

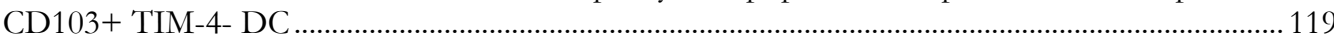

5.4.5. CD103+ TIM-4+ DC have a modest increase in capacity for antigen uptake in vivo compared to CD103+ TIM-4- DC ....................................................................................................................... 119

5.5. Conclusions

CHAPTER 6: GENERAL DISCUSSION …....................................................121

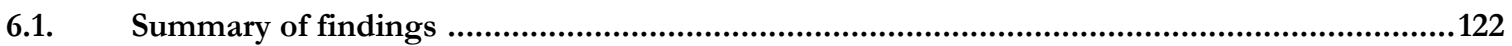

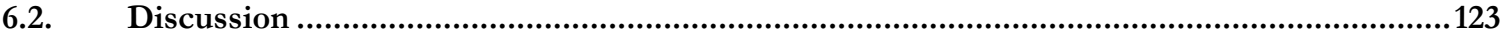

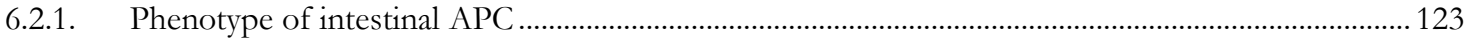

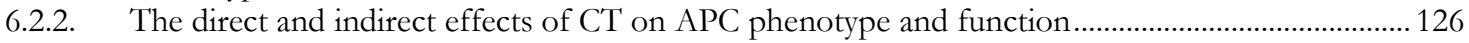

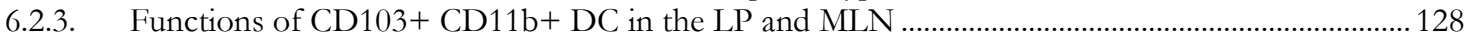

6.2.4. Functions of monocyte-derived APC in the LP and MLN ............................................................ 131

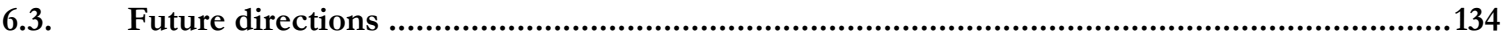

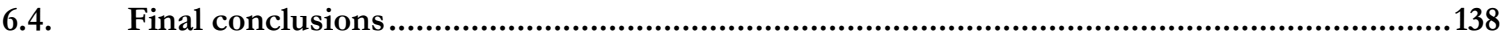

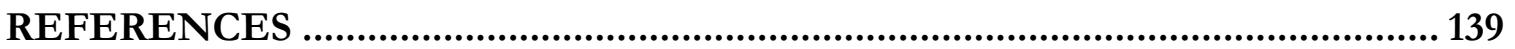

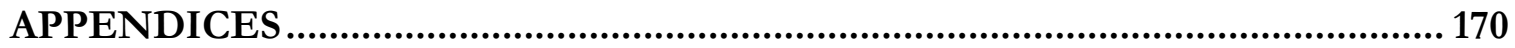




\section{List of figures}

Figure 1.1: DC direct $\mathrm{T}$ cell proliferation and differentiation ....................................................

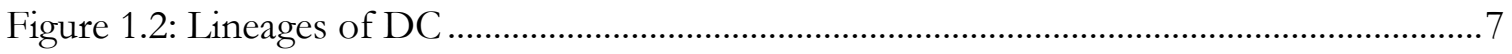

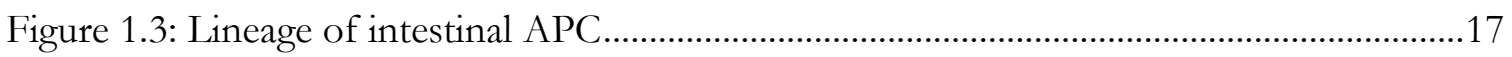

Figure 1.4: Intestinal APC regulate oral tolerance ……...............................................................20

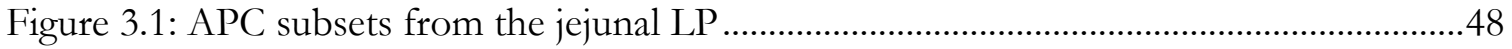

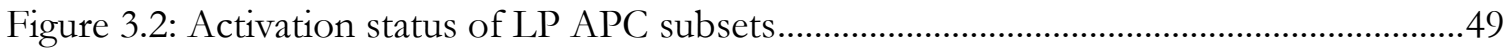

Figure 3.3: Cell surface marker expression on LP APC ...........................................................52

Figure 3.4: CD103- CD11b+ APC can be divided into two distinct populations ....................54

Figure 3.5: CD11b+ TIM-4+ APC take up antigen at steady-state ...........................................56

Figure 3.6: CD103+ LPDC have active RALDH at steady-state ................................................58

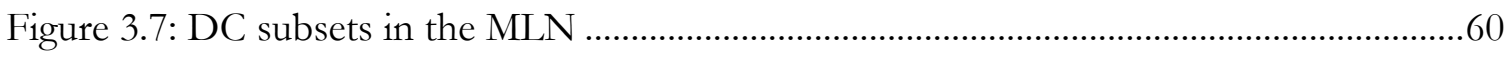

Figure 3.8: Cell surface markers on DC in the MLN.................................................................61

Figure 3.9: CD103+ DC in the MLN have active RALDH at steady-state ..............................63

Figure 4.1: CT induces the appearance of a novel phenotype of APC in the LP.....................75

Figure 4.2: CT increases the frequency of CD103+ CD11b+ TIM-4+ APC in the MLN ....76

Figure 4.3: CT induces the appearance of CD103+ TIM-4+ APC in the LP and MLN.......78

Figure 4.4: CD103+ TIM-4+ APC appear transiently in the LP and MLN following CT

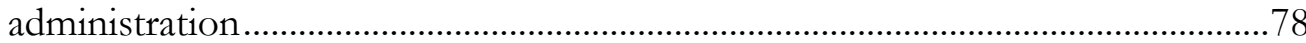

Figure 4.5: CD103+ TIM-4+ APC show increased activation and lack markers of M $\varphi$ lineage.

Figure 4.6: CD103+ TIM-4+ APC share morphological characteristics of CD103+ TIM-4- DC and CD103+ TIM-4+ M $\varphi$. .82

Figure 4.7: CD103+ TIM-4- and CD103+ TIM-4+ APC show high RALDH activity

Figure 4.8: TLR4 is not required for the appearance of CD103+ TIM-4+ APC in the LP...85

Figure 4.9: TLR4 is not required for the appearance of CD103+ TIM-4+ APC in the MLN

Figure 4.10: DSS induced colitis does not change small intestinal APC subset compositions significantly 
Figure 4.11: Commensal microbes are not required for the appearance of CD103+ TIM-

$4+\mathrm{APC}$ in the LP

Figure 4.12: Commensal microbes are not required for the appearance of CD103+ TIM-

4+ APC in the MLN

Figure 5.1: Experimental protocol used to analyse antigen specific $\mathrm{T}$ cell responses following oral administration of OVA+CT.

Figure 5.2: CT increases the frequency and activation of antigen specific T cells in the LP and MLN. 102

Figure 5.3: CT increases the frequency of IL-4 producing antigen specific T cells.

Figure 5.4: Experimental protocol used to analyse antigen specific T cell responses by intracellular cytokine staining following oral administration of OVA+CT. ..... 105

Figure 5.5: CT enhances antigen specific T cell expansion in the MLN and spleen. .106

Figure 5.6: Antigen specific cytokine production could not be detected by ICS following oral OVA+CT treatment

Figure 5.7: CT treatment does not change RALDH activity among intestinal APC populations

Figure 5.9: CD103+ TIM-4+ show an increased capacity for apoptotic cell uptake compared to CD103+ TIM-4- DC.

Figure 5.8: Dexamethasone induces apoptosis in thymocytes and CD11c+ positive magnetic enrichment preferentially enriches for CD103+ DC in the LP and MLN

Figure 5.10: TIM-4 upregulation by CD103+ DC modestly increases antigen uptake capacity in vivo.

Figure 6.1: Proposed mechanisms by which CT treatment promotes phenotypic and functional changes among intestinal APC and T cells.

Appendix I: Gating strategy used to identify APC subsets in the jejunal LP........................ 171

Appendix II: Gating strategy used to identify APC subsets in the MLN ............................... 172 


\section{List of tables}

Table 1: Summary of cell surface markers expressed by intestinal APC populations...............18

Table 2: Summary of the phenotype and function of intestinal APC from mice and humans

\section{List of abbreviations}

$\begin{array}{ll}\text { APC } & \text { antigen presenting cell } \\ \text { BMDC } & \text { bone marrow derived dendritic cell } \\ \text { BSA } & \text { bovine serum albumin } \\ \text { CCL } & \text { chemokine ligand } \\ \text { CCR } & \text { chemokine receptor } \\ \text { CD } & \text { cluster of differentiation } \\ \text { CT } & \text { cholera toxin } \\ \text { CX }{ }_{3} \text { CL1 } & \text { CX3C ligand 1 (fractalkine) } \\ \text { CX }{ }_{3} \text { CR1 } & \text { CX }{ }_{3} \text { C chemokine receptor 1 (fractalkine receptor) } \\ \text { DAMPs } & \text { danger associated molecular patterns } \\ \text { DC } & \text { dendritic cell } \\ \text { DSS } & \text { dextran sulfate sodium } \\ \text { FMO } & \text { fluorescence minus one } \\ \text { FoxP3 } & \text { forkhead box protein 3 } \\ \text { ICS } & \text { intracellular cytokine staining } \\ \text { IFN } & \text { interferon } \\ \text { Ig } & \text { immunoglobulin } \\ \text { IL } & \text { interleukin } \\ \text { IRF } & \text { interferon regulatory factor } \\ \text { LP } & \text { lamina propria } \\ \text { LPS } & \text { lipopolysaccharide } \\ \text { MAMPs } & \text { microbial associated molecular patterns } \\ \text { MFI } & \text { median fluorescence intensity } \\ & \end{array}$




$\begin{array}{ll}\text { MHCI } & \text { major histocompatibility complex class I } \\ \text { MHCII } & \text { major histocompatibility complex class II } \\ \text { MLN } & \text { mesenteric lymph node } \\ \text { MoDC } & \text { monocyte-derived dendritic cell } \\ \text { M } \varphi & \text { macrophage } \\ \text { OVA } & \text { ovalbumin } \\ \text { PP } & \text { Peyer's patch } \\ \text { PRR } & \text { pattern recognition receptor } \\ \text { PtdSer } & \text { phosphatidylserine } \\ \text { RA } & \text { retinoic acid } \\ \text { RALDH } & \text { retinal dehydrogenase } \\ \text { TGF } & \text { transforming growth factor } \\ \text { Th } & \text { Thelper cell } \\ \text { TIM } & \text { T cell immunoglobulin and mucin domain containing protein } \\ \text { TLR } & \text { toll like receptor } \\ \text { TNF } & \text { tumour necrosis factor } \\ \text { Treg } & \text { T regulatory cell } \\ \text { TSLP } & \text { thymic stromal lymphopoietin }\end{array}$

\section{Routes of injection}

$\begin{array}{ll}\text { i.g. } & \text { intragastric (by oral gavage) } \\ \text { i.p. } & \text { intraperitoneal } \\ \text { i.v. } & \text { intravenous }\end{array}$




\section{Chapter 1: General Introduction}




\subsection{APC: the link between innate and adaptive immunity}

The two arms of the immune system play very different but nevertheless, essential roles to host defence. The key regulators of cross-talk between the innate and adaptive systems are antigen presenting cells (APC). Dendritic cells (DC) are described as professional APC due to their superior ability to initiate and direct adaptive immune responses compared to other APC such as macrophage $(\mathrm{M} \varphi)$ and $\mathrm{B}$ cells ${ }^{1,2}$. It is now understood that DC are innate immune cells that act as sentinels, monitoring the tissue for danger or pathogen associated motifs. Following acquisition of antigen, DC migrate to the lymph node where they present antigen to T cells and drive $\mathrm{T}$ cell proliferation and differentiation ${ }^{3}$.

The innate immune system provides the first line of defence against pathogens. Cells of the innate immune system are equipped with pathogen recognition receptors (PRR) including toll-like receptors (TLR) and NOD-like receptors (NLR) which enables them to detect signs of microbial invasion. Innate cells respond by producing cytokines, chemokines and antimicrobial proteins in order to drive inflammation, neutralise threats and activate cells of the adaptive immune system ${ }^{4}$.

The adaptive immune system consists of $\mathrm{T}$ and $\mathrm{B}$ lymphocytes which are able to target threats more specifically. Genetic shuffling of antigen receptor components allows for the generation of a repertoire of adaptive immune cells capable of responding to vast numbers of individual antigens. Upon infection, activation of adaptive immunity results in proliferation of highly specific effector cells that can directly kill infected cells or provide cytokines to recruit populations of other immune cells ${ }^{5}$. In addition, following the resolution of the infection, small numbers of these effector cells remain in circulation as memory cells allowing for faster, more effective responses to future infection ${ }^{1}$.

\subsubsection{Activation and licensing of DC}

Primarily the role of DC is to monitor peripheral tissues and sample environmental antigen by phagocytosis and receptor-mediated endocytosis. Antigen is processed and displayed on the cell surface on major histocompatibility complex (MHC) molecules. Exogenous antigen is displayed on MHCII molecules to specifically activate CD4 T cells while endogenous 
antigen is presented on MHCI to CD8 T cells. Importantly, specific subsets of DC are able to cross-present exogenous antigen to CD8 cytotoxic T cells via MHCI molecules ${ }^{6}$.

At steady-state, DC traffic constitutively to the draining lymph node to replenish other DC populations and present antigen to $\mathrm{T}$ cells in order to maintain immunological tolerance to self antigens ${ }^{7}$. On the other hand, sensing of pathogen or danger associated motifs through TLRs and NLRs leads to immune activation ${ }^{6}$. Signalling through PRRs leads to DC activation and licensing through upregulation of co-stimulatory molecules CD80/86 ${ }^{8}$ and lymph node homing receptor CCR7 9 . DC subsequently traffic to the lymph node and interact with $T$ cells promoting the initiation of adaptive immune responses.

\subsection{2. $\quad \mathrm{DC}$ regulate $\mathrm{T}$ cell proliferation and differentiation}

$\mathrm{T}$ cells require three signals from $\mathrm{DC}$ to proliferate and differentiate into appropriate effector cells (Fig. 1.1.). Firstly, DC present antigen on MHC molecules to T cells expressing the $\mathrm{T}$ cell receptor (TCR) specific for that antigen. This signal allows for activation and expansion of the $\mathrm{T}$ cell capable of recognising and inducing the appropriate response to the microbial agent. Secondly, mature DC express increased levels of co-stimulatory molecules, including CD80 and CD86 which bind to CD28 on naïve T cells. This interaction ensures sufficient $\mathrm{T}$ cell activation and prevents permanent $\mathrm{T}$ cell unresponsiveness known as $\mathrm{T}$ cell anergy ${ }^{8,10}$. Finally, environmental cues condition DC to instruct differentiation of T cells to the appropriate effector cell type through production of soluble chemical mediators, such as cytokines, and additional receptor-ligand interactions. Following these signals, naive T cells become effector cells capable of elaborating effector functions, including cytotoxicity, cytokine production and immune suppression. Cytotoxic T cells directly kill infected cells through perforin and/or granzyme mechanisms ${ }^{11,12,13}$ whereas helper $\mathrm{T}$ cells produce cytokines which can promote antibody production by B cells, activation and recruitment of immune cells or inhibit proliferation and activation of an immune response ${ }^{6}$. Both CD4+ and CD8+ T cells have the capacity to adopt either of these functions, however, CD8+ T cells are primarily associated with cytotoxic activity whereas CD4+ T helper (Th) cells are primarily associated with cytokine production. 
There are at least four phenotypes of effector Th cells, which can be characterised by their distinct effector functions and cytokine profiles ${ }^{1,14}$. DC production of IL-12 promotes Th1 differentiation ${ }^{15}$ and production of cytokines including IL-6 and TGF $\beta$ promote Th17 differentiation ${ }^{16,17}$. Th1 and Th17 cells, characterised by their production of IFN $\gamma$ and IL-17 respectively, mount responses against bacteria, protozoa, viruses and fungi ${ }^{1,18}$. Th1 cells activate innate cells such as $\mathrm{M} \varphi$ and are capable of inducing effector functions by CD8 cytotoxic T cells and B cells ${ }^{19}$. Th17 cells play an important role in resolving bacterial and fungal infections through recruitment and activation of neutrophils ${ }^{20}$. In contrast to Th1 and Th17 immunity, little is known about how DC induce Th2 responses in vivo ${ }^{21,22,23}$. Interestingly, environmental signals have been shown to induce expression of OX40L on DC which, upon interaction with $\mathrm{T}$ cell $\mathrm{OX} 40$, promotes Th2 differentiation. This suggests a role for receptor mediated interactions, in addition to soluble mediators, in Th2 polarisation ${ }^{24}$. Th2 cells produce IL-4, IL-5 and IL-13 and target multicellular pathogens such as helminths through recruitment and activation of mast cells, eosinophils and basophils ${ }^{25}$. Furthermore, Th2 immunity is strongly associated with allergic diseases and chemical mediators released by mast cells are thought to be responsible for causing allergic symptoms. In addition to effector Th cells, naïve CD4+ T cells can differentiate into Tregs, which are generated to maintain tolerance to self and innocuous antigens. There are two subtypes of Tregs: natural Tregs which develop in the thymus and inducible Tregs which develop from naïve Th cells in the periphery ${ }^{26}$. Inducible Tregs are commonly found at mucosal sites where immune cells are readily exposed to environmental antigen. Tregs produce antiinflammatory cytokines such as IL-10 and prevent inappropriate immune activation and assist with the resolution of inflammatory responses ${ }^{27}$. Importantly, Th cell subsets are not fixed and rigid but rather consist of a heterogeneous and plastic population that can alter their cytokine profile and may even produce multiple cytokines in response to environmental signals ${ }^{28}$. The extent of this plasticity depends on the differentiation state ${ }^{29,30}$ and phenotype of the T cell. For example, Th1 $17^{31,32}$ and Treg ${ }^{33,34}$ cells are capable of altering their cytokine profile throughout their differentiation process whereas Th1 and Th2 cells are thought to have a more stable phenotype and are less likely to change their cytokine profile once differentiation has been initiated ${ }^{30}$. Furthermore, recent evidence suggests there may be additional Th subsets that produce cytokines such as IL-9 ${ }^{35}$ and IL-22 ${ }^{36}$, although the roles these subsets play in immune responses are currently not well understood. 
Overall, DC provide a crucial link between innate and adaptive immunity and are responsible for initiating adaptive responses. Environmental signals received by DC and the subsequent signals between DC and T cells are important determinants of the type of immune response generated. Therefore, dysfunction in the signalling pathways leading to DC activation and signalling between DC and T cells can induce aberrant immune responses and subsequent pathology. Harnessing the power of the DC through targeted vaccines can set the stage for initiating appropriate immune responses.

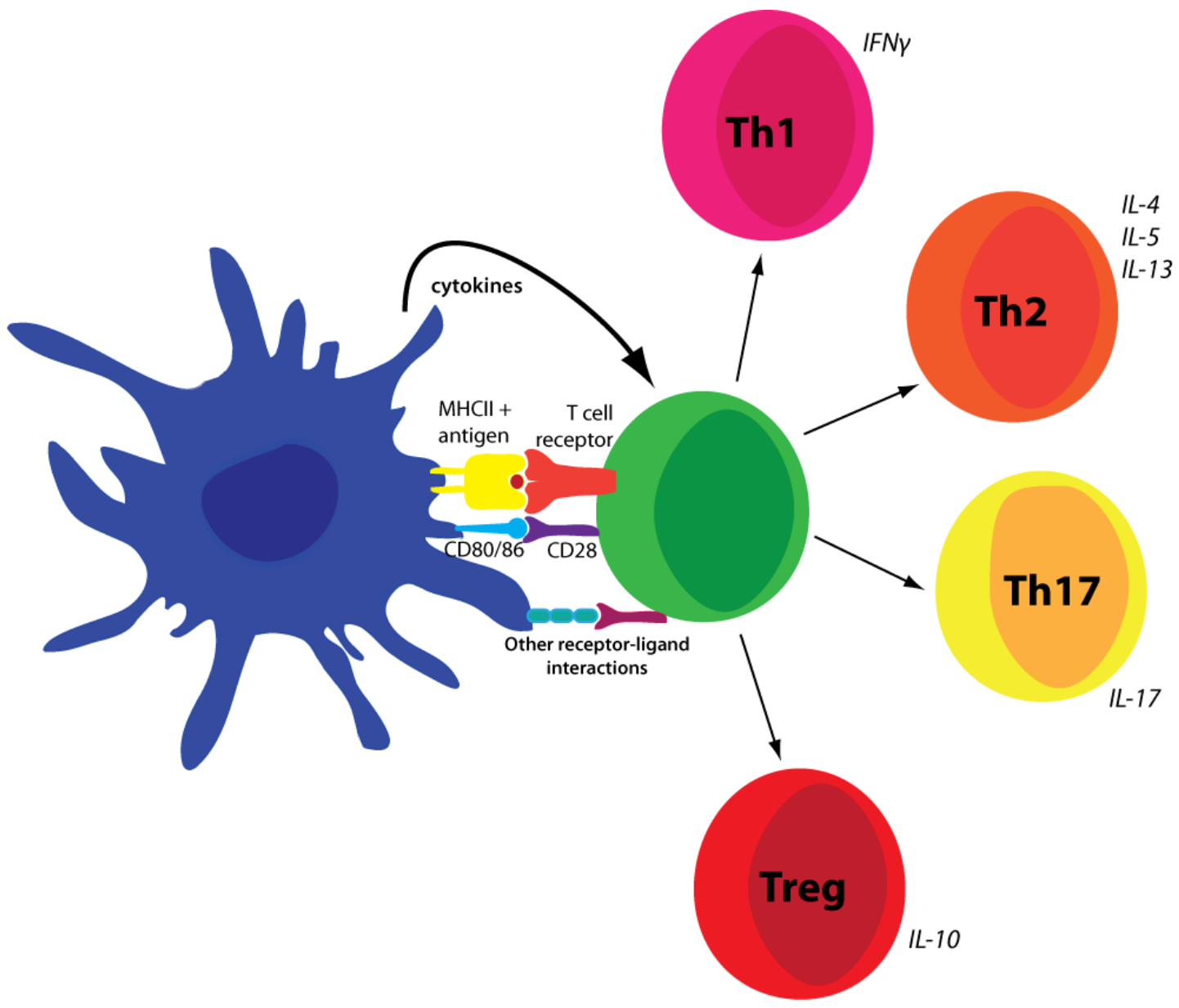

Figure 1.1: DC direct $T$ cell proliferation and differentiation

APC, such as DC, induce antigen specific $\mathrm{T}$ cell activation and expansion through presentation of antigen on MHC molecules and co-stimulation. Chemical mediators, such as cytokines, and additional receptor mediated interactions between the DC and T cell determine the effector cell phenotype the T cell will adopt. 


\subsubsection{Origin and function of DC populations}

In 1990, CD11c was recognised as a marker expressed solely on murine DC ${ }^{37}$. The accuracy of using CD11c to identify DC is currently being disputed ${ }^{38}$, with examples of CD11c expression overlapping with $\mathrm{M} \varphi$ populations in the spleen ${ }^{39}$, lung ${ }^{40}$, kidney ${ }^{41}$ and intestine ${ }^{42}$ and with CD8+ Tregs ${ }^{43}$. Nevertheless, CD11c remains a useful tool to identify DC in conjunction at with expression of MHCII molecules. There are at least three populations of DC that are developmentally distinct and specialise at recognising different classes of microbial agents due to their varying repertoires of PRRs ${ }^{10}$.

Conventional DC (cDC) originate from common myeloid progenitors from the bone marrow and can be further classified into peripheral tissue resident migratory DC or lymphoid tissue resident DC. At steady-state, migratory and lymphoid cDC can be identified by their differential expression of CD11c and MHCII. The migratory DC population express a CD11 ${ }^{\text {int }}$ MHCII $^{\text {hi }}$ phenotype while the lymphoid DC population express a CD11 $\mathrm{c}^{\text {hi }}$ MHCII $^{\text {int }}$ phenotype ${ }^{44}$. cDC can be divided into subsets by expression of additional cell surface markers including CD11b, CD103, CD4 and CD8 $\alpha$ and have differential abilities to prime CD4 and CD8 T cells ${ }^{45}$. Migratory DC are able to acquire antigen in the tissue, traffic to local lymph nodes and initiate T cell responses. In contrast, lymphoid DC remain in the lymphoid tissue and respond to local stimuli ${ }^{2}$.

Plasmacytoid DC (pDC) are found in peripheral tissues and lymphoid organs. They are identified by expression of B220, Ly6C, Siglec H and lower expression of CD11c compared to $\mathrm{cDC}{ }^{46,47}$. The best characterised function of $\mathrm{pDC}$ is to recognise viral motifs through TLR7 and TLR9. Following activation, pDC induce potent antiviral responses by initiating both innate and adaptive responses through production of type I interferons ${ }^{48}$. In addition, immature $\mathrm{pDC}$ have been implicated in driving tolerogenic responses in mice and humans through production of IL-10 ${ }^{27,46}$.

More recently, a subset of inflammatory monocyte-derived DC (moDC) has been identified. These cells are derived from monocytes circulating in the blood and differentiate into DC at sites of inflammation and are capable of antigen uptake and inducing $\mathrm{T}$ cell proliferation. 
MoDC are identified by their expression of the monocyte markers Ly6C and CD64 in conjunction with the DC markers CD11c and MHCII ${ }^{49}{ }^{50}$. MoDC have been shown to be key players in the initiation of adaptive responses in vaccination and tumour immunotherapy contexts ${ }^{50,51}$ and also in allergic airway disease ${ }^{49}$.

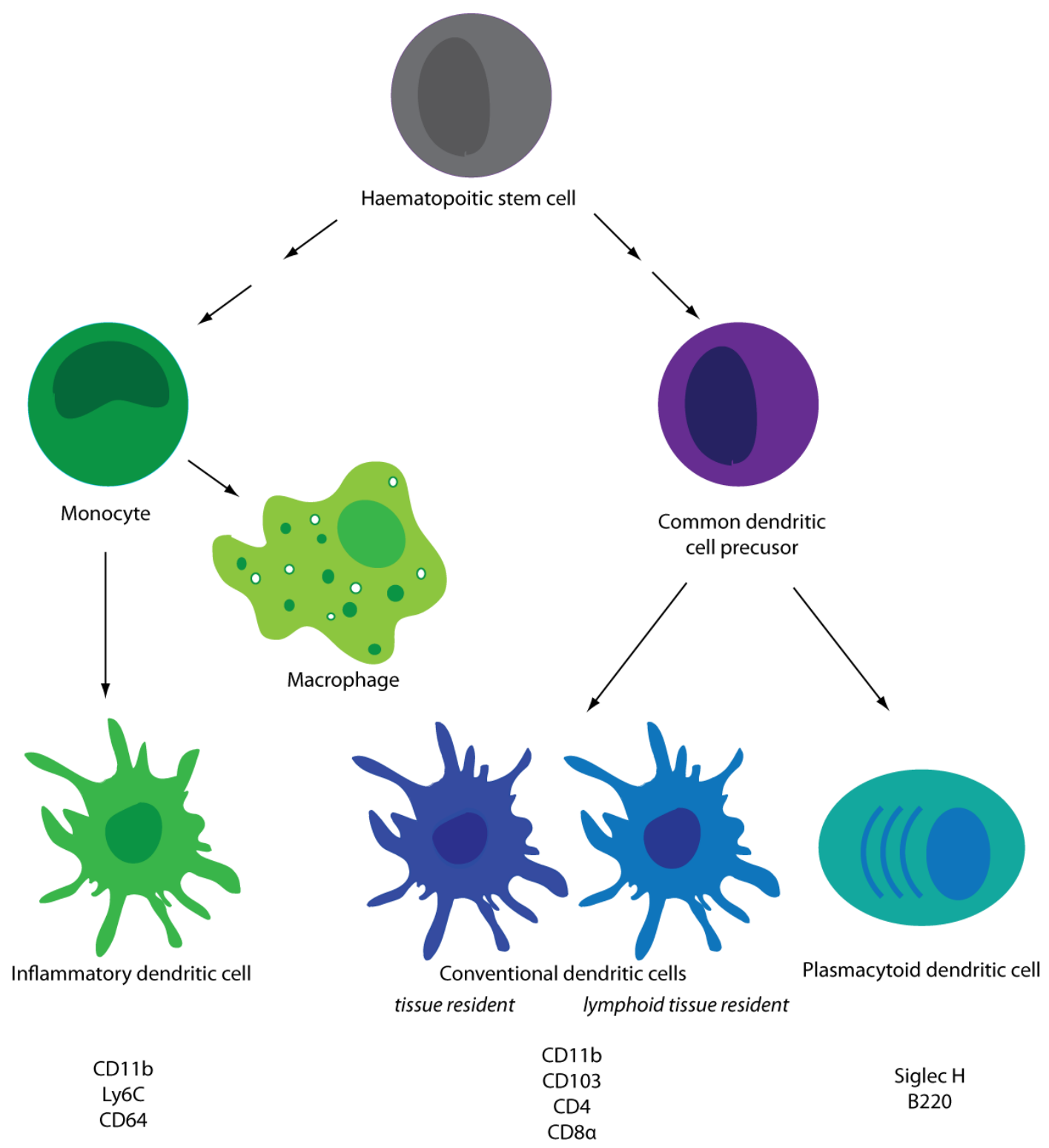

Figure 1.2: Lineages of DC 


\subsection{The intestinal immune system}

The immune system at mucosal sites, such as the intestine, requires tight regulation due to the constant exposure to foreign and microbial antigens from food proteins and the commensal microbiota. Development of effector responses to these antigens is associated with pathology such as food allergy and inflammatory bowel disease (see Section 1.2.3.). Therefore, the intestinal immune system is well adapted to develop tolerance to food proteins and commensal bacteria in order to prevent immune activation and maintain homeostasis. Importantly, despite the tolerogenic environment in the intestine, immune cells need to maintain the capacity to recognise and mount responses to pathogens. Highly specialised APC play key roles in regulating intestinal immune homeostasis by differentiating between innocuous antigens and pathogens (see Section 1.2.2.) and subsequently initiating appropriate responses. Therefore, a dichotomy exists in which recognition of antigen in the intestine can lead to either tolerance or immunity. Further understanding is required to determine whether environmental cues or activation of specific APC populations lead to the development of intestinal immunity.

\subsubsection{Intestinal immune homeostasis}

Intestinal immune homeostasis is maintained through a number of active processes which include oral tolerance ${ }^{52}$, production of secretory $\operatorname{IgA}$ by $\mathrm{B}$ cells ${ }^{53}$ and release of antimicrobial peptides and mucous by Paneth and goblet cells respectively ${ }^{54}$. Specialised populations of APC, unique to the intestine, are vital for promoting the development of oral tolerance $55,56,57,58$. Therefore, the primary focus of this section will be on oral tolerance.

Tolerance is a term used to describe immunological unresponsiveness, or lack of an effector response, to a specific antigen ${ }^{59}$. Oral tolerance involves two major components: the development of antigen specific Tregs ${ }^{60}$ and the development of anergic T cells ${ }^{61}$ to orally administered antigen. Firstly, Tregs play an important role in the suppression of inappropriate immune responses to self and innocuous antigens ${ }^{60}$. At mucosal sites, Tregs are responsible for creating a non-inflammatory environment by production of the cytokines IL-10 and TGF ${ }^{52}$. Exposure to antigens via the oral route induces antigen specific Tregs in 
the LP, Peyer's patches, MLN and at other peripheral sites ${ }^{52}$. Depletion of Tregs by targeting FoxP3 + cells results in a failure to develop tolerance to oral administration of ovalbumin antigen ${ }^{60}$. Secondly, oral tolerance can be achieved through $\mathrm{T}$ cell anergy (permanent non-responsiveness, usually due to activation with limited co-stimulation by CD80 and CD86 molecules) and deletion of food antigen specific T cells ${ }^{52,61}$. Interestingly, oral administration of antigen has been shown to result in tolerance not only locally but also systemically. For example, oral feeding of antigen prior to intraperitoneal immunisation with an adjuvant prevented a delayed hypersensitivity response upon antigen challenge in the ear pinna ${ }^{55}$.

In addition to $\mathrm{T}$ cell responses, intestinal immune homeostasis relies on physical defences that limit the interaction between luminal antigen and intestinal immune cells. A key component of this is the epithelial barrier which consists of a number of specialised cell types joined together by tight junctions ${ }^{54}$. A double layer of mucous on the apical side of the epithelial barrier limits the access of luminal antigens to epithelial and immune cells ${ }^{62}$ and acts as a scaffold for secretory $\operatorname{IgA}$ which further decreases the availability of antigen ${ }^{53}$. Finally, for protection against immune activation to commensal bacteria, Paneth cells (a constituent of the epithelial barrier) continually release antimicrobial peptides into the lumen 63.

Furthermore, accumulating evidence suggests that the intestinal microbiome has a critical role in educating the intestinal immune system to promote tolerance at steady-state ${ }^{64}$. Germfree mice are reared in a sterile environment and therefore are devoid of the natural microflora usually acquired at birth. These mice have been shown to spontaneously develop Th1 and Th17 cells ${ }^{65}$. Signalling through TLRs is a possible mechanism by which commensals and immune cells communicate ${ }^{66}$. This is supported by studies in which mice deficient in proteins involved in TLR signalling (MyD88 and Ticam1) had significantly lower number of Tregs upon colonisation compared to wildtype controls ${ }^{65}$. Thus, the composition of the microbiota is likely to be important in maintaining immune balance ${ }^{67}$. 


\subsubsection{Intestinal immunity}

While it is important that tolerance is maintained, the intestinal immune system needs to remain capable of inducing potent effector responses to eliminate pathogens. Due to the key role APC play in linking innate and adaptive immunity, it is likely environmental signals influence APC to drive either tolerogenic or effector responses. However, despite the wealth of recent research, it remains an enigma as to how intestinal APC are able to distinguish between innocuous antigens (such as food antigens and commensal microbes) and pathogens and subsequently mount the appropriate response.

Despite the fact that commensal microbes and pathogenic bacteria have the same PRR ligands, intestinal APC maintain tolerance to commensals but induce effector responses to pathogens. As mentioned above, one key component in maintaining intestinal homeostasis was the epithelial barrier which prevented the invasion of luminal proteins and commensal microbes into the LP. Therefore, it is possible that only pathogenic bacteria are able to gain entry into the LP and interact with immune cells which subsequently initiate effector responses. In support of this hypothesis, epithelial cells have been shown to differentially express TLRs on their apical and basolateral surfaces with one study showing some TLRs are restricted to the basolateral surface ${ }^{68}$. Several studies have shown TLR signalling is important for mobilisation and activation of intestinal APC in response to microbial stimuli 69, 70, 71, 72, 73 . However, other studies show non-invasive Salmonella is capable of recruiting but not maturing $\mathrm{DC}^{74}$. Furthermore, some populations of intestinal APC extend transepithelial dendrites into the lumen and directly sample antigen and thus will be continually in contact with foreign antigen ${ }^{71,75,76}$. Despite this, penetration by pathogenic strains through the epithelial barrier into the LP proves to be the best supported hypothesis on how effector responses are generated specifically towards pathogens in the intestine ${ }^{77}$.

In addition to bacterial invasion, it is also important to consider the complexity of the intestinal immune system and the likelihood that integration of signals from a number of cell types is required for the initiation of effector responses. Cross-talk between epithelial cells and APC has been implicated in regulating homeostasis ${ }^{78}$ so it stands to reason that signalling between these cell types may also be involved in the generation of effector responses. In support of this, TLR signalling in both epithelial cells and APC was required 
for mobilisation of APC following oral infection with Salmonella ${ }^{72}$. Furthermore, ATP, a recognised danger signal, induced epithelial cell production of pro-inflammatory cytokines and chemokines and recruited neutraphils ${ }^{79}$. In contrast, intestinal $\mathrm{M} \varphi$ failed to produce proinflammatory cytokines upon exposure to TLR ligands in vitro, suggesting that cross-talk between other immune cells and tolerogenic $\mathrm{M} \varphi$ may play a role in shifting the balance between immune activation and tolerance ${ }^{80,81}$. Thus, further study is required to understand how immunity is initiated towards pathogens but not food proteins and commensals in the intestine.

\subsubsection{Inappropriate intestinal immunity: food allergy and IBD}

Careful balance between tolerance and immunity is required to maintain homeostasis. When this balance becomes dysregulated inappropriate immune responses can develop to harmless antigens. Two common examples of this are food allergy and inflammatory bowel disease (IBD). Both of these conditions have experienced a sharp increase in prevalence over the last decade and represent a major health concern ${ }^{82,83,84,85,86}$.

\section{Food allergy}

Food allergy results from inappropriate immune activation of Th2 effector cells to specific food antigens. Th2 cells, characterised by their production of IL-4, IL-5 and IL-13, promote the production of antigen specific $\operatorname{IgE}$ antibodies which bind to the high affinity $\operatorname{IgE}$ receptor Fc£R1 on the surface of mast cells and basophils. Upon subsequent exposure, food antigens bind to IgE leading to cross-linking of FceR1 inducing mast cells and eosinophils release of inflammatory mediator molecules that are responsible for the symptoms associated with allergic disease ${ }^{87}$. It is these molecules, including serotonin and plateletactivating factor, that produce the symptoms of allergic diarrhoea and anaphylaxis in mouse models of food allergy ${ }^{88}$.

The processes that lead to allergic sensitisation and the initial development of Th2 cells are currently unknown and may occur at sites other than the intestine such as the skin ${ }^{89}$. Despite this, alterations in the microbiota composition are likely to be playing a role ${ }^{90}$. Perturbation in microbiota composition, specifically lack of species diversity, has been 
associated with the development of atopic dermatitis, allergic rhinitis and other allergic diseases in children 91, 92, 93. Germ-free mice have higher serum IgE titres and are more susceptible to models of food allergy compared to littermate controls further supporting the role for commensals in intestinal immune regulation ${ }^{94}$.

\section{Inflammatory bowel disease}

IBD is a class of pathologies characterised by chronic intestinal inflammation due to the induction of a strong Th1 response to commensal microbes ${ }^{95,96}$. Due to their roles in sampling and presentation of antigen, intestinal APC are thought to contribute to immune responses in instances of IBD ${ }^{97}$ with murine colitis studies showing the accumulation of activated APC throughout the LP and MLN ${ }^{98}$. Recent studies show these highly activated APC may be inflammatory DC derived from monocyte precursors that promote Th1 differentation ${ }^{99}$. However, it is not clear whether these activated APC are the primary drivers of inflammation or accumulate due to pro-inflammatory signals from other cell types.

In addition to intestinal APC, the epithelial barrier and microbial composition may be factors in IBD pathogenesis. At steady-state, the epithelial barrier and associated mucous layer play an important role in preventing sustained interaction between commensals and immune cells. IBD patients commonly have defects in the epithelial barrier which allows for commensals to move into the LP and interact with APC and other immune cells. Furthermore, bacterial dysbiosis is thought to contribute to disease pathogenesis as IBD patients show lower species diversity and other microbial imbalances compared to healthy controls ${ }^{66}$. Therefore, it is likely that alterations in microbial populations and their interactions with immune cells contribute to the development of these conditions.

\subsubsection{Intestinal APC hold the key to controlling aberrant immune responses}

Due to the central role APC play in linking innate and adaptive immunity, it is possible that APC mediate the balance between tolerance and immunity in the intestine. Therefore, understanding the composition of intestinal APC populations and the roles individual APC populations play in the control of intestinal immunity could provide vital insight into the

processes that lead to pathologies such as food allergy and IBD. This information could help 
in the development for therapies for food allergy and IBD by targeting specific cell surface molecules or APC subsets. 


\subsection{Intestinal APC}

Specialised subsets of APC are found throughout the intestinal lamina propria (LP) and associated lymphoid areas including the Peyer's patches, isolated lymphoid follicles and mesenteric lymph nodes (MLN) ${ }^{100}$. The intestinal APC population is highly heterogeneous consisting of both DC and $\mathrm{M} \varphi^{42,101}$. Furthermore, the composition of immune cells within each compartment is distinct ${ }^{102}$, adding an extra layer of complexity to the study of these populations. CD11c+ MHCII+ cells from the small intestinal LP are commonly divided into two populations, specifically, $\mathrm{CD} 103+\mathrm{DC}$ and $\mathrm{CX}_{3} \mathrm{CR} 1+\mathrm{M} \varphi{ }^{42,}{ }^{101}$. However, recent evidence suggests that these broad populations may contain distinct subsets ${ }^{103}$, and may not necessarily correspond to DC and $\mathrm{M} \varphi^{103}$.

\subsection{1. $\quad \mathrm{CD} 103+\mathrm{DC}$}

CD103+ DC from the small intestine can be subdivided into two populations based on their expression of CD11b and their requirements for development. CD103+ CD11b- DC are thought to have a similar phenotype and function to CD103+ CD11b- DC in other tissues and CD $8 \alpha+$ DC in lymphoid organs ${ }^{104}$. They arise from common DC precursors ${ }^{101,105}$ and rely on Flt3 $\mathrm{L}^{105}$ and the transcription factors $\mathrm{BatF} 3$ and interferon response element (IRF)-8 for their development ${ }^{104}$. In the intestine, CD103+ CD11b- cells are thought to predominantly reside in lymphoid organs such as Peyer's patches as mice lacking Peyer's patches were shown to have a substantial decrease in the number of CD103+ CD11b- DC but unaltered frequencies of other APC populations ${ }^{101,106}$. Small numbers of CD103+ CD11b- DC have been reported in LP, however, this appears to be dependent on the location within the intestine as CD103+ CD11b- DC have been found to be more abundant in distal parts of the small intestine and the colon ${ }^{102}$. In addition to CD103+ CD11b- DC, the LP contains a unique population of DC that co-express CD103 and CD11b. Like CD103+ CD11b- DC, CD103+ CD11b+ DC arise from common DC precursors but are dependent on GM-CSF ${ }^{105}$ and the transcription factors Notch2 ${ }^{103,107}$ and IRF4 ${ }^{108}$ for their development. Interestingly, Flt3L also appears to play a role in the development of CD103+ $\mathrm{CD} 11 \mathrm{~b}+\mathrm{DC}$ as mice lacking the receptor for Flt3L had reduced numbers of CD103+ CD11b+ DC present in the LP compared to wildtype controls ${ }^{101}$. Therefore, further 
investigation is needed to determine the full requirements for the development of steadystate LP DC. Confocal microscopy studies revealed that CD103+ CD11b+ DC preferentially localise deep within the LP to the centre of villi ${ }^{42,109}$ but can be recruited to the epithelial barrier during inflammation ${ }^{72}$.

Interestingly, there is a lack of evidence showing that CD103+ DC have the capacity to directly take up luminal antigen in vivo. Several recent studies suggest that LP DC may acquire antigen indirectly. One study proposed that CD103+ DC acquire antigen via gap junctions from the $\mathrm{CX}_{3} \mathrm{CR} 1+\mathrm{M} \varphi$ population located closer to the epithelium ${ }^{56}$, whereas others suggest that goblet cells transfer luminal antigen to DC ${ }^{110}$. Despite the lack of evidence for antigen uptake, CD103+ have been shown to have migratory capacities. Multiphoton confocal microscopy experiments using CD11c-eYFP and CX $\mathrm{CR}_{3}$-GFP mice elegantly demonstrate the presence of CD103+ DC in intestinal lymphatic vessels ${ }^{42}$. Furthermore, studies tracking the proliferation of APC using BrdU incorporation assays show that CD103+ and CD103- cells in the LP incorporate BrdU with faster kinetics than CD103+ cells in the MLN. This indicates that the BrdU was incorporated in the LP and the cell subsequently migrated to the MLN ${ }^{109}$. These studies highlighted the role for CD103+ DC in the transport of luminal antigen from the LP to the MLN. In fact, the CCR7 dependent migration of CD103+ DC ${ }^{101}$ has been shown to be vital for the generation of $\mathrm{T}$ cell responses to orally delivered antigen ${ }^{55,111}$.

Due to the migratory capacity of CD103+ DC, it is likely the CD103+ DC populations present in the MLN represent DC derived from the LP. Furthermore, CD103+ DC in the MLN were only found in the migratory $\mathrm{CD} 11 \mathrm{c}+\mathrm{MHCII}^{\mathrm{hi}}$ population ${ }^{103}$. Importantly, CD103+ DC from the MLN were capable of presenting orally delivered antigen ex vivo whereas CD103- DC populations were not ${ }^{109}$ suggesting that CD103+ DC in the transport and present luminal antigen in the draining lymph node.

\subsection{2. $\mathrm{CX}_{3} \mathrm{CR} 1+\mathrm{M} \varphi$}

$\mathrm{CX}_{3} \mathrm{CR} 1+$ cells originate from Ly6C $\mathrm{C}^{\text {hi }}$ blood monocytes ${ }^{105,}$,112, rely on M-CSF for their development ${ }^{101}$ and are continually replenished from the bone marrow ${ }^{113}$. Originally 
$\mathrm{CX}_{3} \mathrm{CR} 1+$ cells were classified as DC due to their expression of CD11c, however, evidence now supports $\mathrm{CX}_{3} \mathrm{CR} 1+$ cells are intestinal resident $\mathrm{M} \varphi$ as they have been shown to express $\mathrm{M} \varphi$ markers F4/80 ${ }^{114}$, CD64 ${ }^{113}$ and lack expression of DC specific protein zBTB46 ${ }^{115}$. Furthermore, microscopy studies have shown $\mathrm{CX}_{3} \mathrm{CR} 1+$ cells to have a vacuolar cytoplasm similar to that of $\mathrm{M} \varphi{ }^{101}$. For these reasons, I will be referring to $\mathrm{CX}_{3} \mathrm{CR} 1+$ cells as $\mathrm{M} \varphi$ throughout this thesis.

At steady-state, $\mathrm{CX}_{3} \mathrm{CR} 1+$ cells localise to the epithelium ${ }^{38,42,80}$. Experiments utilising $\mathrm{CX}_{3} \mathrm{CR} 1-\mathrm{GFP}$ mice nicely show the presence of transepithelial dendrites in the small intestine ${ }^{75}$ by which intestinal $\mathrm{M} \varphi$ sample antigen directly from the lumen ${ }^{71,75,76}$. Furthermore, feeding of fluorescently labelled antigen has been shown to accumulate within the $\mathrm{CX}_{3} \mathrm{CR} 1+$ population ${ }^{56,72}$. Interestingly, $\mathrm{CX}_{3} \mathrm{CR} 1+$ cells have not been detected in the afferent lymphatics or the MLN which suggests that they do not migrate to the lymph node ${ }^{42}$, although, there is current debate over whether $\mathrm{CX}_{3} \mathrm{CR} 1+$ cells may gain migratory capacity under inflammatory conditions ${ }^{116}$. Furthermore, $\mathrm{CX}_{3} \mathrm{CR} 1+$ cells are poor at inducing $\mathrm{T}$ cell proliferation ${ }^{42,101}$. Together, these findings suggest that following acquisition of antigen, $\mathrm{CX}_{3} \mathrm{CR} 1+\mathrm{M} \varphi$ pass antigen onto a migratory DC population. The process by which this occurs is not clear, although as discussed above, a recent study demonstrates the role of gap junctions in the transfer of antigen between $\mathrm{CX}_{3} \mathrm{CR} 1+$ and $\mathrm{CD} 103+$ populations. The study found that MLN DC from mice deficient in Connexin 43, a protein central to gap junction formation, were unable to drive $\mathrm{T}$ cell proliferation to orally delivered antigen ${ }^{56}$. This implies that gap junctions are critical for antigen trafficking from the LP to the MLN.

\subsubsection{Other APC populations}

A small population of $\mathrm{CX}_{3} \mathrm{CR} 1^{\text {int }}$ cells has been reported in the LP at steady-state. Similar to $\mathrm{CX}_{3} \mathrm{CR} 1^{\text {hi }} \mathrm{M} \varphi, \mathrm{CX}_{3} \mathrm{CR} 1^{\text {int }}$ cells have been shown to originate from Ly6C $\mathrm{C}^{\text {hi }}$ blood monocytes with adoptively transferred Ly6C $\mathrm{C}^{\text {hi }}$ monocytes differentiating into $\mathrm{CD} 11 \mathrm{c}+\mathrm{MHCII}+$ $\mathrm{CX}_{3} \mathrm{CR} 1^{\text {int }} \mathrm{Ly} 6 \mathrm{C}^{\text {lo }}$ cells ${ }^{99}$. However, unlike $\mathrm{CX}_{3} \mathrm{CR} 1^{\text {hi }}$ cells, $\mathrm{CX}_{3} \mathrm{CR} 1^{\text {int }}$ cells expand in response to Flt3L ${ }^{42}$, express lymph node homing receptor CCR7 ${ }^{99}$, have been found in the intestinal lymph and have some capacity for promoting $\mathrm{T}$ cell proliferation ${ }^{42,} 106$ suggesting they have functional characteristics of DC. Interestingly, during intestinal inflammation, the 
frequency of $\mathrm{CX}_{3} \mathrm{CR} 1^{\text {int }}$ cells in the LP increases significantly ${ }^{99,}{ }^{117}$ and may promote the migration of this population ${ }^{116}$. Therefore, it is possible that blood monocytes preferentially differentiate into $\mathrm{CX}_{3} \mathrm{CR} 1^{\text {int }}$ cells or $\mathrm{CX}_{3} \mathrm{CR} 1^{\text {hi }} \mathrm{M} \varphi$ depending on environmental signals.
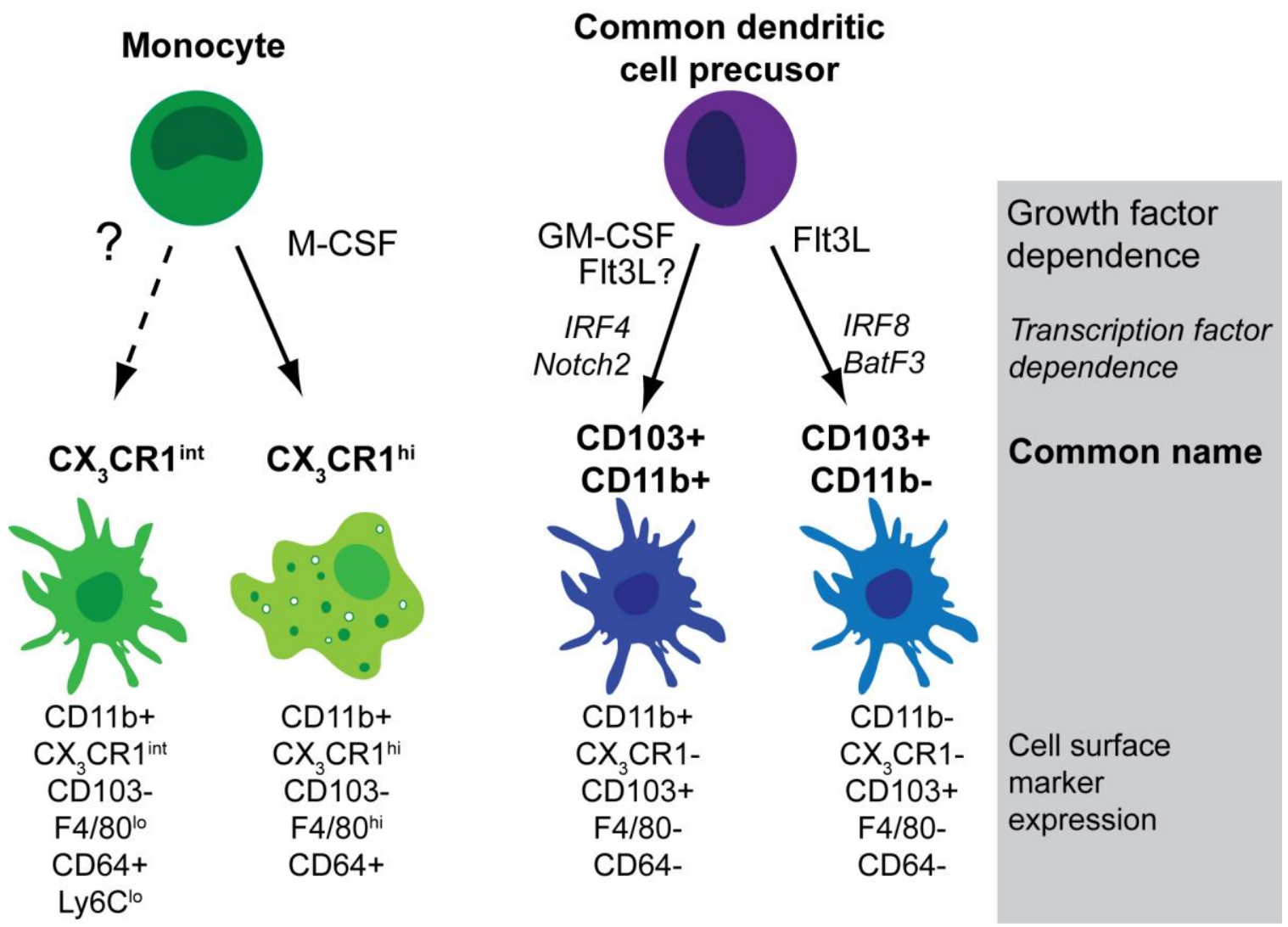

Figure 1.3: Lineage of intestinal APC 
Table 1: Summary of cell surface markers expressed by intestinal APC populations

\begin{tabular}{|c|c|c|c|}
\hline Marker & $\begin{array}{c}\text { Also known } \\
\text { as... }\end{array}$ & $\begin{array}{c}\text { Expressed } \\
\text { by... }\end{array}$ & Function \\
\hline CD11b & $\begin{array}{l}\text { Integrin } \alpha \mathrm{M} \text {, } \\
\text { CR3 }\end{array}$ & $\begin{array}{l}\text { Intestinal DC } \\
\text { and } \mathrm{M} \varphi\end{array}$ & Adhesion molecule \\
\hline CD11c & Integrin $\alpha \mathrm{X}$ & $\begin{array}{l}\text { Intestinal DC } \\
\text { and } \mathrm{M} \varphi\end{array}$ & $\begin{array}{l}\text { Adhesion molecule commonly used to } \\
\text { identify DC populations. However, is also } \\
\text { expressed by intestinal } \mathrm{M} \varphi{ }^{42} \text {. }\end{array}$ \\
\hline CD64 & Fc $\gamma R$ 1 & $\begin{array}{l}\text { Monocyte- } \\
\text { derived } \\
\text { intestinal APC: } \\
\text { M } \varphi \text { (moDC?) }\end{array}$ & $\begin{array}{l}\text { High affinity IgG receptor, although, it is } \\
\text { unclear how binding of IgG to this } \\
\text { receptor influences immunity }{ }^{118} \text {. CD } 64 \\
\text { clearly distinguishes } \mathrm{M} \varphi \text { from DC in the } \\
\text { intestine at steady-state }{ }^{113} \text {. }\end{array}$ \\
\hline CD103 & Integrin $\alpha \mathrm{E}$ & Intestinal DC & Adhesion molecule \\
\hline $\mathrm{CX}_{3} \mathrm{CR} 1$ & $\begin{array}{l}\text { Fractalkine } \\
\text { receptor }\end{array}$ & $\begin{array}{l}\text { Intestinal M } \varphi \\
\text { (moDC?) }\end{array}$ & $\begin{array}{l}\text { Chemokine receptor: regulates IL-10 } \\
\text { production and formation of transepithelial } \\
\text { dendrites by intestinal } \mathrm{M} \varphi{ }^{60} \text {. }\end{array}$ \\
\hline F4/80 & $\begin{array}{l}\text { EGF-like } \\
\text { module } \\
\text { containing, } \\
\text { mucin-like } \\
\text { hormone } \\
\text { receptor }\end{array}$ & Intestinal $\mathrm{M} \varphi$ & $\begin{array}{l}\text { Highly expressed by mature tissue-resident } \\
\text { M }{ }^{119,120} \text { such as those found in the } \\
\text { intestine. Signalling though F4/ } 80 \text { has been } \\
\text { associated with the induction of Tregs in } \\
\text { the periphery }{ }^{121} \text {. }\end{array}$ \\
\hline Ly6C & - & $\begin{array}{l}\text { Monocyte- } \\
\text { derived } \\
\text { intestinal APC: } \\
\text { M } \varphi \text { (moDC?) }\end{array}$ & $\begin{array}{l}\text { Ly6C on CD8+ T cells is associated with } \\
\text { regulating T cell proliferation }{ }^{122} \text {, cytolytic } \\
\text { activity }{ }^{123} \text { and lymph node homing }{ }^{124} \text {. The } \\
\text { function of Ly6C on other immune cell } \\
\text { types is not clear and requires further } \\
\text { investigation. }\end{array}$ \\
\hline
\end{tabular}




\subsection{APC in the regulation of intestinal immune responses}

\subsubsection{Intestinal APC and the promotion of tolerance}

Migration of antigen-loaded intestinal APC to the MLN is essential for the induction of tolerance to the vast amount of foreign antigen ${ }^{55}$. This is supported by experiments which show that in mice where DC are unable to migration to the MLN ${ }^{111}$ or acquire antigen ${ }^{56}$, were unable to develop tolerance to orally delivered antigen. Interestingly, both CD103+ CD11b- and CD103+ CD11b + DC appear to play a role in promoting tolerance as ablation of both of these populations was required to decrease the number of Tregs in the MLN ${ }^{81}$. In order to maintain tolerance, CD103+ DC are required to simultaneously promote the development of Tregs and inhibit the development of effector T cells in the MLN ${ }^{60,125}$. Differentiation of naïve $\mathrm{T}$ cells to Tregs is promoted by CD103+ DC production of chemical mediators such as retinoic acid (RA) and TGF- $\beta$ 57, 58, 126. CD103+ DC are specifically equipped with aldehyde dehydrogenase enzymes, including retinal dehydrogenase (RALDH), which are required for the conversion of dietary vitamin A derivatives to RA. In addition, CD103+ DC have been shown to produce thymic stromal lymphopoietin (TSLP), an epithelial associated cytokine that has been shown to promote the induction of Th2 responses ${ }^{24}$. However, in the intestine, DC derived TSLP may play a role in promoting Treg development and inhibiting the development of Th17 cells ${ }^{127}$.

In addition to promoting Treg differentiation, RA is required for promoting the upregulation of gut-homing receptor $\alpha 4 \beta 7$ and gut chemokine receptors CCR9 and CCR10 on T cells allowing for them to traffic to the LP and commence their suppressor activities ${ }^{70,72,73}$. Interestingly, mice deficient in components of gut homing molecules or gut homing receptor MAdCAM-1 were unable to establish oral tolerance suggesting that gut homing imprinted by intestinal DC may also play a role in promoting tolerance ${ }^{60}$. Furthermore, intestinal DC play an important role in gut imprinting $\mathrm{B}$ cells which are required to produce neutralising secretory $\operatorname{Ig} \mathrm{A}^{128,129}$.

$\mathrm{CX}_{3} \mathrm{CR} 1+\mathrm{M} \varphi$ also play an important role promoting tolerance at steady-state. Primarily, this

is through production of the anti-inflammatory cytokine IL-10 which helps with the 
maintenance of FoxP3 expression and suppressive activities by Tregs in the $\mathrm{LP}^{80,130}$. Binding of fractalkine $\left(\mathrm{CX}_{3} \mathrm{CL} 1\right)$ to its receptor $\mathrm{CX}_{3} \mathrm{CR} 1$ is thought to be important for promoting production of specific cytokines ${ }^{131}$. Therefore, it is interesting to note that mice deficient in $\mathrm{CX}_{3} \mathrm{CR} 1$ have a significantly lower frequency of IL-10+ intestinal $\mathrm{M} \varphi$ and subsequently have reduced numbers of Tregs in the LP compared to wild-type controls ${ }^{60}$. Furthermore, $\mathrm{CX}_{3} \mathrm{CR} 1$ expression was found to be important for establishing oral tolerance as sensitised $\mathrm{CX}_{3} \mathrm{CR} 1$ deficient mice failed to suppress delayed-type hypersensitivity responses following oral delivery of antigen ${ }^{60}$.

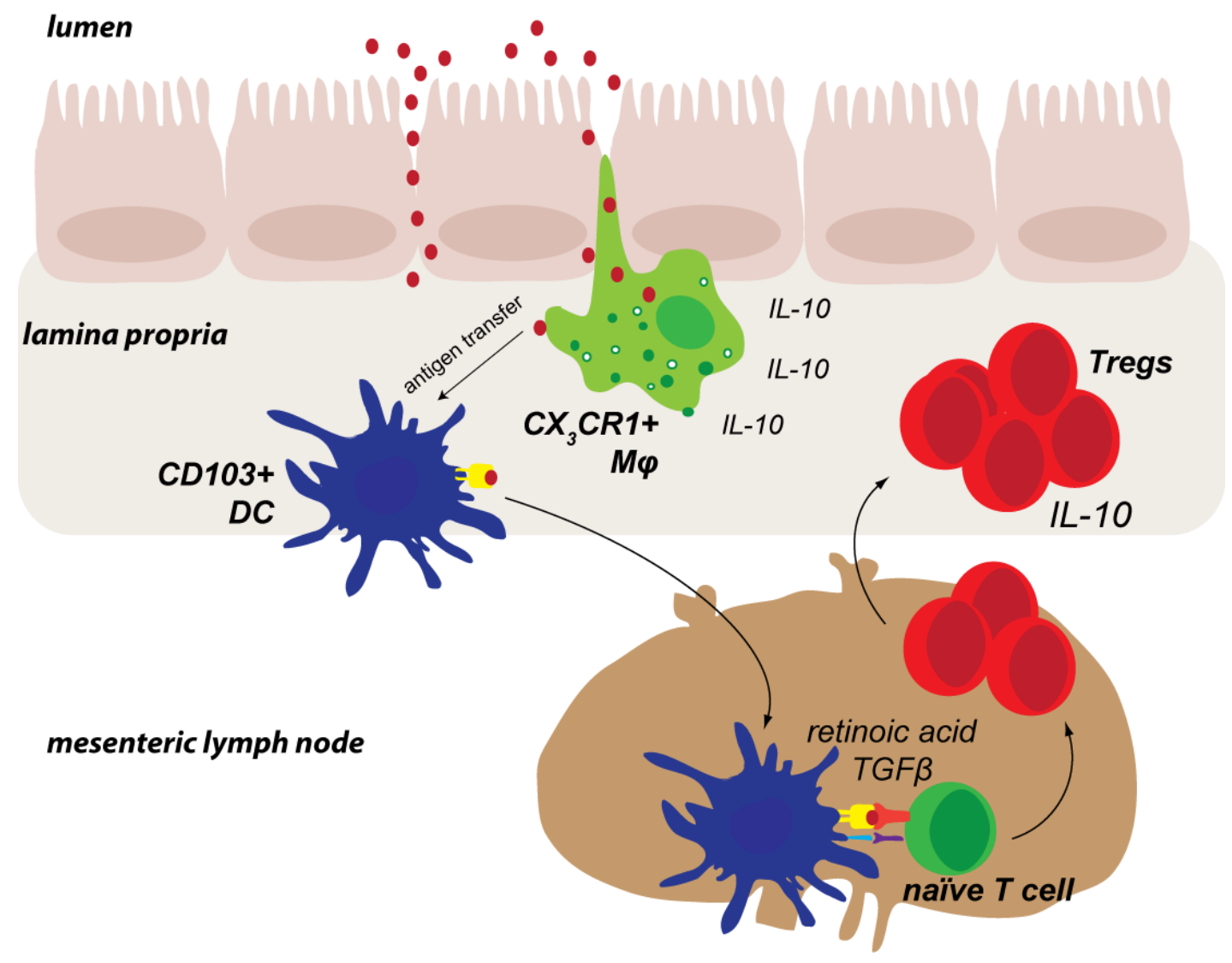

Figure 1.4: Intestinal APC regulate oral tolerance 


\subsubsection{APC and intestinal immunity}

The role of intestinal APC in the promotion of tolerance is relatively well understood. However, the mechanisms by which intestinal APC identify threats and initiate effector T cell responses is less well defined. Furthermore, it is unclear whether specific subsets within characterised APC populations are responsible for driving different immune responses or whether environmental signals condition APC to drive appropriate effector T cell responses.

Th1 responses are characteristic of bacterial, protozoan and viral infections ${ }^{73,108,132,133}$ but have also been implicated in IBD pathology ${ }^{95}$. A subset of intestinal CD103+ CD11b$\mathrm{CD} 8 \alpha+\mathrm{DC}$ have been implicated in promoting Th1 responses in response to TLR stimulation. This subset was found to produce IL- 6 and IL-12 and drive IFN $\gamma$ but not IL-17 production by antigen specific CD4 T cells in vitro and in vivo when exposed to TLR agonists 73. Similar experiments showed that CD103+ CD11b+ DC also drove Th1 differentiation in response to TLR ligands ${ }^{133}$. Furthermore, a subset of $\mathrm{CX}_{3} \mathrm{CR} 1^{\text {int }}$ cells has been associated with inflammatory Th1 responses in mouse models of colitis. Studies have shown during colonic inflammation there is a transient increase in $\mathrm{CX}_{3} \mathrm{CR} 1^{\text {int }}$ cells that have a proinflammatory transcriptomic signature and produce large amounts of pro-inflammatory cytokines 117, 134, 135. Ablation of the $\mathrm{CX}_{3} \mathrm{CR} 1^{\text {int }}$ subset significantly reduced colonic inflammation, further implicating this subset in driving effector $\mathrm{T}$ cell responses ${ }^{99}$. The roles of intestinal monocytes and $\mathrm{M} \varphi$ have also been assessed and were found to provide cytokine help to established Th1 immune responses but were not required for $\mathrm{T}$ cell priming. Furthermore, monocytes and $\mathrm{M} \varphi$ from the small intestine produce IL-12 in instances of inflammation and thus, contribute to T cell IFN $\gamma$ responses ${ }^{136}$.

In addition to driving Th1 responses, CD103+ CD11b+ DC have been specifically implicated in driving Th17 ${ }^{80,81,102,133,137}$ and IL-22/IL-23 ${ }^{63}$ effector responses in the intestine. Studies have shown CD103+ CD11b+ DC promote Th17 differentiation without stimulation in vitro, however, Th17 differentiation was enhanced following stimulation with a TLR5 agonist ${ }^{80,102,133}$. The involvement of CD103+ CD11b+ DC in Th17 cell homeostasis was further supported by experiments showing that conditional depletion of CD103+ CD11b+ DC resulted in a significant decrease in Th17 cells in the MLN ${ }^{81}$. Moreover, specific deletion of Notch2 in CD11c+ cells has been shown to effectively reduce the 
numbers of CD103+ CD11b+ DC in the intestine without affecting other APC populations. These mice succumbed to infection with the bacteria Citrobacter rodentium whereas BatF3 knockout mice, deficient in CD103 + CD11b- DC, and wildtype mice effectively cleared infection. The inability for Notch2 deficient DC to promote infection clearance was ascribed to their inability produce the cytokine IL-23 ${ }^{103}$ which is important for promoting antimicrobial activities by other immune cells and epithelial cells (see Section 1.2.2) ${ }^{138}$.

The specific requirements to drive Th2 responses in the intestine and other tissues have been the focus of much study and review 1, 139, 140, 141, 142. However, the APC subsets and mechanisms involved in the promotion Th2 immunity are not known. Studies in helminth infection models have reported changes in MLN DC composition, notably, an increase in $\mathrm{CD} 11 \mathrm{c}^{\text {lo }} \mathrm{CD} 103-\mathrm{DC}{ }^{143}$, although the relevance of this finding is unclear. Current evidence suggests environmental signals may alter expression of cell surface markers on APC that promote Th2 differentiation ${ }^{24}$. In the intestine, expression of a number of "Th2 skewing" markers, including OX40 ligand (OX40L) ${ }^{86}$ and T cell immunoglobulin and mucin domain containing-protein (TIM)-4 ${ }^{144}$, on CD103+ DC has been associated with the development of Th2 immunity in mouse models of food allergy. TIM-4 is predominantly expressed on $\mathrm{M} \varphi$ populations ${ }^{145,146}$ but has been shown to be upregulated on DC stimulated with TLR ligands ${ }^{147,148}$. DC expressing TIM-4 were found to promote Th2 differentiation in vitro and in vivo ${ }^{144,147,149}$ and blockade of TIM-4 or, one of its target receptors, TIM-1 prevented Th2 differentiation and allergic anaphylaxis thus implicating Th2 promotion via signalling through the TIM-4/TIM-1 interaction ${ }^{144}$. 


\subsection{Cholera toxin: a potent mucosal adjuvant}

Cholera toxin (CT) is a potent mucosal adjuvant that is produced by the bacterium Vibrio cholera and consists of two subunits, a monomeric A subunit and a pentameric B subunit. CT is known to exert its pathogenic effects on intestinal epithelial cells through binding of CT-B to cell surface GM-1 ganglioside receptors which facilitates entry of CT-A into the cell. Once inside the cell, CT-A causes the continual activation of adenylate cyclase by influencing Gprotein activation which results in efflux of water and electrolytes and ultimately, global cellular dysregulation ${ }^{150,151,152,153}$.

In addition to its pathological effects on epithelial cells, CT is also known to promote effector $\mathrm{T}$ cell responses to co-delivered antigen and thus is commonly used as an adjuvant at mucosal sites. Interestingly, CT has been reported to preferentially induce Th2 responses 150, 154, 155, 156, although, others claim CT has general stimulatory effects on immune cells driving Th1 and Th2 responses simultaneously ${ }^{151,157,158,159}$. To induce its immunostimulatory effects, CT primarily affects the phenotype and function of APC 86, 144, 153, 158, 159, 160, 161 . Experiments using bone marrow chimeric mice deficient in GM1 expression on haematopoietic cells, show that direct interaction between CT and DC was required for adjuvant activity. This was further confirmed using CD11c-DTR GM1-/- mixed bone marrow chimeric mice. DT depletion in these mice failed to induce antigen specific T cell proliferation following an oral dose of antigen with $\mathrm{CT}{ }^{160}$. In addition, CT has been investigated as a potential vaccine adjuvant. Co-administration of CT with antigen resulted in dermal DC activation, migration and induction of potent CD8 T cell responses ${ }^{159}$.

The ability of CT to induce potent $\mathrm{T}$ cell responses to co-delivered antigen and promote epithelial cell dysfunction allowing for increase antigen availability, makes it a useful tool to study the effect of an immunostimulatory agent on intestinal immune cells. Due to its Th2 skewing properties, CT is already commonly used to sensitise mice to orally co-delivered antigen in models of food allergy ${ }^{162}$. Therefore, I used oral CT treatment to study the phenotype and functions of intestinal APC subsets in an environment in which effector T cell responses were initiated. 


\subsection{Rationale and hypothesis}

APC are the gatekeepers to adaptive immunity. They have the capacity to acquire antigen and direct the appropriate $\mathrm{T}$ cell responses. Due to the high antigenic load at mucosal sites, intestinal APC are specialised to induce tolerance at steady-state but remain capable of inducing potent immune responses to pathogens. Inappropriate immune activation is associated with a number of pathologies including food allergy and inflammatory bowel disease. The mechanisms by which APC drive intestinal immune responses in infection and pathology is currently unknown. I used the mucosal adjuvant CT to induce an environment in which effector immune responses were initiated in order to study intestinal APC. As signals from APC direct $\mathrm{T}$ cell proliferation and differentiation, I hypothesised that $\mathrm{CT}$ treatment would alter the phenotype and function of some APC in the LP and MLN and that these APC would be responsible for the generation of effector T cell responses to CT. 


\subsection{Aims of this thesis}

In this chapter I have highlighted the key roles APC have in the initiation and direction of adaptive immune responses. Regulation of adaptive immune responses is particularly important in the intestine where APC are constantly exposed to microbial agents and foreign antigen. At steady-state intestinal APC drive tolerance through the promotion of Treg differentiation and production of the anti-inflammatory cytokine IL-10. However, intestinal APC retain the capacity to induce appropriate immune responses to pathogens or inappropriate immune responses to food proteins and commensal bacteria. The mechanisms that determine APC promotion of either Tregs or effector T cell differentiation remain elusive. Oral administration of the mucosal adjuvant CT can provide a useful setting to study APC in an immunostimulatory environment. Therefore, the overall aim of this study was to compare APC subset composition, phenotype and function between steady-state and following oral administration of CT.

Specifically my aims were:

1. To assess the APC subset composition and phenotype from the LP and MLN at steady-state.

2. To identify whether CT treatment alters LP and MLN APC subset composition and phenotype.

3. To assess and compare functional characteristics of APC subsets between steadystate and CT treatment.

4. To characterise $\mathrm{T}$ cell expansion and cytokine production following oral administration of CT. 


\section{Chapter 2: Materials and Methods}




\subsection{Materials}

\subsubsection{Labware}

\begin{tabular}{|c|c|}
\hline Product & Supplier \\
\hline $\begin{array}{l}\text { Acrodisc }{ }^{\circledR} 32 \mathrm{~mm} \text { syringe filter with } 0.2 \mu \mathrm{m} \text { Supor }{ }^{\circledR} \\
\text { membrane }\end{array}$ & PALL Life Sciences (Cornwall, UK) \\
\hline Axygen ${ }^{\circledR}$ sterile pipet tips $1000,200 \& 10 \mu \mathrm{L}$ & $\begin{array}{l}\text { Axygen Scientific Inc. (Union City, CA, } \\
\text { USA) }\end{array}$ \\
\hline Falcon $^{\mathrm{TM}} 1,3,5,10 \mathrm{~mL}$ Tuberculin syringes & \multirow{8}{*}{$\begin{array}{l}\text { Becton Dickinson (BD) Biosciences } \\
\text { (Bedford, MA, USA) }\end{array}$} \\
\hline $\begin{array}{l}\text { Falcon }^{\mathrm{TM}} \text { polystyrene sterile conical tubes: Blue Max } \\
50 \mathrm{~mL} \text { and Blue Max Jr. } 15 \mathrm{~mL}\end{array}$ & \\
\hline $\begin{array}{l}\text { Falcon }^{\mathrm{TM}} \text { polystyrene sterile multiwall tissue culture } \\
\text { plates: } 6 \text { well, } 12 \text { well, } 24 \text { well, } 48 \text { well and } \\
\text { MicrotestTM Ubottom } 96 \text { well plates }\end{array}$ & \\
\hline $\begin{array}{l}\text { Falcon }^{\mathrm{TM}} 2,5,10 \text { and } 25 \mathrm{~mL} \text { Polystyrene serological } \\
\text { pipettes }\end{array}$ & \\
\hline Falcon ${ }^{\mathrm{TM}}$ Nylon cell strainers: $40 \mu \mathrm{m}$ and $70 \mu \mathrm{m}$ & \\
\hline Falcon $^{\mathrm{TM}} 5 \mathrm{~mL}$ polystyrene round bottom tubes & \\
\hline PrecisionGlideTM needles: 18, 25 gauge $(\mathrm{G})$ & \\
\hline $\begin{array}{l}\text { Ultra-FineTM needle insulin syringes: } 29 \text { gauge } 0.3 \text {, } \\
0.5 \& 1 \mathrm{~mL}\end{array}$ & \\
\hline Disposable Biopsy Punch (3mm diameter) & Kai Industries (Seki City, Japan) \\
\hline Gavage needles: 22G, 1.5 ” stainless steel & Cadence Science (Staunton, VA, USA) \\
\hline MACS $\AA$ AutoMACS separation columns & \multirow{2}{*}{$\begin{array}{lccc}\text { Miltenyi } & \text { Biotech } & \text { GmbH } & \text { (Bergisch } \\
\text { Gladbach, Germany) } & & \end{array}$} \\
\hline MACS® pre-separation filters, $30 \mu \mathrm{m}$ & \\
\hline Carbon Steel Surgical Blades number $10 \& 22$ (sterile) & $\begin{array}{l}\text { Swann-Morton }{ }^{\circledR} \quad \text { Limited } \\
\text { England) }\end{array}$ \\
\hline Titertube ${ }^{\circledR}$ Micro Tubes & Bio-Rad (Hercules, CA, USA) \\
\hline Nunc-Immuno $^{\text {TM }}$ MicroWell $^{\text {TM }} 96$ well solid plates & Sigma-Aldrich (St. Louis, MO, USA) \\
\hline
\end{tabular}




\subsubsection{Reagents}

\begin{tabular}{|c|c|}
\hline Product & Supplier \\
\hline 2-mercapto-ethanol (2-ME) $55 \mathrm{mM}$ solution & Sigma-Aldrich (St. Louis, MO, USA) \\
\hline $\begin{array}{l}\text { ALDEFLUOR }^{\mathrm{TM}} \text { kit (Aldehyde Dehygrogenase - } \\
\text { based cell detection kit) }\end{array}$ & $\begin{array}{l}\text { Stem Cell Technologies } \quad \text { Victoria, } \\
\text { Australia) }\end{array}$ \\
\hline Ammonium chloride powder & \multirow[t]{2}{*}{ Sigma-Aldrich (St. Louis, MO, USA) } \\
\hline Ampicillin & \\
\hline Annexin-V Apoptosis Detection Kit II & BD Pharmingen (San Diego, CA, USA) \\
\hline Bovine serum albumin (BSA), lyophilised powder & ICPbio Ltd. (Auckland, NZ) \\
\hline Brefeldin A solution & eBioscience (San Diego, CA, USA) \\
\hline Cholera toxin, lyophilised powder & Sigma-Aldrich (St. Louis, MO, USA) \\
\hline Cytofix/Cytoperm kit & BD Pharmingen (San Diego, CA, USA) \\
\hline Dexamethasone - water soluble & Sigma-Aldrich (St. Louis, MO, USA) \\
\hline Dextran sulfate sodium (DSS) salt & MP Biomedicals (Solon, OH, USA) \\
\hline DNase I powder & Roche (Mannheim, Germany) \\
\hline Dynabeads ${ }^{\circledR}$, FlowComp ${ }^{\text {TM }}$ Mouse CD4 & Invitrogen (Auckland, New Zealand) \\
\hline Ethanol, $100 \%$ & Carlo Erba Reagents (Milan, Italy) \\
\hline $\begin{array}{l}\text { Ethylenediaminetetraacetic acid (EDTA) } \\
\text { powder }\end{array}$ & Sigma-Aldrich (St. Louis, MO, USA) \\
\hline Fetal calf serum (FCS) & Invitrogen (Auckland, New Zealand) \\
\hline Gentamicin sulfate & \multirow[t]{2}{*}{ Sigma-Aldrich (St. Louis, MO, USA) } \\
\hline Goat serum & \\
\hline GolgiStop & BD Pharmingen (San Diego, CA, USA) \\
\hline $\begin{array}{l}\text { Hank's Balanced Salt Solution (HBSS) supplemented } \\
\text { with calcium chloride and magnesium chloride }\end{array}$ & Invitrogen (Auckland, New Zealand) \\
\hline Hepes buffer $1 \mathrm{M}$ solution & Sigma-Aldrich (St. Louis, MO, USA) \\
\hline $\begin{array}{l}\text { Iscove's Modified Dulbecco Medium } \\
\text { (IMDM) supplemented with GlutaMAX TM, } \\
25 \mathrm{mM} \text { HEPES buffer and } 3.024 \mathrm{mg} / \mathrm{L} \\
\mathrm{NaHCO}_{3}\end{array}$ & Invitrogen (Auckland, New Zealand) \\
\hline
\end{tabular}




\begin{tabular}{|c|c|}
\hline Liberase TL powder & Roche (Mannheim, Germany) \\
\hline Interleukin (IL)-2 & $\begin{array}{l}\text { IL-2 producing IL2L6 cell line, generated } \\
\text { by the modification of murine J558 } \\
\text { parental line }\end{array}$ \\
\hline $\begin{array}{l}\text { Magnetic separation (MACS) beads, anti-CD11c } \\
\text { microbeads }\end{array}$ & $\begin{array}{l}\text { Miltenyi Biotech GmbH } \quad \text { (Bergisch } \\
\text { Gladbach, Germany) }\end{array}$ \\
\hline Metronidazole & Sigma-Aldrich (St. Louis, MO, USA) \\
\hline Neomycin trisulfate salt hydrate & Sigma-Aldrich (St. Louis, MO, USA) \\
\hline $\begin{array}{l}\text { Neurotactin (NTN), CX }{ }_{3} \text { CL1- human Fc fusion } \\
\text { protein }\end{array}$ & $\begin{array}{l}\text { Kindly provided by Prof. Steffen Jung } \\
\text { (Weizmann Institute of Science, Rehovot, } \\
\text { Israel) }\end{array}$ \\
\hline Ovalbumin (OVA) grade $\mathrm{V}$ protein powder & Sigma-Aldrich (St. Louis, MO, USA) \\
\hline $\begin{array}{l}\text { OVA }_{323-339} \text { (ISQAVHAAHAEINEAGR) } \\
\text { Peptide (ISQ) }\end{array}$ & Mimotopes (Victoria, Australia) \\
\hline OVA conjugated to AlexaFluor-647 & $\begin{array}{l}\text { Molecular Probes }{ }^{\circledR} \quad \text { (Eugene, Oregon, } \\
\text { USA) }\end{array}$ \\
\hline Penicillin-streptomycin & Invitrogen (Auckland, New Zealand) \\
\hline Phosphate buffer saline (PBS) & Invitrogen (Auckland, New Zealand) \\
\hline Proliferation dye eFluor-670 & eBioscience (San Diego, CA, USA) \\
\hline Sodium azide powder & Sigma-Aldrich (St. Louis, MO, USA) \\
\hline Sodium chloride $(\mathrm{NaCl})$ & $\begin{array}{l}\text { BDH Laboratory Supplies } \text { (Poole, } \\
\text { England) }\end{array}$ \\
\hline Tris powder & Invitrogen (Auckland, New Zealand) \\
\hline Vancomycin hydrochloride hydrate & Sigma-Aldrich (St. Louis, MO, USA) \\
\hline
\end{tabular}




\subsubsection{Buffer and solution compositions}

\begin{tabular}{|c|c|}
\hline Antibiotic cocktail (Abx) & $\begin{array}{l}1 \mathrm{mg} / \mathrm{mL} \text { ampicillin } \\
1 \mathrm{mg} / \mathrm{mL} \text { gentamicin } \\
1 \mathrm{mg} / \mathrm{mL} \text { metronidazole } \\
1 \mathrm{mg} / \mathrm{mL} \text { neomycin } \\
0.5 \mathrm{mg} / \mathrm{mL} \text { vancomycin } \\
\text { sterile distilled } \mathrm{H}_{2} \mathrm{O}\end{array}$ \\
\hline Ammonium chloride Tris (ACT) buffer & $\begin{array}{l}0.144 \mathrm{M} \text { Ammonium chloride } \mathrm{pH} 7.4 \\
17 \mathrm{mM} \text { Tris } \mathrm{pH} 7.4\end{array}$ \\
\hline AutoMACS rinsing buffer & $\begin{array}{l}\text { 2mM EDTA } \\
\text { PBS }\end{array}$ \\
\hline AutoMACS running buffer & $\begin{array}{l}\text { 2mM EDTA } \\
1 \% \text { FCS } \\
\text { PBS }\end{array}$ \\
\hline Bicarbonate buffer, $\mathrm{pH} 9.6$ & $\begin{array}{l}0.1 \mathrm{M} \mathrm{Na}_{2} \mathrm{CO}_{3} \\
0.1 \mathrm{M} \mathrm{NaHCO} 3\end{array}$ \\
\hline Complete media (cIMDM) & $\begin{array}{l}\text { 1\% Penicillin-streptomycin } \\
55 \mu \mathrm{M} 2-\mathrm{ME} \\
5 \% \text { FCS } \\
\text { IMDM }\end{array}$ \\
\hline Digestion solution for gut samples & $\begin{array}{l}57.5 \mu \mathrm{g} / \mathrm{mL} \text { liberase } \mathrm{TL} \\
\text { IMDM }\end{array}$ \\
\hline Digestion solution for lymph node samples & $\begin{array}{l}100 \mu \mathrm{g} / \mathrm{mL} \text { liberase TL } \\
100 \mu \mathrm{g} / \mathrm{mL} \text { DNase I } \\
\text { IMDM }\end{array}$ \\
\hline Fluorescent activated cell sorting (FACS) buffer & $\begin{array}{l}\text { 10mM EDTA pH } 8.0 \\
0.01 \% \mathrm{NaN}_{3} \\
2 \% \mathrm{FCS} \\
\text { PBS }\end{array}$ \\
\hline Isolation buffer & $\begin{array}{l}0.1 \% \mathrm{w} / \mathrm{v} \text { BSA } \\
2 \mathrm{mM} \text { EDTA } \\
25 \mu \mathrm{g} / \mathrm{mL} \text { DNase I } \\
\text { PBS }\end{array}$ \\
\hline
\end{tabular}


Stripping buffer

2mM EDTA
$25 \mathrm{mM}$ Hepes buffer
$10 \%$ FCS
HBSS

\subsubsection{Antibodies}

Antibodies were purchased from BD Biosciences including BD Pharmingen ${ }^{\mathrm{TM}}$ and $\mathrm{BD}$ Horizon $^{\text {TM }}$ (San Diego, CA, USA), BioLegend (San Diego, CA, USA), eBioscience (San Diego, CA, USA), and R\&D Systems (Minneapolis, MN, USA) or purified and conjugated in-house.

\subsubsection{Flow cytometry}

All antibodies were titrated and the optimal dilution was determined. Before each use, antibodies were spun down to avoid adding antibody aggregates to samples. Antibody cocktails were made up in FACS buffer with appropriate dilutions and stored in the dark on ice until use.

\begin{tabular}{|c|c|c|c|}
\hline Antibody specificity & Conjugates & Clone & Supplier \\
\hline$\alpha 4 \beta 7$ (LPAM-1) & $\mathrm{PE}$ & DATK32 & BioLegend \\
\hline CCR7 & APC & $4 \mathrm{~B} 12$ & BioLegend \\
\hline CCR9 & FITC & 242503 & R\&D Systems \\
\hline CD4 & FITC & GK1.5 & BD Pharmingen \\
\hline $\mathrm{CD} 4$ & Pacific Blue & RM4-5 & BD Pharmingen \\
\hline CD11b & AF700, PerCРСy5.5 & M1/70 & BioLegend \\
\hline CD11c & PECy7 & HL3 & BD Pharmingen \\
\hline CD44 & APC-eFluor780 & IM7 & eBioscience \\
\hline CD45 & AmCyan & 2D1 & BD Biosciences \\
\hline CD45R (B220) & FITC & 6B2 & In-house \\
\hline CD45.1 & PECF594 & A20 & BD Horizon \\
\hline CD45.2 & APC, APC-eFluor780 & 104 & $\begin{array}{l}\text { Biolegend, } \\
\text { eBioscience }\end{array}$ \\
\hline CD64 & APC, PerCPCy5.5 & $\mathrm{X} 54-5 / 7.1$ & Biolegend \\
\hline
\end{tabular}




\begin{tabular}{|c|c|c|c|}
\hline CD69 & PerCРСy5.5 & $\mathrm{H} 1.2 \mathrm{~F} 3$ & Biolegend \\
\hline CD86 & FITC & GL-1 & BD Pharmingen \\
\hline CD103 & Biotin, PE & M290 & BD Pharmingen \\
\hline CXCR5 & Biotin & $2 G 8$ & BD Pharmingen \\
\hline Fc $\gamma$ RII/III (Fc Block) & - & $2.4 \mathrm{G} 2$ & In-house \\
\hline $\mathrm{F} 4 / 80$ & $\mathrm{PE}$ & BM-8 & eBioscience \\
\hline $\mathrm{IFN} \gamma$ & APC & XMG1.2 & BD Pharmingen \\
\hline IL-4 & PECy7 & 11B11 & Biolegend \\
\hline IL-17A & FITC & TC11-18H10.1 & Biolegend \\
\hline $\begin{array}{l}\text { Isotype } \operatorname{IgG1}\left(\mathrm{F}\left(\mathrm{ab}^{\prime}\right)_{2} \text { goat }\right. \\
\text { anti-human) }\end{array}$ & APC & - & $\begin{array}{l}\text { Jackson } \\
\text { ImmunoResearch } \\
\text { Laboratories (PA, } \\
\text { USA) }\end{array}$ \\
\hline Isotype $\operatorname{IgG} 2 \mathrm{a}$ & Biotin & R35-95 & BD Pharmingen \\
\hline Isotype IgG2b & APC & $27-35$ & BD Pharmingen \\
\hline MHCII & AF647, FITC & $3 \mathrm{JP}$ & In-house \\
\hline MHCII & APC-Cy7 & I-A/I-E & BioLegend \\
\hline OX40L & Biotin & RM134L & eBioscience \\
\hline Streptavidin & PE Texas-Red & Streptavidin & BD Pharmingen \\
\hline TIM-4 & $\mathrm{AF} 647, \mathrm{PE}$ & RMT4-54 & BioLegend \\
\hline$V \alpha 2$ & APC & B20.1 & eBioscience \\
\hline Vß5.1/5.2 & $\mathrm{PE}$ & MR9-4 & BD Pharmingen \\
\hline
\end{tabular}




\subsubsection{Viability dyes}

4,6-diamidino-2-phenylindole, dihydrochloride (DAPI) was purchased from Invitrogen (Auckland, New Zealand) and a working stock was prepared in FACS buffer at a concentration of $5 \mathrm{mg} / \mathrm{mL}$. DAPI was added to samples at a dilution of 1:25,000 just before analysis.

7-Amino-Actinomycin D (7-AAD) was purchased from BD Pharmingen (San Diego, CA, USA). A 1:80 dilution was added to samples just before analysis.

Live/dead fixable blue was purchased from BD Pharmingen (San Diego, CA, USA). Prior to use, dye was reconstituted in DMSO. A 1:200 dilution was added to samples. Staining was performed as per manufacturer's instructions.

Propidium Iodide (PI) was purchased from BD Pharmingen (San Diego, CA, USA). A 1:200 dilution was added to samples just before analysis.

\subsubsection{Mice}

\section{Maintenance and ethical approval}

Mice were bred and housed at the Malaghan Institute of Medical Research Biomedical Research Unit in Wellington, New Zealand. Sex matched mice of 6-8 weeks of age were used for all experiments. All protocols were approved by the Victoria University of Wellington Animal Ethics Committee. Protocol reference: 2012R4M

\section{Mouse strains}

C57BL/6J (C57) Breeding pairs were originally obtained from The Jackson Laboratory (Bar Harbour, ME, USA).

4C13R mice express the fluorescent reporters AmCyan and DS-Red for the cytokines IL-4 and IL-13 respectively. These mice were created using a bacterial artificial chromosome (BAC) clone spanning the il4 and il13 coding and regulatory regions. Reporter constructs 
were inserted into the appropriate regions of BACs by homologous recombination ${ }^{163}$. To check mice had inherited the reporter construct, all mice were tested by isolating DNA from tail samples and using PCR to target the DS-Red coding sequence. 4C13R mice were obtained from Dr. William E. Paul (National Institute of Allergic Diseases, National Institutes of Health, MD, USA).

B6-SLJ ptprc ${ }^{a}$ (CD45 congenic) mice express CD45.1 antigen on lymphocytes which can be differentiated from lymphocytes from a C57BL/6 (CD45.2) background. Breeding pairs were originally obtained from the Animal Resources Centre (Canning Vale, WA, Australia).

Toll-like receptor 4 knockout (TLR4-/-) mice are deficient for TLR4 and have been bred onto a C57 background ${ }^{164}$. Breeding pairs were obtained from the Research Institute of Microbial Diseases (Osaka University, Japan).

OTII mice express a transgenic CD4 $\mathrm{T}$ cell receptor specific for the MHCII restricted $\mathrm{OVA}_{323-339}$ peptide ${ }^{165}$. Mice were tail bled and tested for the transgenic receptor V $\alpha 2$ and V $\beta$ 5.1/5. by flow cytometry. OTII mice were obtained from Dr Sarah Hook (University of Otago, Dunedin, New Zealand).

OTII x B6-SJL ptprc $^{a}$ and OTII x 4C13R OT-II B6-SJL ptprca and OT-II 4C13R mice were obtained by crossing OTII mice with B6-SJL ptprca for one generation or with 4C13R mice for one generation at the Malaghan Institute of Medical Research BRU facility. OTII B6-SLJ ptprca mice were tested for inheritance of the transgenic receptor V $\alpha 2$ and V $\beta$ 5.1/5. by flow cytometry. In addition to being tested for inheritance of the transgenic $\mathrm{T}$ cell receptor, OT-II 4C13R were tested for inheritance of the reporter construct by PCR targeting the DS-Red coding sequence. 


\subsection{Methods}

\subsubsection{Mouse manipulations}

\subsubsection{Intragastric (i.g.) administration}

Solutions were administered to mice in a volume of $200-250 \mu \mathrm{L}$ via oral gavage using a $22 \mathrm{G}$ gavage feeding needle.

Intragastric administration of antigen

Mice were given $50 \mathrm{mg}$ OVA or BSA in a volume of $250 \mu \mathrm{L}$ in bicarbonate buffer via oral gavage.

To track OVA protein following i.g. administration, mice were given $250 \mu \mathrm{g}$ fluorescently labelled OVA (OVA-AF647) in a volume of $250 \mu \mathrm{L}$ in bicarbonate buffer via oral gavage .

\section{Intragastric administration of cholera toxin}

Mice were gavage fed $10 \mu \mathrm{g}$ of cholera toxin in $250 \mu \mathrm{L}$ bicarbonate buffer via oral gavage.

\section{Intragastric administration of antibiotics}

An antibiotic cocktail of $1 \mathrm{mg} / \mathrm{mL}$ ampicillin, gentamycin, metronidazole, neomycin and $0.5 \mathrm{mg} / \mathrm{mL}$ vancomycin was administered by oral gavage in $200 \mu \mathrm{L}$ sterile $\mathrm{H}_{2} \mathrm{O}$.

Intragastric administration of dextran sulfate sodium (DSS)

DSS was administered in the drinking water at $2.5 \% \mathrm{w} / \mathrm{v}$ for 3-5 days.

\subsubsection{Intravenous (i.v.) injection}

Injections were carried out with a $29 \mathrm{G}$ insulin needle into the lateral tail vein of restrained mice.

Injection of CD4+ OTII cells

To assess $\mathrm{T}$ cell responses following CT treatment, $5 \times 10^{6}$ splenic CD4+ OTII cells enriched by Dynabead selection (section 2.2.3.2) were injected in $200 \mu \mathrm{L}$ IMDM. 


\section{Injection of Brefeldin $A$}

In vivo cytokine production by $\mathrm{T}$ cells can be measured directly ex vivo using brefeldin $\mathrm{A}$, which prevents cytokine secretion and allows for the detection of cytokine stored inside the cell. Mice were injected with $250 \mu \mathrm{g}$ brefeldin A in PBS in $250 \mu \mathrm{L}$ and sacrificed 4 hours later.

\subsubsection{Euthanasia}

Mice were euthanised by $\mathrm{CO}_{2}$ asphyxiation or cervical dislocation.

\subsubsection{Tissue harvest and processing}

\subsubsection{Lymph nodes}

Inguinal, axillary, cervical and mesenteric (MLN) lymph nodes were removed from euthanised mice. Excess tissue was carefully removed and the lymph nodes were placed in IMDM.

\section{OTII cell isolation from lymph nodes}

Isolated lymph nodes from OTII x B6-SJ ptprca mice were flushed through a $70 \mu \mathrm{m}$ cell strainer using the rubber end of a syringe. The strainer was washed twice with $5 \mathrm{~mL}$ cIMDM. The samples were centrifuged for 10 minutes at $250 \mathrm{xg}$, supernatant discarded and the pellet disrupted by flicking. Cells were resuspended in 10mL cIMDM prior to CD4 Dynabead enrichment (section 2.2.3.2).

\section{Dendritic cell isolation from mesenteric lymph nodes}

Isolated MLN were broken up with a $25 \mathrm{G}$ needle in $1 \mathrm{~mL}$ of Liberase TL $(100 \mu \mathrm{g} / \mathrm{mL})$ and DNase $(100 \mu \mathrm{g} / \mathrm{mL})$ digest solution and incubated for 25 minutes at $37^{\circ} \mathrm{C}$. In the last 5 minutes of incubation EDTA was added at a final concentration of $10 \mathrm{mM}$. Cell were collected and filtered through a $70 \mu \mathrm{m}$ nylon cell strainer, centrifuged at $250 \mathrm{xg}$ and resuspended in $200 \mu \mathrm{L}$ IMDM. 


\subsubsection{Jejunum}

To isolate the jejunum, the small intestine was cut at $1.5 \mathrm{~cm}$ from the base of the stomach and the first $8 \mathrm{~cm}$ from the top of the section was collected for lamina propria isolation. The section was flushed with $10 \mathrm{~mL}$ of HBSS using an $18 \mathrm{G}$ needle and lightly pressed with forceps to remove intestinal waste, and excess mesenteric tissue and Peyer's patches were excised. The gut section was cut longitudinally and vigorously shaken in a 6 well plate with HBSS to remove mucus.

\section{Lymphocyte isolation from lamina propria}

To remove the epithelial cell layer, sections were placed in $5 \mathrm{~mL}$ pre-warmed stripping buffer (2.1.3) and vigorously vortexed before being incubated at $150 \mathrm{rpm}$ for 15 minutes at $37^{\circ} \mathrm{C}$ in a shaking incubator (Edison, NJ, USA). Following incubation, the samples were vortexed and transferred to a new tube with $5 \mathrm{~mL}$ pre-warmed stripping buffer and the process repeated. Samples were then rinsed in IMDM and transferred to a 6 well culture plate. Samples were finely chopped and $5 \mathrm{~mL}$ of pre warmed liberase TL digest solution was added to each sample at $57.5 \mu \mathrm{g} / \mathrm{mL}$. Tissue was disrupted using a $3 \mathrm{~mL}$ syringe and incubated at $37^{\circ} \mathrm{C}$ at $100 \mathrm{rpm}$ for 1 hour. To removed cell clumps, samples were filtered through a $40 \mu \mathrm{m}$ nylon filter prior to washing with IMDM. The cell pellet was resuspended in $200 \mu \mathrm{L}$ IMDM.

\subsubsection{Spleen}

Spleens were removed and kept on ice in IMDM until processing. Single cell suspensions were prepared by pressing spleens through a $70 \mu \mathrm{m}$ cell strainer with the end of a syringe and washed through with $20 \mathrm{~mL}$ IMDM. The cells were pelleted by centrifuging at $250 \mathrm{xg}$ for 10 minutes and the supernatant discarded. Cells were then incubated with $5 \mathrm{~mL}$ of ACT lysis buffer at $37^{\circ} \mathrm{C}$ to lyse red blood cells and washed with IMDM. The pellet was resuspended in $10 \mathrm{~mL}$ IMDM and an aliquot taken for counting.

\subsubsection{Thymus}

The thymus was removed from 4-5 week old mice and a single cell suspension was made by pressing the thymus through a $70 \mu \mathrm{M}$ cell strainer with the base of a syringe. Cells were washed through the cell strainer with $10 \mathrm{~mL}$ IMDM. 


\section{Labelling of thymocytes with eFluor670 dye}

Thymocytes were resuspended at $4 \times 10^{6}$ cells $/ \mathrm{mL}$ in PBS pre-warmed to $37^{\circ} \mathrm{C}$ and equal volume of $10 \mu \mathrm{M}$ eFluor670 in pre-warmed PBS was added to cell suspension while vortexing. Cells were incubated in the dark for 10 minutes at $37^{\circ} \mathrm{C}$ to incorporate the dye. The labelling reaction was stopped by adding 3x volume 100\% FCS and incubating on ice for 5 minutes. Cells were subsequently washed thrice with IMDM to remove any excess dye.

\section{Inducing apoptosis in thymocytes}

Thymocytes were incubated in $3 \mathrm{~mL} 10 \mu \mathrm{M}$ dexamethasone solution for 4 hours at room temperature. Apoptosis was confirmed by Annexin-V and PI staining.

\subsubsection{Counting of viable cells}

Cells were counted by trypan blue exclusion. Aliquots of single cell suspensions were diluted at 1:2 or 1:10 in Trypan Blue dye. $10 \mu \mathrm{L}$ of diluted cells were applied to a haemocytometer and the number of live cells was counted under a light microscope. Cells that excluded the dye were counted as viable.

\subsubsection{Cell purification}

\subsubsection{Magnetic cell separation (MACS)}

Cells were suspended at $2.5 \times 10^{8}$ cells $/ \mathrm{mL}$ in AutoMACS running buffer and filtered through $40 \mu \mathrm{m}$ cell strainer. MACS microbeads were added to the cell suspension at the appropriate dilution and incubated at $4^{\circ} \mathrm{C}$ for 15 minutes. Cells were washed with $2 \mathrm{~mL}$ AutoMACS running buffer and centrifuged at $250 \mathrm{xg}$ for 10 minutes. Cells were resuspended in $500 \mu \mathrm{L}$ AutoMACS running buffer and filtered through a $30 \mu \mathrm{M}$ MACS pre-separation filter before proceeding to magnetic separation on the AutoMACS machine (Miltenyi Biotec GmbH, Bergisch Gladbach, Germany).

\subsubsection{Dynabead purification}

Cells were resuspended at $1 \times 10^{8}$ cells $/ \mathrm{mL}$ in isolation buffer. $10 \mathrm{uL}$ of antibody per $1 \times 10^{7}$ cells was added and the sample and incubated for 10 minutes on ice. Cells were then washed 
and resuspended at $5 \times 10^{7}$ cells $/ \mathrm{mL}$ in isolation buffer. FlowComp ${ }^{\mathrm{TM}}$ dynabeads were added to the cell suspension, mixed well and incubated for 15 minutes at $4{ }^{\circ} \mathrm{C}$ with continuous agitation. Following incubation, samples were placed in an Invitrogen DynaMag ${ }^{\text {TM }}$ for 1 minute at room temperature and the supernatant was carefully removed and discarded. The cells and bound beads were resuspended in $1 \mathrm{~mL}$ isolation buffer and the process repeated. To remove the bound beads, cells were incubated with $1 \mathrm{~mL}$ FlowComp ${ }^{\mathrm{TM}}$ release buffer for 15 minutes at room temperature rolling and tilting. To remove the unbound beads, sample tubes were place in the DynaMag ${ }^{\text {TM }}$ and the supernatant was transferred to a new tube. Cells were spun down and resuspended in cIMDM.

\subsubsection{Cell staining}

\subsubsection{Cell surface staining}

$5 \times 10^{5}-5 \times 10^{6}$ cells/well were plated out onto a 96 well round bottom plate. Cells were resuspended in $100 \mu \mathrm{L}$ of Fc Block (2.4G2) and incubated for 10 minutes at $4^{\circ} \mathrm{C}$ and then centrifuged at $250 \mathrm{xg}$ for 2 minutes and supernatant discarded. Fluorecently labelled antibody cocktails were prepared using predetermined dilutions and $100 \mu \mathrm{L}$ of mixture was added to cells followed by incubation at $4^{\circ} \mathrm{C}$ for 20 minutes before being washed twice with $100 \mu \mathrm{L}$ FACS buffer. For secondary antibody staining, cells were incubated at $4^{\circ} \mathrm{C}$ for a further 20 minutes with the diluted antibody and washed twice with FACS buffer. After the final wash, samples were resuspended in $200 \mu \mathrm{L}$ FACS buffer containing viability dye (section 2.2.4.3.) and transferred to microtubes for immediate flow cytometric analysis.

\section{Cell surface staining of $C X_{3} C R 1$}

To stain cell surface $\mathrm{CX}_{3} \mathrm{CR} 1$, cells were plated out into 96 well plates and blocked with $100 \mu \mathrm{L} \mathrm{10 \%} \mathrm{goat} \mathrm{serum} \mathrm{for} 30$ minutes at $4{ }^{\circ} \mathrm{C}$. Cells were spun down and resuspended in $50 \mu \mathrm{L} \mathrm{CX} \mathrm{CX}_{3} \mathrm{CR} 1-\mathrm{Fc}$ fusion protein NTN and incubated for 30 minutes at $4^{\circ} \mathrm{C}$. Cells were washed twice with cold FACS buffer and resuspended in $50 \mu \mathrm{L}$ of the secondary antibody, goat anti-human IgG1-APC. and incubated for a further 30 minutes at $4{ }^{\circ} \mathrm{C}$, cells were washed twice with cold FACS buffer and stained for other cell surface antigens. 


\subsubsection{Intracellular cytokine staining}

Cells isolated from brefeldin A-treated mice were stained with live/dead fixable blue (section 2.2.4.3.) and cell surface markers (section 2.2.4.1.). Cells were then assessed for intracellular cytokines with a BD Cytofix/Cytoperm ${ }^{\mathrm{TM}}$ kit following the manufacturer's protocol. Briefly, cells were fixed in cytofix/cytoperm solution for 20 minutes at $4^{\circ} \mathrm{C}$, and then washed twice with Perm/Wash ${ }^{\text {TM }}$ buffer. Antibodies targeting cytokines were diluted in Perm/Wash ${ }^{\text {TM }}$ buffer and added to cells for 30 minutes at $4^{\circ} \mathrm{C}$. Cells were washed thrice and resuspended in FACS buffer prior to flow cytometric analysis.

\subsubsection{Detection of retinal dehygrogenase with ALDEFLUOR kit}

To assess retinal dehydrogenase (RALDH) activity, cells were stained for ALDEFLUOR, a lipid soluble substrate the fluroesces upon interaction with aldehyde dehydrogenases. $1 \mu \mathrm{L}$ activated ALDEFLUOR was added to the cell suspension and mixed well. To prepare a negative staining control an aliqout of sample was removed and $5 \mu \mathrm{L}$ of the specific aldehyde dehydrogenase inhibitor diethylaminobenzaldehyde (DEAB) was immediately added to control samples. Cells were incubated for 20 minutes at $37^{\circ} \mathrm{C}$ to allow for the reaction to take place. Following incubation, cells were pelleted at 250xg for 5 minutes. Cell were then stained with antibodies against cell surface antigens (section 2.2.4.1.) which was carried out in ALDEFLUOR assay buffer to prevent ALDEFLUOR efflux.

\subsubsection{Viability dye staining}

Just prior to flow cytometic analysis DAPI or 7-AAD or PI was added to stained samples in FACS buffer at the appropriate dilution (section 2.1.5.)

\section{Fixable viability dye staining}

Fixable viability dyes were added to cell suspension at the appropriate dilution in FACS buffer. Cells were incubated at $4^{\circ} \mathrm{C}$ for 30 minutes and washed prior to cell surface staining. 


\subsubsection{Flow cytometry}

\subsubsection{Controls}

Unstained cells, single stained compensation beads and fluorescence minus one (FMO) stained cells were used as controls for all experiments. Isotype antibody staining controls were used when necessary to control for background fluorescence.

\subsubsection{Acquisition and analysis of data}

Samples were filtered through a $40 \mu \mathrm{m}$ cell strainer and run on a LSRFortessa ${ }^{\mathrm{TM}}$ or a LSR II flow cytometer (BD Biosciences, San Jose, CA, USA) using FACS Diva software. A minimum of $1 \times 10^{6}$ events were collected for jejunal samples and a minimum of $2.5 \times 10^{6}$ events were collected for MLN samples. Flow rate was maintained below 5000 events per second.

Following acquisition, all data was analysed using Flowjo software (version 9.7). Doublets gated out using forward and side scatter parameters and dead cells excluded as indicated by viability dye staining prior to any additional analysis. Full gating strategies can be found in Appendix I and II.

\subsubsection{Assays of cell function}

\subsubsection{In vitro phagocytosis assay}

Purified CD11c+ cells from the LP or MLN were incubated for 1 hour at $37^{\circ} \mathrm{C}$ with e670 labelled or unlabelled apoptotic thymocytes at a ratio of 1:10. Following incubation, cells were harvested and stained for analysis by flow cytometry. Phagocytic ability was determined by e670 incorporation by CD11c+ cell subsets.

\subsubsection{Statistical analysis}

Data was analysed using Prism software and means \pm standard error of the mean are displayed on bar graphs. P-values of $<0.05$ from a Student's unpaired t-test, One-Way ANOVA test or Two-Way ANOVA test were classified as being statistically significant. 


\section{Chapter 3: Phenotypic characterisation of intestinal APC subsets at steady-state}




\subsection{Introduction}

APC in the intestine are exposed to vast amounts of foreign antigen and play an important role in the prevention of inappropriate immune responses to food proteins and commensal bacteria ${ }^{42,55,111}$. For this reason, intestinal APC differ from those found in other tissues and common markers used to define APC populations may not be applicable in the intestine. Interestingly, despite the importance of intestinal APC in maintaining immune homeostasis, there are still many unanswered questions about the composition and functions of the highly heterogeneous intestinal APC population at steady-state.

Intestinal APC, identified by co-expression of CD11c and MHCII, are commonly classified into two broad populations based on their expression of the non-overlapping markers CD103 and $\mathrm{CX}_{3} \mathrm{CR} 1$ (see Section 1.3) ${ }^{42,101}$. However, there is increasing evidence that this classification fails to highlight the highly heterogeneous nature of intestinal APC populations. While co-expression of CD11c and MHCII is useful in identifying DC populations in other tissues ${ }^{37}, \mathrm{CD} 11 \mathrm{c}+\mathrm{MHCII}+$ cells from the intestine consist of a mixture of DC and $\mathrm{M} \varphi{ }^{101,105}$. CD103+ cells are phenotypically similar to DC ${ }^{42}$, whereas, $\mathrm{CX}_{3} \mathrm{CR} 1+$ cells are usually considered to be closely related to $\mathrm{M} \varphi{ }^{101,105}$ due to their expression of the $\mathrm{M} \varphi$ markers F4/80 ${ }^{114}$ and CD $64{ }^{113}$, lack of expression of the DC specific protein zBTB46 ${ }^{115}$ and the presence of vacuoles in their cytoplasm ${ }^{101}$. However, recent studies using GFP-reporter mice have shown a proportion of $\mathrm{CX}_{3} \mathrm{CR} 1+$ cells express $\mathrm{DC}$ specific protein zBTB46, suggesting that the classification of all of these cells as M $\varphi$ may not be appropriate ${ }^{103}$. Furthermore, there are subsets of CD11b+ and CD11b- cells within the CD103 and $\mathrm{CX}_{3} \mathrm{CR} 1$ populations ${ }^{101}$ (see Section 1.3.) and there is a population of CD11c+ MHCII+ cells that lack expression of both CD103 and $\mathrm{CX}_{3} \mathrm{CR} 1$. Understanding composition of intestinal APC subsets is further complicated by the fact that different labs use different mouse strains ${ }^{75,166}$ and use various intestinal processing protocols, which may or may not exclude Peyer's patches ${ }^{101}$, for their studies. In addition, sections of the intestine have very distinct APC compositions, therefore, study of different intestinal sections may yield very different results ${ }^{80}$. Therefore, due to the discordance among published studies in relation to the composition of intestinal APC and the underappreciated heterogeneity of 
APC populations, I sought to develop an improved strategy of characterising jejunal APC to gain a clear understanding of the subsets present at steady-state.

In addition to APC phenotype and subset composition, I was interested in assessing some functional aspects of intestinal APC populations at steady-state. In order to initiate T cell responses, APC are required to take up antigen in the tissue and migrate to the draining lymph node where they can present antigen to $\mathrm{T}$ cells ${ }^{9}$. While these processes are well understood in other tissues, they remain an enigma in the intestine. This is because $\mathrm{CX}_{3} \mathrm{CR} 1+$ cells localise to the epithelial barrier ${ }^{38,42,80}$ where they are thought to be responsible for the uptake of luminal antigen through transepithelial dendrites ${ }^{71,75,76}$. However, $\mathrm{CX}_{3} \mathrm{CR} 1+$ cells are non-migratory and poor at inducing $\mathrm{T}$ cell proliferation ${ }^{42,101}$. On the other hand, CD103+ cells preferentially localise to the centre of villi and experiments have failed to demonstrate that $\mathrm{CD} 103+\mathrm{DC}$ have the capacity to take up antigen in vivo. Nevertheless, CD103+ DC migrate to the MLN in a CCR7 dependent manner ${ }^{111}$ and are potent drivers of $\mathrm{T}$ cell proliferation to orally delivered antigen ${ }^{101,108}$. Thus, a unique situation appears to exist in the intestine where antigen transfer may be required to take place between the $\mathrm{CX}_{3} \mathrm{CR} 1$ and $\mathrm{CD} 103$ populations to allow for antigen presentation. A recent study suggests that antigen transfer may occur between these populations via gap junctions ${ }^{56}$. However, despite these recent findings, it remains unclear whether distinct subsets within the $\mathrm{CX}_{3} \mathrm{CR} 1+$ population are responsible for antigen uptake or whether antigen uptake is a function of all $\mathrm{CX}_{3} \mathrm{CR} 1+$ cells. Therefore, I wanted to assess antigen uptake among intestinal APC subsets as defined using the method described in this Chapter.

Intestinal DC drain to the MLN where they can interact with $\mathrm{T}$ cells and influence immune responses. Therefore, the MLN consists of migratory DC populations originating from the LP and lymph node resident DC. Under homeostatic conditions, DC from the MLN can be categorised into four populations based on cell surface expression of CD103 and CD11b. Of these subsets, CD103+ CD11b+ cells are unique to the MLN and have a similar phenotype to DC reported in the LP. Therefore, it is likely these DC originated in the LP and migrated to the MLN ${ }^{109}$. In addition, CD103+ CD11b- cells are thought to originate in the Peyer's 
patches, isolated lymphoid follicles and possibly the LP and are therefore likely to be contributing to the CD103+ CD11b- subset in the MLN ${ }^{101}$. Both CD103+ CD11b+ and CD103+ CD11b- populations have the capacity to present orally delivered antigen ${ }^{109}$ and are required to drive Treg development through production of chemical mediators such as retinoic acid (RA) and TGF- $\beta^{57,58}$. CD103+ DC populations are specifically equipped to express the enzyme retinal dehydrogenase (RALDH) which allows for the conversion of dietary vitamin A derivatives to RA. In this context, RA is not only important for driving the development of Tregs in the MLN but also for the upregulation of gut homing receptors such as CCR9 and $\alpha 4 \beta 7$ that facilitate the entry of T cells into the LP ${ }^{57,167,168}$. In contrast, CD103- CD11b+ and CD103- CD11b- lymph node resident populations lack the ability to present oral antigen and promote upregulation of CCR9 ${ }^{109}$. These observations would suggest that only DC populations that have originated in the LP have the capacity to regulate intestinal homeostasis. However, it is unclear whether distinct subsets within the CD103+ population migrate at steady-state and are responsible for Treg induction or whether this is a characteristic of all CD103+ DC. Furthermore, it is not clear whether other LP resident DC have the capacity to migrate to the MLN. Therefore, I sought to assess the composition of DC subsets present in the MLN at steady-state using the same strategy used to assess LP APC.

In order to gain a better understanding of the phenotype and function of intestinal APC I analysed APC from the LP and MLN of naïve mice for expression of a range of DC and M $\varphi$ markers. In addition to the classical markers for identifying DC, I chose to assess markers associated with $\mathrm{M} \varphi$ such as F4/80 and TIM-4 and other non-conventional DC populations such as CD64. My results showed that LP CD11b+ APC could be divided into three distinct subsets, including CD103+ TIM-4- DC, CD103- TIM-4+ $\mathrm{M} \varphi$ and a heterogeneous population of CD103- TIM-4- cells. In addition, I found LP M $\varphi$ to express $\mathrm{CX}_{3} \mathrm{CR} 1, \mathrm{~F} 4 / 80$ and CD64 and are highly efficient at antigen uptake in vivo whereas LP DC showed high activity of RALDH and appear to constitute a migratory $\mathrm{MHCII}^{\mathrm{hi}} \mathrm{DC}$ population in the MLN. Therefore, assessing intestinal APC for expression of CD103 and TIM-4 may be a useful method to assist in the identification of distinct APC populations in the intestine. 


\subsection{Aims}

Since the establishment of two broad CD11c+ MHCII+ subsets in the intestinal LP, further study of these subsets has been limited. Study of splenic, skin and lung resident DC has shown that there are many subsets of DC, each with distinct functions ${ }^{169,170,171,172}$. This chapter summarises my characterisation of intestinal DC and $\mathrm{M} \varphi$ at steady-state.

My specific aims were:

1. To assess LP and MLN APC for expression of a range of cell surface molecules to determine if established populations could be further divided into distinct subsets.

2. To investigate functional characteristics of APC subsets in terms of capacity to metabolise retinoic acid as determined by activity of RALDH and capacity for antigen uptake in vivo. 


\subsection{Results}

\subsubsection{LP APC phenotype and subset compositions at steady-state}

Previous studies have found the composition of LP APC subsets to differ significantly from other tissues. Therefore, I was interested to determine the phenotype of small intestinal LP resident APC subsets at steady-state. The small intestine is divided into three histologically distinct sections; the duodenum, the jejunum and the ileum that can vary in length between mice ${ }^{173}$. The jejunum and proximal ileum are the most commonly studied sections from the small intestine in mice ${ }^{42,101,102}$ and share similar APC compositions ${ }^{102}$. For consistency I focused solely on APC isolated from a section of the jejunal LP in this study. Since I was unable to analyse histological sections for each specimen studied, our lab adopted a strategy that defines small intestinal sections by measurement. The duodenum is defined as the first $1.5 \mathrm{~cm}$ of intestine beginning at the base of the stomach. The jejunum begins at the end of the duodenum and included the next $8 \mathrm{~cm}$ of intestine. Lastly, the ileum is taken proximal to the cecum and includes the next $8 \mathrm{~cm}$ of intestine above. To prevent lymphoid cell contamination, Peyer's patches were excised from each section prior to analysis.

APC isolated from the jejunal sections of naïve mice were identified by co-expression of CD11c and MHCII and found to account for approximately $20 \%$ of the total cellularity in the LP (Fig. 3.1, A). I found CD11c+ MHCII+ cells could be divided into four subsets based on cell surface expression of CD103 and CD11b. Approximately 90\% of CD11c+ MHCII+ cells were CD11b+ among which 25\% were CD103+. The full gating strategy I used to identify intestinal APC can been found in Appendix I. Interestingly, I found expression of the activation markers MHCII and CD86 to be significantly higher on CD103CD11b+ APC compared to other APC subsets at steady-state (Fig. 3.2). This data shows LP resident APC are predominantly made up of $\mathrm{CD} 11 \mathrm{~b}+$ cells at steady-state which can be further divided into CD103+ and CD103- subsets. 
A
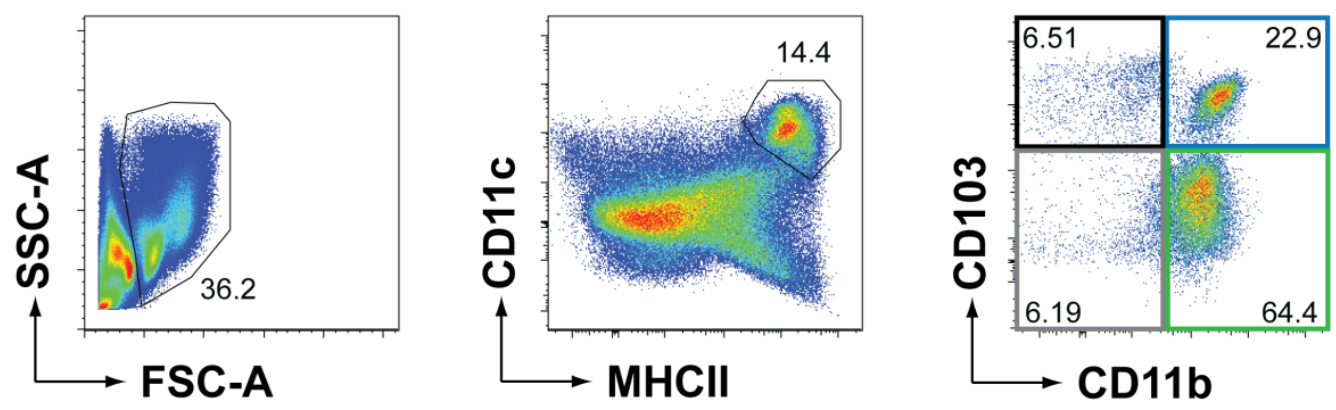

B

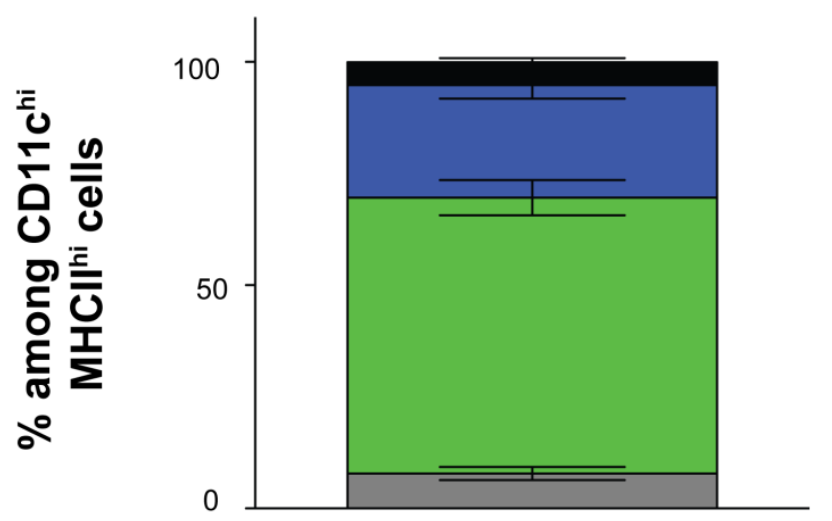

- CD103+ CD11b-

CD103+ CD11b+

CD103- CD11b+

CD103- CD11b-

Figure 3.1: APC subsets from the jejunal LP

Cells isolated from the jejunal LP of naïve mice were stained and analysed by flow cytometry. DC were identified by CD11c and MHCII staining. DC were divided into subsets based on cell surface expression of CD103 and CD11b. Frequency of DC subsets are shown on FACS dot plots (A) and bar graph (B). Graph shows mean \pm SEM of four independent experiments each including 3 mice per group. 


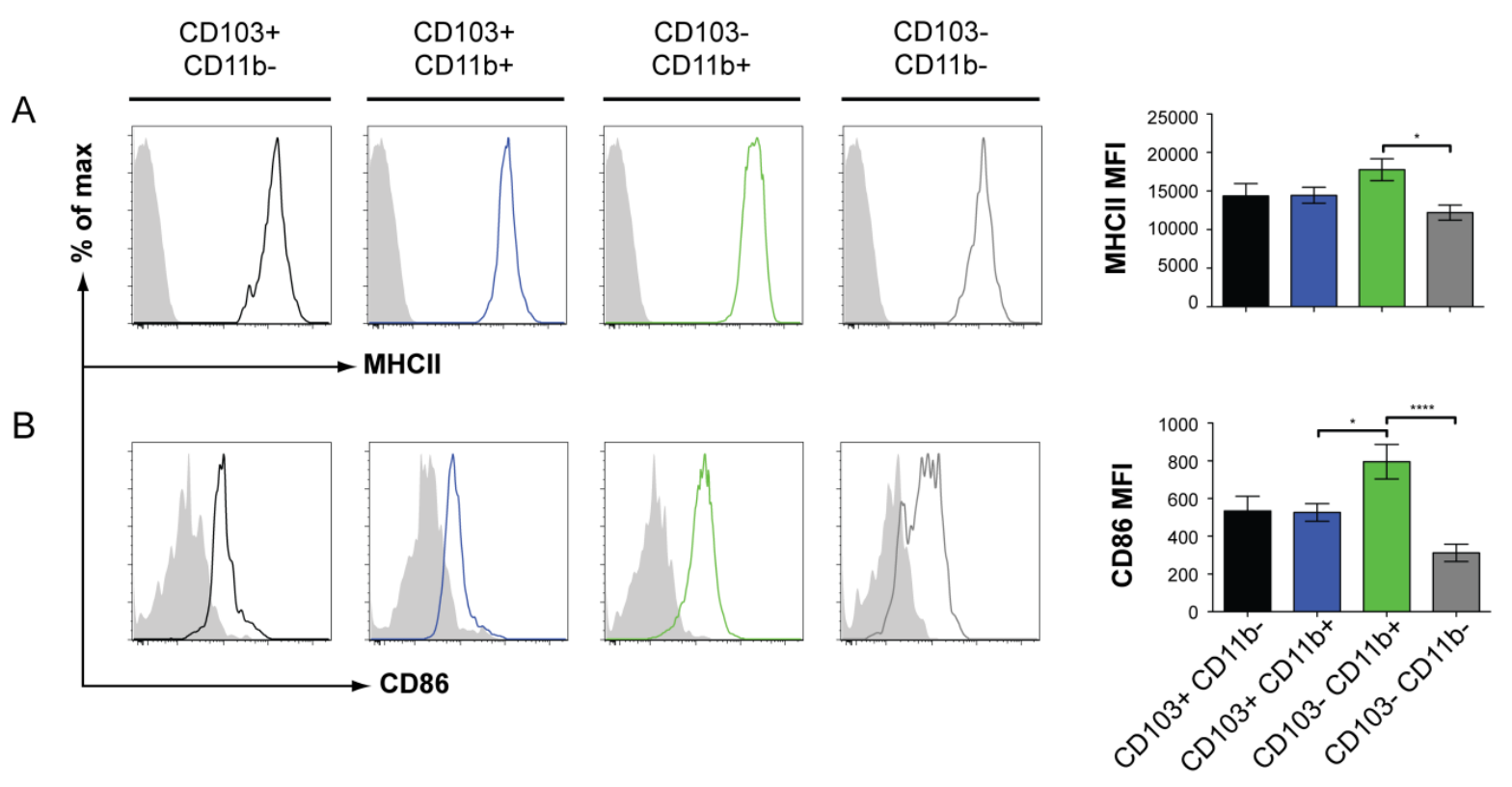

Figure 3.2: Activation status of LP APC subsets

APC isolated from the jejunal LP of naïve mice were examined for activation marker expression by flow cytometry. MHCII (A) and CD86 (B) staining is shown on histograms for individual DC subsets (coloured line) as compared to FMO controls (shaded). Quantification was determined by MFI as shown on bar graphs. Bar graphs show combined mean \pm SEM of four independent experiments each with 3 mice per experimental group. Statistical significance was determined by One-Way ANOVA with Bonferroni post-test. $* \mathrm{p}<0.05,{ }^{* * * *} \mathrm{p}<0.0001$. 


\subsubsection{CD103 and TIM-4 expression clearly defines three populations of CD11b+ APC in the LP at steady-state}

Next I was interested to further characterise CD11b+ CD103+/- populations from the LP. To do this, I isolated APC from the jejunal LP of naïve mice and analysed them for the expression of a range of cell surface markers including CD11c, $\mathrm{CX}_{3} \mathrm{CR} 1, \mathrm{~F} 4 / 80, \mathrm{CD} 64$ and TIM-4. From these studies, I found CD103- APC populations to have lower expression of DC marker CD11c compared to CD103+ APC populations (Fig. 3.3, A). Furthermore, CD103- CD11b+ cells, but not CD103+ cells expressed the $\mathrm{M} \varphi$ markers $\mathrm{CX}_{3} \mathrm{CR} 1, \mathrm{~F} 4 / 80$ and CD64 (Fig. 3.3., B-D). The CD103- CD11b- population showed intermediate expression of F4/80 and had bimodal expression of CD64. Interesting, I also found that CD103CD11b- cells had bimodal expression of TIM-4. Therefore, I analysed CD103- CD11bCD64+ and CD103- CD11b- CD64- populations separately for expression of TIM-4. I found approximately 75\% of CD103- CD11b- CD64+ cells expressed TIM-4 compared to 35\% of CD103- CD11b- CD64- cells. Furthermore, I found the CD103- CD11b+ APC population contained TIM-4+ and TIM-4- cells whereas CD103+ APC showed little to no expression of TIM-4 (Fig. 3.3, E).

Expression of TIM-4 appeared to highlight two distinct populations within the CD103populations. As CD103- CD11b+ cells constituted a significant population of LP resident APC at steady-state, I was interested to further characterise TIM-4+ and TIM-4- cells within this population to determine if there were additional phenotypic differences. TIM-4 was not found to be expressed on CD103+ populations at steady-state (Fig. 3.4, A-B), therefore, I hypothesised that TIM-4 expression may be associated with intestinal M $\varphi$. Analysis by flow cytometry revealed CD103- CD11b+ TIM-4+ (CD103- TIM-4+) cells to have significantly higher expression of $\mathrm{M} \varphi$ marker $\mathrm{CX}_{3} \mathrm{CR} 1$ compared to CD103- CD11b+ TIM-4- (CD103TIM-4-) cells (Fig. 3.4, C). Furthermore, approximately $80 \%$ and $100 \%$ of CD103- TIM-4+ cells express F4/80 and CD64 respectively. In comparison only 45\% F4/80+ and 60\% CD64+ cells were detected among CD103- TIM-4- cells (Fig. 3.4, D-E). In summary, this data shows cell surface expression of CD103 and TIM-4 can be used to clearly identify three 
phenotypically distinct populations among CD11b+ LP resident APC (CD103+ TIM-4-, CD103- TIM-4+ and CD103- TIM-4-). 


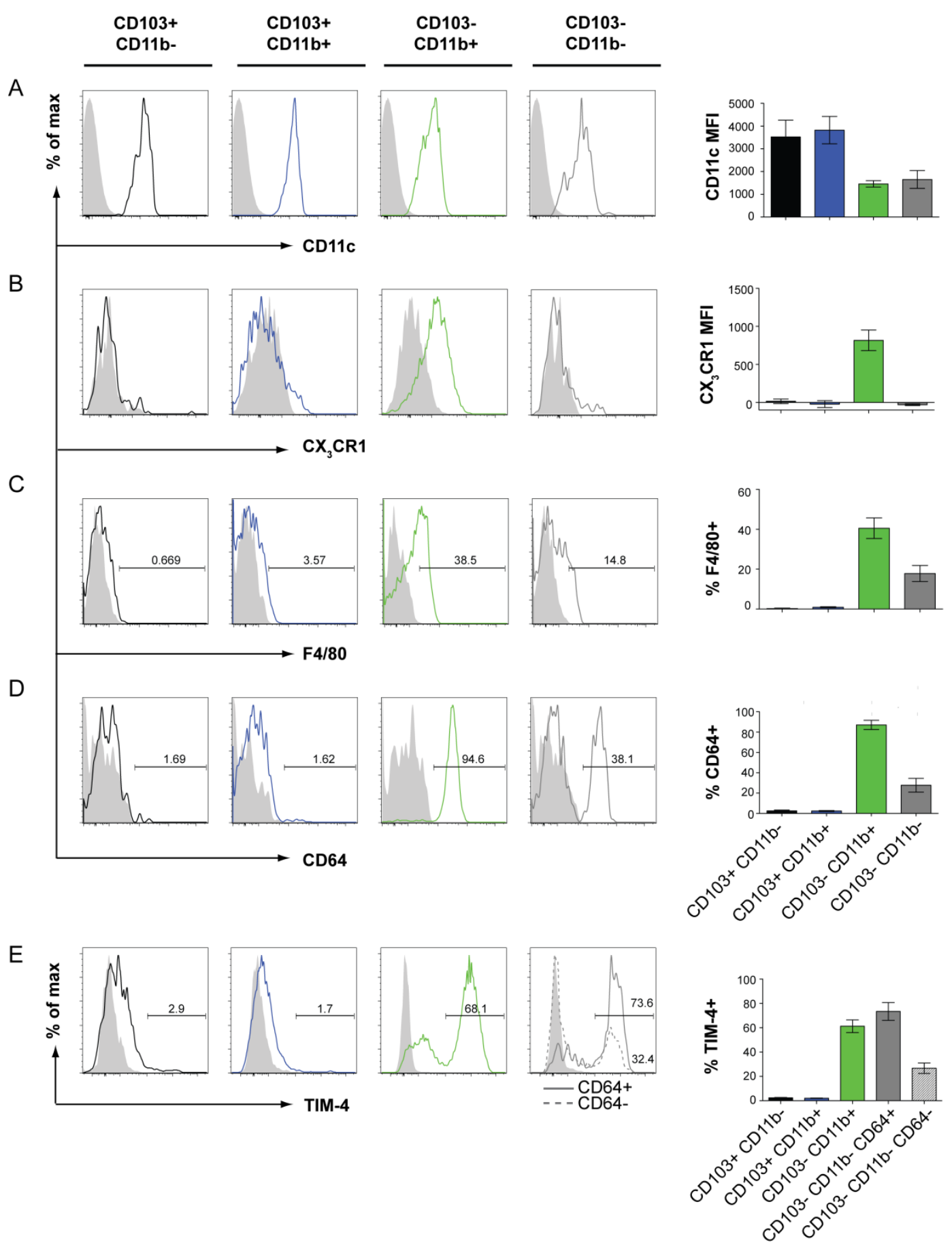

Figure 3.3: Cell surface marker expression on LP APC

DC isolated from the jejunal LP of naïve mice were assessed for cell surface marker expression by flow cytometry. CD11c (A), CX 3 CR1 (B), F4/80 (C), CD64 (D) and TIM-4 (E) staining is shown on histograms for individual DC subsets (coloured line) compared to FMO or isotype controls (shaded). Bar graphs show MFI or the frequency of positive cells within each DC subset. Graphs show mean \pm SEM of two to four independent experiments, each with 3 mice per experimental group. 
A
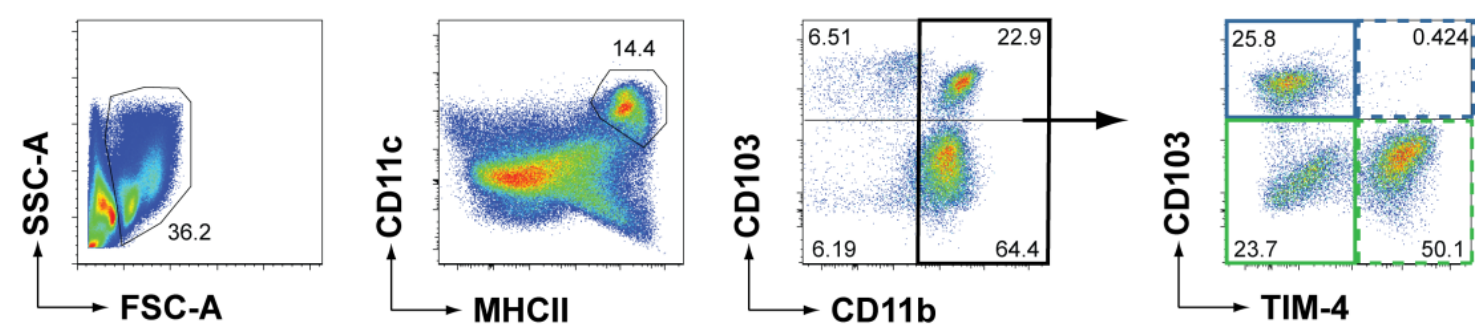

B

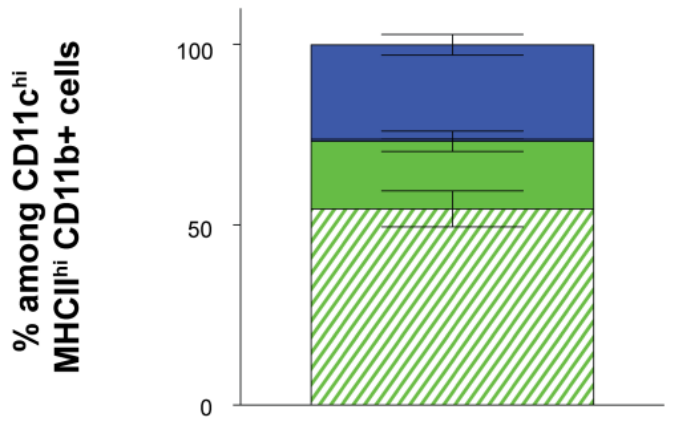

CD103+ TIM-4-

!: CD103+ TIM-4+

CD103- TIM-4-

I-- CD103- TIM-4+
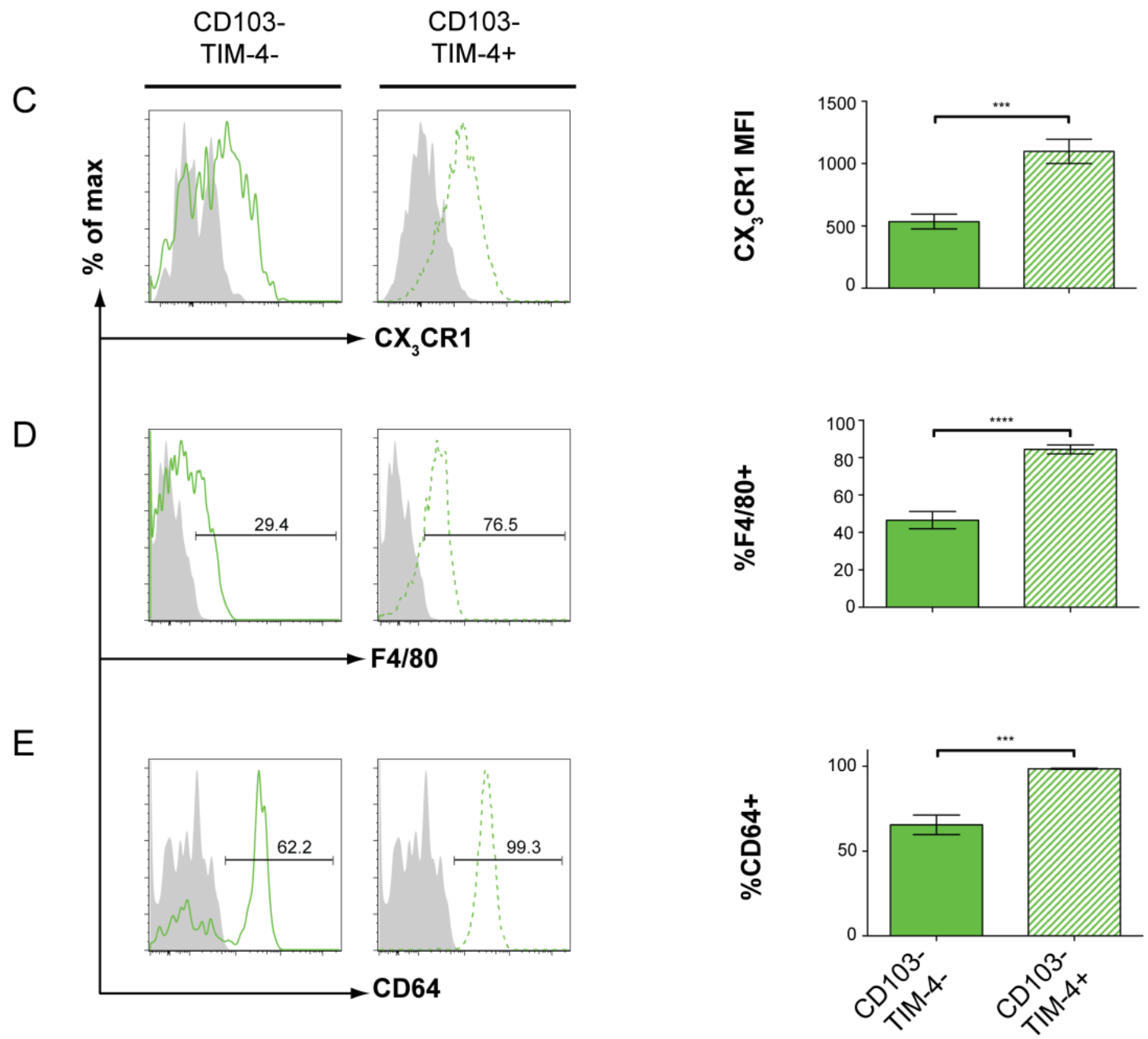
Figure 3.4: CD103- CD11b+ APC can be divided into two distinct populations

DC were isolated from the jejunal LP of naïve mice and CD11b+ DC were assessed for cell surface expression of CD103 and TIM-4. Frequency of DC subsets among total CD11b+ CD11c+ MHCII+ cells are shown on pseudo colour plots (A) and stacked bar graph (B). Histograms are gated on CD11b+ CD103- TIM-4- (green line, left column) and CD11b+ CD103- TIM-4+ (green dashed line, right column) and show expression of CXCR1 (C), F4/80 (D) and CD64 (E). All surface marker expression is compared to FMO or isotype controls (shaded areas). Bar graphs display MFI or frequency of positive cells within each subset. Graphs show mean \pm SEM of two to four independent experiments, each with 3 mice per experimental group Statistical significance was determined by a Student's unpaired t-test. ${ }^{* * *} 0.0001<\mathrm{p}<0.001, * * * * \mathrm{p}<0.0001$ 


\subsubsection{CD103- TIM-4+ APC take up antigen at steady-state}

$\mathrm{CX}_{3} \mathrm{CR} 1+\mathrm{M} \varphi$ have been shown to sample antigen directly from the intestinal lumen ${ }^{75}$. I found CD103- TIM-4+ cells to be phenotypically similar to reported $\mathrm{CX}_{3} \mathrm{CR} 1+\mathrm{M} \varphi$. Therefore, I was interested to determine whether CD103- TIM-4+ cells shared functional characteristics of $\mathrm{CX}_{3} \mathrm{CR} 1+\mathrm{M} \varphi$. To address this, mice were gavage fed OVA labelled with AF647 in combination with cholera toxin (CT). APC were isolated from the LP one hour later and assessed for AF647 fluorescence. I found that antigen was predominantly taken up by CD103- APC (Fig. 3.5, A). Closer examination revealed approximately 8\% of CD103TIM-4+ cells were positive for OVA-AF647 antigen compared to 1.5\% of CD103- TIM-4cells. Furthermore, minimal AF647+ cells were found among other LP resident APC subsets (Fig 3.5, B-C). This data suggests the CD103- TIM-4+ subset is the most efficient of the LP resident APC subsets in taking up luminal antigen at steady-state. 
A

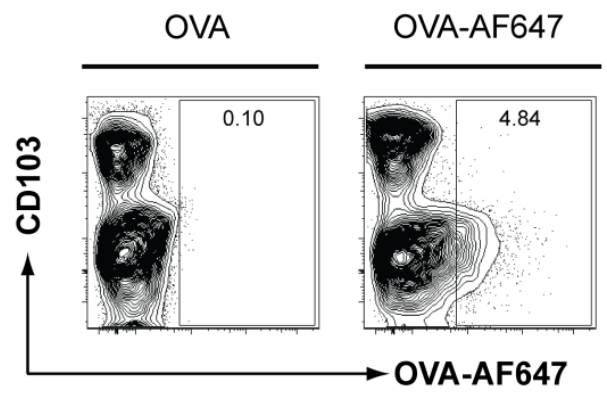

B

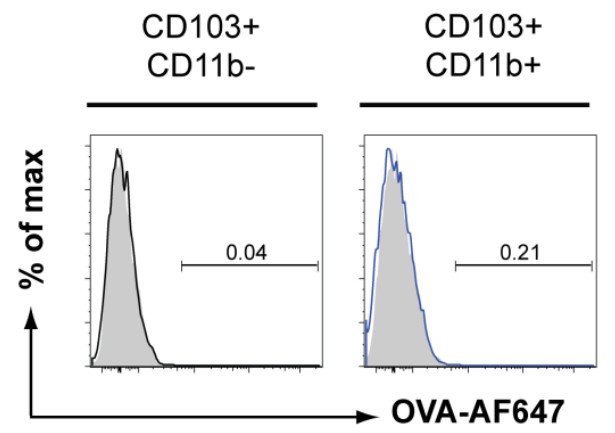

C

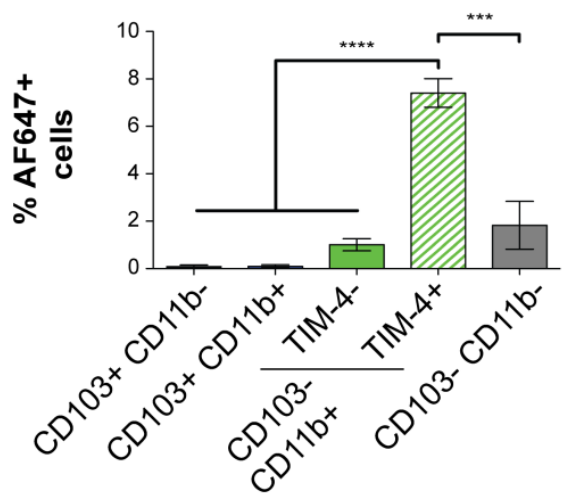

CD103CD11b+ TIM-4- CD11b+ TIM-4+ CD11b-
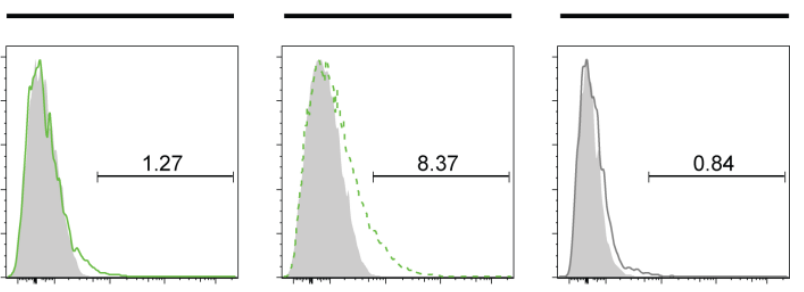

Figure 3.5: CD11b+ TIM-4+ APC take up antigen at steady-state

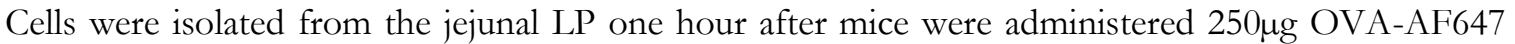
with $10 \mu \mathrm{g}$ cholera toxin (CT) by oral gavage. Uptake of OVA-AF647 by LPDC populations was analysed by flow cytometry. A) Contour flow plots show OVA-AF647 staining among CD11b+ CD11c+ MHCII+ cells. B) Histograms show expression of OVA -AF647 by DC subsets. (C) Bar graphs show frequency of AF647+ among individual DC subsets. Graph shows mean \pm SEM. $n=3$. Statistical significance was determined by One-Way ANOVA with Bonferroni post-test. *** $0.0001<\mathrm{p}<0.001,{ }^{* * * *} \mathrm{p}<0.0001$. Data is representative of three independent experiments. 


\subsubsection{CD103+ CD11b + LP APC have high activity of RALDH at steady-state}

RALDH dependent RA metabolism by CD103+ DC has been reported to be important for

driving differentiation of Tregs at steady-state ${ }^{57,58}$. I used an ALDEFLUOR ${ }^{\mathrm{TM}}$ assay kit to assess the activity of RALDH among LP resident APC subsets. The ALDEFLUOR ${ }^{\text {TM }}$ substrate fluoresces upon interaction with the active RALDH enzyme, allowing for quantitative analysis of enzyme activity by flow cytometry. The RALDH inhibitor DEAB is added to control samples to determine background fluorescence. I found that CD103+ cells show the highest RALDH activity (Fig. 3.6, A). Among the CD103+ compartment, CD103 + CD11b + DC had significantly higher frequency of cells expressing active RALDH compared to CD103+ CD11b- DC (Fig. 3.6, B-C). Interestingly, among CD103- CD11b+ cells I found TIM-4+ cells to show no RALDH activity whereas the TIM-4- cells showed modest RALDH activity, with up to $30 \%$ of cells expressing active RALDH. These results show that CD103+ CD11b+ DC have significantly higher frequencies of cells expressing RALDH, however, other LP resident APC populations also have some RALDH activity at steady-state. 
A

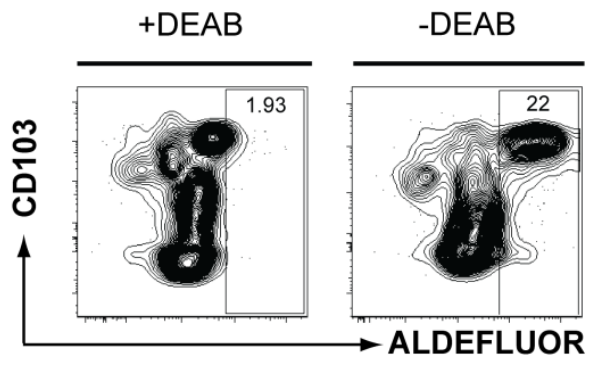

$\mathrm{B}$

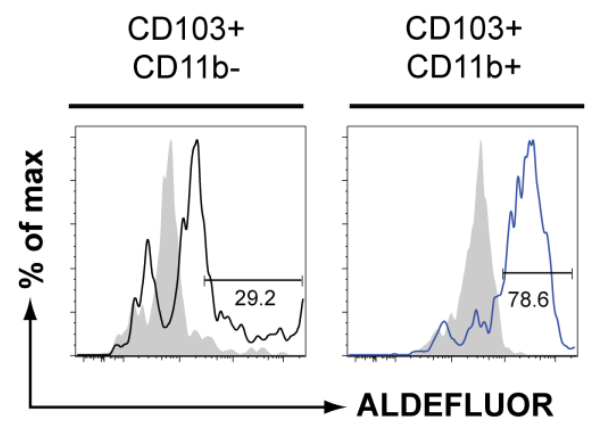

C

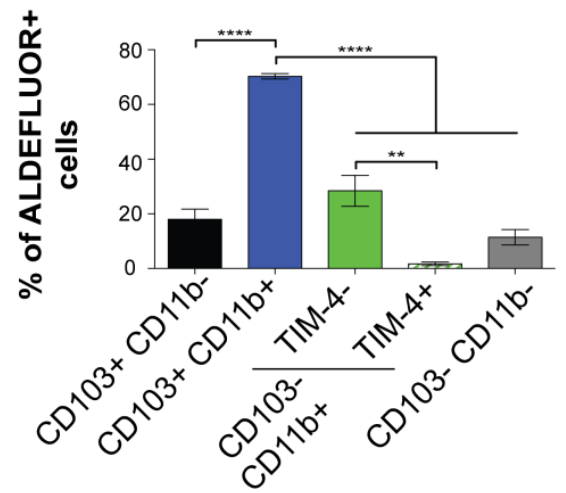

CD103CD11b+ TIM-4-
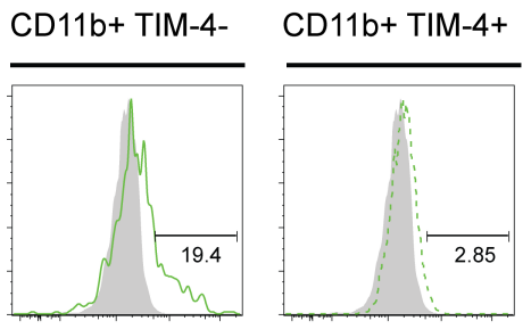

CD103CD11b-

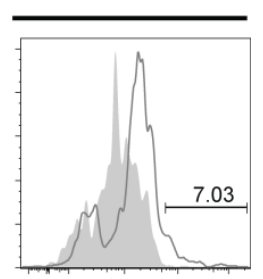

\section{Figure 3.6: CD103+ LPDC have active RALDH at steady-state}

Cells isolated from the jejunal LP of naïve mice were assessed for RALDH expression using an ALDEFLUOR staining kit. (A) Contour flow plots show ALDEFLUOR staining among CD11b+ $\mathrm{CD} 11 \mathrm{c}+\mathrm{MHCII}+$ cells compared to cells treated with RALDH inhibitor diethylaminobenzaldehyde (DEAB). B) Histograms show ALDEFLUOR staining for individual DC subsets compared to DEAB treated control cells. C) Bar graph shows frequency of ALDEFLUOR+ cells within each DC subset. Graph shows mean \pm SEM. $n=3$. Statistical significance was determined by One-Way ANOVA with Bonferroni post-test. ${ }^{* *} 0.001<\mathrm{p}<0.05$, ${ }^{* * *} 0.0001<\mathrm{p}<0.001,{ }^{* * * *} \mathrm{p}<0.0001$. Data is representative of four independent experiments. 


\subsubsection{MLN DC phenotype and subset compositions at steady-state}

LP APC acquire antigen and migrate to the MLN where they present antigen to T cells and initiate appropriate immune responses ${ }^{55,111}$. Therefore, I was interested to gain an understanding of the composition of MLN DC populations at steady-state. To do this, APC were isolated from the MLN of naïve mice and analysed for expression of cell surface markers by flow cytometry. On average, DC made up 1.5\% of lymph node resident cells with $0.5 \%$ of DC showing a CD11c + MHCII $^{\text {hi }}$ phenotype and $1 \%$ a CD11c $+\mathrm{MHCII}^{\mathrm{int}}$ phenotype. Each of these major DC populations could be could be further divided into four subsets based on cell surface expression of CD103 and CD11b. I found CD103+ CD11b+ and CD103+ CD11b- DC to be enriched in the CD11c+ MHCII ${ }^{\text {hi }}$ fraction and CD103CD11b+ and CD103- CD11b- DC to be enriched in the CD11c+ MHCII ${ }^{\text {int }}$ fraction (Fig. 3.7). The full gating strategy can be found in Appendix II. Furthermore, I found CD11c+ $\mathrm{MHCII}^{\text {hi }}$ DC to show elevated expression of the co-stimulatory molecule CD86 compared their CD11c+ MHCII ${ }^{\text {int }}$ counterparts (Fig. 3.8, A). In addition, there was modest expression of TIM-4 (Fig. 3.8, B) and little to no expression of the $\mathrm{M} \varphi$ markers F4/80, $\mathrm{CX}_{3} \mathrm{CR} 1$ and CD64 on any of these DC subsets in the MLN (Fig. 3.8, C-E). In summary, I found two distinct populations of DC in the MLN at steady-state which can be identified by their differential expression of CD103, MHCII and CD86. 
A
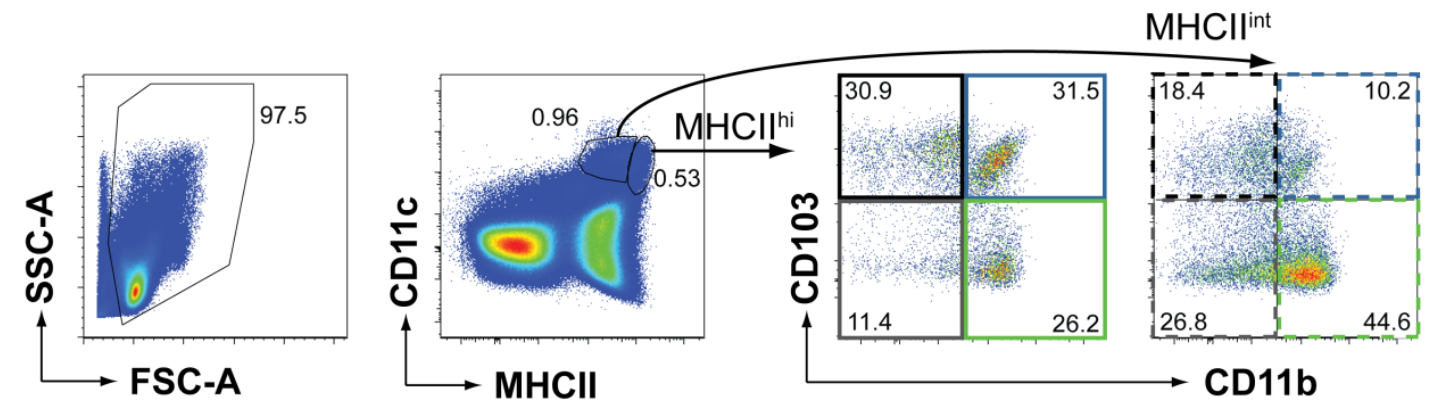

B

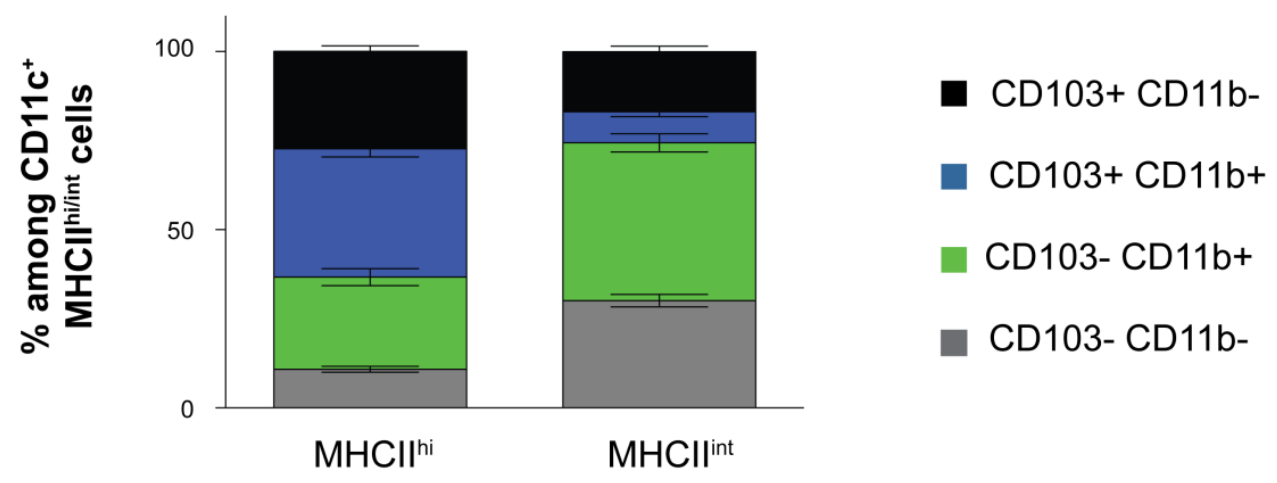

\section{Figure 3.7: DC subsets in the MLN}

Cells isolated from the MLN of naïve mice were stained and analysed by flow cytometry. DC were identified by CD11c and MHCII staining. MHCII hi and MHCII int DC were divided into subsets based on cell surface expression of CD103 and CD11b. Frequencies of DC subsets are shown on FACS dot plot (A) and bar graph (B). Graph shows mean \pm SEM of four independent experiments, each with 3 mice per experimental group. 


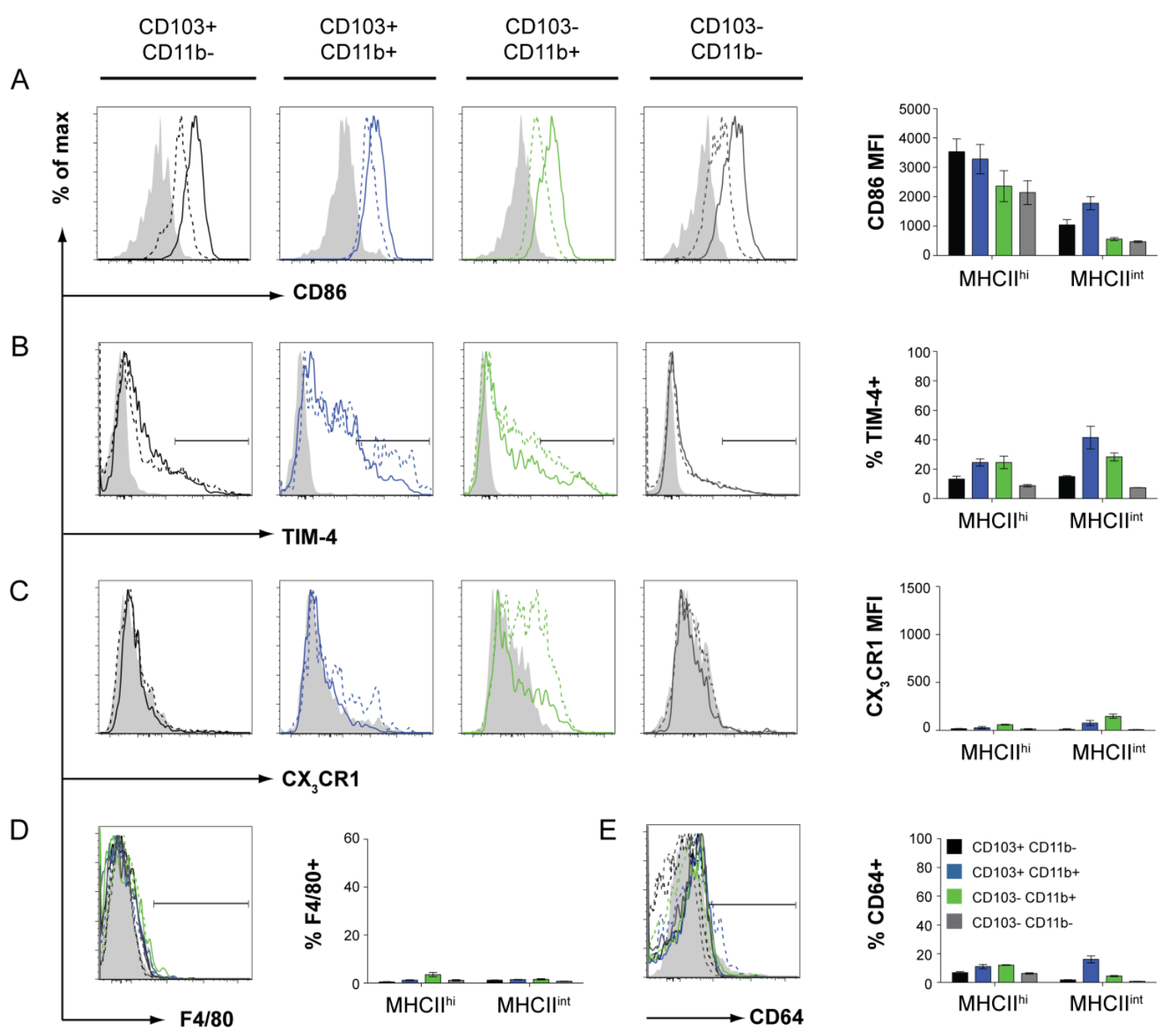

Figure 3.8: Cell surface markers on DC in the MLN

DC isolated from the MLN of naïve mice were assessed for cell surface markers by flow cytometry. CD86 (A), TIM-4 (B), CX 3 CR1 (C), F4/80 (D) and CD64 (E) staining is shown on histograms for $\mathrm{MHCII}^{\text {hi }}$ (solid line) and MHCII int (dashed line) DC subsets compared to FMO or isotype controls (shaded). Bar graphs show MFI or the frequency of positive cells within each DC subset. Graphs show mean \pm SEM of two to four independent experiments. 


\subsection{6. $\mathrm{MHCII}^{\mathrm{hi}}$ DC from the MLN have a high frequency of cells expressing active RALDH}

At steady-state, naïve T cells in the MLN are directed toward Treg differentiation, in part, through DC production of TGF $\beta$ and RA ${ }^{57,58}$. Examination of RALDH activity in each DC subset from the MLN using the flow cytometry ALDEFLUOR ${ }^{\text {TM }}$ assay showed the $\mathrm{CD} 11 \mathrm{c}+\mathrm{MHCII}^{\mathrm{hi}} \mathrm{DC}$ to have a higher frequency of RALDH+ cells compared to CD11c+ $\mathrm{MHCII}^{\text {int }}$ DC (Fig. 3.9, A). Among the MHCII ${ }^{\text {hi }}$ population, CD103+ CD11b+ and CD103+ CD11b- DC had a significantly higher frequency of cells with RALDH activity compared to CD103- DC subsets. Little to no RALDH activity was detected in MHCII ${ }^{\text {int }}$ populations, apart from a small population of $\mathrm{MHCII}^{\text {int }} \mathrm{CD} 103+\mathrm{CD} 11 \mathrm{~b}-\mathrm{DC}$, which appeared to have high RALDH activity (Fig. 3.9, B-C). This data shows CD11c+ MHCII ${ }^{\text {hi }}$ CD103+ DC to be the predominant source of cells with RALDH activity at steady-state in the MLN. 
A

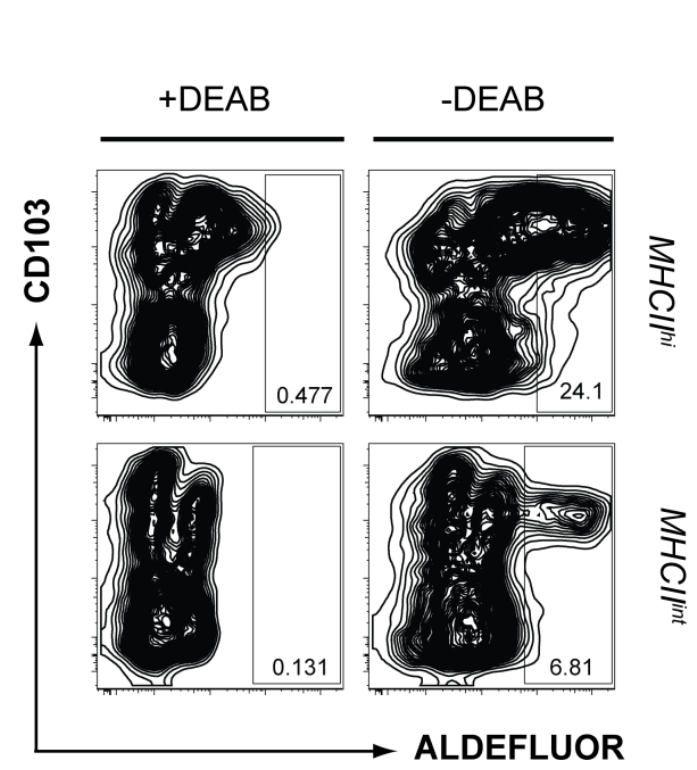

B

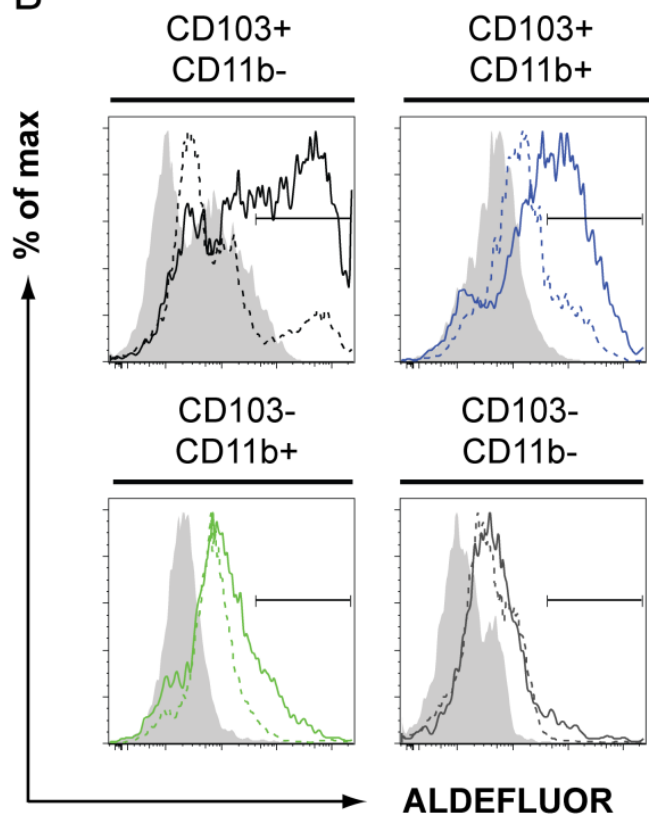

C

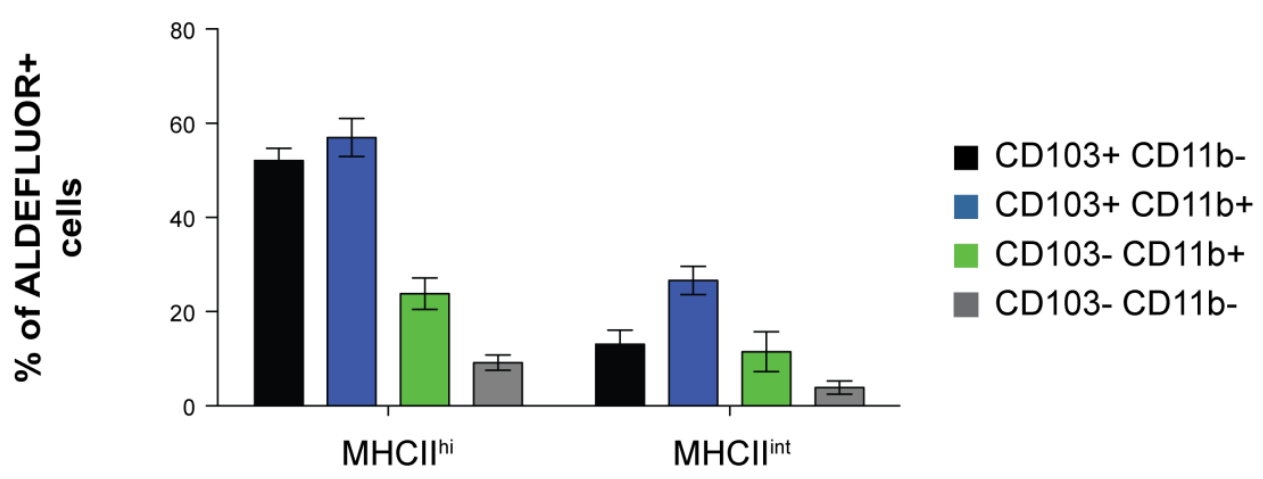

Figure 3.9: CD103+ DC in the MLN have active RALDH at steady-state

Cells isolated from the MLN of naïve mice were assessed for RALDH expression using an ALDEFLUOR staining kit. (A) Contour flow plots show ALDEFLUOR staining among CD11c+ MHCII $^{\text {hi }}$ and CD11c+ MHCII int cells compared to cells treated with RALDH inhibitor diethylaminobenzaldehyde (DEAB). B) Histograms show ALDEFLUOR staining for MHCII hi (solid line) and MHCII int (dashed line) DC subsets compared to DEAB treated control cells (shaded). C) Bar graph shows frequency of ALDEFLUOR+ cells within each DC subset. Graph shows mean \pm SEM. $n=3$. Data is representative of four independent experiments. 


\subsection{Discussion}

Intestinal APC drive tolerogenic responses at steady-state in order to maintain homeostasis. However, the distinction between DC and $M \varphi$ populations and their functions under homeostatic conditions remains ambiguous. Experiments in this Chapter show a novel method of characterising CD11c+ MHCII+ cells from the LP and MLN at steady-state. I found cell surface expression of CD103 and TIM-4 could identify three distinct subsets of CD11b + APC in the LP. TIM-4 expression by CD103- CD11b + cells correlated with cells of a $\mathrm{M} \varphi$ phenotype capable of antigen uptake in vivo whereas CD103+CD11b+ and CD103+ CD11b- DC showed superior activity of the enzyme RALDH, required for Treg induction, in both the LP and MLN. Therefore, I propose this is a robust strategy for identifying subsets of intestinal APC at steady-state and may be useful for future study.

\subsubsection{LP CD11b+ APC subsets at steady-state}

At steady-state, I found I could classify CD11b+ APC from the jejunal LP into three distinct populations based on cell surface expression of CD103 and TIM-4. Specifically these populations were: CD103+ TIM-4-, CD103- TIM-4+ and CD103- TIM-4-. Intestinal $\mathrm{CD} 11 \mathrm{~b}+\mathrm{APC}$ have been divided into two distinct populations, namely, CD103+ DC and $\mathrm{CX}_{3} \mathrm{CR} 1+\mathrm{M} \varphi$. Therefore, I hypothesised that CD103+ TIM-4- cells were phenotypically similar to migratory CD103+ DC that drive Treg differentiation at steady-state, whereas, CD103- TIM-4+ and CD103- TIM-4- were phenotypically similar to $\mathrm{CX}_{3} \mathrm{CR} 1+\mathrm{M} \varphi$ responsible for luminal antigen uptake.

In support of this hypothesis I found CD103+ TIM-4- cells to express RALDH, an enzyme associated with CD103+ DC that promote Treg differentiation ${ }^{57,58}$. Furthermore, CD103+ TIM-4- cells that lacked expression of the $\mathrm{M} \varphi$ markers $\mathrm{CX}_{3} \mathrm{CR} 1, \mathrm{~F} 4 / 80$ and $\mathrm{CD} 64$, were unable to take up antigen in vivo and had high expression of the DC marker CD11c. Thus, this data suggests CD103+ TIM-4- cells have a phenotype consistent with that of intestinal DC. In contrast, I found CD103- TIM-4+ cells expressed CX 3 CR1, F4/80 and CD64. 
CD103- TIM-4+ cells showed decreased CD11c expression compared to CD103+ TIM-4DC and lacked activity of RALDH. CD103- CD11b+ cells, which include CD103- TIM-4+ cells, showed significantly higher expression of activation markers MHCII and CD86 compared to other APC subsets. $\mathrm{CX}_{3} \mathrm{CR} 1+\mathrm{M} \varphi$ have previously been shown to localise to the epithelial barrier, allowing for increased exposure to microbial products ${ }^{75}$, which may explain the increased basal expression of these activation markers. Furthermore, following administration of fluorescent OVA antigen, I found CD103- TIM-4+ cells to be highly efficient at antigen uptake. Therefore, this data indicates that CD103- TIM-4+ cells are phenotypically and functionally similar to intestinal resident $\mathrm{M} \varphi$.

In contrast to my hypothesis, I found the CD103- TIM-4- subset to be a heterogeneous population that likely consists of a mixture of DC and $\mathrm{M} \varphi$. Phenotypic analysis of this population found expression of $\mathrm{CX}_{3} \mathrm{CR} 1$ and F4/80 was significantly lower than CD103TIM-4+ M $\varphi$. In addition, approximately 40\% of CD103- TIM-4- cells were CD64- which suggests they are not of monocytic lineage. Interestingly, I found 30-35\% CD103- TIM-4cells to be RALDH+. Together these observations indicate that up to $40 \%$ of the CD103TIM-4- population may have phenotypic features similar to that of intestinal DC. This interpretation is supported by recent studies which show that a proportion of $\mathrm{CX}_{3} \mathrm{CR} 1+$ cells express DC specific protein zBTB46 ${ }^{103}$. However, further investigation is required to confirm the lineage of the CD103- TIM-4- CD64- cells. Furthermore, a small population of $\mathrm{CX}_{3} \mathrm{CR} 1^{\text {int }}$ cells have been reported in the LP at steady-state. These cells are thought to be more similar to $\mathrm{DC}$ than $\mathrm{M} \varphi$ as they expand in response to Flt3 $\mathrm{L}$ and are able to drive $\mathrm{T}$ cell proliferation ${ }^{42}$. However, like $\mathrm{M} \varphi$, they originate from monocytic precursors and thus, these DC express CD64, a marker which has been previously used to identify intestinal $\mathrm{M} \varphi{ }^{113}$. Due to the lower expression of $\mathrm{CX}_{3} \mathrm{CR} 1$ among CD103- TIM-4- cells, it is possible that a population of moDC also reside within this gate. Finally, I found a significantly lower frequency of CD103- TIM-4- cells took up antigen in vivo compared to CD103- TIM-4+ cells. This would suggest that the LP resident M $\varphi$ that lack expression of TIM-4 may be functionally distinct from CD103- TIM-4+ M $\varphi$. 
In summary, by using CD103 and TIM-4 expression to characterise intestinal CD11b+ APC subsets I highlighted the presence of three distinct populations of APC in the LP at steadystate. CD103+ TIM-4- DC are phenotypically similar to the tolerogenic CD103+ DC reported in the literature. In addition, CD103- TIM-4+ M $\varphi$ share phenotypic and functional similarities to $\mathrm{CX}_{3} \mathrm{CR} 1+\mathrm{M} \varphi$. However, the CD103- TIM-4- cells made up a heterogeneous population of DC and $\mathrm{M} \varphi$, which have previously not been characterised. These DC and $\mathrm{M} \varphi$ may play different roles to earlier described DC and $M \varphi$ in maintaining tolerance at steadystate and may provide an interesting avenue for further study.

\subsubsection{LP CD11b- APC subsets at steady-state}

In addition to $\mathrm{CD} 11 \mathrm{~b}+\mathrm{APC}$, I found that the jejunal LP contains small numbers of CD103+ CD11b- and CD103- CD11b-cells, however, the frequency of these cells varied significantly between mice. CD103+ CD11b- cells lacked expression of the $\mathrm{M} \varphi$ markers $\mathrm{CX}_{3} \mathrm{CR} 1, \mathrm{CD} 64$ and $\mathrm{F} 4 / 80$ but had some RALDH activity suggesting they are phenotypically similar to DC. CD103-CD11b- cells showed some expression of the $\mathrm{M} \varphi$ markers F4/80, CD64 and TIM-4 but also showed some RALDH activity suggesting this population likely consisted of a mixture of $\mathrm{DC}$ and $\mathrm{M} \varphi$.

It has been proposed that CD103+ CD11b- cells, which are found in high numbers in lymphoid organs ${ }^{104}$, can be found in the LP preparations if Peyer's patches are not completely removed from the jejunal tissue ${ }^{101}$. Furthermore, the frequency of CD103+ CD11b-cells is thought to be dependent on the section of intestine, with higher frequencies found closer to the stomach ${ }^{102}$. Therefore, as I could not accurately account for the origin of CD103+ CD11b- cells in my preparations, I excluded this population from further analysis.

\subsubsection{MLN DC subsets at steady-state}

Analysis of MLN DC showed two distinct populations based on the level of MHCII expression. These two populations could be further divided into four subsets based on expression of CD103 and CD11b. In other lymph nodes, high expression of MHCII has 
been correlated to migratory DC populations and intermediate expression with lymph node resident populations ${ }^{44}$. However, this strategy has not been commonly utilised in the MLN. Therefore, I hypothesised that CD11c+ MHCII ${ }^{\text {hi }}$ cells were migratory DC that had migrated to the MLN from the intestine, whereas, $\mathrm{CD} 11 \mathrm{c}+\mathrm{MHCII}^{\mathrm{int}}$ cells were lymph node resident DC.

In support of this hypothesis, I found the $\mathrm{CD} 11 \mathrm{c}+\mathrm{MHCII}{ }^{\mathrm{hi}}$ population to have upregulated activation marker CD86 compared to the $\mathrm{CD} 11 \mathrm{c}+\mathrm{MHCII}{ }^{\text {int }}$ population. In addition, the CD11c+ MHCII ${ }^{\text {hi }}$ population was enriched for CD103+ CD11b- and CD103+ CD11b+ DC and these populations were shown to have high RALDH activity. Previous reports have shown CD103+ DC migrate from the intestine to the MLN to drive Treg differentiation at steady-state ${ }^{55,111}$. In contrast, the CD11c+ MHCII ${ }^{\text {int }}$ population was enriched for CD103CD11b+ and CD103- CD11b- cells that showed little to no RALDH activity. In the LP, a large proportion of $\mathrm{CD} 103-\mathrm{CD} 11 \mathrm{~b}+$ cells express the $\mathrm{M} \varphi$ markers $\mathrm{CX}_{3} \mathrm{CR} 1, \mathrm{~F} 4 / 80$ and CD64. There was little to no expression of these markers on any DC populations in the MLN which suggests LP M $\varphi$ do not migrate to the MLN. However, I found variable expression of TIM-4 among all MLN DC subsets. While TIM-4 has been associated with $\mathrm{M} \varphi$, others have shown that DC can upregulate TIM-4 upon activation ${ }^{148}$. In contrast, the $\mathrm{CD} 11 \mathrm{c}+\mathrm{MHCII}^{\mathrm{hi}}$ population in the MLN appears to be enriched for RALDH CD103+ DC which may have migrated from the LP. These results suggest that the CD11c+ MHCII ${ }^{\text {hi }}$ cells contain APC that closely resemble cells from the LP and thus are likely to consist of the migratory DC population. Therefore, for my future studies I focused on CD11c $+\mathrm{MHCII}^{\mathrm{hi}}$ cells. 


\subsection{Conclusions}

The population of intestinal APC is diverse, complex and unlike that of other tissues. Therefore, the understanding of the composition of steady-state APC subsets and their functions is incomplete. In this chapter, I show the characterisation of LP APC by assessing expression CD103 and TIM-4 and of MLN DC by assessing level of MHCII expression. CD103+ TIM-4- DC express high levels of RALDH and can be found in the LP and among CD11c+ MHCII ${ }^{\text {hi }}$ cells in the MLN. On the other hand, CD103- TIM-4+ cells are found in the LP and possess phenotypic and functional characteristics of $\mathrm{M} \varphi$. These include the expression of $\mathrm{CX}_{3} \mathrm{CR} 1, \mathrm{CD} 64$ and F4/80 and a high efficiency of antigen uptake in vivo. Finally, CD103- TIM-4- cells appear to comprise of a heterogeneous population that most likely consists of cells of the DC and $\mathrm{M} \varphi$ lineage. From data presented in this chapter, I show that APC subsets in the intestine are highly heterogeneous at steady-state. I propose that CD103 and TIM-4 expression in the LP and MHCII expression in the MLN will be useful in future studies to help identify functionally distinct DC and $\mathrm{M} \varphi$ populations in the intestine. This question will be examined later in this thesis (Chapter 5). 


\section{Chapter 4: Phenotypic characterisation of intestinal APC subsets following oral administration of a mucosal adjuvant}




\subsection{Introduction}

Intestinal APC are specialised to drive tolerogenic responses at steady-state while maintaining the capacity to recognise and initiate effector responses to pathogenic microbes and helminths ${ }^{81}$. Although the mechanisms by which intestinal APC differentiate between innocuous and harmful antigens is currently unknown, mucosal adjuvants and inflammatory stimuli can alter intestinal APC phenotype and function. Changes in phenotype and subset composition may promote the initiation of immune responses in the intestine. Despite this, there is a lack of information on direct comparison between small intestinal APC populations at steady-state and following administration of a mucosal adjuvant. Study of APC following a mucosal adjuvant may provide insight into the processes that lead to the development of immunity in the intestine.

At steady-state, CD103+ CD11b+ DC play a critical role in driving tolerance by promoting differentiation of Tregs in the LP and MLN ${ }^{57,58}$. However, CD103+ CD11b+ DC also play an important role in the direction of effector Th1/Th17 responses ${ }^{81,102}$ and IL-22/23 responses to TLR5 ligands ${ }^{174}$. Danger signals promote the accumulation of CD103+ $\mathrm{CD} 11 \mathrm{~b}+\mathrm{DC}$ in the $\mathrm{LP}_{\text {and }} \mathrm{MLN}{ }^{86,175}$ and promote phenotypic and functional changes within this population. For example, Salmonella challenge has been shown to induce the mobilisation of CD103+ CD11b+ DC to the epithelial barrier to capture bacteria in a chemokine and TLR-dependent fashion ${ }^{70,}{ }^{72}$. In addition, CD103+ CD11b+ DC isolated from the MLN following the initiation of effector responses, have significantly lower expression of the genes associated with production of tolerogenic chemical mediators TGF $\beta$ and RA and increased expression of pro-inflammatory genes such as tbet and il-12p35 ${ }^{175}$. However, it has yet to be determined whether there are distinct subsets within the CD103+ $\mathrm{CD} 11 \mathrm{~b}+$ population that preferentially promote different immune responses, or whether environmental cues act on the population as a whole to promote immunity.

Under homeostatic conditions, monocytic precursors differentiate into tolerogenic, IL-10 producing $\mathrm{CX}_{3} \mathrm{CR} 1^{\text {hi }} \mathrm{M} \varphi$ and small numbers of $\mathrm{CX}_{3} \mathrm{CR} 1^{\text {int }} \mathrm{DC}{ }^{42,134}$. During inflammation, 
the composition of the monocyte-derived compartment changes and $\mathrm{CX}_{3} \mathrm{CR}^{\text {int }} \mathrm{DC}$ increase significantly in frequency in the LP. $\mathrm{CX}_{3} \mathrm{CR} 1^{\text {int }} \mathrm{DC}$ are thought to promote effector $\mathrm{T}$ cell responses in the intestine and have a pro-inflammatory genetic signature ${ }^{99,117,134,135} 114$. These DC, when isolated from inflamed colons, have been shown to drive CD4 $\mathrm{T}$ cell proliferation ${ }^{99}$ and IFN $\gamma$ production ${ }^{114}$ in vitro. Ablation of the $\mathrm{CX}_{3} \mathrm{CR} 1{ }^{\text {int }}$ population with an anti-CCR2 antibody ameliorated DSS induced colitis, implicating these cells as the major drivers of colonic inflammation ${ }^{99}$. However, the majority of study into intestinal moDC has been focused on the colon and it is not understood whether moDC play a role in driving immunity in the small intestine.

While there is some understanding about the roles of APC subsets in the promotion of immunity in the colon, the roles of APC from the small intestine are less well defined. Cholera toxin (CT) is a potent mucosal adjuvant that drives immune responses to codelivered antigen in the small intestine ${ }^{176}$. In addition, CT disrupts the epithelial barrier allowing for entry of commensal bacteria into the LP ${ }^{177}$. Prolonged interaction between commensals and immune cells is known to cause chronic intestinal inflammation ${ }^{178}$. I used oral administration of CT to study the effects of a mucosal adjuvant on the composition and phenotype of APC subsets from the small intestine in vivo. From these studies, I identified a novel phenotype of DC, characterised by co-expression of CD103 and TIM-4, which appear transiently in the LP and MLN following oral administration of CT. I propose that LPresident CD103+ DC upregulate TIM-4 upon exposure to CT and subsequently migrate to the lymph node. In the lymph node, CD103+ TIM-4+ DC may influence T cell responses and thus contribute to the initiation of immunity in the intestine. 


\subsection{Aims}

APC are important in regulating intestinal immune responses. Adjuvants and other stimulatory agents can alter the phenotype and function of APC and thus influence T cell responses. For these reasons, I was interested to assess the phenotype and subset composition of intestinal APC after oral administration of the mucosal adjuvant CT. This chapter summarises my characterisation of intestinal APC subsets following oral administration of CT.

Specifically my aims were:

1. To determine whether APC subset compositions in the LP and MLN are altered following oral administration of CT.

2. To assess the phenotype and origin of the populations that are altered following oral administration of CT.

3. To assess the roles of commensal bacteria and TLR4 signalling in any observed changes to intestinal APC subsets following oral administration of CT. 


\subsection{Results}

\subsubsection{Oral administration of cholera toxin changes the composition of APC subsets in the LP and MLN}

In Chapter 3, I showed that steady-state CD11b+ APC can be divided into three distinct populations based on expression of CD103 and TIM-4: CD103+ TIM-4- DC, CD103- TIM4+ M $\varphi$ and a heterogeneous population of CD103- TIM-4- cells. Using this method, I wished to compare the composition of APC subsets after CT treatment and in the steadystate. To address this, mice were gavage fed the mucosal adjuvant CT with OVA antigen $(\mathrm{OVA}+\mathrm{CT})$. Cells were isolated from the LP and MLN 17 hours later and assessed for expression of cell surface markers.

I found that $\mathrm{CT}$ treatment did not result in significant changes to the frequency of total number of CD11c+ MHCII+ APC in the LP. Furthermore, the frequency of APC subsets as determined by CD103 and CD11b staining (CD103+ CD11b-, CD103+ CD11b+, CD103- CD11b+ and CD103- CD11b-) did not change between steady-state and CT treatment (Fig. 4.1, A). I observed approximately 50\% of CD103+ CD11b+ cells expressed the cell surface marker TIM-4. Therefore, CD11b+ APC from OVA+CT treated mice could be divided into four distinct populations due to the appearance of CD103+ TIM-4+ cells in the LP (Fig. 4.1, A-B). In addition to examining expression of CD11b, CD103 and TIM-4, I also assessed the expression of the activation marker CD86 on LP APC subsets. I found that OVA + CT treatment resulted in a significant upregulation of CD86 on CD103+ CD11b+ and CD103+ CD11b- LP APC (Fig. 4.1, C).

Similar to the LP, the composition of MLN CD11c+ MHCII ${ }^{\text {hi }}$ DC subsets did not change between naïve and OVA+CT treated mice. However, the proportion of TIM-4+ cells among CD103+ CD11b+ DC increased on average from 20\% to 50\% following OVA+CT (Fig. 4.2, A-B). Although not significant in all experiments carried out, CD86 expression 
tended to be higher on CD103+ DC after CT treatment compared to steady-state (Fig. 4.2, C). 
A
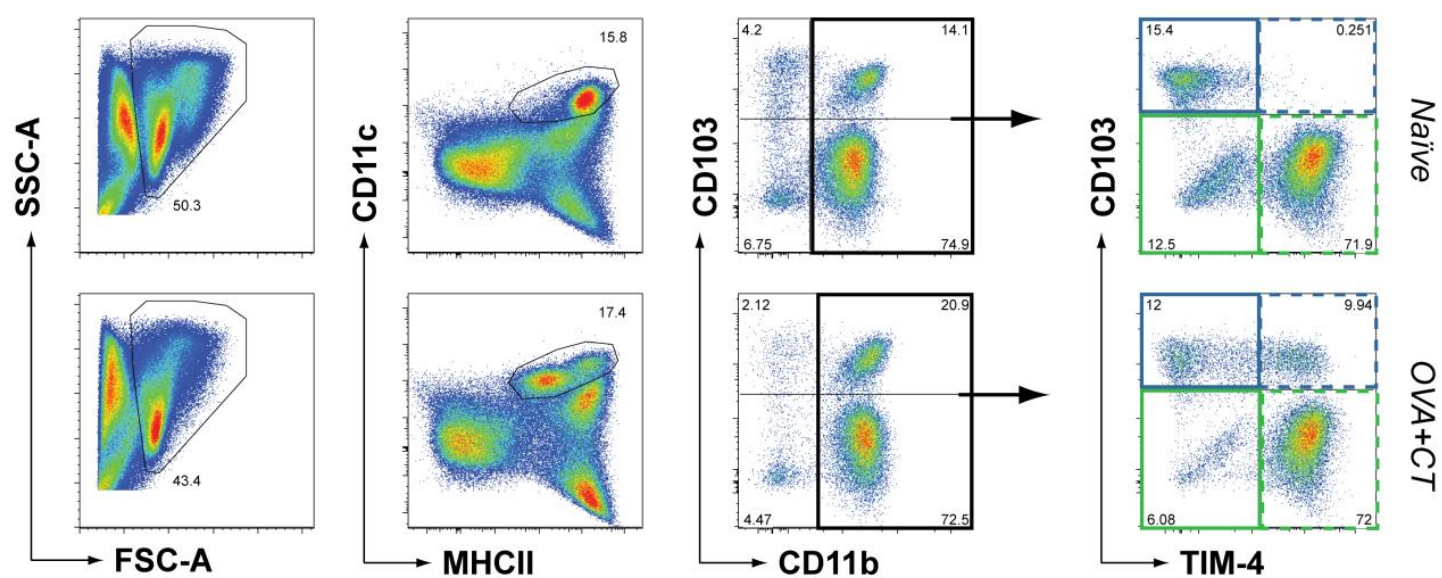

B

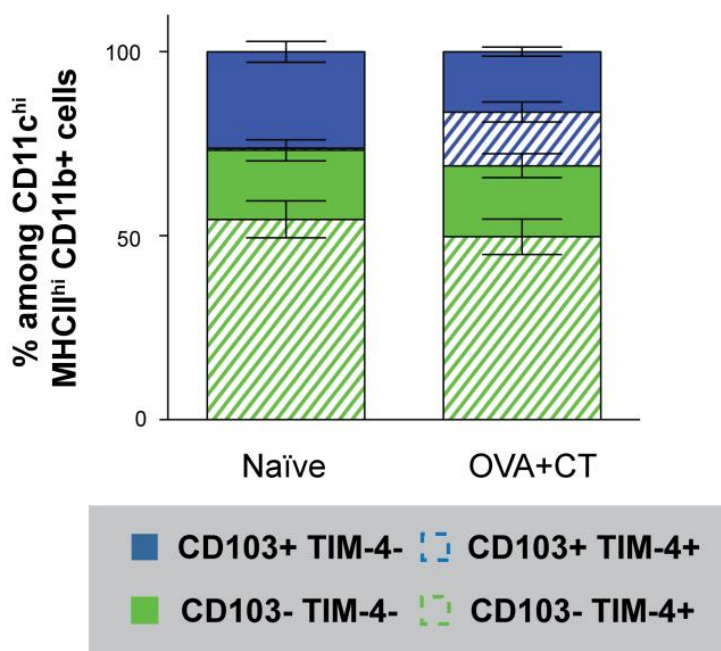

C

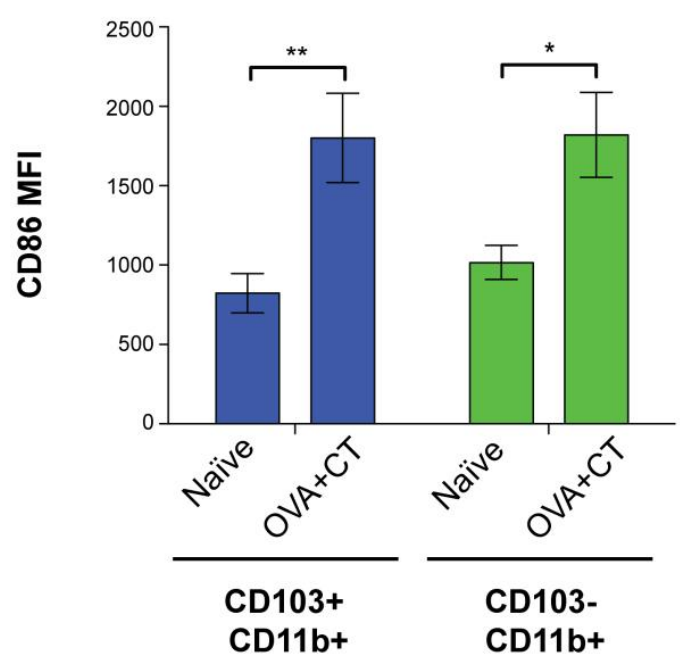

Figure 4.1: CT induces the appearance of a novel phenotype of APC in the LP

LP cells isolated from naïve or OVA+CT treated mice were stained and analysed by flow cytometry. APC were identified by CD11c and MHCII staining and then divided into subsets based on cell surface expression of CD103, TIM-4 and CD11b. Gating strategy to classify APC subsets from the jejunal LP are shown on FACS dot plots (A). Bar graphs show frequency of each APC subset from the LP (B) and CD86 MFI on CD11b+ APC (C). Graph shows combined mean \pm SEM of four independent experiments, each of which included 3 mice per experimental group. Statistical significance was determined by Student's unpaired t-test. $* \mathrm{p}<0.05, * * 0.001<\mathrm{p}<0.05$ 
A
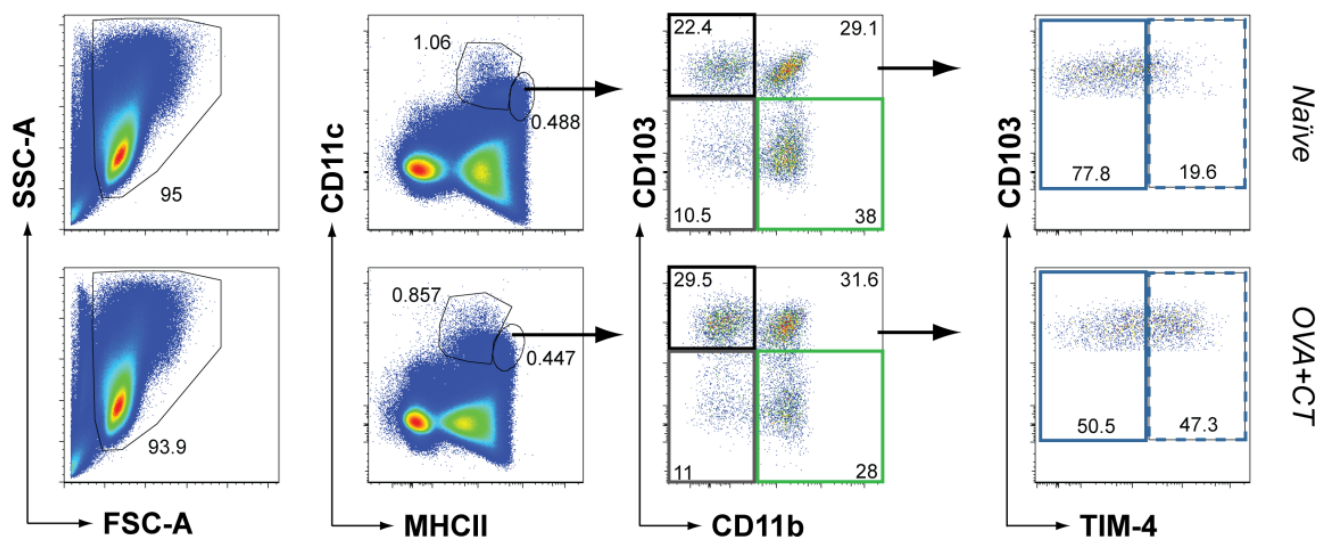

B

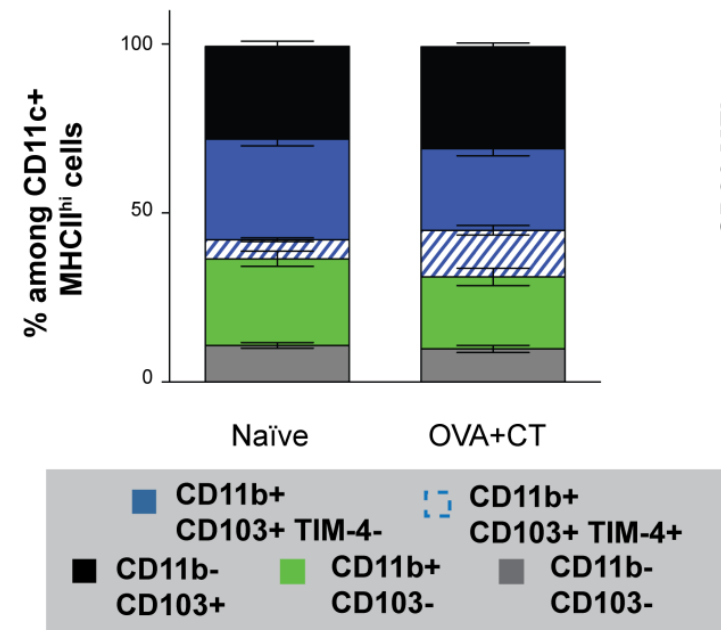

C

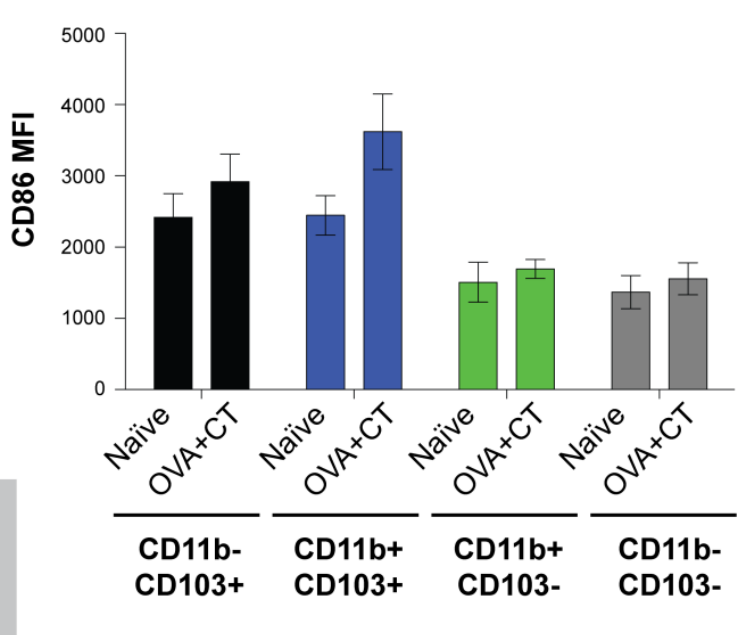

Figure 4.2: CT increases the frequency of CD103+ CD11b+ TIM-4+ APC in the MLN

MLN cells isolated from naïve or OVA+CT treated mice were stained and analysed by flow cytometry. APC were identified by CD11c and MHCII staining and then divided into subsets based on cell surface expression of CD103, TIM-4 and CD11b. Gating strategy to classify APC subsets from the MLN are shown on FACS dot plots (A). Bar graphs show frequency of each APC subset from the MLN (B) and CD86 MFI on each APC subset (C). Graph shows combined mean \pm SEM of four independent experiments, each of which included 3 mice per experimental group. 


\subsubsection{CT is sufficient to induce the appearance of CD103+ TIM-4+ DC in the intestine}

Oral administration of $\mathrm{CT}$ in combination with OVA protein leads to an increase in the frequency of CD103+ TIM-4+ cells in the LP and MLN. Although not significant in all experiments, similar results can be observed in mice co-administered BSA protein and CT. However, I found administration of CT alone in PBS did not alter APC subset compositions from steady-state. Thus, I hypothesised the activity of CT was being compromised by the acidity of the stomach and co-administration with a large bolus of protein such as OVA or BSA was protecting CT from degradation. To test this hypothesis, CT was administered in bicarbonate buffer ( $\mathrm{pH}$ 9.6) to increase the $\mathrm{pH}$ of the stomach. This experiment showed CT induced a similar increase in the frequency of CD103+ TIM-4+ cells compared to CT combined with OVA or BSA (Fig. 4.3). This data demonstrates that CT is responsible for the change in APC subset composition I observe following oral administration of OVA+CT.

\subsubsection{CD103+ TIM-4+ cells appear transiently in the intestine following OVA+CT treatment}

As I could detect CD103+ TIM-4+ DC at increased frequencies in both the LP and MLN, I was interested to determine the kinetics of CD103+ TIM-4+ cells in order to gain a better understanding of their origin and migratory capacity. To do this, cells were isolated from the LP and MLN at different time points following oral administration of OVA+CT. I found that CD103+ TIM-4+ cells begun to appear in the LP from 8 hours post treatment, peaked in frequency at 17 hours and decreased in frequency from 24 hours (Fig. 4.4, A). In the MLN, CD103+ TIM-4+ cells could be detected from 12 hours post treatment and returned to baseline by 48 hours (Fig. 4.4, B). This data shows CD103+ TIM-4+ cells initially increase in frequency in the LP after CT treatment and can be detected in the MLN 4 hours later. The appearance of CD103+ TIM-4+ cells appears to be transient with the frequency of APC subsets from CT treated mice returning to steady-state by 48 hours in the MLN. 
A

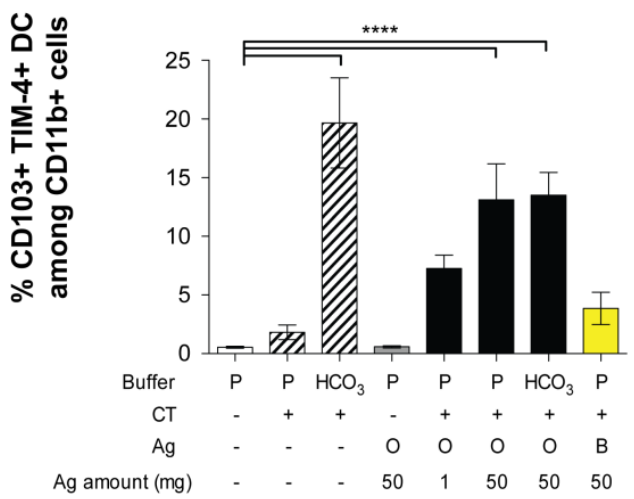

B

Mesenteric lymph node

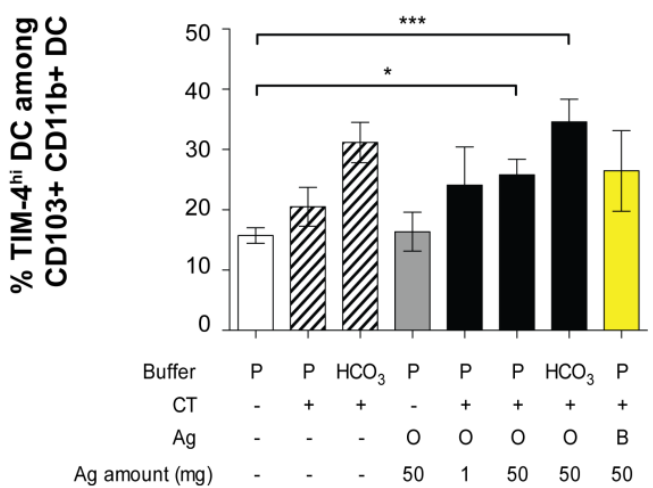

Figure 4.3: CT induces the appearance of CD103+ TIM-4+ APC in the LP and MLN

Cells were isolated from mice treated with CT, OVA $(\mathrm{O})$, OVA+CT or BSA $(\mathrm{B})+\mathrm{CT}$ in PBS $(\mathrm{P})$ or bicarbonate buffer $\left(\mathrm{HCO}_{3}\right)$ by oral gavage. Frequency of CD103+ TIM-4+ among $\mathrm{CD} 11 \mathrm{~b}+\mathrm{APC}$ from the LP (A) and TIM-4+ among CD103+ CD11b+ APC from the MLN (B) is shown on bar graphs. Graphs shown mean \pm SEM. Graphs were prepared by combining data from three independent experiments. Statistical significance was determined by One-Way ANOVA with Bonferroni post-test. $* \mathrm{p}<0.05, * * * 0.0001<\mathrm{p}<0.001, * * * * \mathrm{p}<0.0001$

A

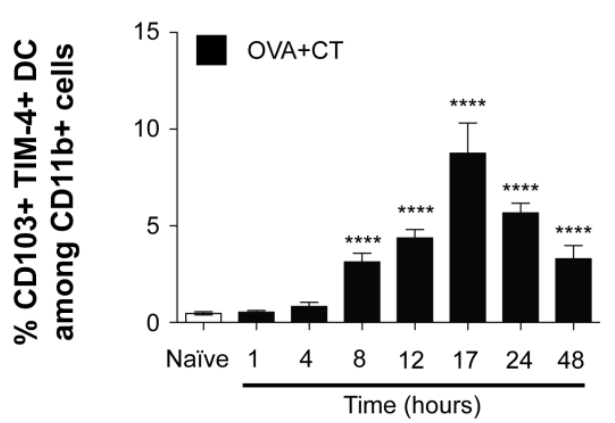

B

Mesenteric lymph node

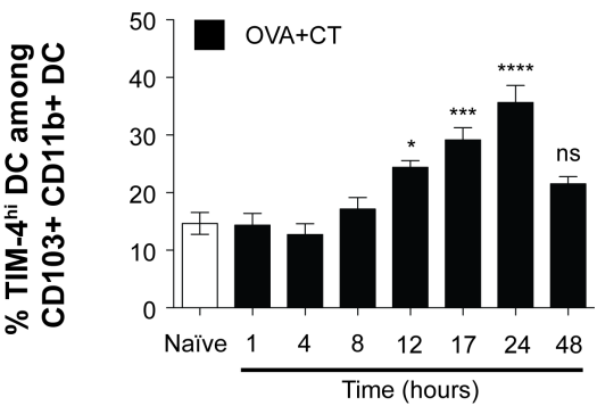

Figure 4.4: CD103+ TIM-4+ APC appear transiently in the LP and MLN following CT administration

Cells were isolated from mice 1-48 hours following intragastric OVA+CT administration. Frequency of CD103+ TIM-4+ APC among total CD11b+ APC from the LP (A) and TIM-4+ among CD103+ CD11b+ APC from the MLN (B) was determined by flow cytometry for each time point. Graphs shown mean \pm SEM. Graphs were prepared by combining data from three independent experiments. Statistical significance was determined by One-Way ANOVA with Bonferroni post-test. $* \mathrm{p}<0.05, * * 0.001<\mathrm{p}<0.05, * * * 0.0001<\mathrm{p}<0.001, * * * * \mathrm{p}<0.0001$ 


\subsection{4. $\mathrm{CT}$ reduces expression of $\mathrm{CX}_{3} \mathrm{CR} 1$ but not $\mathrm{F} 4 / 80$ or $\mathrm{CD} 64$ on CD103- APC}

As I could detect TIM-4 expression on CD103+ DC following CT treatment, I was interested to determine whether CT altered the expression of other cell surface markers on other APC subsets. Therefore, I compared cell surface expression of the lineage markers $\mathrm{CX}_{3} \mathrm{CR} 1, \mathrm{~F} 4 / 80$ and CD64 among LP APC subsets from naïve and OVA+CT treated mice. Interestingly, I found that the expression of $\mathrm{CX}_{3} \mathrm{CR} 1$ decreased following CT treatment on both CD103- TIM-4+ M $\varphi$ and CD103- TIM-4- cells (Fig. 4.5, A). However, there was no difference in the expression of F4/80 or CD64 between steady-state and CT treatment in any of the LP APC populations (Fig. 4.5, B-C).

\subsubsection{CD103+ TIM-4+ cells are phenotypically similar to CD103+ TIM-4- DC}

The increase in frequency of CD103+ TIM-4+ cells was the most significant difference I observed between steady-state and CT treatment. Therefore, I was interested to further characterise CD103+ TIM-4+ cells. To do this, I examined the expression of lineage markers on CD103+ TIM-4+ cells. From these studies, I found CD103+ TIM-4- DC and CD103+ TIM-4+ cells lacked expression of $\mathrm{M} \varphi$ markers $\mathrm{CX}_{3} \mathrm{CR} 1$ and F4/80, whereas these markers could be detected on the CD103- TIM-4+ M $\varphi$ population (Fig. 4.5, A-B). CD64 is a monocyte lineage marker that is found on both $\mathrm{M} \varphi$ and inflammatory DC populations ${ }^{50}$, 113. CD103+ TIM-4- DC and CD103+ TIM-4+ cells were found to express little to no CD64, whereas approximately $60 \%$ of CD103- TIM-4- cells and 95\% of CD103- TIM-4+ $\mathrm{M} \varphi$ express CD64 (Fig. 4.5, C).

In addition to assessing cell surface lineage markers, I compared the cellular morphology of CD103+ TIM-4+ cells to previously characterised intestinal CD103+ TIM-4- DC and CD103- TIM-4+ M $\varphi$ populations. I used flow cytometry to sort individual LP CD11c+ $\mathrm{MHCII}+$ populations from OVA+CT treated mice, and stained cells using DiffQuick to observe under the microscope. I found CD103+ TIM-4- cells to have the typical morphology of classical DC, with dendrite protrusions and an amorphous cytoplasm. Conversely, the CD103- TIM-4+ population had the typical morphology of $\mathrm{M} \varphi$, with many 
phagocytic vacuoles in the cytoplasm. CD103- TIM-4- cells tended to be a heterogeneous population, with a morphology not typical of DC or M $\varphi$. CD103+ TIM-4+ cells appeared round and had very few dendritic protrusions or cytoplasmic vacuoles (Fig. 4.6, A). Thus, the morphology of CD103+ TIM-4+ cells was observed to have features of both DC and $\mathrm{M} \varphi$. Importantly, flow cytometric analysis revealed CD103+ TIM-4+ cells to have forward and side scatter properties similar to those of CD103+ TIM-4- DC and not CD103- TIM4+ $\mathrm{M} \varphi$ (Fig. 4.6, B). In summary, data from phenotypic, morphological and flow cytometry studies suggest that CD103+ TIM-4+ cells that appear following CT treatment are more similar to CD103+ TIM-4- DC than CD103- TIM-4+ M $\varphi$.

\subsubsection{CT induces maturation of CD103+ TIM-4+ cells in the LP}

I showed that CT treatment induced the upregulation of the activation marker CD86 on CD11b+ APC from the LP (Fig. 4.1, C). Therefore, I was interested to assess whether CT preferentially induces maturation of specific subsets of APC in the intestine. I found the population of CD103+ TIM-4+ cells to have significantly elevated expression of CD86 compared to CD103+ TIM-4- DC and CD103- TIM-4+ M $\varphi$ populations (Fig. 4.5, D). Interestingly, CD103+ TIM-4- DC showed similar CD86 expression between steady-state and inflammatory conditions. Therefore, this data suggests CD103+ TIM-4+ cells are accounting for the increase in CD86 expression observed among CD103+ CD11b+ cells (Fig. 4.1., C). 


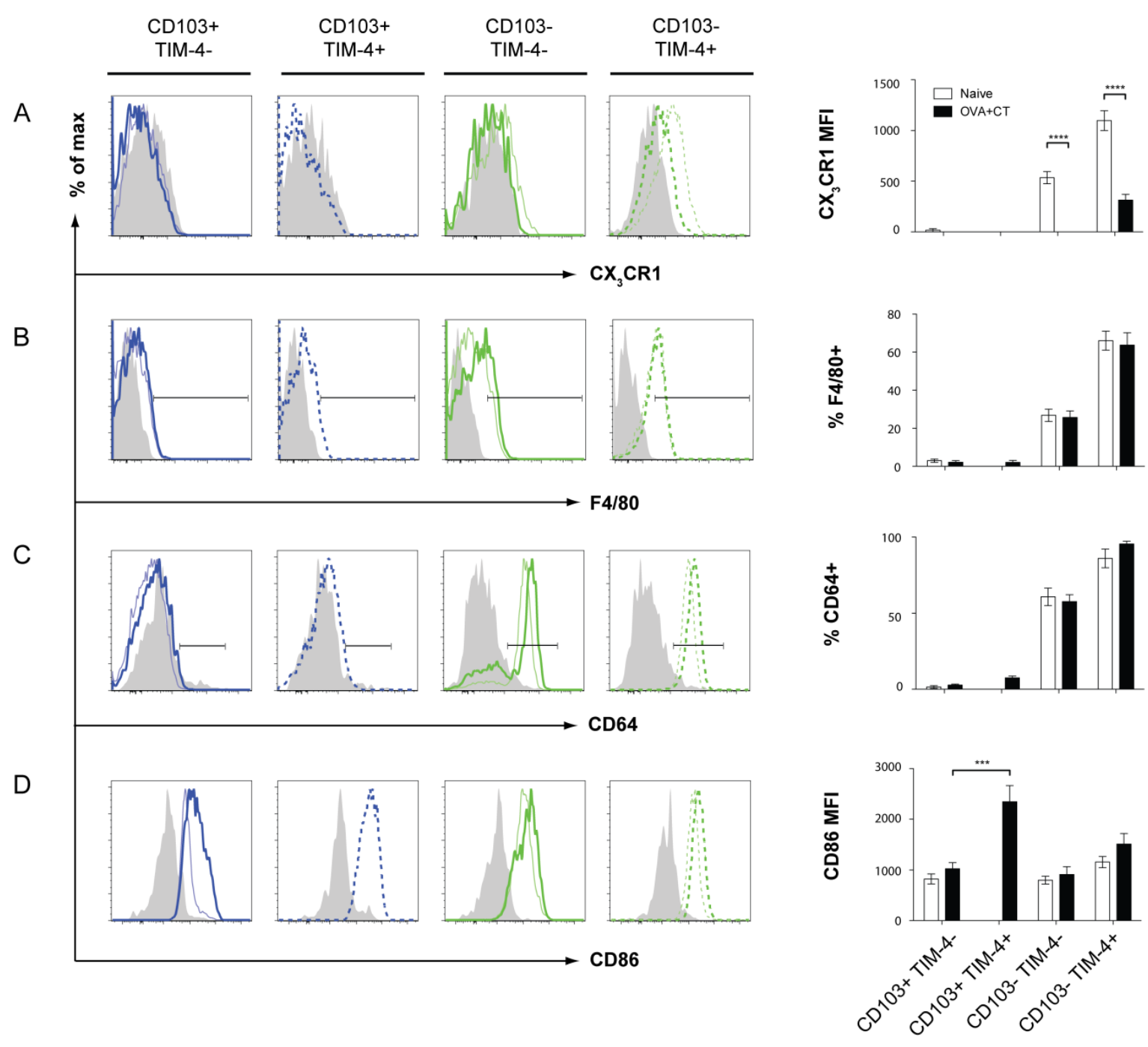

Figure 4.5: CD103+ TIM-4+ APC show increased activation and lack markers of M $\varphi$ lineage.

LP APC were isolated from naïve mice or 17 hours following intragastric OVA+CT. Cell surface expression of $\mathrm{CX}_{3} \mathrm{CR} 1$ (A), F4/80 (B), CD64 (C) and CD86 (D) is shown on histograms for each subset (bold coloured line) compared to naïve mice (coloured line) and FMO or isotype controls (shaded). MFI or percent positive among APC subset are shown on corresponding bar graphs. Graphs show combined mean \pm SEM of two to four independent experiments. Statistical significance was determined by One-Way ANOVA with Bonferroni post-test (A) or Student's unpaired t-test (D). ${ }^{* * *} 0.0001<\mathrm{p}<0.001,{ }^{* * * *} \mathrm{p}<0.0001$ 


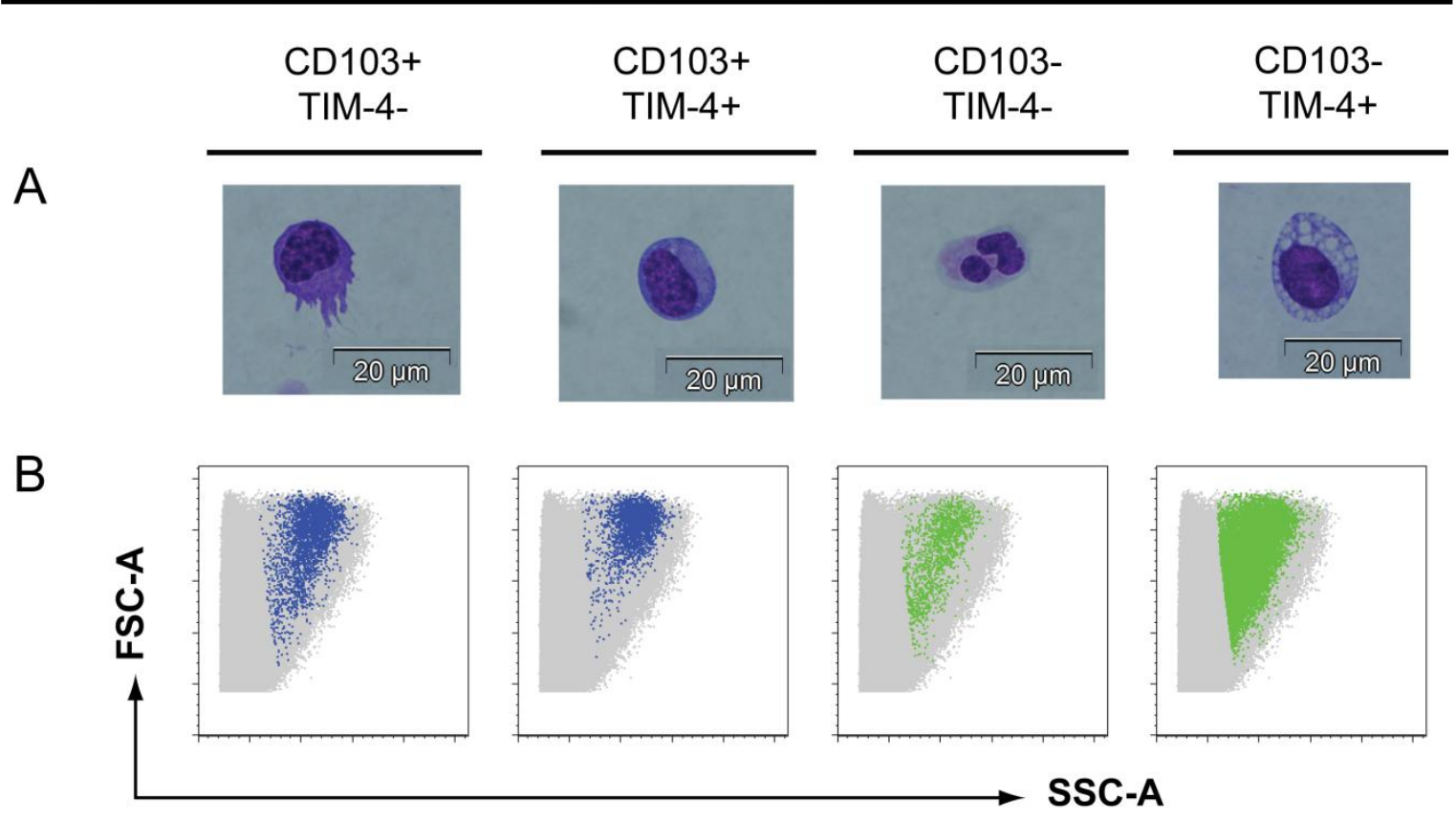

Figure 4.6: CD103+ TIM-4+ APC share morphological characteristics of CD103+ TIM-4- DC and CD103+ TIM-4+ M $\varphi$

LP APC were isolated 17 hours following intragastric OVA+CT. CD103+ TIM-4-, CD103+ TIM4+, CD103- TIM-4- and CD103- TIM-4+ cells were flow sorted and stained with DiffQuick (A).Dot plots show FSC and SSC characteristics for each APC population following oral OVA+CT (B).

\subsubsection{CD103+ TIM-4- and CD103+ TIM-4+ cells express active RALDH}

I was interested to determine expression of the enzyme RALDH among APC subsets from the LP. RALDH has been shown to be highly active among CD103+ DC from the intestine. I measured RALDH activity by flow cytometry using the ALDEFLUOR assay and found that among CD103+ TIM-4- and CD103+ TIM-4+ populations, a similar proportion (7090\%) of cells had detectable RALDH activity. In comparison, only 1-2\% of CD103- TIM4+ M $\varphi$ and 20-25\% of CD103- TIM-4- cells showed RALDH activity (Fig. 4.7, A-B). This data shows that CD103+ TIM-4+ cells have similar RALDH activity to intestinal CD103+ TIM-4- DC from CT treated mice. 


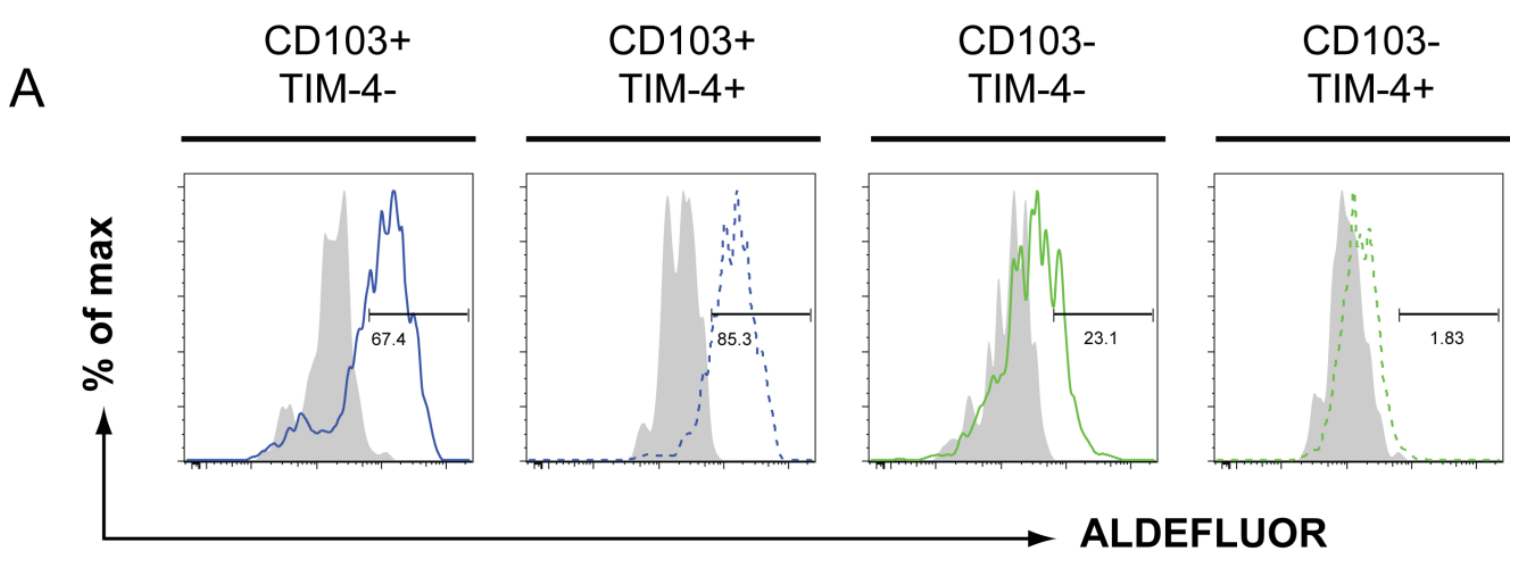

B

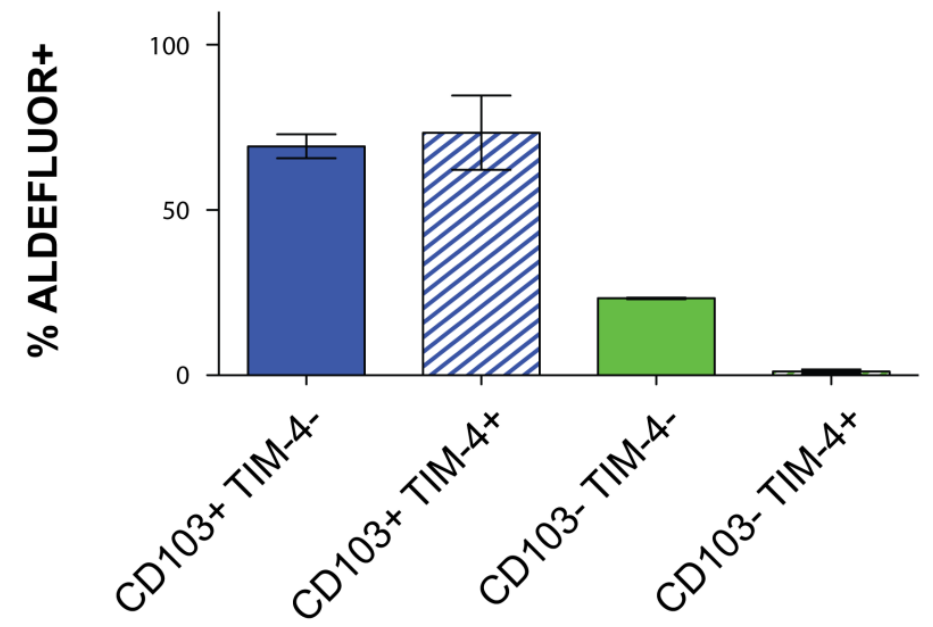

Figure 4.7: CD103+ TIM-4- and CD103+ TIM-4+ APC show high RALDH activity

LP APC were isolated 17 hours following intragastric OVA+CT. RALDH activity was determined using the ALDEFLUOR staining kit (A) and is shown for each subset (coloured line) compared to cells treated with RALDH inhibitor diethylaminobenzlaldehyde (DEAB) (shaded). (B) Frequency of RALDH positive cells within each APC subset. Bar graph shows combined mean \pm SEM from three independent experiments. 


\subsubsection{TLR4 signalling is not required for the increased frequency of CD103+ TIM- 4+ cells in the LP and MLN following oral administration of CT}

Following CT treatment, I found CD103+ TIM-4+ DC to increase in frequency and intestinal APC elevate expression of CD86 compared to steady-state. I was interested to investigate possible mechanisms by which this occurs. TLR signalling is important for DC sensing of commensal and pathogenic bacteria. TLR4 binds LPS, a component of bacterial cell walls and has also been suggested to be involved in DC activation in response to CT ${ }^{144}$. Furthermore, CT is known to cause epithelial barrier dysfunction, allowing for commensal bacteria to enter the LP and interact with DC. Therefore, I wanted to determine whether TLR4 was involved in the CT induced appearance of CD103+ TIM-4+ cells. To address this, OVA+CT was gavage fed to TLR4 deficient mice and CD103+ DC were analysed for TIM-4+ expression. I found the frequency of CD103+ TIM-4+ cells in the LP (Fig. 4.8, AB) and the MLN (Fig. 4.9, A-B) to be similar between TLR4-deficient and C57 mice following CT treatment suggesting that TLR4 signalling is not required for CT to induce the appearance of CD103+ TIM-4+ cells in the LP or MLN. Furthermore, expression of CD86 on APC subsets in the LP (Fig. 4.8, C-D) and MLN (Fig. 4.9, C-D) was not significantly different between TLR4 deficient and C57 mice treated with CT. Therefore, APC activation in response to CT was independent of TLR4 signalling. 


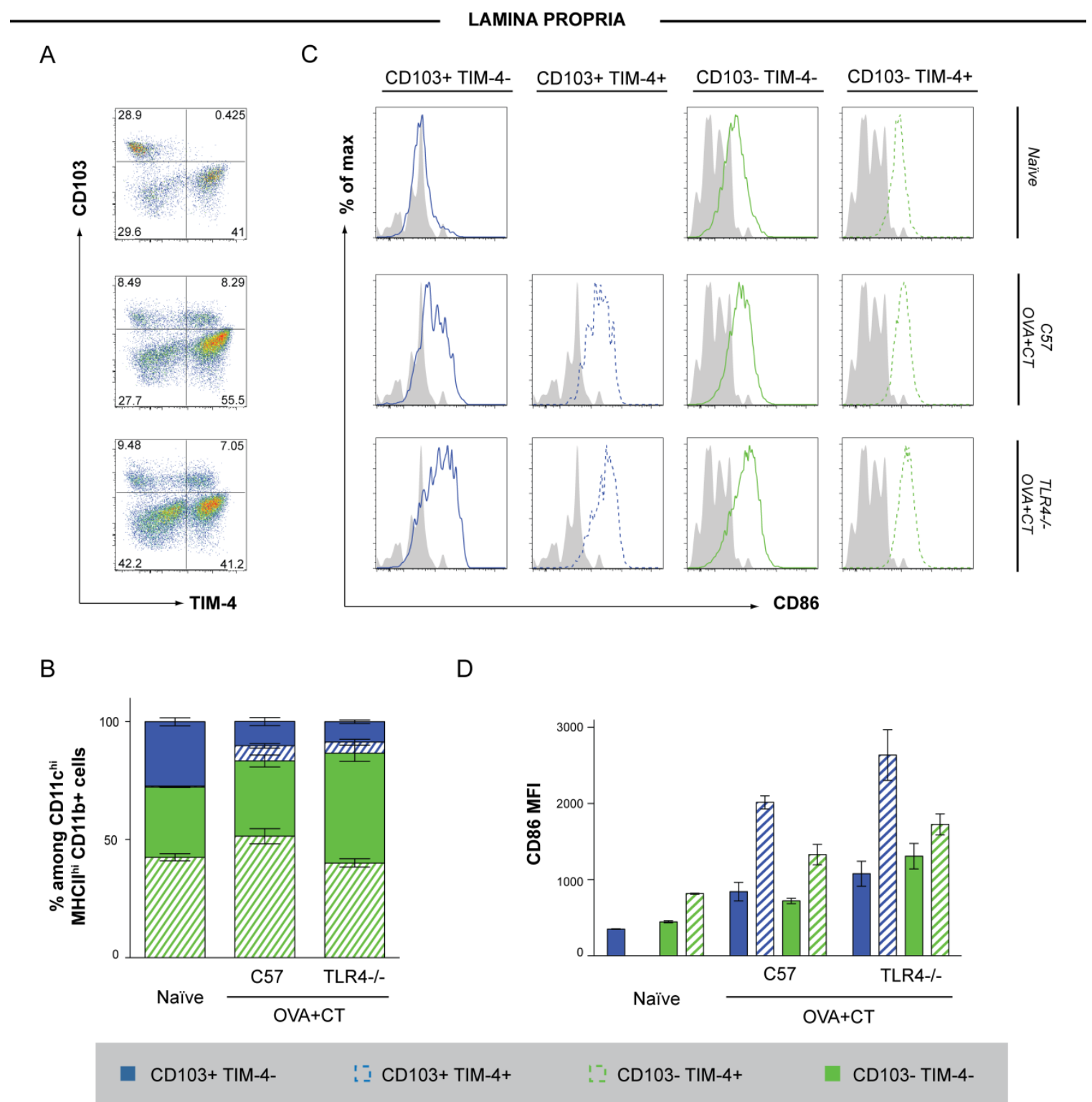

Figure 4.8: TLR4 is not required for the appearance of CD103+ TIM-4+ APC in the LP

LP cells were isolated 17 hours following intragastric OVA+CT from TLR4-/- mice. Frequency of APC subsets is shown on dot plots (A) and quantified on stacked bar graphs (B) compared to APC subsets from naïve TLR4-/- and OVA+CT treated C57 mice. To assess activation, CD86 expression is shown for each APC subset (coloured line) compared to FMO controls (shaded) on histograms (C) and quantified by CD86 MFI (D). Graphs show mean \pm SEM. 


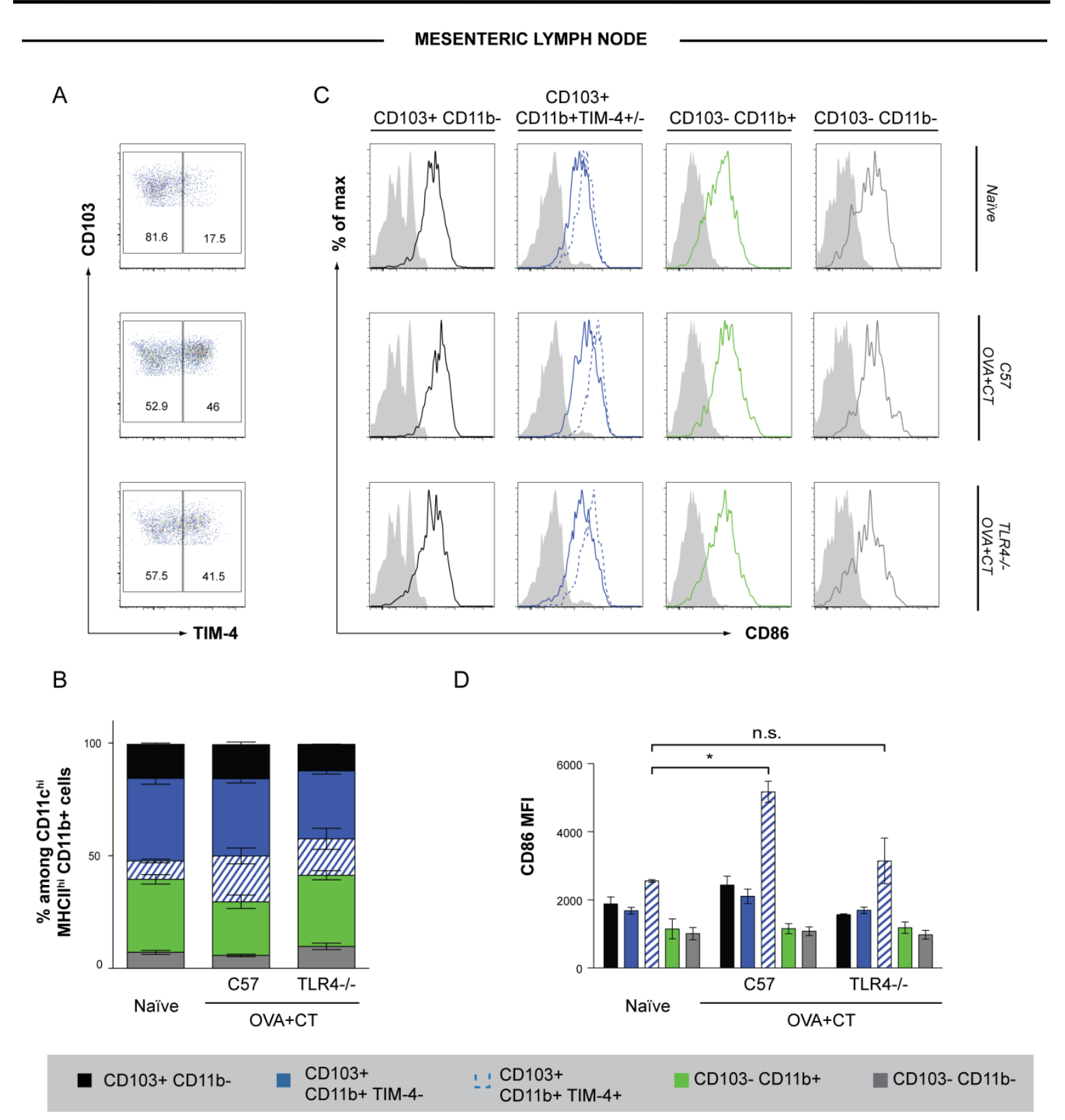

Figure 4.9: TLR4 is not required for the appearance of CD103+ TIM-4+ APC in the MLN

MLN cells were isolated 17 hours following intragastric OVA+CT from TLR4-/- mice. Frequency of APC subsets is shown on dot plots (A) and quantified on stacked bar graphs (B) compared to APC subsets from naïve TLR4-/- and OVA+CT treated C57 mice. To assess activation, CD86 expression is shown for each APC subset (coloured line) compared to FMO controls (shaded) on histograms (C) and quantified by CD86 MFI (D). Graphs show mean \pm SEM. 


\subsubsection{The increase in frequency of CD103+ TIM-4+ cells following oral CT is independent of intestinal commensal microbes}

Commensal bacteria in the intestine can influence DC phenotype and function via signalling through PRRs. Epithelial barrier dysfunction is associated with IBD, allowing for commensals to populate the LP. Therefore, movement of bacteria from the lumen to the LP due to CT induced barrier dysfunction may affect APC subset composition and activation. To determine whether commensal bacteria were involved in promoting the increase in frequency of CD103+ TIM-4+ cells, I supplemented drinking water with 5\% DSS for 3-5 days. In this model, by day 3, epithelial barrier integrity is compromised and commensals begin to populate the LP and by day 5 mice start to show symptoms of intestinal inflammation including the presence of fecal blood ${ }^{179}$. However, when I analysed small intestine LP APC subset compositions at 3 or 5 days following DSS treatment I did not detect any significant changes compared to naïve controls. Furthermore, CD103+ TIM-4+ cells could not be detected in the LP at the time points analysed (Fig. 4.10, A-B) and the expression of CD86 on APC subsets following DSS treatment was similar to the expression on APC subsets from control mice (Fig. 4.10, C-D). These results suggest that commensals alone are unable to promote the expression of TIM-4 and CD86 on CD103+ APC after DSS treatment.

To determine whether commensals play a role in altering DC subset composition when CT is administered, I treated mice with a pan antibiotic cocktail for 10 days, to significantly reduce microbes, prior to OVA+CT administration. Antibiotic treatment did not significantly alter steady-state APC composition or expression of CD86 (Fig. 4.11 and 4.12). Furthermore, there was no significant difference in the frequency of CD103+ TIM-4+ cells in the LP (Fig. 4.11, A-B) or MLN (Fig. 4.12, A-B) between mice treated with antibiotics and OVA + CT compared to control mice treated with OVA+CT. In addition, the expression of CD86 on intestinal APC from the LP (Fig. 4.11, C-D) and MLN (Fig. 4.12, C-D) did not differ significantly between OVA+CT treated mice with or without antibiotic treatment. These results suggest commensal bacteria are not playing a role in the appearance of CD103+ TIM-4+ cells or the activation of LP APC following CT treatment. 

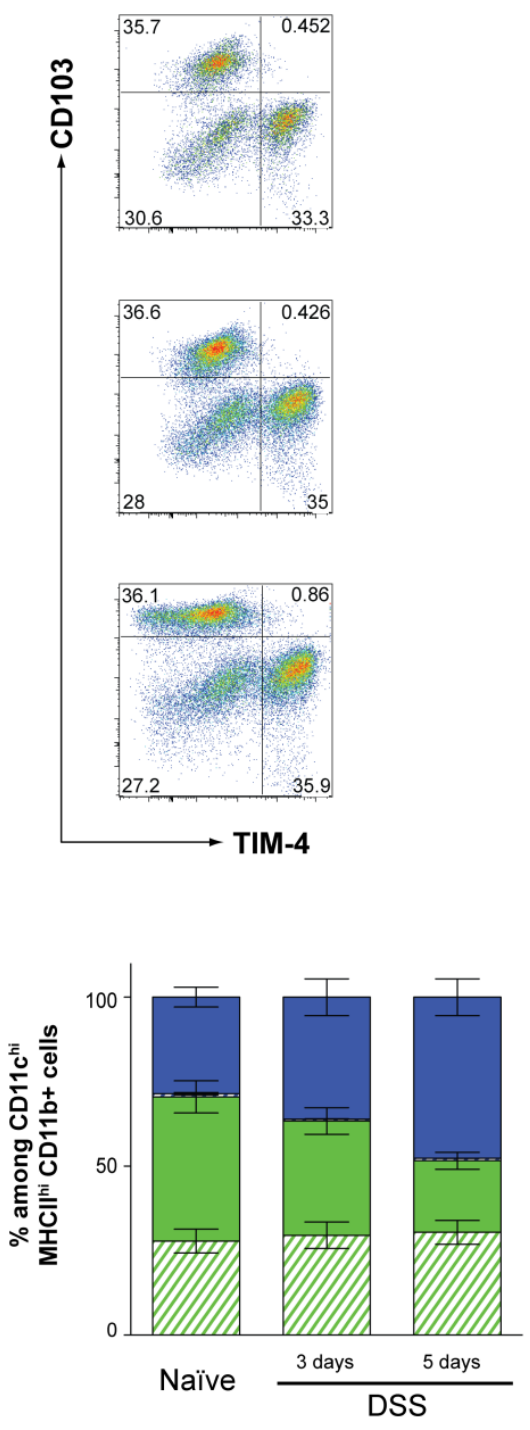
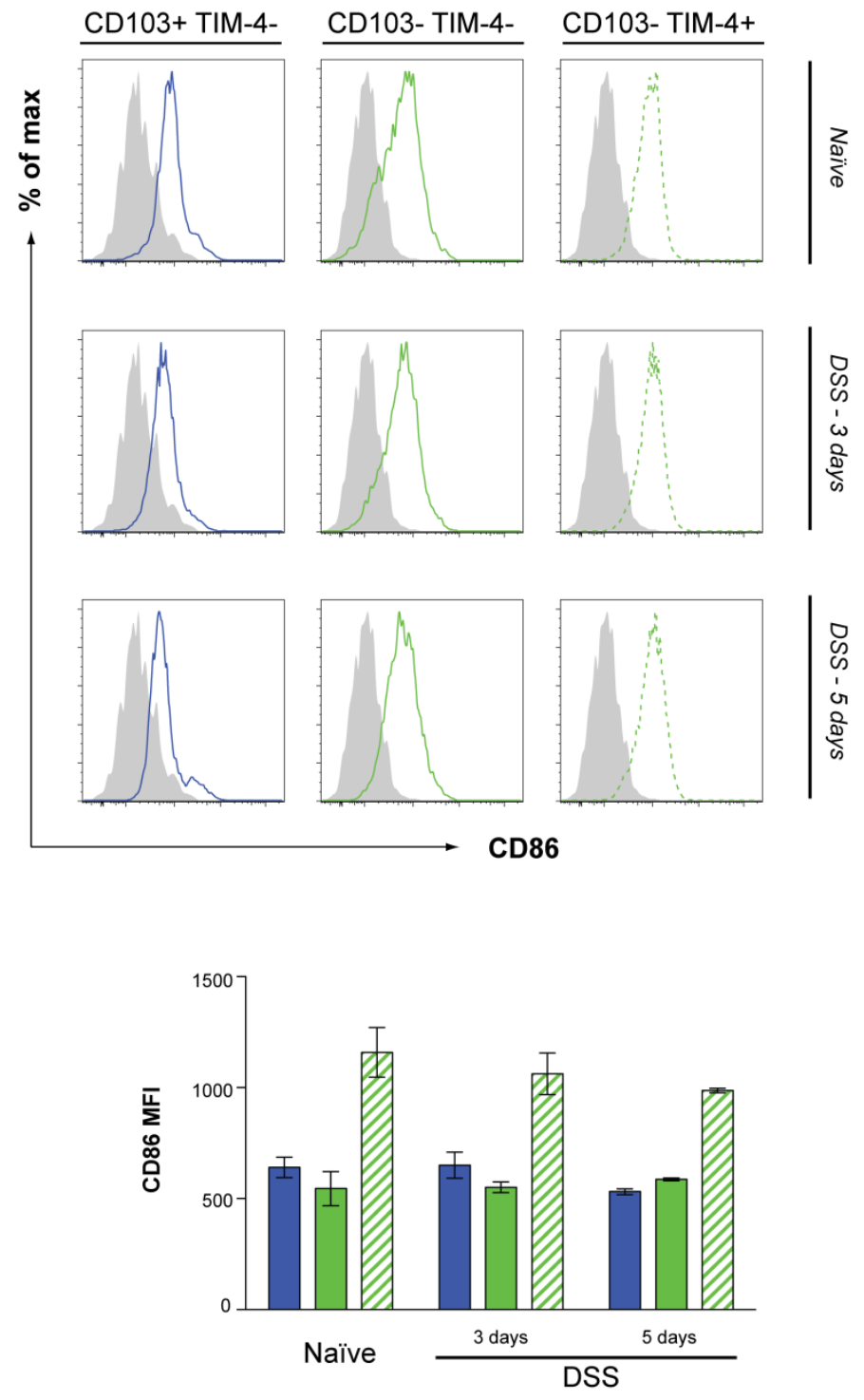

\section{CD103+ TIM-4- : : CD103+ TIM-4+ — CD103- TIM-4- : CD103- TIM-4+}

Figure 4.10: DSS induced colitis does not change small intestinal APC subset compositions significantly

LP APC were isolated from the small intestine of mice treated with DSS in their drinking water for 3 or 5 days. Frequencies of individual APC subsets are shown in dot plots (A) and quantified on a stacked bar graph (B). CD86 staining as shown for each APC subset (coloured line) compared to FMO controls (shaded) on histograms (C) was used to assess APC activation. Quantification was determined by CD86 MFI and shown on bar graphs. Graphs shown mean \pm SEM. Data is representative of three independent experiments. 


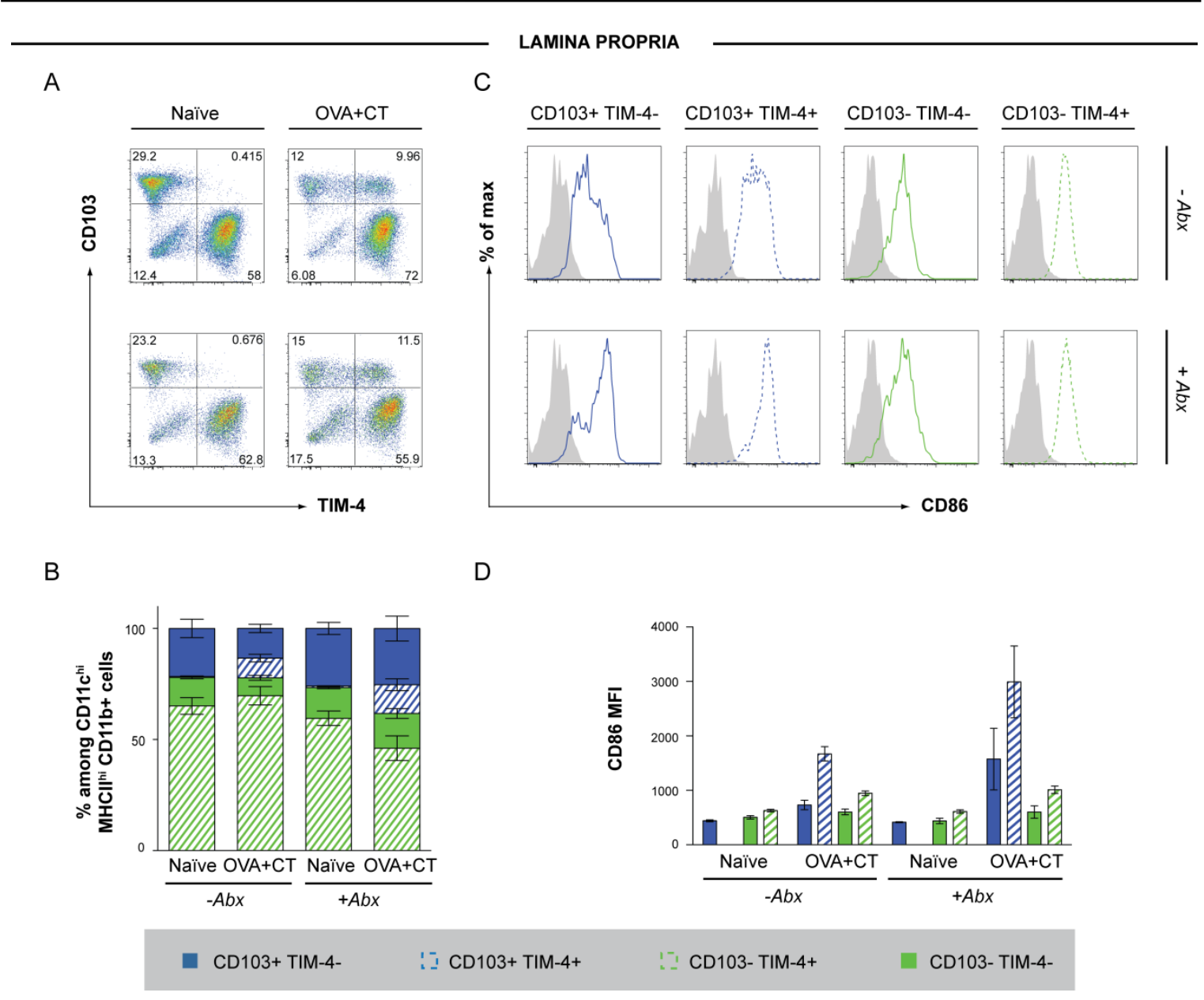

Figure 4.11: Commensal microbes are not required for the appearance of CD103+ TIM-4+ APC in the LP

Mice were treated with an antibiotic cocktail (ampicillin, gentamicin, neomycin, metronidazole and vancomycin) for 10 days prior to intragastric challenge with OVA+CT. LP cells were isolated 17 hours following intragastric OVA+CT. Frequency of APC subsets is shown on dot plots (A) and quantified on stacked bar graphs (B) compared to APC subsets from mice treated with or without antibiotics and OVA+CT. To assess activation, CD86 expression is shown for each APC subset (coloured line) compared to FMO controls (shaded) on histograms (C) and quantified by CD86 MFI (D). Graphs show mean \pm SEM. 
A

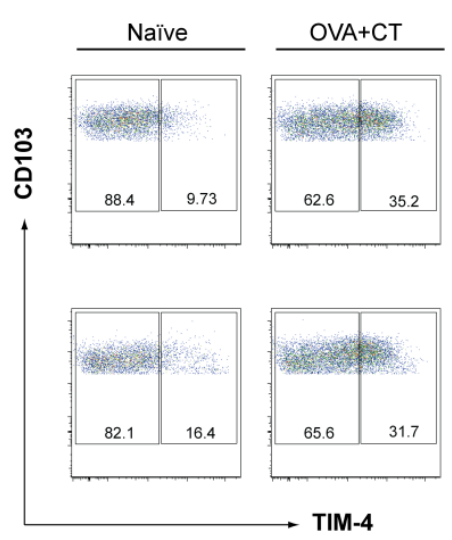

B

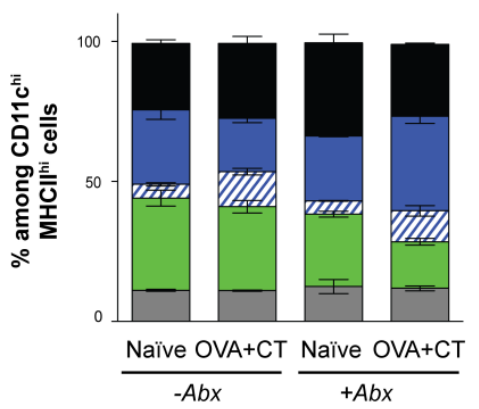

MESENTERIC LYMPH NODE

C

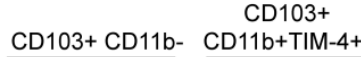
D11b+TIM-4+/-
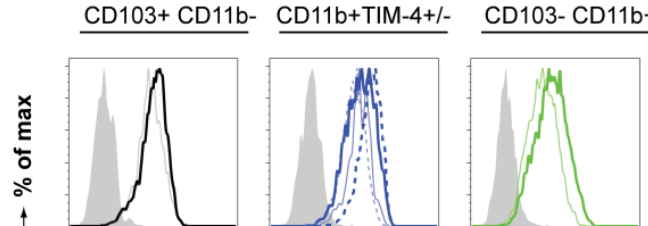

CD103- CD11b-
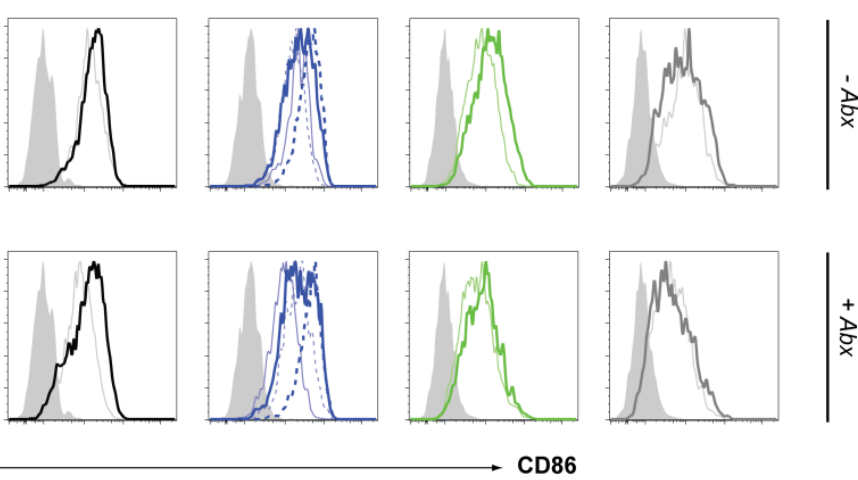

D

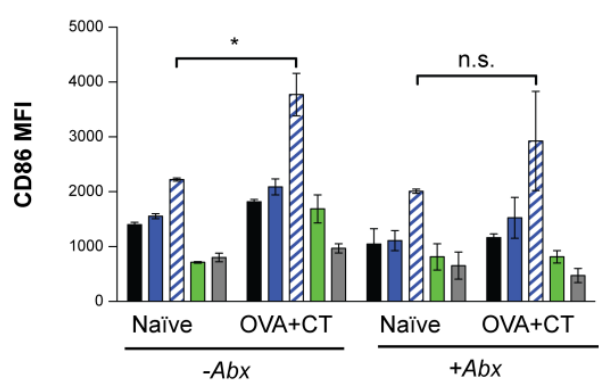

Figure 4.12: Commensal microbes are not required for the appearance of CD103+ TIM-4+ APC in the MLN

Mice were treated with an antibiotic cocktail (ampicillin, gentamicin, neomycin, metronidazole and vancomycin) for 10 days prior to intragastric challenge with OVA+CT. MLN cells were isolated 17 hours following intragastric OVA + CT. Frequency of APC subsets is shown on dot plots (A) and quantified on stacked bar graphs (B) compared to APC subsets from mice treated with or without antibiotics and OVA+CT. To assess activation, CD86 expression is shown for each APC subset following OVA+CT administration (bold coloured line) with CD103+ TIM-4+ cells indicated by a dashed line compared to APC subsets from naïve mice (coloured line) and FMO controls (shaded) on histograms (C) and quantified by CD86 MFI (D). Graphs show mean \pm SEM. 


\subsection{Discussion}

Intestinal APC subsets drive tolerogenic responses to food antigens and commensal bacteria while maintaining the ability to mount immune responses to pathogens. It is currently not well understood which subsets are responsible for the recognition and induction of adaptive immune responses in the intestine. Therefore, I wanted to assess the phenotype of intestinal DC following administration of a mucosal adjuvant to determine if there was any significant change in the compositions of APC subsets compared to steady-state. Following oral administration of CT with OVA antigen, I identified a novel phenotype of CD103+ DC which express the cell surface molecule TIM-4 and increased levels of the co-stimulatory molecule CD86. The presence of CD103+ TIM-4+ DC appeared to depend on the activity of CT but did not require signalling from commensal bacteria or signalling through TLR4. CD103+ TIM-4+ DC could also be detected in the MLN, suggesting these DC migrate from the LP and may influence $\mathrm{T}$ cell phenotype and intestinal immunity. Therefore, I propose that CD103+ TIM-4+ DC may contribute to the development of immune responses following oral administration of CT.

\subsubsection{CD103+ DC upregulate TIM-4 in response to CT}

Oral administration of the mucosal adjuvant CT results in the appearance of a novel phenotype of CD11b+ APC in the LP and MLN characterised by co-expression of CD103 and TIM-4. Currently, little is understood about the phenotype and function of intestinal APC subsets following administration of a mucosal adjuvant. At steady-state, there are three distinct populations of CD11b + APC in the LP which I classified as CD103+ TIM-4- DC, CD103- TIM-4+ M $\varphi$ and a heterogeneous population of CD103- TIM-4- cells which likely include DC and $\mathrm{M} \varphi$. I hypothesised that CD103+ TIM-4+ cells detected after CT treatment were arising from one of these resident APC populations as opposed to being recruited to the LP from the blood.

In support of my hypothesis I found that CD103+ TIM-4+ cells arise from resident CD103+ TIM-4- DC following oral administration of CT. Firstly, CT treatment did not alter 
the frequency of total CD103+ CD11b+ cells in the LP (Fig. 4.1). The frequency of CD103+ TIM-4+ cells increased as the frequency of CD103+ TIM-4- DC decreased suggesting that CD103+ DC upregulate TIM-4 in response to CT. Secondly, CD103+ TIM4- and CD103+ TIM-4+ DC had high activity of the RA metabolising enzyme RALDH compared to CD103- populations. RALDH activity is strongly associated with intestinal DC and thus detection of RALDH activity within the CD103+ TIM-4+ population suggests these cells possess similar specialised function as the intestinal CD103+ DC population (Fig. 4.6). Thirdly, CD103+ TIM-4+ cells do not express $M \varphi$ markers $\mathrm{CX}_{3} \mathrm{CR} 1, \mathrm{~F} 4 / 80$ or CD64. Therefore, it is unlikely CD103+ TIM-4+ cells are derived from the CD103- TIM-4+ resident $\mathrm{M} \varphi$ population or the CD103- TIM-4- population as they would have to lose expression of $\mathrm{CX}_{3} \mathrm{CR} 1, \mathrm{~F} 4 / 80$ and CD64 as well as gain activity of RALDH and expression of CD103. Finally, as CD103+ TIM-4+ cells do not express CD64, it is unlikely they originate from monocytic precursors from the blood as inflammatory DC have been shown to express CD64 ${ }^{50}$. Together these data suggests CD103+ DC upregulate TIM-4 in response to oral CT.

Expression of TIM-4 has been reported on $\mathrm{M} \varphi$ populations in the lung ${ }^{145}$ and peritoneum ${ }^{146}$ but has not been previously reported on DC populations in vivo. However, in vitro studies have shown CT and other DAMPs such as LPS can induce DC maturation and upregulation of TIM-4 ${ }^{147,}{ }^{148}$. Thus, expression of TIM-4 may indicate DC maturation in vivo and may affect DC functions such as $\mathrm{T}$ cell priming capabilities. Further evidence to support this conclusion is the fact that CD103+ TIM-4+ cells express higher levels of the activation marker CD86 compared to other APC populations in the LP and total CD103+ populations from naïve mice. Although it is not clear from these studies, it would be of interest to determine whether a distinct subset of CD103+ DC upregulate TIM-4 in response to CT or whether CD103+ DC that have directly or indirectly interacted with CT upregulate TIM-4. 


\subsubsection{CT induced TIM-4 upregulation by CD103+ DC does not require signalling through TLR4 or from commensal bacteria}

Recognition of MAMPs and DAMPs via PRRs such as TLRs can influence APC phenotype and function ${ }^{144,}{ }^{148}$. In the intestine, signalling through PRRs as well as signals from commensal bacteria are important to maintain immune homeostasis at steady-state ${ }^{64,65,66}$. Germ-free mice are deficient in these signals, and have high serum $\operatorname{IgE}$ titres and are prone to developing allergic diseases compared to littermate controls ${ }^{94}$. In contrast, prolonged PRR signalling from commensals and direct interaction of commensals with APC has been implicated in the pathogenesis of IBD ${ }^{178,180}$. Thus, a unique paradigm exists in the intestine where PRR signals are required for the development of tolerance as well as inflammatory responses.

In vitro studies have reported DC to mature and upregulate TIM-4 in response to DAMPs such as LPS. Others have suggested a key role for TLR4 signalling in CT induced TIM-4 upregulation ${ }^{144}$. In addition, $\mathrm{CT}$ is known to compromise epithelial barrier function allowing for movement of commensals into the LP and interact with APC ${ }^{177}$. Therefore, I hypothesised that CD103+ DC may be upregulating TIM-4 expression in response to commensal microbial components through PRRs such as TLR4 following CT treatment. In contrast to my hypothesis, TIM-4 upregulation by CD103+ DC was independent of both TLR4 signalling and commensal bacteria as shown by experiments using TLR4 receptor deficient mice and antibiotic treatment. Although oral feeding of an antibiotic cocktail is not likely to deplete all bacteria from the intestine, treated mice had similar characteristics to germ-free mice including enlarged ceca, which would suggest the depletion was effective. Furthermore, introduction of commensals into the LP following DSS treatment failed to induce TIM-4 upregulation by DC. It is important to note the possibility that very few bacteria may translocate into the small intestinal LP. Although bacterial translocation was not measured in this study, reports have shown that the upper small intestine has a lower bacterial load compared to other regions of the intestine ${ }^{181}$. Together, these experiments 
would suggest that upregulation of TIM-4 on CD103+ DC depends on the activity of CT, rather than indirect action by microbiota.

This is the first report of TIM-4 expression on a DC population in vivo. Therefore, it is possible that intestinal resident DC require different signals to upregulate TIM-4 in response to CT compared to in vitro generated BMDC that were studied in published reports ${ }^{144}$. A recent study has shown that ganglioside signalling by epithelial cells and DC is the predominant mechanism by which intestinal DC mature in response to CT in vivo ${ }^{160}$. Thus, ganglioside signalling may be involved in prompting TIM-4 upregulation by CD103+ DC in addition to maturation signals.

\subsubsection{CD103+ TIM-4+ DC are present in the lymph node}

CD103+ TIM-4+ DC were present in the lymph node from 12 hours post OVA+CT treatment. This suggests that CD103+ TIM-4+ DC will have the opportunity to interact with $\mathrm{T}$ cells and thus, may influence immune responses. CD103+ TIM-4- DC have been shown to have the capacity to migrate to the MLN ${ }^{42}$ which is critical for the induction of immune responses to oral antigen ${ }^{101}$. Furthermore, mice deficient in the lymph node homing receptor CCR7 have been shown to have a decreased frequency of CD103+ $\mathrm{CD} 11 \mathrm{~b}+$ cells in the MLN ${ }^{111}$ and fail to mount immune responses following oral immunisation ${ }^{168}$.

The data presented in this Chapter suggests that CD103+ TIM-4+ DC are derived from the migratory CD103+ TIM-4- population. If this is the case, CD103+ TIM-4- and CD103+ TIM-4+ DC may share similar migratory capabilities. Time course experiments revealed that CD103+ TIM-4+ DC appear in the LP and MLN from eight and twelve hours post OVA+CT respectively. This data suggests that following OVA+CT treatment the LPresident CD103+ upregulate TIM-4 and subsequently migrate to the MLN where they can be detected 4 hours later. Thus, CD103+ TIM-4+ DC may play a role in the driving effector immune responses to oral antigen in the presence of CT. 


\subsection{Conclusions}

The composition of intestinal APC following administration of a mucosal adjuvant and the role of individual APC subsets in driving effector T cell responses are not well understood. In this chapter, I demonstrate a proportion of CD103+ DC alter their phenotype and morphology in response to CT induced inflammation. This population can be characterised by co-expression of CD103 and TIM-4 and elevated expression of the activation marker CD86. These changes in phenotype appear to be independent of TLR4 signalling and DC interaction with commensal bacteria, but require CT. In addition, CD103+ TIM-4+ DC appear to have the capacity to migrate to the MLN and thus may influence T cell responses. I propose CD103+ TIM-4+ DC may be playing a role in influencing intestinal immune responses. Further study of this subset may provide insight into processes contributing to intestinal pathologies such as food allergy and IBD. 


\section{Chapter 5: Orally administered cholera toxin leads to functional changes among intestinal immune cells}




\subsection{Introduction}

CT can promote the induction of CD4 effector immune responses to co-delivered antigen. Interestingly, there is significant experimental evidence showing that CT promotes a biased Th2 response 155, 156 150, 154 . Models using CT as an adjuvant have been associated with induction antigen specific IL-4 producing T cells 155, 156, 162 and inhibition of Th1 proinflammatory cytokine production by DC ${ }^{150,182,183}$. For this reason, CT has been used as an adjuvant to sensitise mice to allergens in models of food allergy ${ }^{184,185}$ which are mediated by Th2 immunity. However, others claim that CT provides general stimulatory signals that result in activation of $\mathrm{NF} \varkappa \mathrm{B}$ in local immune cells leading to production of proinflammatory cytokines ${ }^{157}$. Furthermore, DC vaccine studies suggest that CT can promote the development of Th1 and Th2 responses simultaneously as DC pulsed with CT and antigen induced IFN $\gamma$ production by antigen specific $\mathrm{T}$ cells in vitro ${ }^{151,155}$. Therefore, I was interested to assess cytokine production by antigen specific $\mathrm{T}$ cells following oral coadministration of CT and OVA antigen.

In addition to influencing $\mathrm{T}$ cell responses, CT has been shown to affect the phenotype and functions of intestinal APC 144, 150,153, 154, 158, 159, 161, 186. In Chapter 4 I showed a proportion of intestinal CD103+ DC upregulated the cell surface protein TIM-4 and the activation marker CD86 following CT treatment. In light of these observed phenotypic changes, I was interested to investigate whether CT treatment also altered the functions of intestinal APC. Intestinal CD103+ DC have the specialised capacity to metabolise RA which promotes Treg development at steady-state ${ }^{57,58}$. However, in a perturbed state such as in a model of colitis, expression of RA metabolising enzyme RALDH is significantly decreased ${ }^{175}$. Therefore, I was interested to determine what impact oral administration of CT would have on RALDH activity among CD103+DC from the intestine and MLN.

In order for intestinal APC to carry out their function as antigen presenting cells they need to be able to acquire antigen. Interestingly, previous studies have failed to show antigen uptake by CD103+ DC after oral delivery under homeostatic conditions ${ }^{42,56}$. However, 
following infection with Salmonella, CD103+ DC were shown to migrate to the epithelial barrier and take up bacteria ${ }^{70,72}$. Furthermore, administration of CT has been shown to promote recruitment of CD103+ DC to the epithelial barrier ${ }^{158}$, a location more proximal to luminal antigen. Thus, I hypothesised that CD103+ DC, in particular DC that had responded to CT by upregulating TIM-4, may have an increased capacity to take up luminal antigen following oral administration of CT.

As I was interested to explore functional changes in the APC populations after CT treatment, I investigated the role of TIM-4 expression on CD103+ DC. TIM-4 expression on $\mathrm{M} \varphi$ has been shown to bind a number of co-ligands including phosphatidylserine (PtdSer) expressed on the surface of apoptotic cells which promotes their uptake ${ }^{187,188}$. However, it is not known whether DC expressing TIM-4 obtain the same ability to take up apoptotic material. Therefore, I was interested to compare the apoptotic cell uptake capacity of CD103+ DC that express TIM-4 following CT treatment to CD103+ TIM-4- DC.

It is clear that oral administration of CT influences the phenotype and functions of intestinal immune cells. Thus, in this Chapter, I wanted to investigate the effect of orally administered CT on $\mathrm{T}$ cell immune responses in the intestine and, specifically, the function of CD103+ TIM-4+ DC. From these studies, I found CT treatment promoted antigen specific T cell expansion and production of IL-4. Furthermore, while there was no difference in RALDH activity among CD103+ DC isolated from CT treated and untreated mice, the capacity to take up apoptotic material ex vivo and antigen in vivo was enhanced among CD103+ DC that expressed TIM-4 following CT treatment. Together this data demonstrates that oral administration of CT induces functional changes among intestinal APC and induces IL-4 production by CD4+ T cells. 


\subsection{Aims}

Immunisation using CT as an adjuvant had been associated with driving effector $\mathrm{T}$ cell responses to co-delivered antigen. I have shown CT affects the phenotype of intestinal APC however, it is unclear whether this change in phenotype is associated with functional changes. Therefore, I was interested to assess how oral administration of CT affects the function of intestinal T cells and APC.

Specifically my aims were:

1. To determine how oral administration of $\mathrm{CT}$ with antigen affects antigen specific $\mathrm{T}$ cell expansion and cytokine production in the LP, MLN and spleen.

2. To determine whether CT administration is associated with a change in RALDH activity among intestinal APC subsets.

3. To determine if CD103+ DC that have upregulated TIM-4 in response to CT have an increased capacity to take up apoptotic cells and antigen. 


\subsection{Results}

\subsubsection{OVA $+\mathrm{CT}$ induces antigen specific $\mathrm{T}$ cell proliferation and activation}

As CT is a known mucosal adjuvant, I was interested to assess CD4 T cell responses following oral administration of OVA antigen with CT. To assess this, mice were injected with CD4+ 4C13R OTII cells which express a TCR that specifically recognises the OVA peptide $\mathrm{OVA}_{323-339}$ and the fluorescent reporters, AmCyan under the IL-4 promoter and DS-

Red under the IL-13 promoter. One day following T cell transfer, mice were treated with CT or OVA+CT by oral gavage. Analysis of T cell proliferation and activation was carried out by flow cytometry seven days after transfer as shown in Fig. 5.1. The results show that the presence of antigen induced a modest increase in the frequency of transferred cells (as determined by CD45.2 staining) among total live cells in the LP and MLN but not the spleen (Fig. 5.2, A, C). Furthermore, expression of T cell activation marker CD44 was upregulated on transferred cells from OVA+CT treated mice from the LP, MLN and spleen compared to transferred cells from CT treated mice (Fig. 5.2, B, D). This data shows that coadministration of CT and antigen via the oral route induces antigen specific $\mathrm{T}$ cell proliferation and activation particularly in the LP and MLN.

\subsubsection{CT increases the frequency of IL-4 producing $T$ cells in the LP, MLN and spleen}

Immunisation with the adjuvant $\mathrm{CT}$ has been reported to preferentially induce differentiation of naïve CD4+ $\mathrm{T}$ cells to a Th2 phenotype ${ }^{150,155,156}$. Th2 cells are characterised by production of the cytokines IL-4, IL-5 and IL-13. Therefore, I wanted to investigate whether oral administration of CT with OVA antigen induced an antigen specific Th2 immune response. To address this, I measured the expression of the reporter AmCyan expressed by OTII cells 6 days after OVA+CT treatment (Fig. 5.1). The results show that IL-4 producing OTII cells could be detected in the LP (Fig. 5.3, C), MLN (Fig. 5.3, A) and spleen (Fig. 5.3, B) from mice treated with OVA+CT. However, little to no IL-4 producing 
cells could be detected among transferred cells from mice treated with CT alone. No IL-13 expression by the reporter DS-Red could be detected in this experiment (data not shown). These results suggest that CT increases the percentage of antigen specific IL-4 producing T cells in the LP, MLN and the spleen.

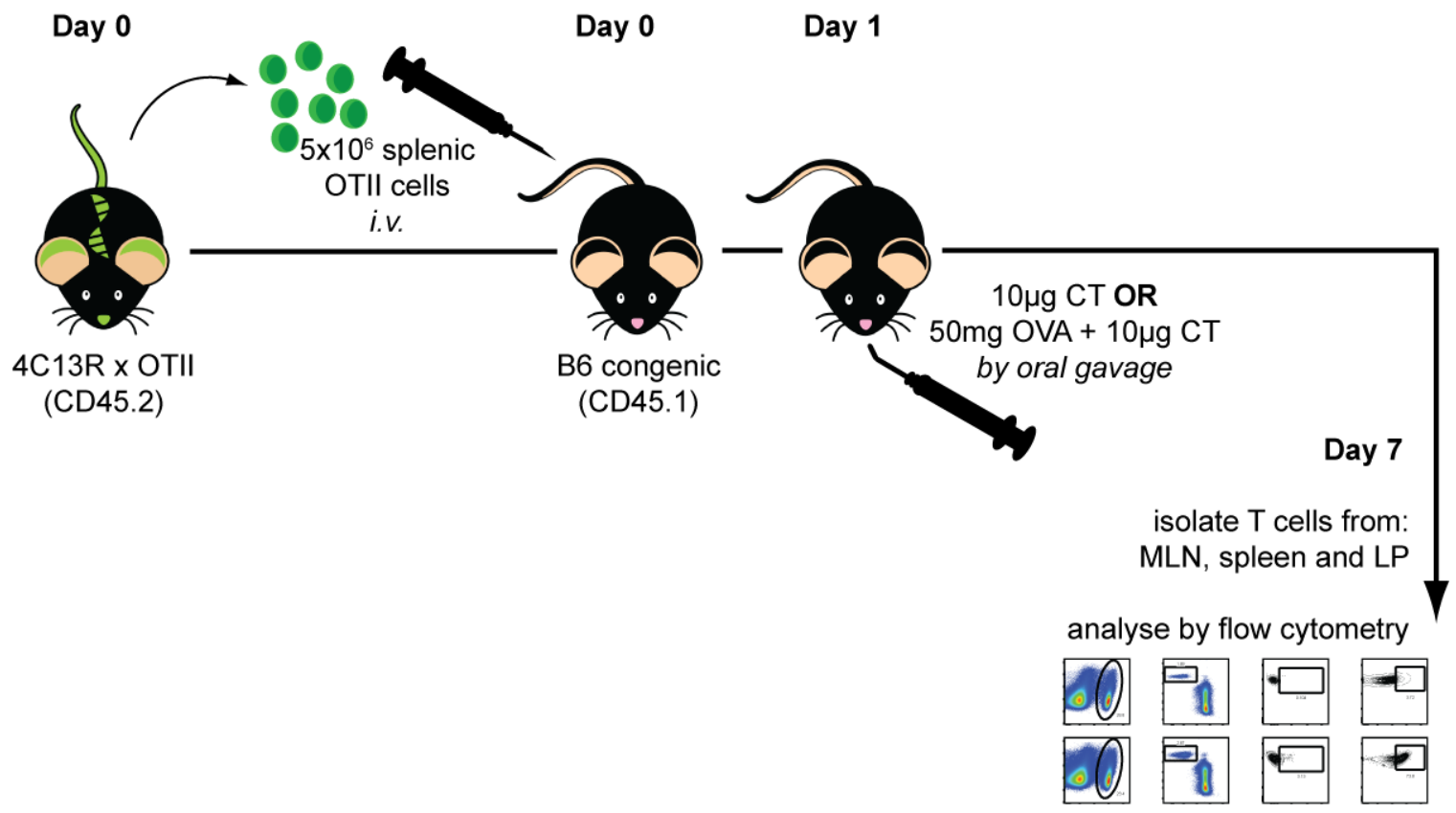

Figure 5.1: Experimental protocol used to analyse antigen specific $T$ cell responses following oral administration of OVA $+\mathrm{CT}$. 

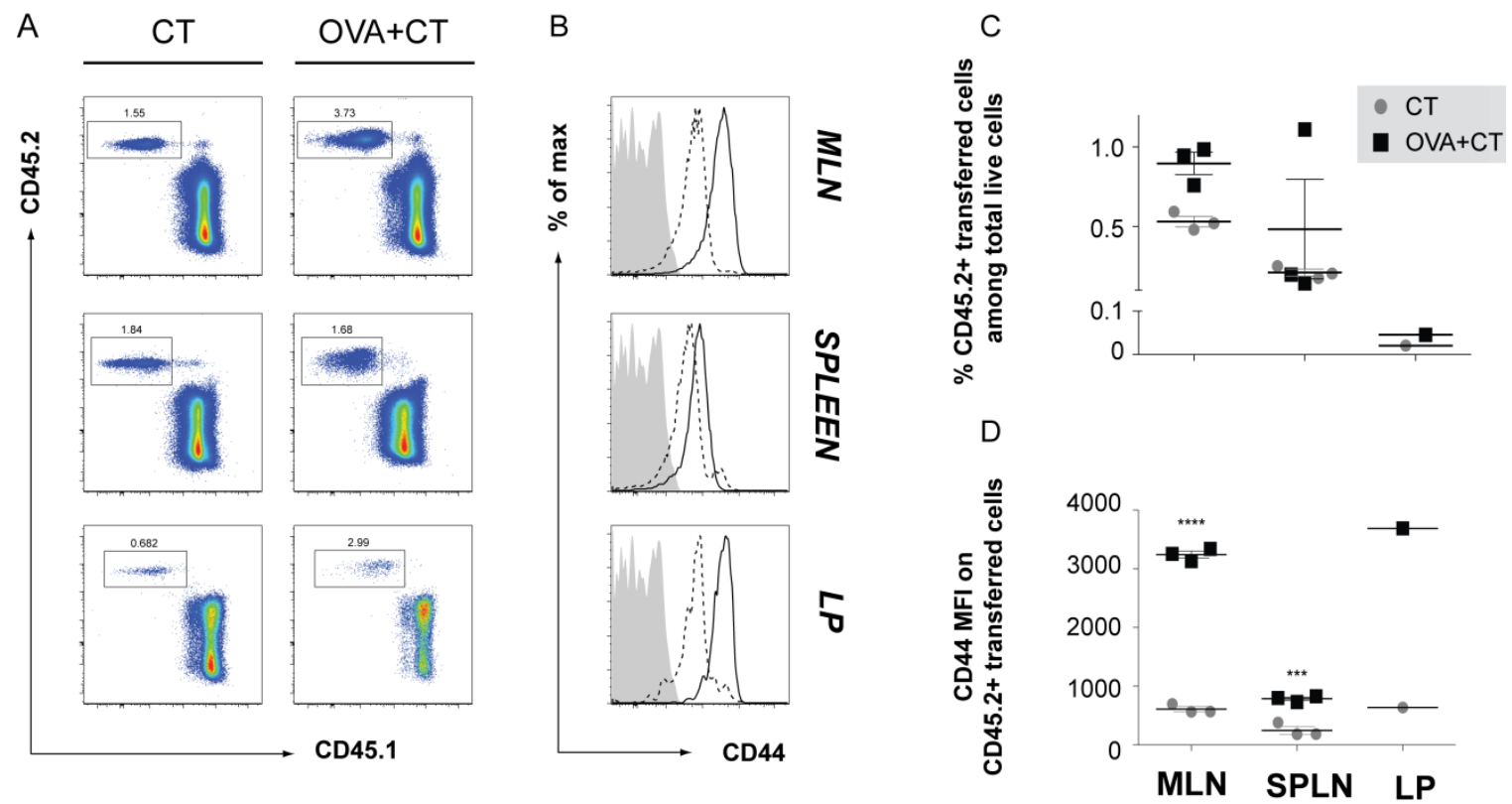

Figure 5.2: $\mathrm{CT}$ increases the frequency and activation of antigen specific $\mathrm{T}$ cells in the LP and MLN.

B6 congenic mice were injected i.v. with $5 \times 10^{6}$ CD45.2+ 4C13R OTII splenocytes enriched by magnetic selection. One day later mice were treated with $10 \mu \mathrm{g}$ CT or $50 \mathrm{mg}$ OVA $+10 \mu \mathrm{g}$ CT by oral gavage. On day 7, T cells were isolated from the LP, MLN and spleen. Frequency of transferred OTII cells among total CD4+ cells is shown on dot plots (A) and graph (C). Expression of CD44 is shown on histograms for OVA+CT (solid line) and CT treated mice (dashed line) (B). Graph shows CD44 MFI on transferred cells from each tissue (D). Graphs show mean \pm SEM. Statistical significance was determined by Two-Way ANOVA with Bonferroni post-test. ${ }^{* * *} 0.0001<\mathrm{p}<0.001$, $* * * * \mathrm{p}<0.0001$ 


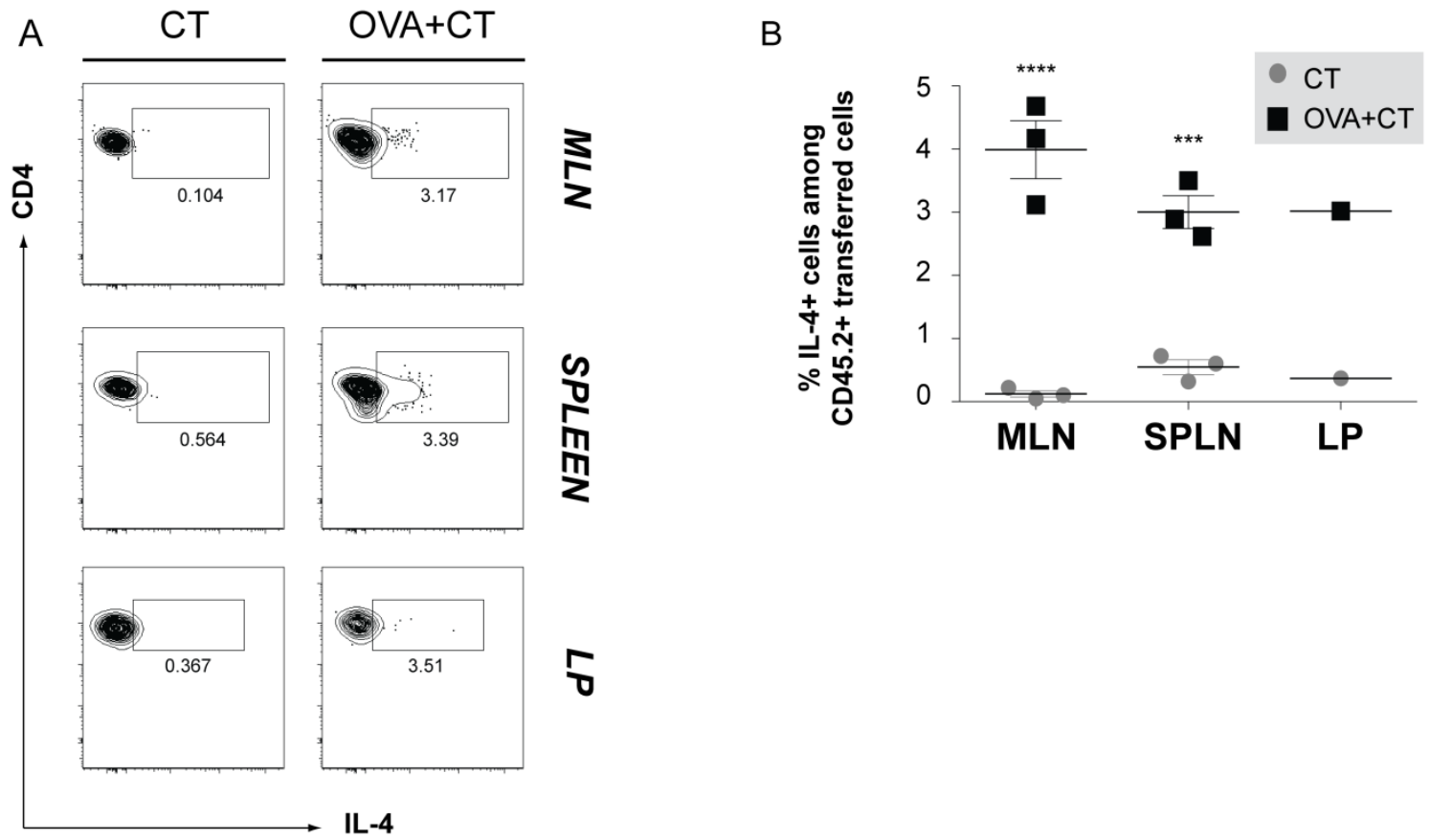

Figure 5.3: CT increases the frequency of IL-4 producing antigen specific $\mathrm{T}$ cells.

B6 congenic mice were injected i.v. with $5 \times 10^{6}$ CD45.2+ 4C13R OTII splenocytes enriched by magnetic selection. One day later mice were treated with $10 \mu \mathrm{g}$ CT or $50 \mathrm{mg}$ OVA+10 $\mu \mathrm{g}$ CT by oral gavage. On day 7, T cells were isolated from the LP, MLN and spleen and assessed for IL-4 production by the AmCyan reporter. Representative contour plots show production of IL-4 by OTII transferred cells (A). Graph shows percent IL-4+ cells among transferred cells (B) Graphs show mean \pm SEM. Statistical significance was determined by Two-Way ANOVA with Bonferroni posttest. ${ }^{* * *} 0.0001<\mathrm{p}<0.001, * * * * \mathrm{p}<0.0001$ 


\subsubsection{CT enhances antigen specific $\mathbf{T}$ cell proliferation but not activation}

While co-administration of OVA $+\mathrm{CT}$ was able to induce $\mathrm{T}$ cell proliferation and activation compared to administration of CT alone, I wanted to determine whether CT enhanced this process compared to administration of antigen alone. Therefore, mice were injected with CD4 OTII cells one day before oral administration of CT and OVA in combination or alone. On day 6, mice were challenged with OVA antigen by intragastric or intrapertioneal routes to boost immune responses (Fig. 5.4). On day 7, MLN and spleen OTII cells were analysed by flow cytometry for expansion and activation. The results show that OVA treated mice had 0.8-1.8\% OTII cells among total CD4+ cells in the MLN compared to 0.8-0.9\% from mice treated with CT. When OVA and CT were co-administered the frequency of OTII cells in the MLN increased to approximately $2.5 \%$ in mice challenged intragastrically and $1.5 \%$ in mice challenged intraperitoneally (Fig. 5.5, A-B). In the spleen, there was no significant difference in the frequency of OTII cells between treatment groups. All animals treated with OVA antigen showed elevated levels of T cell activation marker CD44 on OTII cells isolated from the MLN compared to animals treated with CT alone. Furthermore, although the frequency OTII cells in the spleen did not increase after antigen administration, CD44 expression was upregulated on splenic OTII cells from OVA and OVA+CT treated mice compared to CT treated group (Fig. 5.5, C-D). Together this data shows administration of OVA in the presence or absence of CT led to upregulation of CD44 on OTII cells, which is a marker of antigen experience, and that the addition of CT promoted a greater expansion of antigen specific $\mathrm{T}$ cells following antigen administration. 

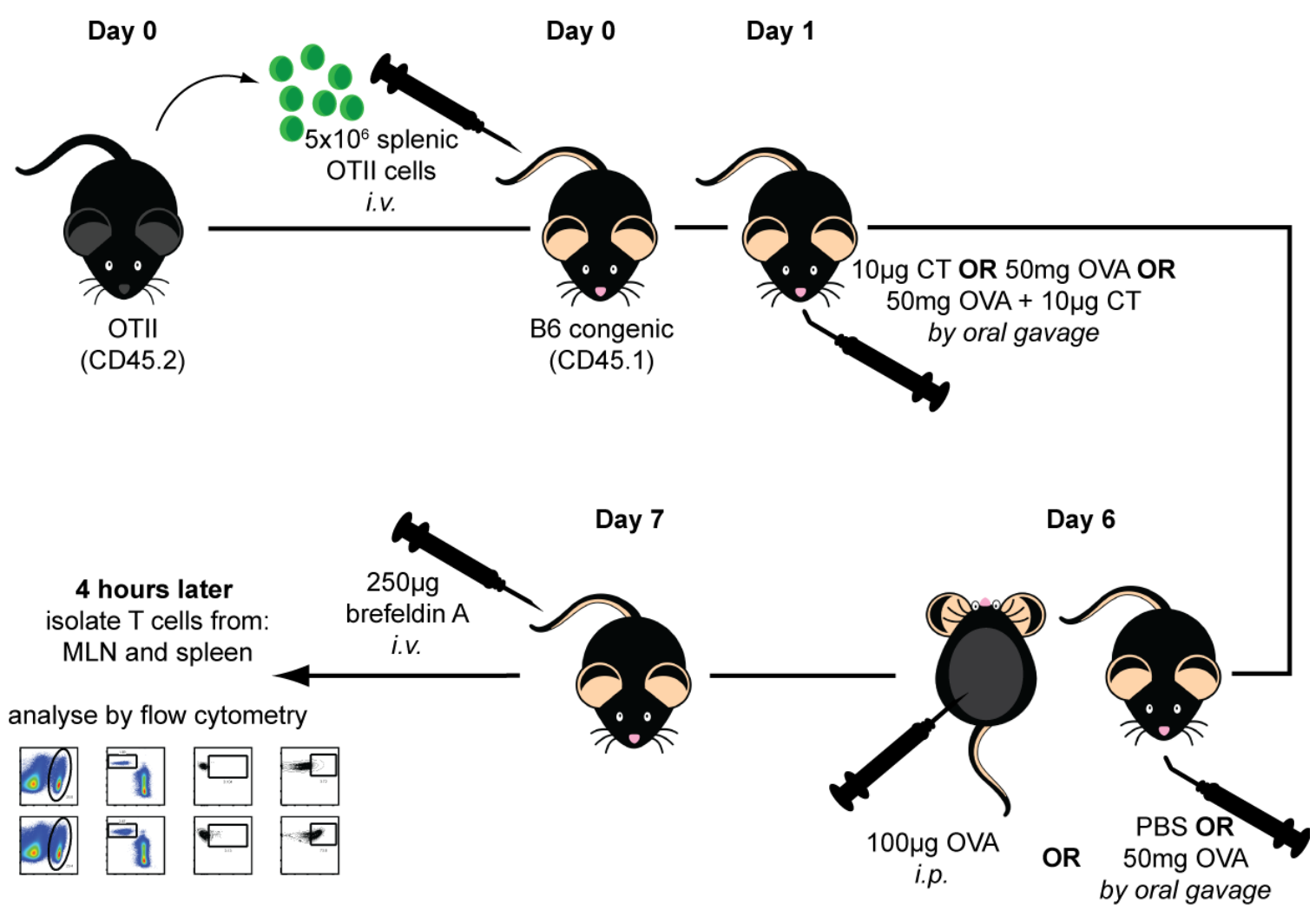

Figure 5.4: Experimental protocol used to analyse antigen specific $T$ cell responses by intracellular cytokine staining following oral administration of OVA+CT. 

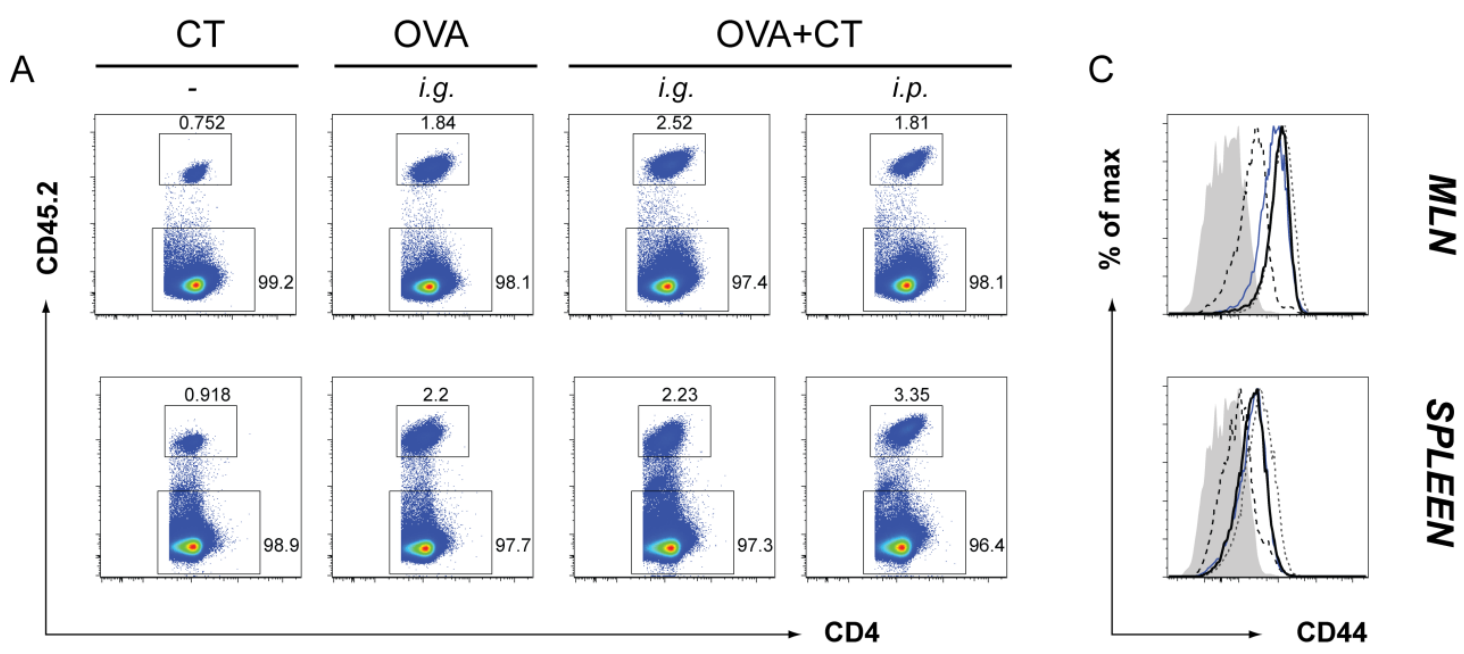

B

$M L N$

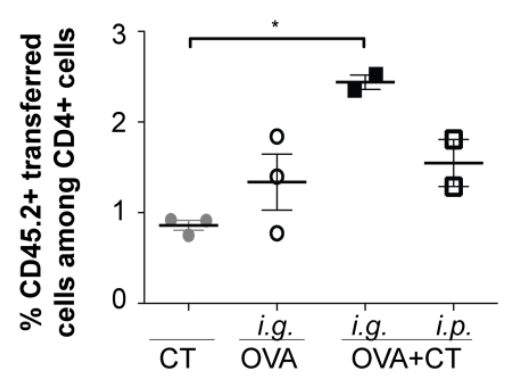

SPLEEN

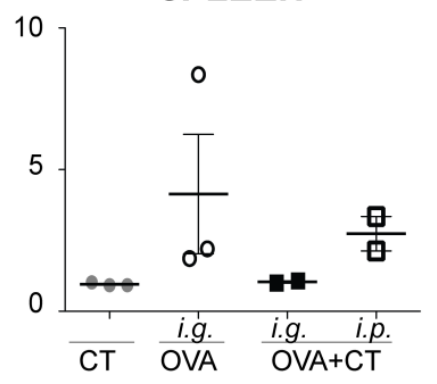

$\mathrm{D}$

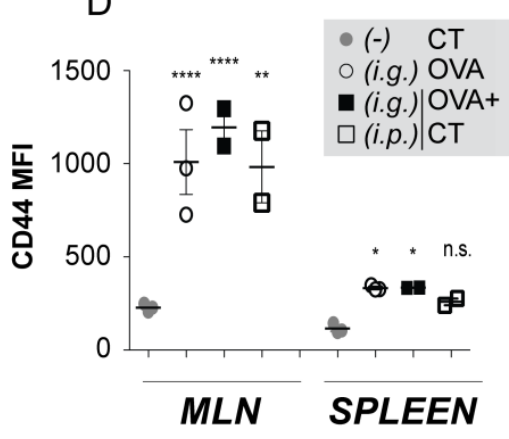

Figure 5.5: CT enhances antigen specific $T$ cell expansion in the MLN and spleen.

B6 congenic mice were injected i.v. with $5 \times 10^{6}$ CD $45.2+$ OTII splenocytes enriched by magnetic selection. One day later mice were treated with $10 \mu \mathrm{g}$ CT or $50 \mathrm{mg}$ OVA or a combination by oral gavage. On day 6, mice primed with OVA were given a second dose of OVA by oral gavage $(50 \mathrm{mg})$ or by intraperitoneal injection $(100 \mu \mathrm{g})$. On day 7 , the MLN and spleen were analysed for frequency of transferred cells and expression of CD44. Representative dot plots (A) and graphs (B) show the frequency of transferred cells among total CD4+ cells. Expression of CD44 on transferred cells from OVA+CT treated mice challenged i.g. (black solid line), or i.p. (blue solid line), and from OVA (dashed grey line) or CT alone (dashed black line) is shown on histograms (C). MFI of CD44 on transferred cells is shown on graph (D). Graphs show mean \pm SEM. Statistical significance was determined by Two-Way ANOVA with Bonferroni post-test. ${ }^{*} \mathrm{p}<0.05 * * \quad 0.001<\mathrm{p}<0.05 * * * *$ $\mathrm{p}<0.0001$ 


\subsubsection{T cell derived IFN $\gamma$ and IL-4 could not be detected by ICS following CT treatment}

In addition to driving Th2 immune responses, reports have shown CT used in a DC vaccine context drive both Th1 and Th2 responses ${ }^{151}$. This data suggests CT may provide general signals to enhance the capacity of DC to drive immune responses. In my study, I observed the appearance of an activated population of DC following oral CT, therefore, it was of interest to assess IFN $\gamma$ production by antigen specific $T$ cells in mice treated with OVA+CT. To assess cytokine responses, on day 7 mice were injected with Golgi stop agent brefeldin A, which blocks release of cytokine from the cell in vivo. Four hours after treatment mice were sacrificed and cells from the MLN and spleen were isolated and cytokine production was assessed by intracellular cytokine staining (ICS) (Fig. 5.4). The results showed little to no production of IFN $\gamma$ (Fig. 5.6, C-D) or IL-4 (Fig. 5.6, A-B) above background by splenic or MLN OTII cells. Therefore, I could not draw any conclusions on the cytokine response from this experiment. 


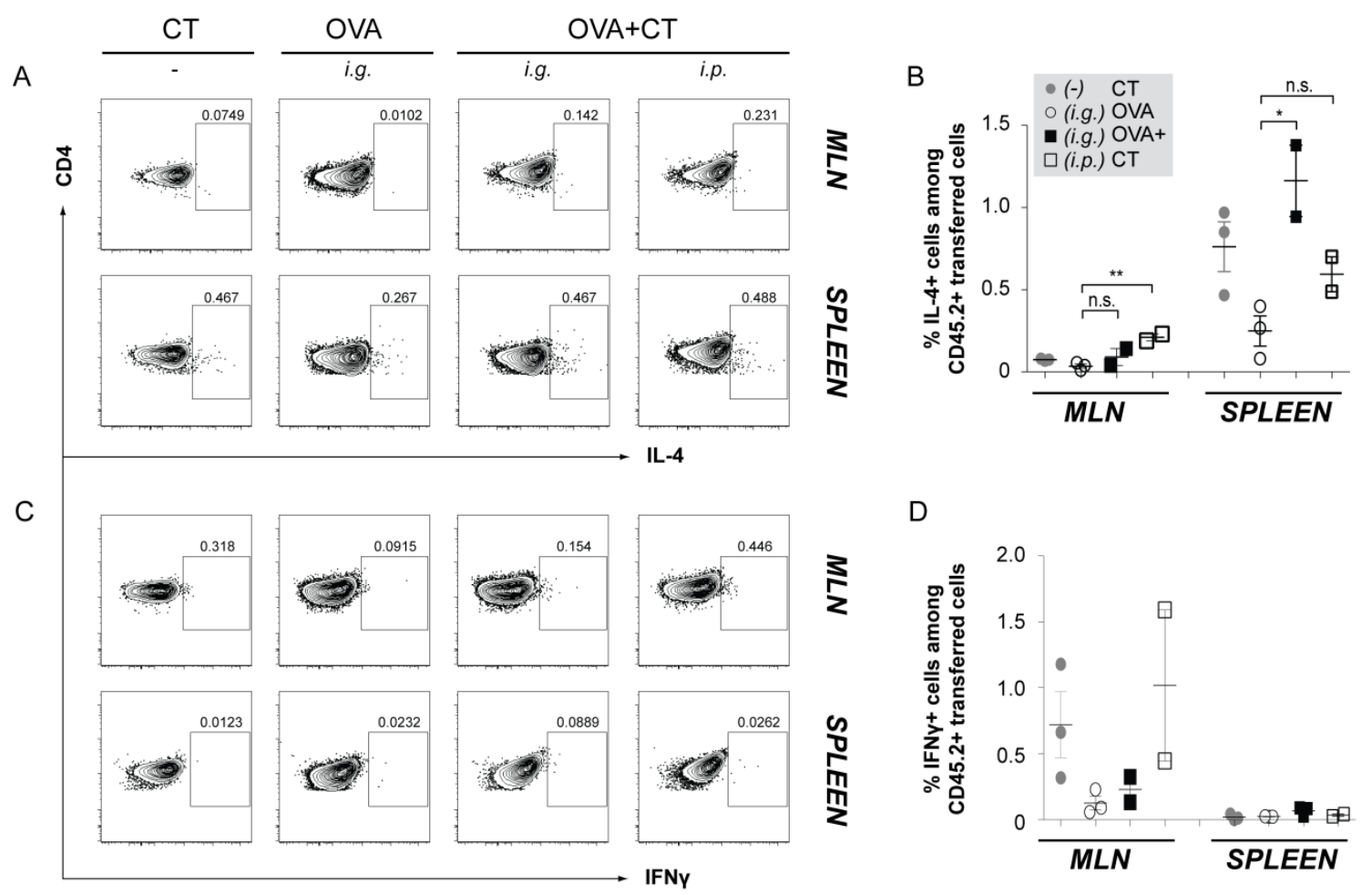

Figure 5.6: Antigen specific cytokine production could not be detected by ICS following oral OVA+CT treatment

B6 congenic mice were injected i.v. with $5 \times 10^{6}$ CD 45.2+ OTII splenocytes enriched by magnetic selection. One day later mice were treated with $10 \mu \mathrm{g}$ CT or $50 \mathrm{mg}$ OVA or a combination by oral gavage. On day 6, mice primed with OVA were given a second dose of OVA by oral gavage $(50 \mathrm{mg})$ or by intraperitoneal injection $(100 \mu \mathrm{g})$. On day 7 , mice were injected with $250 \mu \mathrm{g}$ brefeldin A i.v. to prevent release of cytokine from the cell. Four hours later, T cells were isolated from the MLN and spleen and assessed for cytokine production by intracellular staining. Representative contour plots show assessment of IL-4 (A) and IFN $\gamma$ (C) production. Frequency of transferred cells producing IL4 (B) anf IFN $\gamma$ (D) are shown on graphs. Graphs show mean \pm SEM. Statistical significance was determined by Two-Way ANOVA with Bonferroni post-test. * $\mathrm{p}<0.05 * * 0.001<\mathrm{p}<0.05$ 


\subsubsection{RALDH activity does not change following oral administration of CT}

Activity of RALDH is required for $\mathrm{CD} 103+\mathrm{DC}$ to metabolise RA from vitamin A derivatives. At steady-state, RA production by intestinal DC is important for promoting the differentiation of Tregs and maintaining tolerance. Following CT treatment, Treg numbers have been shown to decrease and effector $\mathrm{T}$ cell numbers increase ${ }^{60}$. Therefore, it was of interest to compare CD103+ DC RALDH activity between steady-state and following CT treatment using the flow cytometry ALDEFLUOR ${ }^{\mathrm{TM}}$ assay. I found CT treatment did not affect RALDH activity as approximately $75 \%$ of CD103+ CD11b+ DC from the LP of naïve and OVA+CT treated mice expressed RALDH (Fig. 5.7, A). Furthermore, in the MLN, a similar frequency of RALDH+ CD103+ CD11b- and CD103+ CD11b+ DC (50$55 \%$ and 60-65\% respectively) were seen from naïve and OVA+CT treated mice (Fig.5.7, B). This data suggests that CT treatment does not affect the activity of RALDH in intestinal DC. 

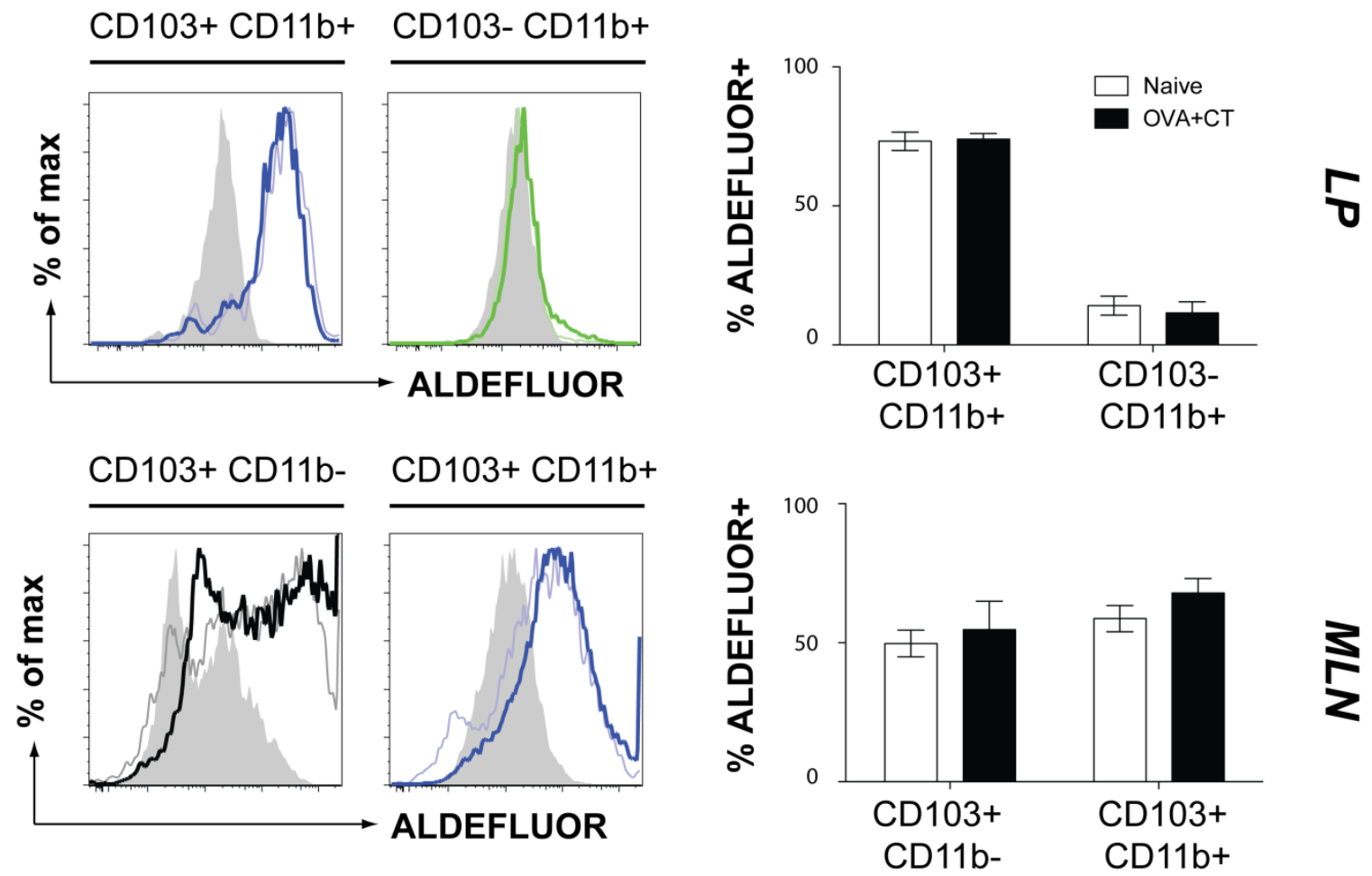

Figure 5.7: CT treatment does not change RALDH activity among intestinal APC populations

APC from the LP and MLN were isolated 17 hours following intragastric OVA+CT. RALDH activity was determined using the ALDEFLUOR staining kit and is shown for each subset from naïve (pale line) and OVA+CT treated (bold line) mice compared to cells treated with RALDH inhibitor diethylaminobenzlaldehyde (DEAB) (shaded). Bar graph shows percent RALDH positive for each APC subset from LP (A) and MLN (B). Data was pooled from two independent experiments. Graph showed mean \pm SEM. 


\subsubsection{CD103+ TIM-4+ DC show an increased capacity to take up apoptotic cells} compared to CD103+ TIM-4- DC ex vivo

TIM-4 has been shown to bind PtdSer and assists with the uptake of apoptotic cells ${ }^{187,}{ }^{188}$. Therefore, I wanted to determine if TIM-4 expression on CD103+ DC in the LP and MLN from CT treated mice was associated with an increased capacity for these cells to take up apoptotic cells. To address this, cells were isolated from the LP and MLN 17 hours post $\mathrm{OVA}+\mathrm{CT}$ treatment and CD11c+ cells were enriched by positive magnetic selection using an AutoMACS system. LP and MLN preparations were enriched for populations of interest and to minimise background staining. Apoptotic cell uptake was measured in CD11c+ cells that were co-cultured with e670 labelled apoptotic thymocytes. Staining of thymocytes with apoptotic cell markers Annexin-V and PI confirmed that $92 \%$ of thymocytes were undergoing apoptosis prior to co-culture (Fig. 5.8, A). CD11c+ APC were assessed for e670 expression by flow cytometry after one hour of culture. I found that live cells were enriched for FSC-A ${ }^{\text {hi }}$ SSC-A ${ }^{\text {hi }}$ CD11c + MHCII+ cells which indicates that the magnetic enrichment successfully isolated APC of interest. However, APC with low to intermediate expression of CD11c, such as CD103- TIM-4+ and CD103- TIM-4- cells, may not have been labelled with the magnetic beads to the same efficiency as $\mathrm{CD} 11 \mathrm{c}^{\text {hi }}$ expressing populations as the enrichment had a disproportionately high number of CD103+ cells compared to CD103(Fig. 5.8, B). Assessment of individual APC subsets from the LP showed approximately 85\% of CD103+ TIM-4+ DC acquired apoptotic thymocytes compared to 60\% of CD103+ TIM-4- DC (Fig. 5.9, A). Although not statistically significant, approximately $95 \%$ of CD103+ TIM-4+ DC from MLN acquired apoptotic thymocytes compared to 55\% of CD103+ TIM-4- DC (Fig. 5.9, B). Furthermore, approximately 95\% of CD103- TIM-4+ $\mathrm{M} \varphi$ and 70\% of CD103- TIM-4- cells from the LP acquired apoptotic material following coculture whereas only 15-20\% of CD11b- DC from the MLN acquired apoptotic material. In summary, this data suggests that expression of TIM-4 on DC allows an increased frequency of DC to acquire apoptotic material ex vivo. 
A

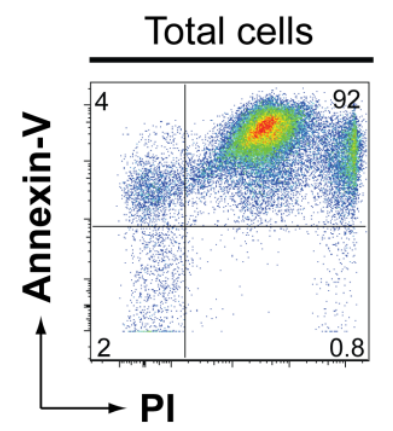

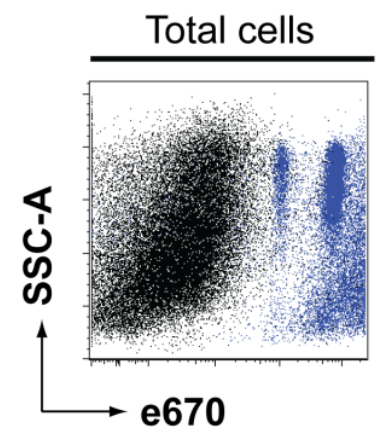

unlabelled thymocytes

labelled thymocytes

B

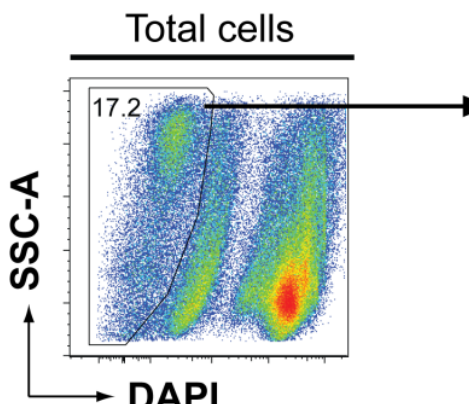

DAPI
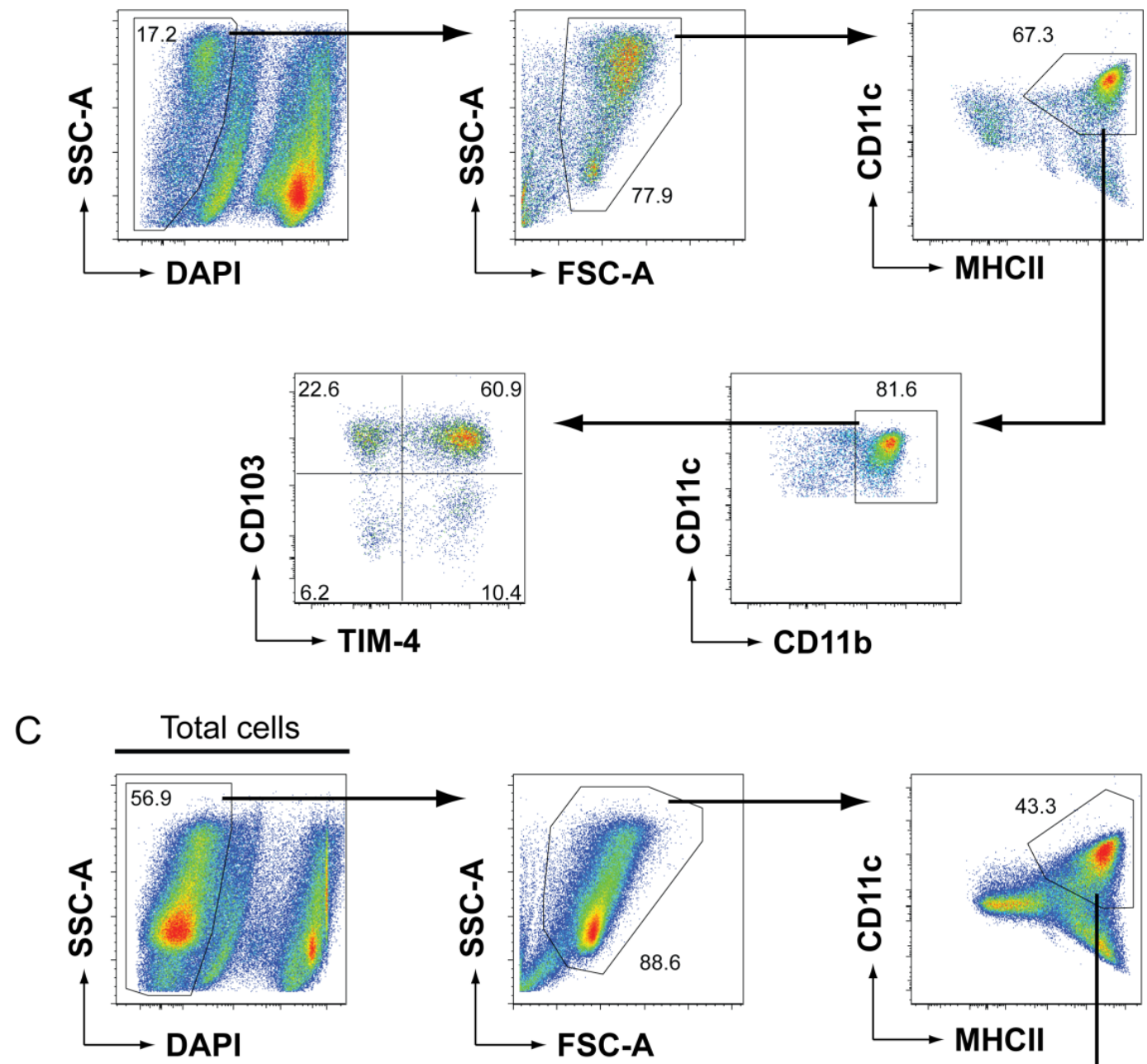

FSC-A
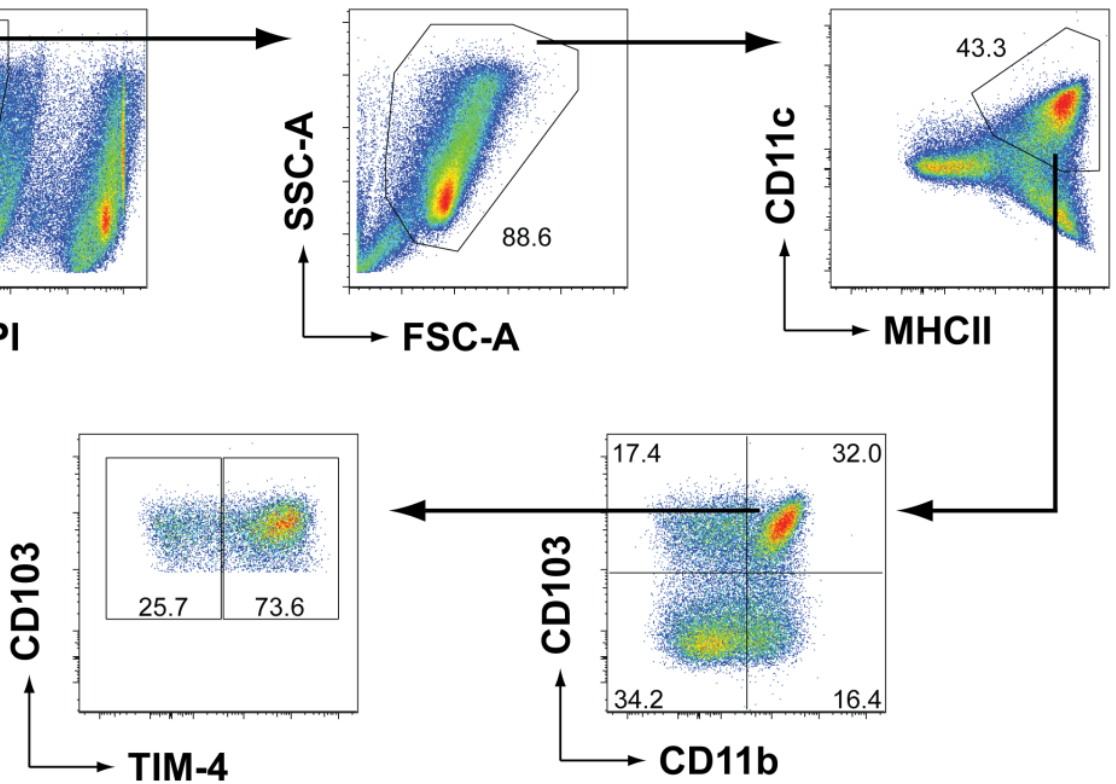
Figure 5.8: Dexamethasone induces apoptosis in thymocytes and CD11c+ positive magnetic enrichment preferentially enriches for CD103+ DC in the LP and MLN

Thymocytes from 4 week old mice were processed into a single cell suspension and labelled with proliferation dye e670. Apoptosis was induced by incubating thymocytes in $10 \mu \mathrm{M}$ dexamethsone for 4 hours, after which apoptosis was confirmed by Annexin-V and PI staining (A). Cells isolated from the LP and MLN of OVA+CT treated mice were enriched for CD11c+ cells by positive magnetic selection and co-cultured with e670 labelled apoptotic thymocytes. Dot plots show gating strategy for assessing individual APC populations for e670 fluorescence from the LP (B) and MLN (C).

A

LAMINA PROPRIA
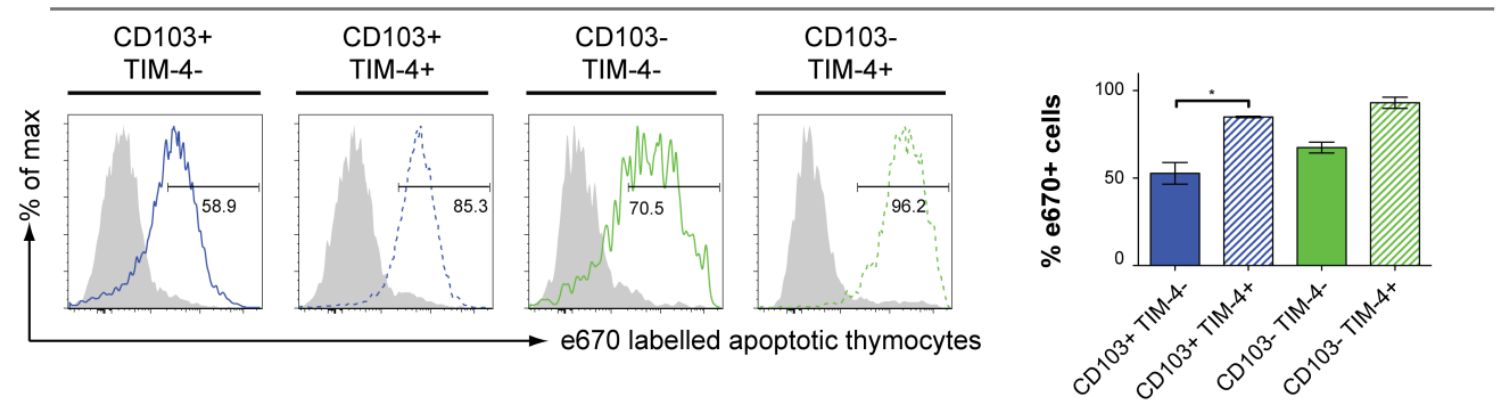

B

MESENTERIC LYMPH NODE
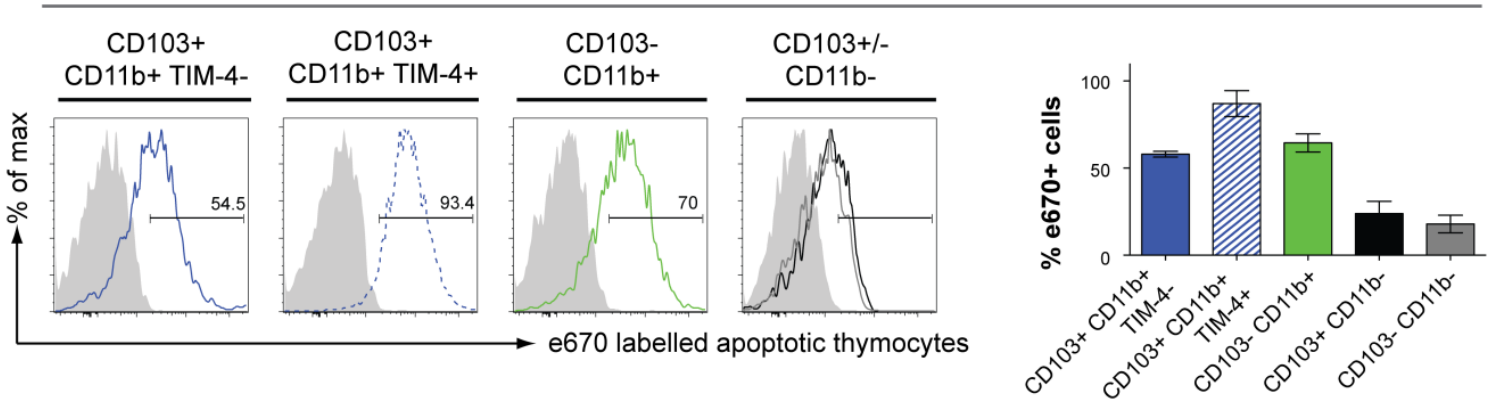

Figure 5.9: CD103+ TIM-4+ show an increased capacity for apoptotic cell uptake compared to CD103+ TIM-4- DC.

Apoptosis was induced in thymocytes labelled with e670 dye by incubating in $10 \mu \mathrm{M}$ dexamethasone for 4 hours. CD11c + cells isolated from OVA + CT treated mice were enriched by magnetic selection and cultured for 1 hour at 1:50 ratio with labeled apoptotic thymocytes. Apoptotic cell uptake capacity was determined by flow cytometry. Representative histograms show apoptotic cell uptake by DC subsets from the LP (A) and MLN (B). Data pooled from two individual experiments is shown on bar graphs. Graphs show mean \pm SEM. Statistical significance was determined by One-Way ANOVA with Bonferroni post-test. * $\mathrm{p}<0.05$ 


\subsubsection{TIM-4 upregulation by $\mathrm{CD} 103+\mathrm{DC}$ modestly increases antigen uptake capacity}

To date, antigen uptake by CD103+ DC in the LP has not been detected during steady-state

${ }^{56}$, however, it is unclear whether these cells are able to acquire antigen following maturation. CD103+ TIM-4+ DC showed an increased capacity for apoptotic cell uptake, therefore, I hypothesised that LP CD103+ TIM-4+ DC may be more efficient at the uptake of orally administered antigen compared to CD103+ TIM-4- DC. To address this question, mice were pre-treated with CT by oral gavage to induce TIM-4 upregulation on CD103+ DC. At the peak accumulation of CD103+ TIM-4+ cells (17 hours), mice were fed OVA labelled with the fluorescent dye AF647 (OVA-AF647) and sacrificed one hour later to visualise antigen uptake by LP cells. The data shows approximately $1.5 \%$ of CD103+ TIM-4+ DC acquired AF647 fluorescence compared to $0.8 \%$ of CD103+ TIM-4- DC (Fig. 5.10). Furthermore, as an indication of the quantity of antigen within the cell, I assessed the AF647 MFI among AF647+ APC. On average, I found CD103+ TIM-4+ cells had an AF647 MFI of 300 whereas CD103+ TIM-4- cells had an AF647 MFI of 100. Interestingly, CD103TIM-4+ M $\varphi$ had an AF647 MFI of approximately 450 compared to CD103- TIM-4- cells which had an AF647 MFI of 175. This data suggests that CD103+ DC that express TIM-4 in response to CT, have an increased capacity for antigen uptake compared to CD103+ TIM-4- DC. 
A

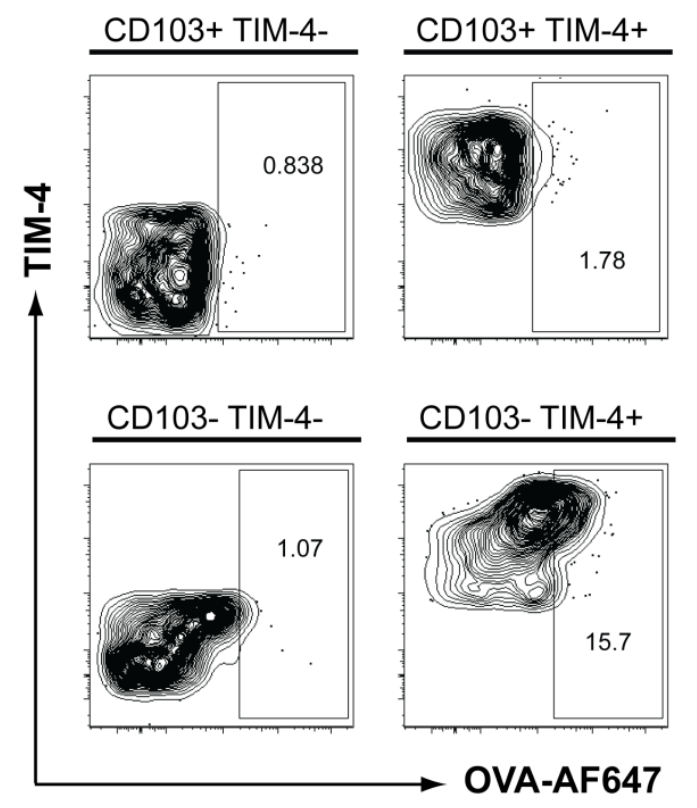

B

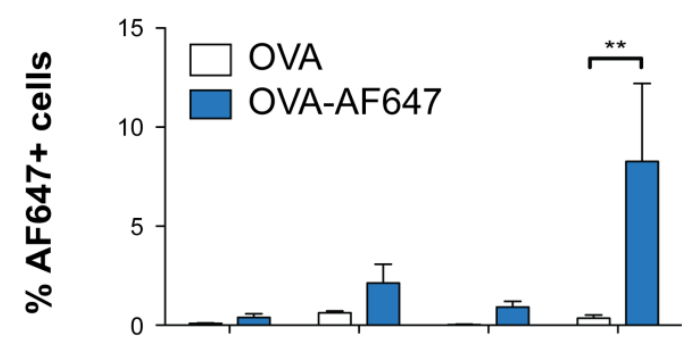

C

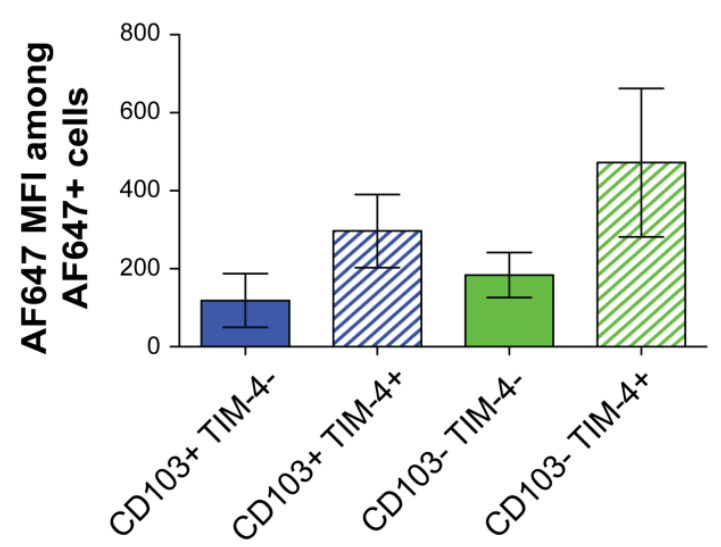

Figure 5.10: TIM-4 upregulation by CD103+ DC modestly increases antigen uptake capacity in vivo.

Mice were pre-treated with $10 \mu \mathrm{g}$ CT in bicarbonate buffer by oral gavage to induce TIM-4 upregulation. Mice were subsequently fed 50mg OVA or 250 $\mathrm{gg}$ OVA-AF647 in bicarbonate buffer 17 hours after initial CT treatment. LP cells were isolated one hour after OVA feeding and analysed for AF647 fluorescence by flow cytometry. Contour plots show AF647 fluorescence among DC subsets from the LP (A). Bar graph shows percent AF647+ cells among total CD11c+ MHCII+ cells from the LP (B) and AF647 MFI among AF647+ cells from each APC population (C). MFI value was calculated by subtracting average background fluorescence as determined from OVA treated mice. Graphs show mean \pm SEM. Statistical significance was determined by Two-Way ANOVA with Bonferroni post-test. ${ }^{* *} 0.001<\mathrm{p}<0.05$ 


\subsection{Discussion}

In this Chapter I show that co-administration of OVA antigen and CT promoted antigen specific $T$ cell expansion and production of the cytokine IL-4 and led to functional changes in intestinal CD103+ DC that express TIM-4 following CT treatment. I hypothesised that activation of antigen specific immune responses to OVA in the presence of CT may result from a functional change within APC subsets in the intestine. To investigate this hypothesis, I focussed on the CD103+ DC population which I showed to upregulate TIM-4 and CD86 following CT treatment. As TIM-4 is a receptor for PtdSer expressed on apoptotic cells and is required for apoptotic cell uptake ${ }^{187}$, I was interested to investigate the capacity of CD103+ TIM-4+ DC to firstly take up apoptotic cells and secondly to take up antigen. I found that CT treatment resulted in a modest increase in the capacity of CD103+ TIM-4+ DC to take up apoptotic cells ex vivo and antigen in vivo compared to CD103+ TIM-4- cells. In addition, as CD103+ DC have been shown to have a specialised ability to promote the differentiation of Tregs through their production of RA, it was of interest to determine whether this activity was altered after CT treatment. To investigate this, CD103+ DC were analysed for the expression of RALDH, an enzyme involved in RA metabolism. Interestingly, CT treatment did not result in a significant change in RALDH activity by CD103+ DC from steady-state. Although RALDH is well known for its role in RA production and the development of Tregs, it is possible that it does not alter the ability of DC to drive other immune responses. Overall, this data shows that oral administration of CT induces some functional changes among intestinal T cells and, specifically, CD103+ DC that express TIM-4. Therefore, I propose CD103+ TIM-4+ DC may be a suitable candidate for future study into the promotion of effector $\mathrm{T}$ cell responses following oral administration of CT.

\subsubsection{CT enhances antigen specific $\mathbf{T}$ cell proliferation}

Adjuvants such as CT, are used to potentiate immune responses to co-delivered antigen in vaccination settings. Therefore, I hypothesised that oral co-administration of OVA antigen 
with CT would enhance antigen specific $\mathrm{T}$ cell expansion. In line with this hypothesis, I found that oral administration of OVA $+\mathrm{CT}$ increased the frequency of antigen specific $\mathrm{T}$ cells present in the MLN compared to administration of antigen alone. As equal numbers of OTII cells were transferred at the start of the experiment, this result suggests that the OTII cells have proliferated more extensively in mice treated with OVA+CT compared to mice treated with OVA alone. Interestingly, OTII cells from OVA+CT and OVA treated mice had comparable expression of CD44 which indicates similar T cell antigen experience. This suggests CT was not required for OVA protein to be taken up by LP APC and presented to antigen specific $\mathrm{T}$ cells. Furthermore, mice treated with $\mathrm{CT}$ alone had significantly lower expression of $\mathrm{CD} 44$ compared to mice that received OVA. This observation supports the finding that CD44 expressed by OTII cells from OVA treated mice is indicative of antigen experience rather than an artefact of the adoptive transfer. Overall, these results suggest that OTII cells have similar opportunity to interact with antigen regardless of the presence of CT. However, CT has the capacity to enhance antigen specific T cell expansion in the MLN.

\subsubsection{CT increases the frequency of IL-4 producing $T$ cells in the intestine and spleen}

CT is commonly used as an adjuvant in models of food allergy because it has been shown to drive differentiation of naïve CD4 T cells to Th2 cells ${ }^{155,156,189}$. Therefore, I hypothesised that following oral administration of OVA+CT there would be an increase in the frequency of antigen specific T cells producing the Th2 cytokine IL-4 compared to Th1 cytokine IFN $\gamma$.

Importantly, for the detection of IL-4 production I had access to a transgenic mouse in which cells express the fluorescent reporter AmCyan when IL-4 message is transcribed, thus providing a powerful tool for the sensitive and accurate measurement of IL-4 producing cells. Using this technique I found that oral administration of OVA antigen and CT resulted in a significant increase in antigen specific T cells expressing AmCyan in the LP, MLN and spleen. 
Since I did not have access to IFN $\gamma$ reporter mice, I assessed $\mathrm{T}$ cell production of this cytokine by ICS after in vivo treatment with brefeldin A. However, I could not detect production of this cytokine above background staining. Likewise, assessment of IL-4 by this method yielded no detectable increase above background staining. IL-4 protein is quickly degraded after release whereas signal from a fluorescent reporter can be detectable long after transcription ${ }^{190}$. Thus, in this context, data from IL-4 reporter mice is likely to be more sensitive than ICS. Overall, from these studies, it is not clear whether there is antigen specific $\mathrm{T}$ cell production of IFN $\gamma$ however, results from reporter mice suggest that orally administered CT can induce antigen specific T cell production of IL-4.

\subsubsection{CT does not affect RALDH activity among LP and MLN APC}

Initiation of immune responses in the intestine has been associated with a corresponding decrease in activity of the RA metabolising enzyme RALDH among CD103+ DC. RA plays a key role in promoting differentiation of Tregs at steady-state, therefore, it has been suggested that loss of RALDH activity may be involved in the initiation of effector T cell responses ${ }^{175}$. In Chapter 4, I showed CD103+ TIM-4- and CD103+ TIM-4+ DC had similar RALDH activity, indicating that DC that express TIM-4 did not have an impaired capacity for RA metabolism. As I observed antigen specific T cell expansion and IL-4 production as opposed to a tolerised response following oral OVA+CT, I hypothesised that there would be an overall decrease in the activity of RALDH among APC from the LP and MLN. However, in contrast to this hypothesis, I could not detect any overall difference in the activity of RALDH between CD103+ DC from the LP and MLN of naïve and CT treated mice. Therefore, this data suggests that APC RALDH activity does not impair the ability of APC to drive effector $\mathrm{T}$ cell responses after $\mathrm{CT}$ treatment. 


\subsubsection{CD103+ TIM-4+ DC have an increased capacity for apoptotic cell uptake ex vivo compared to CD103+ TIM-4- DC}

Accumulating evidence has shown TIM-4 binds PtdSer on the surface of apoptotic cells and assists with their capture and uptake ${ }^{187,} 188$. Therefore, I hypothesised that CD103+ TIM-4+ DC that are detected following oral administration of CT will have an increased capacity for apoptotic cell uptake compared to CD103+ TIM-4- DC. In support of this, I found, using an in vitro phagocytosis assay, that a higher frequency of CD103+ TIM-4+ DC from the LP and MLN took up apoptotic material compared to CD103+ TIM-4- DC. Although, all CD11c + cells showed some capacity for apoptotic cell capture, this was most likely due to the large excess of apoptotic cells added to the culture. Once cells begin to undergo apoptosis they clump and become difficult to count, therefore, more apoptotic cells were possibly added to the co-culture with DC than what was required. Overall, expression of TIM-4 by CD103+ DC appears to increase their ability to take up apoptotic cells ex vivo.

\subsubsection{CD103+ TIM-4+ DC have a modest increase in capacity for antigen uptake in vivo compared to CD103+ TIM-4- DC}

Immune responses to oral antigen requires antigen uptake by APC in the LP, followed by the transport of antigen to the draining lymph node ${ }^{111}$. Interestingly previous studies have only detected antigen within the $\mathrm{CX}_{3} \mathrm{CR} 1+\mathrm{APC}$ population, which are not thought to migrate to the draining lymph node ${ }^{42,56}$. I hypothesised that CT treatment may alter the ability of CD103+ DC, in particular the more activated CD103+ TIM-4+ DC, to take up antigen. To investigate this, I measured expression of fluorescent OVA (OVA-AF647) among APC subsets in the LP. In support of my hypothesis, my data showed a small frequency of CD103+ TIM-4+ DC expressing OVA-AF647 whereas CD103+ TIM-4- DC did not show any expression of OVA-AF647. Overall, this data suggests that CD103+ DC that express TIM-4 after CT treatment may gain some capacity to take up luminal antigen in vivo. 


\subsection{Conclusions}

In this Chapter I show that in addition to altering CD103+ DC phenotype CT induces functional changes in this population. While I found no change in RALDH activity, CD103+ DC that express TIM-4 were more effective at apoptotic cell uptake ex vivo and acquired a modest capacity for antigen uptake in vivo. Thus, expression of TIM-4 appears to be associated with different functional capacities to CD103+ TIM-4- DC. Furthermore, I found CT promoted the expansion of antigen specific T cells and led to a Th2 immune response in the intestine which was characterised by an increase in the frequency of IL-4 producing $\mathrm{T}$ cells. Therefore, alterations in DC phenotype and function may contribute to the development of immunity to CT in the intestine. 


\section{Chapter 6: General Discussion}




\subsection{Summary of findings}

In this thesis I show a novel method of identifying DC and $\mathrm{M} \varphi$ populations from the jejunal LP and MLN at steady-state. Secondly, using this method I identified a proportion of jejunal LP APC that alter their morphology, phenotype and function following oral administration of the mucosal adjuvant CT. This population can be identified by co-expression of cell surface markers CD103 and TIM-4 and shows elevated expression of co-stimulatory molecule CD86. Thirdly, I found CD103+ TIM-4+ DC to have an increased capacity for apoptotic cell uptake and some increased ability to take up antigen, suggesting that these cells are somewhat functionally distinct from steady-state CD103+ DC. In addition, it appears CD103+ DC that upregulate TIM-4 maintain their capacity to migrate to the MLN and thus may contribute to the generation of $\mathrm{T}$ cell responses. Lastly, $\mathrm{I}$ found oral administration of OVA $+\mathrm{CT}$ to be associated with an increase in frequency of antigen specific IL-4 producing T cells in the intestine and spleen. Therefore, I propose CD103+ TIM-4+ DC may be contributing to the adjuvant activity of CT, although, the mechanisms which lead to the appearance of this population and the mechanisms by which these DC alter immune function is yet to be explored. Understanding how CD103+ TIM-4+ DC contribute to the development of intestinal immunity will provide insight into the mechanisms that lead to the development of effector responses. 


\subsection{Discussion}

\subsubsection{Phenotype of intestinal APC}

Intestinal APC identified by expression of CD11c and MHCII consist of a mixture of DC and $M \varphi$. Intestinal APC have been broadly classified into two populations: CD103+ and $\mathrm{CX}_{3} \mathrm{CR} 1+$, which represent DC and $\mathrm{M} \varphi$ populations respectively (see Section 1.3) ${ }^{42,101}$. In this thesis I highlighted the heterogeneity of intestinal APC populations which cannot be appreciated using the current classification system. Recent reviews support this interpretation, with a number of authors emphasising the complexity of intestinal APC populations and oversimplification of the phenotypic characterisations used in many studies 191,192

In my studies I focussed on $\mathrm{CD} 11 \mathrm{~b}+\mathrm{APC}$ which make up the majority of $\mathrm{CD} 11 \mathrm{c}+$ MHCII+ cells in the jejunal LP. At steady-state, I found CD11b+ APC can be grouped into three distinct populations by assessing the cell surface markers CD103 and TIM-4. Specifically these were CD103+ TIM-4-, CD103- TIM-4- and CD103- TIM-4+ (see Section 3.3.2). Interestingly, following oral administration of CT, I observed a fourth population of CD11b+ APC that co-express CD103 and TIM-4 (see Section 4.3.1.). I concluded from further phenotypic study that CD103+ TIM-4- DC closely resembled the previously characterised CD103+ DC which promote Treg differentiation through production of RA ${ }^{57}$, 58. Similar to published reports I found CD103+ TIM-4- DC to lack expression of M $\varphi$ markers $\mathrm{F} 4 / 80^{42}, \mathrm{CX}_{3} \mathrm{CR} 1{ }^{42,101}$ and $\mathrm{CD} 64^{113}$ and have high activity of the RA metabolising enzyme RALDH ${ }^{57,58}$. Importantly, I found the CD103+ TIM-4+ cells that appeared following CT treatment to share these characteristics. This strongly suggests that a proportion of CD103+ DC upregulate TIM-4 in response to CT (see Section 4.4.1.). This finding highlights the highly plastic nature of intestinal DC and the fact immunostimulatory agents can alter their phenotype.

Furthermore, I found CD103- TIM-4+ cells to represent the intestinal $\mathrm{M} \varphi$ population that has been previously identified by their expression of $\mathrm{CX}_{3}$ CR1. CD103- TIM-4+ cells had a 
vacuolar cytoplasm that has been shown to be characteristic of $\mathrm{CX}_{3} \mathrm{CR} 1+$ cells ${ }^{101}$. Also, I found CD103- TIM-4+ cells to express M $\varphi$ markers F4/80 ${ }^{42}, \mathrm{CX}_{3} \mathrm{CR} 1{ }^{42,101}$ and CD64 ${ }^{113}$ and lacked RALDH ${ }^{57,58}$ activity which is corresponds to the phenotypic descriptions in published reports.

In addition to CD103+ TIM-4- and CD103- TIM-4+ populations, I identified a population of cells that expressed neither of these markers but still expressed CD11b (CD103- TIM-4-). Due to their lack of CD103 expression it is likely this population would have previously been included within the $\mathrm{CX}_{3} \mathrm{CR} 1+\mathrm{M} \varphi$ population. I found CD103- TIM-4- cells to consist of both DC and $\mathrm{M} \varphi$ as there was varying expression of F4/80, CX 3 CR1, CD64 and RALDH (see Section 3.4.1). These findings are supported by a recent paper which showed a proportion of $\mathrm{CX}_{3} \mathrm{CR} 1+$ cells to express the DC specific protein zBTB46 ${ }^{103}$. Furthermore, a small population of $\mathrm{CX}_{3} \mathrm{CR} 1^{\text {int }}$, which are derived from blood monocytes ${ }^{99}$, have been reported in the LP at steady-state ${ }^{42}$. It is possible that a constituent of the CD103- TIM-4population includes these $\mathrm{CX}_{3} \mathrm{CR} 1^{\text {int }}$ cells.

Finally, in terms of translational applications, it is important to note that APC populations described in mice are similar, phenotypically and functionally, to APC populations described in humans (see Table 2) ${ }^{109,113,193}$. A recent study provided significant support for this finding by showing a comprehensive transcriptional and functional comparison between jejunal LP DC from mice and humans ${ }^{193}$. As in mice, the predominant DC population found in the jejunal LP at steady-state are CD103+CD11b+. Due to the highly conserved phenotype and functions of intestinal APC between mice and humans, the findings reported in this thesis such as the changes in phenotype, function and morphology to CD103+ CD11b+ DC upon stimulation with CT are relevant to understanding how intestinal immune responses are initiated in humans. 
Table 2: Summary of the phenotype and function of intestinal APC from mice and humans

\begin{tabular}{|c|c|c|c|}
\hline $\begin{array}{l}\text { Cell } \\
\text { type }\end{array}$ & $\begin{array}{c}\text { Mouse } \\
\text { phenotype }{ }^{42,101}\end{array}$ & $\begin{array}{c}\text { Human } \\
\text { phenotype } 193\end{array}$ & Functional characteristics \\
\hline $\mathrm{DC}$ & $\begin{array}{l}\text { CD103+ } \\
\text { CX }{ }_{3} \text { CR1- } \\
\text { CD11b- }\end{array}$ & $\begin{array}{l}\text { CD103+ } \\
\text { CX }{ }_{3} \text { CR1- } \\
\text { CD11b- } \\
\text { Sirp } \alpha-\end{array}$ & $\begin{array}{l}\text { Migratory capacity }(\mathrm{CCR} 7+) \text { in mice } 111 \\
\text { and humans }{ }^{193} \text {. } \\
\text { Promote gut tropism in mice }{ }^{81} \text { and } \\
\text { humans } 109,193 \text {. } \\
\text { Promote Treg differentiation in mice }{ }^{81} \text {. } \\
\text { Promote Th17 differentiation in humans }{ }^{193} \text {. }\end{array}$ \\
\hline DC & $\begin{array}{l}\text { CD103+ } \\
\text { CX }_{3} \text { CR1- } \\
\text { CD11b+ }\end{array}$ & $\begin{array}{l}\text { CD103+ } \\
\text { CX }_{3} \text { CR1- } \\
\text { CD11b+ } \\
\text { Sirp } \alpha+\end{array}$ & $\begin{array}{l}\text { Migratory capacity }(\mathrm{CCR} 7+) \text { in mice } 111 \\
\text { and humans }{ }^{193} \text {. } \\
\text { Have high RALDH activity and promote } \\
\text { Treg differentiation and gut tropism in } \\
\text { mice }{ }^{57,58,81} \text { and humans }{ }^{109,193} \text {. } \\
\text { Promote Th17 differentiation in mice }{ }^{81} \text {, } \\
{ }^{108} \text { and humans }{ }^{193} \text {. }\end{array}$ \\
\hline moDC & $\begin{array}{l}\mathrm{CD}_{103-} \\
\mathrm{CX}_{3} \mathrm{CR} 1^{\mathrm{int}} \\
\mathrm{CD} 11 \mathrm{~b}+\end{array}$ & $\begin{array}{l}\text { CD103- } \\
\text { CX }_{3} \mathrm{CR}^{\text {int }} \\
\text { CD11b+ } \\
\text { Sirp } \alpha+\end{array}$ & $\begin{array}{l}\text { Migratory capacity (CCR7+) in mice }{ }^{99} \\
\text { and humans }{ }^{193} \text {. } \\
\text { Promote Th1 differentiation in mice }{ }^{114} \\
\text { and humans }{ }^{193} \text {. } \\
\text { Have high RALDH activity and promote Treg } \\
\text { differentiation in humans }{ }^{193} \text {. } \\
\text { Increase in frequency during } \\
\text { inflammation in mice }{ }^{114} \text { and humans }{ }^{193} \text {. }\end{array}$ \\
\hline $\mathrm{M} \varphi$ & $\begin{array}{l}\text { CD103- } \\
\text { CX }_{3} \text { CR1 } \\
\text { CDi } 11 b+\end{array}$ & $\begin{array}{l}\text { CD103- } \\
\mathrm{CX}_{3} \mathrm{CR}^{\mathrm{hi}} \\
\mathrm{CD} 14+ \\
(\mathrm{CD} 64+?)^{113}\end{array}$ & $\begin{array}{l}\text { Antigen capture in mice } 42,56,75 \text {. } \\
\text { Maintain tolerogenic environment } \\
\text { through production of IL-10 in mice }{ }^{60} \text {. } \\
\text { Function in humans requires further } \\
\text { investigation. }\end{array}$ \\
\hline
\end{tabular}

Italics indicate functional differences between mice and humans. 


\subsubsection{The direct and indirect effects of CT on APC phenotype and function}

CT is a potent mucosal adjuvant that is thought to drive effector $\mathrm{T}$ cell responses by

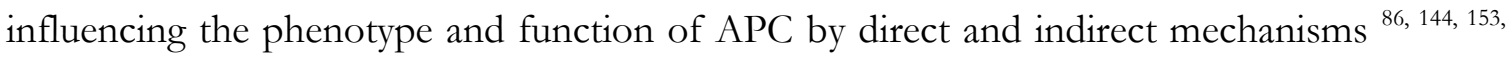
158, 159, 160, 161 . However, it is not clear whether oral administration of CT influences intestinal APC phenotype by direct or indirect interactions to promote $T$ cell expansion and cytokine production in the MLN. Therefore, it is of interest to understand the mechanisms by which CT influences the upregulation of TIM-4 and CD86 on CD103+ DC in the LP.

In order to have a direct effect on DC CT would need to bind specifically to a receptor on the cell surface. One published report suggested CT induced DC maturation and TIM-4 upregulation through TLR4 signalling in vitro. In this report, DC from CT and antigen treated TLR4-/- mice were unable to induce anaphylactic responses when adoptively transferred into naïve wildtype mice whereas CT primed DC from wildtype mice could ${ }^{144}$. Conversely, $\mathrm{C} 3 \mathrm{H} / \mathrm{HeJ}$ mice, which have a natural defect in TLR4 signalling ${ }^{194}$, develop anaphylaxis in response to antigen co-delivered with $\mathrm{CT}^{89,195,196}$. Thus, the requirement for TLR4 by DC to respond to CT is not clear. In my study, the expression of TIM-4 and CD86 by CD103+ DC following oral administration of CT was comparable in TLR4-/- and wildtype controls. This suggests that CT does not directly interact with DC through TLR4 in my model (see Section 4.4.2).

CT may act directly on intestinal DC via receptors other than TLR4 such as GM-1 (Fig. 6.1.). CT is known to exerts its effects on epithelial cells by binding to surface ganglioside receptors (GM-1) ${ }^{197}$ and gaining entry to the cellular cytoplasm ${ }^{150,151,152,153}$. Therefore, it is important to note that BMDC derived from GM-1 deficient mice maintained the capacity to mature to LPS but failed to mature in the presence of CT ${ }^{161}$. Furthermore, GM-1 expression is required for the adjuvant activity of $\mathrm{CT}$ as GM-1 deficient mice were unable to mount immune responses to antigen co-delivered with $\mathrm{CT}^{161}$. These studies suggest that the most likely direct target for CT on DC is GM-1 and not PRRs. In further support of this hypothesis, a recent study utilising bone marrow chimera mice which lack GM-1 only on 
haematopoietic cells shows that GM-1 expression on DC is required for DC maturation in response to $\mathrm{CT}$ and the induction of intestinal immunity to antigen co-delivered with $\mathrm{CT}{ }^{160}$.

Alternatively, CT may influence the phenotype and function of CD103+ DC indirectly by one of two mechanisms (Fig. 6.1.). Firstly, CT is known to cause epithelial barrier dysfunction ${ }^{177}$ which allows for commensals to populate the LP where they can interact with APC through TLRs and other PRRs. In Chapter 4, I show depletion of commensals with antibiotics did not prevent upregulation of TIM-4 and CD86 by CD103+ DC. Furthermore, as mentioned above, TLR4 deficiency did not affect the ability of CD103+ DC to upregulate TIM-4 in response to CT. Therefore, I concluded that CT induced barrier dysfunction was not involved in altering DC phenotype following CT treatment (see Section 4.4.2). Secondly, CT may be influencing other cells to produce cytokines which affect DC phenotype and function. As intestinal epithelial cells are the primary target for CT 150, 151, 152 and act as

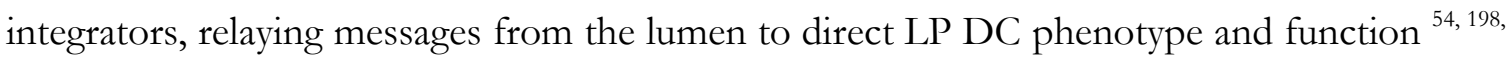
${ }^{199}$, it is likely they would be involved in any cytokine production induced by CT. CT treatment has been associated with activation of MAP kinases and NFxB in epithelial cells ${ }^{200}$ and the production of pro-inflammatory cytokines such as IL-6 ${ }^{201}$, IL-8, TSLP, TNF $\alpha$ and GM-CSF ${ }^{202,203}$ and the chemokines CCL2 and CCL20. An in vitro culture system showed that production of these chemical mediators by CT treated epithelial cells induced DC recruitment and maturation ${ }^{202}$. Thus, it is of interest to further investigate epithelial cytokines and the effects they have on intestinal DC.

In addition to epithelial cells, other innate immune cells such as eosinophils ${ }^{204}$, mast cells ${ }^{205}$ and innate lymphoid cells (ILC) ${ }^{205,206}$ provide vital signals that contribute to intestinal immunity. While these cells have not been directly associated with CT treatment, they have been shown to contribute to the development of intestinal immune responses through production of cytokines and chemokines in other settings. Therefore, these innate immune cells may play a role in promoting DC to drive immunity in the intestine following CT treatment. Intestinal eosinophils are present throughout the LP at steady-state ${ }^{207}$ and accumulate following inflammation, contributing to tissue damage in food allergy ${ }^{208}$ and 
IBD ${ }^{209}$. In addition to their own effector functions, it has been suggested eosinophils may affect intestinal DC responses through production of a vast array of cytokines and chemokines ${ }^{204}$ such as eosinophil-derived neurotoxin which has been shown to induce maturation and priming of DC to drive antigen specific Th2 responses ${ }^{210}$. Intestinal mast cells also accumulate during intestinal inflammation and produce pro-inflammatory cytokines IL-1 $\beta$, IL-6 and TNF $\alpha$ in response to danger signals from dying cells and activated monocytes ${ }^{211}$. Furthermore, recent studies have strongly implicated ILC in a number of immune processes in the intestine. A subset of ILC, known as ILC3, has been reported to be an important source of GM-CSF ${ }^{212}$ and IL-22 $213,214,215$ in the intestine. GM-CSF is a DC growth factor that is known to have profound effects differentiation of DC, DC activation and functional outcomes ${ }^{216,217,218}$. IL-22 assists with epithelial cell repair and tissue damage from infection as well as promoting production of pro-inflammatory cytokines and antimicrobial peptides by epithelial cells ${ }^{213,214,215}$.

\subsubsection{Functions of CD103+ CD11b+ DC in the LP and MLN}

There is evidence to suggest that CD103+ CD11b+ DC drive Treg and effector T cell responses under different conditions $57,58,60,73,103,108$. However, it is not clear whether these distinct immune responses instructed by the DC are mediated by specific subsets of CD103+ DC or whether factors from the environment condition DC to carry out specific functions. In this thesis I have demonstrated that the appearance of CD103+ TIM-4+ DC in the LP and MLN coincides with an increase in frequency of IL-4+ antigen specific T cells in the LP, MLN and spleen following oral administration of OVA+CT. I present data in Chapter 4 which suggests that CD103+ TIM-4+ are not a distinct population (see Section 4.4.1.) but rather represent a proportion of the CD103+ population that have responded to direct ${ }^{160}$ or indirect signals from CT ${ }^{202}$ (see Section 6.2.2.). Therefore, I propose that CD103+ TIM-4+ DC may be contributing to the development of effector responses to CT (Fig. 6.1). The mechanisms by which this is occurring requires further investigation but possible factors include changes in DC-T cell signalling via chemical mediators and receptorligand interactions and the presentation of apoptotic material. 


\section{Soluble mediator interactions between DC and T cells}

Importantly, despite the observed phenotypic changes, time course experiments suggest CD103+ TIM-4+ DC maintain their capacity to migrate to the MLN and thus have the opportunity to interact with T cells. T cell differentiation is primarily driven by cytokine and receptor mediated interactions between DC and T cells (see Section 1.1.2). Thus, changes in cytokine secretion and cell surface receptors on DC may result in altered T cell responses. DC exposed to CT have been shown to alter their cytokine profile with a number of reports showing CT to induce DC production of IL-1 $\beta^{151,202}$, IL-6, TNF $\alpha$, IL-23 ${ }^{202}$, RA and TGF $\beta$ ${ }^{219}$. Furthermore, CT has been shown to inhibit murine and human DC production of proinflammatory cytokines such as IL-12 and TNF $\alpha$ which is thought to promote the biased Th2 responses commonly associated with CT ${ }^{150,154,182,220}$. However, others have shown CT treated DC can lead to the generation of IL-4, IL-13 and IL-17 producing T cells ${ }^{202}$. Therefore, it would be of interest to assess the cytokine profile of CD103+ TIM-4+ cells compared to steady-state CD103+ TIM-4- cells to determine how CT affects DC cytokine profiles in this model.

Furthermore, due to the potential role CD103+ TIM-4+ DC have in inducing effector T cell responses it was of interest to consider the role of RA, the chemical mediator associated with driving the generation of tolerance promoting Tregs at steady-state. Of note, oral administration of CT has been associated with a decrease in the frequency of Tregs in the LP ${ }^{60}$ and decreased RA metabolism has been implicated in the development of effector immune responses ${ }^{175,212}$. However, I found steady-state CD103+ CD11b+ DC to have high activity of the RA metabolising enzyme RALDH which was maintained following oral CT treatment despite the generation of an antigen-specific T cell response. While RA is associated with preferentially promoting Treg differentiation in conjunction with TGF ${ }^{57,58}$ and inhibiting Th17 differentiation ${ }^{125,137}$, recent evidence suggests that RA may also be required for the induction of effector $\mathrm{T}$ cell responses by playing a role in the regulation of early $\mathrm{T}$ cell activation $^{221}$. In addition, RA is vital for upregulating gut homing receptors $\alpha 4 \beta 7$ and CCR9 
on $\mathrm{T}$ cells ${ }^{128,222,223}$. It is important that following activation by DC that $\mathrm{T}$ cells traffic from the lymph node to the tissue to exert their effector functions ${ }^{60}$. Therefore, as RA is involved in a number of aspects of the immune response, the activity of RALDH may need to be maintained and production of additional cytokines such as TGF $\beta$ and IL-6 may be the key factor in determining $\mathrm{T}$ cell phenotype.

Receptor mediated interactions between DC and T cells

In addition to cytokines and chemical mediators, receptor mediated interactions have been shown to play a role in determining T cell phenotype. In my study, I observed CD103+ DC to alter their phenotype by upregulating TIM-4 as well as an increase in the frequency of IL4 producing T cells in response to CT. Thus, it is possible that changes in DC cell surface markers ultimately lead to the generation of effector $\mathrm{T}$ cells that can produce IL-4. Therefore it is of interest that the interaction between TIM-4 on DC and TIM-1 on T cells has been implicated in driving Th2 responses in vitro and in vivo ${ }^{144,}{ }^{147}$. Blockade of TIM-4 with a monoclonal antibody prevented the induction of the Th2 response ${ }^{144}$ suggesting a role for TIM-4 in promoting Th2 differentiation. Therefore, investigation of $\mathrm{T}$ cell phenotype following culture with CD103+ TIM-4- and CD103+ TIM-4+ DC may provide an interesting avenue for future studies.

\section{Presentation of apoptotic material}

Another possible mechanism by which $\mathrm{CT}$ may affect $\mathrm{T}$ cell responses is through presentation of apoptotic material by DC. CD103+ TIM-4+ DC showed an increased capacity to take up apoptotic material ex vivo and may therefore present apoptotic material to T cells following migration to the MLN. For the most part, investigations into apoptotic cell presentation have been assessed at steady-state which is associated with development of tolerance and capture of apoptotic cells by DC has been shown to promote antiinflammatory responses including the induction of TGF $\beta$ production and inhibition of DC maturation ${ }^{224,225}$. In my model, CD103+ DC acquire TIM-4 expression following activation 
in response to an adjuvant which permits the uptake of apoptotic material. Adoptive transfer studies carried out in Chapter 5 show CT enhances expansion of antigen specific T cells to oral delivered antigen compared to antigen alone. Thus, it is unlikely uptake of apoptotic material by LPDC impairs the effector response. However, it is not known how acquisition of apoptotic material following activation would affect $\mathrm{T}$ cell differentiation. In vitro studies with human cells show that DC that have taken up apoptotic material in an immunostimulatory environment can drive Th1 responses by production of IL-12p70 ${ }^{226}$ which suggests that presentation of the apoptotic material by activated DC can drive effector $\mathrm{T}$ cell responses. Therefore, the ability for activated CD103+ DC to acquire apoptotic material prior to migration may influence $\mathrm{T}$ cell outcomes.

\subsubsection{Functions of monocyte-derived APC in the LP and MLN}

In addition to CD103+ cells which appear to be bona fide DC, there are populations of $\mathrm{CX}_{3} \mathrm{CR} 1+\mathrm{DC}$ and $\mathrm{M} \varphi$ found throughout the LP which develop from blood monocytes ${ }^{42,99}$. It has been suggested that environmental signals dictate the ratio at which $\mathrm{CX}_{3} \mathrm{CR} 1^{\text {int }}$ cells and $\mathrm{CX}_{3} \mathrm{CR} 1+\mathrm{M} \varphi$ are found in the LP, with inflammatory conditions favouring the $\mathrm{CX}_{3} \mathrm{CR} 1^{\text {int }}$ subset ${ }^{99,117}$. Despite these recent advances, the roles of $\mathrm{CX}_{3} \mathrm{CR} 1+$ cells in the development of intestinal effector responses have not been well defined.

Phenotypic studies presented in this thesis show CD103- TIM-4+ cells likely represent the reported $\mathrm{CX}_{3} \mathrm{CR} 1+\mathrm{M} \varphi$ population which have been shown to play an important role in the maintenance of Tregs in the LP through production of IL-10 ${ }^{60}$. Interestingly, I observed a decrease in expression of $\mathrm{CX}_{3} \mathrm{CR} 1$ on intestinal CD103- TIM-4+ $\mathrm{M} \varphi$ following CT treatment. As F4/80 and TIM-4 expression were maintained despite the decrease in expression of $\mathrm{CX}_{3} \mathrm{CR} 1$, it is unlikely that this observation is due to a change in ratio between $\mathrm{CX}_{3} \mathrm{CR} 1^{\text {int }} \mathrm{DC}$ and $\mathrm{CX}_{3} \mathrm{CR} 1+\mathrm{M} \varphi{ }^{99}$. However, the decrease in $\mathrm{CX}_{3} \mathrm{CR} 1$ expression is of interest because binding of $\mathrm{CX}_{3} \mathrm{CL} 1$ to $\mathrm{CX}_{3} \mathrm{CR} 1$ on the surface of $\mathrm{M} \varphi$ has been shown to regulate IL-10 production. Mice deficient in expression of $\mathrm{CX}_{3} \mathrm{CR} 1 \mathrm{had}$ reduced numbers of 
Tregs present in the LP compared to wildtype controls ${ }^{60}$. Therefore, this finding may indicate intestinal $M \varphi$ have a reduced capacity to produce IL-10 and thus favour a more immunostimulatory environment in the intestine following CT treatment.

Furthermore, the CD103- TIM-4- population that I detected in the LP appeared to be heterogeneous but did consist of some cells with lower expression of $\mathrm{CX}_{3} \mathrm{CR} 1, \mathrm{~F} 4 / 80$ and CD64. Therefore, the small number of $\mathrm{CX}_{3} \mathrm{CR} 1^{\text {int }}$ cells reported at steady-state ${ }^{42}$ may fall within this gate. I observed no significant change in the frequency of these cells following CT treatment, although, further in depth study is required to specifically assess whether $\mathrm{CX}_{3} \mathrm{CR} 1^{\text {int }}$ cells are involved in the generation of effector responses to CT. 


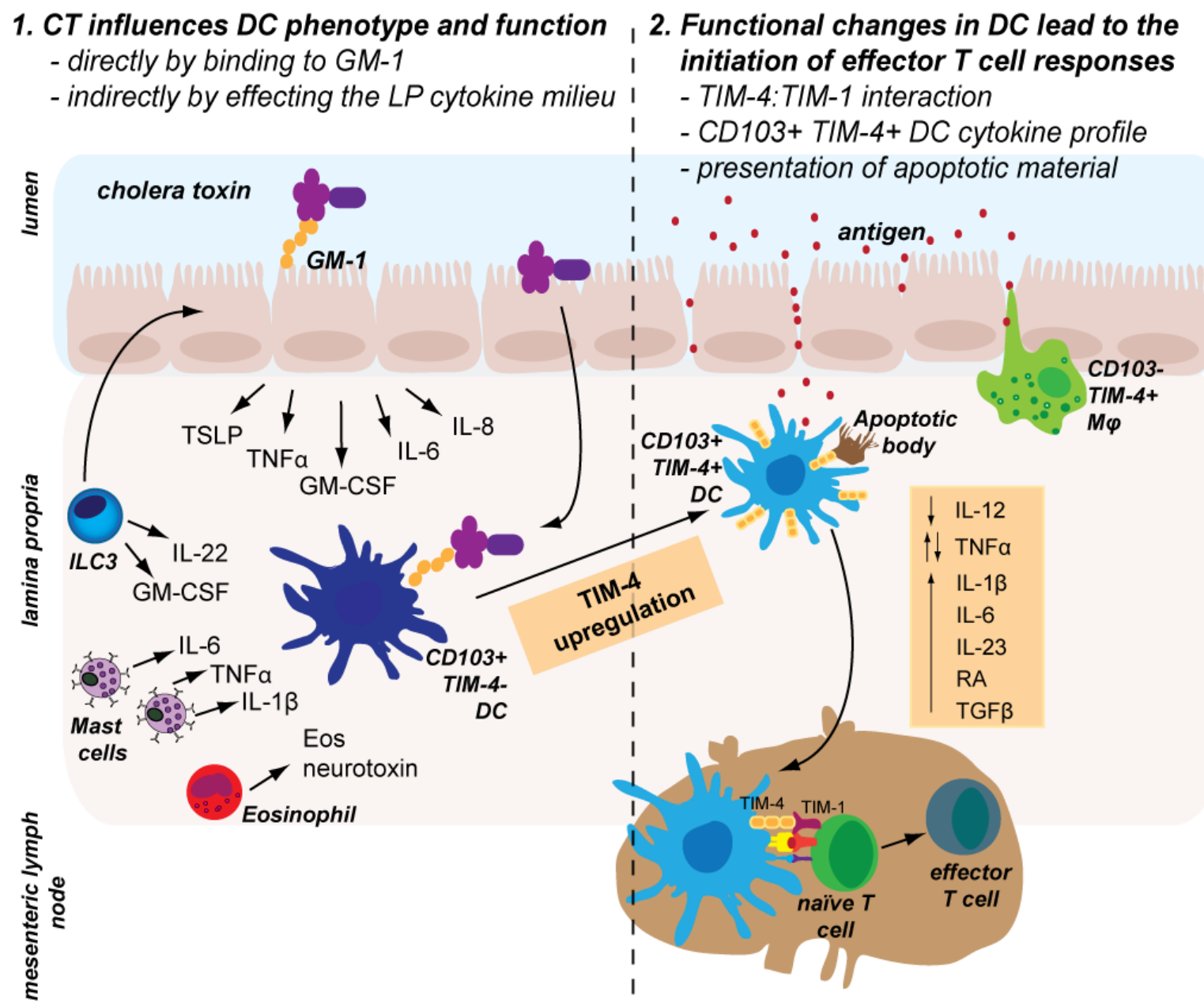

Figure 6.1: Proposed mechanisms by which CT treatment promotes phenotypic and functional changes among intestinal APC and T cells. 


\subsection{Future directions}

The intestine is a complex organ that is constantly exposed to foreign antigen. Therefore, strict regulation is at play to ensure appropriate immune responses are generated to specific antigens. The mechanisms by which immune responses are initiated in infection and disease are not well understood. My studies have associated the presence of CD103+ TIM-4+ DC in the LP and MLN with the initiation of effector Th2 responses in the intestine and spleen following oral administration of CT. However, it is unclear what signals lead to the upregulation of TIM-4 by CD103+ DC and whether or how CD103+ TIM-4+ DC contribute to the development of Th2 responses in the intestine. In order to gain a better understanding of these processes, I believe the following questions should be addressed:

a) What cytokines and cell types are being induced following CT administration?

b) How do these factors influence DC phenotype and function?

c) What APC subset/s and APC derived factors contribute to the initiation of intestinal immune responses?

I propose these future studies to investigate the effects of CT on intestinal APC phenotype and function:

1. Analysis of LP and MLN cytokine environments following oral administration of CT

At steady-state, intestinal M $\varphi$ secrete IL-10 to maintain Tregs in the intestine. Following oral administration of CT the frequency of LP Tregs has been found to decrease. In addition, loss of $\mathrm{CX}_{3} \mathrm{CR} 1$ signalling has been shown to decrease capacity of intestinal $\mathrm{M} \varphi$ to produce IL-10 in vivo ${ }^{60}$. Therefore, it would be of interest to assess IL-10 production capacity of CD103- TIM-4+ $\mathrm{M} \varphi$ which I observed to downregulate $\mathrm{CX}_{3} \mathrm{CR} 1$ after CT. This could be achieved by ICS following in vitro restimulation. 
Intestinal eosinophils, mast cells, ILC and epithelial cells can produce an array of cytokines in response to inflammatory stimuli which can influence DC priming and the subsequent $\mathrm{T}$ cell response. Therefore, I would like to investigate which cytokines are produced following oral CT administration. This can be achieved by intestinal punch biopsy Bioplex and ELISA analysis of cytokines such as GM-CSF, TSLP, IL-1 $\beta$, IL-6, IL-22, IL-25 and IL-33.

* CT treatment has been shown to alter DC cytokine profiles in vitro which can affect the capacity of DC to drive different $\mathrm{T}$ cell responses. Therefore, I would like to assess the capacity of intestinal DC to produce cytokine following oral administration of OVA. This could be achieved by ICS staining or ELISA analysis of DC culture supernatants.

2. Impact of cytokines on the function of DC subsets

The cytokine environment in the intestine can impact on the function of DC and thus affect $\mathrm{T}$ cell responses. Therefore, it would be interesting to assess the impact of different cytokines on the ability of intestinal DC to drive $\mathrm{T}$ cell proliferation and differentiation. Potential cytokine candidates could be identified in the experiments described above.

3. Flow cytometric analysis of innate immune cells present in the LP following oral administration of $C T$

Recent findings have implicated innate cells, other than DC and $\mathrm{M} \varphi$, in contributing to intestinal immunity, in part, through cytokine production. Changes in the intestinal cytokine environment may affect the phenotype and function of DC which results in altered $\mathrm{T}$ cell response. Therefore, it would be of interest to assess the 
presence of these cell types in the LP during CT induced inflammation. Experiments would include flow cytometric analysis to identify populations of innate cells, including eosinophils (CD45, CD127, CCR3, Siglec F ${ }^{207,}{ }^{227}$ ), mast cells (CD34, CD117 (c-kit), FcєR1) and ILC (CD90, CD127, CD132 ${ }^{206}$ ) in the LP of naïve mice compared to mice treated with CT.

4. Genetic comparison of CD103+ TIM-4+DC to CD103+ TIM-4- DC

I was unable to detect any significant phenotypic differences between CD103+ TIM4+ DC and CD103+ TIM-4- DC from cell surface markers analysed by flow cytometry. However, I did observe functional and morphological differences between these populations. Therefore, it would be of interest to perform a more indepth approach such as transcript analysis by real time PCR that would allow the analysis of a large number of different molecules. Results from these studies could highlight potential differences that contribute to immune activation in vivo.

CD103+ TIM-4+ DC are present in the LP and MLN during the development of Th2 responses to CT. Therefore, it would be of interest to compare CD103+ TIM4+ DC to CD103+ TIM-4- DC for the expression of Th2 associated markers such as OX40L, Jagged ${ }^{86}$, IL-4R ${ }^{162}$, IL-25R, IL-33R $\alpha^{228, ~}{ }^{229}$, TSLPR and STAT6 ${ }^{162}$. In addition, cytokine transcripts could also be assessed.

5. In vitro cultures comparing T cell priming abilities of CD103+ TIM-4+ DC to CD103+ TIM4- DC

We have shown CD103+ TIM-4+ DC to have an increased capacity for taking up apoptotic cells ex vivo compared to their CD103+ TIM-4- counterparts. CD103+ TIM-4+ DC appear to maintain their capacity to migrate to the MLN and thus may 
contribute to T cell responses. Presentation of apoptotic material by mature DC has been shown previously to alter immune responses in vitro ${ }^{226}$. In addition, it is not known what effect acquisition of apoptotic material has on luminal antigen presentation. Therefore I would like to investigate whether uptake or presentation of apoptotic material alters $\mathrm{T}$ cell proliferation and differentiation. This can be achieved by assessment of T cell proliferation and differentiation following culture with FACS sorted APC subsets. 


\subsection{Final conclusions}

In this thesis I showed a novel method of identifying intestinal DC and $\mathrm{M} \varphi$ at steady-state and following administration of a mucosal adjuvant using cell surface expression of CD103 and TIM-4. In addition, I showed that CT treatment is associated with an increase in IL-4 producing $\mathrm{T}$ cells and the transient presence of a novel phenotype of DC characterised by co-expression of CD103 and TIM-4. Initial studies showed CD103+ TIM-4+ DC conferred some functional and morphological differences to steady-state CD103+ TIM-4- DC. However, further studies are required to determine the immunological significance of CD103+ TIM-4+ DC. The intestine has developed an intricate system involving signalling from many cell types to control immune responses. I propose CD103+ TIM-4+ DC do not represent a distinct DC population but rather arise due to changes in the intestinal cytokine environment. Furthermore, I propose CD103+ TIM-4+ DC may contribute to the adjuvant effects of CT. As intestinal APC populations are conserved between mice and humans, understanding of the role of CD103+ TIM-4+ DC in influencing intestinal immunity could provide insight into pathological processes that lead to diseases such as food allergy and IBD in humans. 
References 
1. Walsh KP, Mills KHG. Dendritic Cells and Other Innate Determinants of T Helper Cell Polarisation. Trends in Immunology 2013, 34(11): 521-530.

2. Kushwah R, Hu J. Complexity of Dendritic Cell Subsets and Their Function in the Host Immune System. Immunology 2011, 133(4): 409-419.

3. Sille FCM, Visser A, Boes M. T Cell Priming by Tissue-Derived Dendritic Cells: New Insights from Recent Murine Studies. Cellular immunology 2005, 237(2): 77-85.

4. Iwasaki A, Medzhitov R. Regulation of Adaptive Immunity by the Innate Immune System. Science 2010, 327(5963): 291-295.

5. Litman GW, Rast JP, Fugmann SD. The Origins of Vertebrate Adaptive Immunity. Nature Reviews Immunology 2010, 10(8): 543-553.

6. Banchereau J, Briere F, Caux C, Davoust J, Lebecque S, Liu YT, . . Palucka K. Immunobiology of Dendritic Cells. Annual Review of Immunology 2000, 18: 767-811.

7. Banchereau J, Steinman RM. Dendritic Cells and the Control of Immunity. Nature 1998, 392(6673): 245-252.

8. Azuma M, Ito D, Yagita H, Okumura K, Phillips JH, Lanier LL, Somoza C. B70 Antigen Is a 2nd Ligand for Ctla-4 and Cd28. Nature 1993, 366(6450): 76-79.

9. Saeki H, Moore AM, Brown MJ, Hwang ST. Cutting Edge: Secondary LymphoidTissue Chemokine (Slc) and Cc Chemokine Receptor 7 (Ccr7) Participate in the Emigration Pathway of Mature Dendritic Cells from the Skin to Regional Lymph Nodes. Journal of Immunology 1999, 162(5): 2472-2475. 
10. Murphy K, Travers, P., Walport, M. Immunobiology, 7 edn. Garland Science: New York, 2008.

11. Man S, Lechler RI, Batchelor JR, Sharrock CEM. Individual Variation in the Frequency of Hla Class-Ii-Specific Cytotoxic Lymphocyte-T Precursors. European Journal of Immunology 1990, 20(4): 847-854.

12. McCarthy SA, Singer A. Recognition of Mhc Class-I Allodeterminants Regulates the Generation of Mhc Class-Ii-Specific Ctl. Journal of Immunology 1986, 137(10): 30873092.

13. Guygrand D, Malassisseris M, Briottet C, Vassalli P. Cytotoxic Differentiation of Mouse Gut Thymodependent and Independent Intraepithelial Lymphocytes-T Is Induced Locally - Correlation between Functional Assays, Presence of Perforin and Granzyme Transcripts, and Cytoplasmic Granules. Journal of Experimental Medicine 1991, 173(6): 1549-1552.

14. Mosmann TR, Cherwinski H, Bond MW, Giedlin MA, Coffman RL. 2 Types of Murine Helper T-Cell Clone .1. Definition According to Profiles of Lymphokine Activities and Secreted Proteins. Journal of Immunology 1986, 136(7): 2348-2357.

15. Heufler C, Koch F, Stanzl U, Topar G, Wysocka M, Trinchieri G, . . . Schuler G. Interleukin-12 Is Produced by Dendritic Cells and Mediates $T$ Helper 1 Development as Well as Interferon-Gamma Production by T Helper 1 Cells. European Journal of Immunology 1996, 26(3): 659-668.

16. Bettelli E, Carrier YJ, Gao WD, Korn T, Strom TB, Oukka M, . . Kuchroo VK. Reciprocal Developmental Pathways for the Generation of Pathogenic Effector T(H)17 and Regulatory T Cells. Nature 2006, 441(7090): 235-238. 
17. Mangan PR, Harrington LE, O'Quinn DB, Helms WS, Bullard DC, Elson CO, ... Weaver CT. Transforming Growth Factor-Beta Induces Development of the T(H)17 Lineage. Nature 2006, 441(7090): 231-234.

18. Kaiko GE, Horvat JC, Beagley KW, Hansbro PM. Immunological Decision-Making: How Does the Immune System Decide to Mount a Helper T-Cell Response? Immunology 2008, 123(3): 326-338.

19. Mahon BP, Katrak K, Nomoto A, Macadam AJ, Minor PD, Mills KHG. PoliovirusSpecific Cd4(+) Th1 Clones with Both Cytotoxic and Helper Activity Mediate Protective Humoral Immunity against a Lethal Poliovirus Infection in Transgenic Mice Expressing the Human Poliovirus Receptor. Journal of Experimental Medicine 1995, 181(4): 1285-1292.

20. Lu Y-J, Gross J, Bogaert D, Finn A, Bagrade L, Zhang Q, . . Malley R. Interleukin17a Mediates Acquired Immunity to Pneumococcal Colonization. PLoS Pathog 2008, 4(9): e1000159.

21. Le Gros G, Ben-Sasson SZ, Seder R, Finkelman FD, Paul WE. Generation of Interleukin 4 (Il-4)-Producing Cells in Vivo and in Vitro: Il-2 and Il-4 Are Required for in Vitro Generation of Il-4-Producing Cells. J Exp Med 1990, 172(3): 921-929.

22. Seder RA, Paul WE, Davis MM, Fazekas de St Groth B. The Presence of Interleukin 4 During in Vitro Priming Determines the Lymphokine-Producing Potential of Cd4+ T Cells from T Cell Receptor Transgenic Mice. J Exp Med 1992, 176(4): 10911098.

23. McKenzie GJ, Fallon PG, Emson CL, Grencis RK, McKenzie ANJ. Simultaneous Disruption of Interleukin (Il)-4 and Il-13 Defines Individual Roles in T Helper Cell Type 2-Mediated Responses. Journal of Experimental Medicine 1999, 189(10): 15651572. 
24. Tomoki Ito Y-HW, Omar Duramad, Toshiyuki Hori. Tslp-Activated Dendritic Cells Induce an Inflammatory T Helper 2 Cell Response through Ox401. The Journal of Experimental Medicine 2005, 202(9): 1213-1223.

25. Allen JE, Maizels RM. Diversity and Dialogue in Immunity to Helminths. Nature Reviews Immunology 2011, 11(6): 375-388.

26. Workman CJ, Szymczak-Workman AL, Collison LW, Pillai MR, Vignali DAA. The Development and Function of Regulatory T Cells. Cellular and Molecular Life Sciences 2009, 66(16): 2603-2622.

27. Kushwah R, Hu J. Role of Dendritic Cells in the Induction of Regulatory T Cells. Cell and Bioscience 2011, 1: 10.

28. Hegazy AN, Peine M, Helmstetter C, Panse I, Frohlich A, Bergthaler A, ... Lohning M. Interferons Direct Th2 Cell Reprogramming to Generate a Stable Gata-3(+)TBet(+) Cell Subset with Combined Th2 and Th1 Cell Functions. Immunity 2010, 32(1): 116-128.

29. Zhu JF, Min B, Hu-Li J, Watson CJ, Grinberg A, Wang Q, . . Paul WE. Conditional Deletion of Gata3 Shows Its Essential Function in $\mathrm{T}(\mathrm{H}) 1-\mathrm{T}(\mathrm{H}) 2$ Responses. Nature Immunology 2004, 5(11): 1157-1165.

30. Murphy E, Shibuya K, Hosken N, Openshaw P, Maino V, Davis K, ... Ogarra A. Reversibility of T Helper 1 and 2 Populations Is Lost after Long-Term Stimulation. Journal of Experimental Medicine 1996, 183(3): 901-913.

31. Lee YK, Turner H, Maynard CL, Oliver JR, Chen DQ, Elson CO, Weaver CT. Late Developmental Plasticity in the T Helper 17 Lineage. Immunity 2009, 30(1): 92-107.

32. Zhu JF, Paul WE. Heterogeneity and Plasticity of T Helper Cells. Cell Research 2010, 20(1): 4-12. 
33. Xu LL, Kitani A, Fuss I, Strober W. Cutting Edge: Regulatory T Cells Induce Cd4(+)Cd25(-)Foxp3(-) T Cells or Are Self-Induced to Become Th17 Cells in the Absence of Exogenous Tgf-Beta. Journal of Immunology 2007, 178(11): 6725-6729.

34. Komatsu N, Mariotti-Ferrandiz ME, Wang Y, Malissen B, Waldmann H, Hori S. Heterogeneity of Natural Foxp3(+) T Cells: A Committed Regulatory T-Cell Lineage and an Uncommitted Minor Population Retaining Plasticity. Proceedings of the National Academy of Sciences of the United States of America 2009, 106(6): 1903-1908.

35. Soroosh P, Doherty TA. Th9 and Allergic Disease. Immunology 2009, 127(4): 450-458.

36. Basu R, O'Quinn DB, Silberger DJ, Schoeb TR, Fouser L, Ouyang WJ, ... Weaver CT. Th22 Cells Are an Important Source of Il-22 for Host Protection against Enteropathogenic Bacteria. Immunity 2012, 37(6): 1061-1075.

37. Metlay JP, Witmerpack MD, Agger R, Crowley MT, Lawless D, Steinman RM. The Distinct Leukocyte Integrins of Mouse Spleen Dendritic Cells as Identified with New Hamster Monoclonal-Antibodies. Journal of Experimental Medicine 1990, 171(5): 17531771.

38. Hume DA. Macrophages as Apc and the Dendritic Cell Myth. Journal of Immunology 2008, 181(9): 5829-5835.

39. Probst HC, Tschannen K, Odermatt B, Schwendener R, Zinkernagel RM, Van Den Broek M. Histological Analysis of Cd11c-Dtr/Gfp Mice after in Vivo Depletion of Dendritic Cells. Clinical and Experimental Immunology 2005, 141(3): 398-404.

40. van Rijt LS, Jung S, KleinJan A, Vos N, Willart M, Duez C, . . Lambrecht BN. In Vivo Depletion of Lung Cd11c(+) Dendritic Cells During Allergen Challenge Abrogates the Characteristic Features of Asthma. Journal of Experimental Medicine 2005, 201(6): 981-991. 
41. Soos TJ, Sims TN, Barisoni L, Lin K, Littman DR, Dustin ML, Nelson PJ. Cx(3)Cr1(+) Interstitial Dendritic Cells Form a Contiguous Network Throughout the Entire Kidney. Kidney International 2006, 70(3): 591-596.

42. Schulz O, Jaensson E, Persson EK, Liu X, Worbs T, Agace WW, Pabst O. Intestinal Cd103(+), but Not Cx3cr1(+), Antigen Sampling Cells Migrate in Lymph and Serve Classical Dendritic Cell Functions. Journal of Experimental Medicine 2009, 206(13): 3101-3114.

43. Keizer GD, Borst J, Visser W, Schwarting R, Devries JE, Figdor CG. Membrane Glycoprotein-P150,95 of Human Cytotoxic T-Cell Clones Is Involved in Conjugate Formation with Target-Cells. Journal of Immunology 1987, 138(10): 3130-3136.

44. Vermaelen KY, Carro-Muino I, Lambrecht BN, Pauwels RA. Specific Migratory Dendritic Cells Rapidly Transport Antigen from the Airways to the Thoracic Lymph Nodes. Journal of Experimental Medicine 2001, 193(1): 51-60.

45. Liu K, Nussenzweig MC. Origin and Development of Dendritic Cells. Immunological Reviews 2010, 234: 45-54.

46. Colonna M, Trinchieri G, Liu YJ. Plasmacytoid Dendritic Cells in Immunity. Nature Immunology 2004, 5(12): 1219-1226.

47. Zhang JQ, Raper A, Sugita N, Hingorani R, Salio M, Palmowski MJ, . . Crocker PR. Characterization of Siglec-H as a Novel Endocytic Receptor Expressed on Murine Plasmacytoid Dendritic Cell Precursors. Blood 2006, 107(9): 3600-3608.

48. Swiecki M, Colonna M. Unraveling the Functions of Plasmacytoid Dendritic Cells During Viral Infections, Autoimmunity, and Tolerance. Immunological Reviews 2010, 234: $142-162$. 
49. Hammad H, Plantinga M, Deswarte K, Pouliot P, Willart MAM, Kool M, ... Lambrecht BN. Inflammatory Dendritic Cells-Not Basophils-Are Necessary and Sufficient for Induction of Th2 Immunity to Inhaled House Dust Mite Allergen. Journal of Experimental Medicine 2010, 207(10): 2097-2111.

50. Langlet C, Tamoutounour S, Henri S, Luche H, Ardouin L, Gregoire C, ... Guilliams M. Cd64 Expression Distinguishes Monocyte-Derived and Conventional Dendritic Cells and Reveals Their Distinct Role During Intramuscular Immunization. Journal of Immunology 2012, 188(4): 1751-1760.

51. Kuhn S, Hyde EJ, Yang JP, Rich FJ, Harper JL, Kirman JR, Ronchese F. Increased Numbers of Monocyte-Derived Dendritic Cells During Successful Tumor Immunotherapy with Immune-Activating Agents. Journal of Immunology 2013, 191(4): 1984-1992.

52. Faria AMC, Weiner HL. Oral Tolerance. Immunological Reviews 2005, 206: 232-259.

53. Corthesy B. Roundtrip Ticket for Secretory Iga: Role in Mucosal Homeostasis? Journal of Immunology 2007, 178(1): 27-32.

54. Rescigno M. The Intestinal Epithelial Barrier in the Control of Homeostasis and Immunity. Trends in Immunology 2011, 32(6): 256-264.

55. Worbs T, Bode U, Yan S, Hoffmann MW, Hintzen G, Bernhardt G, . . Pabst O. Oral Tolerance Originates in the Intestinal Immune System and Relies on Antigen Carriage by Dendritic Cells. Journal of Experimental Medicine 2006, 203(3): 519-527.

56. Mazzini E, Massimiliano L, Penna G, Rescigno M. Oral Tolerance Can Be Established Via Gap Junction Transfer of Fed Antigens from Cx3cr1+ Macrophages to Cd103+ Dendritic Cells. Immunity 2014, 40(2): 248-261. 
57. Coombes JL, Siddiqui KR, Arancibia-Carcamo CV, Hall J, Sun CM, Belkaid Y, Powrie F. A Functionally Specialized Population of Mucosal Cd103+ Dcs Induces Foxp3+ Regulatory $\mathrm{T}$ Cells Via a Tgf-Beta and Retinoic Acid-Dependent Mechanism. J Exp Med 2007, 204(8): 1757-1764.

58. Sun CM, Hall JA, Blank RB, Bouladoux N, Oukka M, Mora JR, Belkaid Y. Small Intestine Lamina Propria Dendritic Cells Promote De Novo Generation of Foxp3 T Reg Cells Via Retinoic Acid. Journal of Experimental Medicine 2007, 204(8): 1775-1785.

59. Fazekas de St Groth B. Dcs and Peripheral T Cell Tolerance. Seminars in Immunology 2001, 13(5): 311-321.

60. Hadis U, Wahl B, Schulz O, Hardtke-Wolenski M, Schippers A, Wagner N, .. Pabst O. Intestinal Tolerance Requires Gut Homing and Expansion of Foxp3(+) Regulatory T Cells in the Lamina Propria. Immunity 2011, 34(2): 237-246.

61. Melamed D, Friedman A. Direct Evidence for Anergy in T-Lymphocytes Tolerized by Oral-Administration of Ovalbumin. European Journal of Immunology 1993, 23(4): 935-942.

62. Johansson MEV, Phillipson M, Petersson J, Velcich A, Holm L, Hansson GC. The Inner of the Two Muc2 Mucin-Dependent Mucus Layers in Colon Is Devoid of Bacteria. Proceedings of the National Academy of Sciences of the United States of America 2008, 105(39): 15064-15069.

63. Lai YP, Gallo RL. Amped up Immunity: How Antimicrobial Peptides Have Multiple Roles in Immune Defense. Trends in Immunology 2009, 30(3): 131-141.

64. Belkaid Y, Bouladoux N, Hand TW. Effector and Memory T Cell Responses to Commensal Bacteria. Trends in Immunology 2013, 34(6): 299-306. 
65. Geuking MB, Cahenzli J, Lawson MAE, Ng DCK, Slack E, Hapfelmeier S, . . . Macpherson AJ. Intestinal Bacterial Colonization Induces Mutualistic Regulatory $\mathrm{T}$ Cell Responses. Immunity 2011, 34(5): 794-806.

66. Rakoff-Nahoum S, Paglino J, Eslami-Varzaneh F, Edberg S, Medzhitov R. Recognition of Commensal Microflora by Toll-Like Receptors Is Required for Intestinal Homeostasis. Cell 2004, 118(2): 229-241.

67. Hooper LV, Littman DR, Macpherson AJ. Interactions between the Microbiota and the Immune System. Science 2012, 336(6086): 1268-1273.

68. Rimoldi M, Chieppa M, Larghi P, Vulcano M, Allavena P, Rescigno M. MonocyteDerived Dendritic Cells Activated by Bacteria or by Bacteria-Stimulated Epithelial Cells Are Functionally Different. Blood 2005, 106(8): 2818-2826.

69. Hoshi N, Schenten D, Nish SA, Walther Z, Gagliani N, Flavell RA, . . Medzhitov R. Myd88 Signalling in Colonic Mononuclear Phagocytes Drives Colitis in Il-10Deficient Mice. Nature Communications 2012, 3: 1120.

70. Arques JL, Hautefort I, Ivory K, Bertelli E, Regoli M, Clare S, . . . Nicoletti C. Salmonella Induces Flagellin- and Myd88-Dependent Migration of BacteriaCapturing Dendritic Cells into the Gut Lumen. Gastroenterology 2009, 137(2): 579-587.

71. Chieppa M, Rescigno M, Huang AYC, Germain RN. Dynamic Imaging of Dendritic Cell Extension into the Small Bowel Lumen in Response to Epithelial Cell Tlr Engagement. Journal of Experimental Medicine 2006, 203(13): 2841-2852.

72. Farache J, Koren I, Milo I, Gurevich I, Kim K-W, Zigmond E, . . . Shakhar G. Luminal Bacteria Recruit Cd103+ Dendritic Cells into the Intestinal Epithelium to Sample Bacterial Antigens for Presentation. Immunity 2013, 38(3): 581-595. 
73. Fujimoto K, Karuppuchamy T, Takemura N, Shimohigoshi M, Machida T, Haseda Y, . . Uematsu S. A New Subset of Cd103(+)Cd8 Alpha(+) Dendritic Cells in the Small Intestine Expresses Tlr3, Tlr7, and Tlr9 and Induces Th1 Response and Ctl Activity. Journal of Immunology 2011, 186(11): 6287-6295.

74. Sierro F, Dubois B, Coste A, Kaiserlian D, Kraehenbuhl JP, Sirard JC. Flagellin Stimulation of Intestinal Epithelial Cells Triggers Ccl20-Mediated Migration of Dendritic Cells. Proceedings of the National Academy of Sciences of the United States of America 2001, 98(24): 13722-13727.

75. Niess JH, Brand S, Gu XB, Landsman L, Jung S, McCormick BA, . . Reinecker HC. $\mathrm{Cx}(3) \mathrm{Cr} 1-$ Mediated Dendritic Cell Access to the Intestinal Lumen and Bacterial Clearance. Science 2005, 307(5707): 254-258.

76. Rescigno M, Urbano M, Valzasina B, Francolini M, Rotta G, Bonasio R, . . . Ricciardi-Castagnoli P. Dendritic Cells Express Tight Junction Proteins and Penetrate Gut Epithelial Monolayers to Sample Bacteria. Nature Immunology 2001, 2(4): 361-367.

77. Coombes JL, Powrie F. Dendritic Cells in Intestinal Immune Regulation. Nature Reviews Immunology 2008, 8(Suppl. S): 435-446.

78. Rimoldi M, Chieppa M, Salucci V, Avogadri F, Sonzogni A, Sampietro GM, . . . Rescigno M. Intestinal Immune Homeostasis Is Regulated by the Crosstalk between Epithelial Cells and Dendritic Cells. Nature Immunology 2005, 6(5): 507-514.

79. Ivison SM, Himmel ME, Mayer M, Yao Y, Kifayet A, Levings MK, Steiner TS. The Stress Signal Extracellular Atp Modulates Antiflagellin Immune Responses in Intestinal Epithelial Cells. Inflammatory Bowel Diseases 2011, 17(1): 319-333.

80. Denning TL, Wang YC, Patel SR, Williams IR, Pulendran B. Lamina Propria Macrophages and Dendritic Cells Differentially Induce Regulatory and Interleukin 17-Producing T Cell Responses. Nature Immunology 2007, 8(10): 1086-1094. 
81. Welty NE, Staley C, Ghilardi N, Sadowsky MJ, Igyarto BZ, Kaplan DH. Intestinal Lamina Propria Dendritic Cells Maintain T Cell Homeostasis but Do Not Affect Commensalism. Journal of Experimental Medicine 2013, 210(10): 2011-2024.

82. Nagler CR. Introduction to Special Issue on Food Allergy. Seminars in immunopathology 2012, 34(5): 615-616.

83. Berin MC, Sampson HA. Food Allergy: An Enigmatic Epidemic. Trends in Immunology 2013, 34(8): 390-397.

84. Kappelman MD, Rifas-Shiman SL, Kleinman K, Ollendorf D, Bousvaros A, Grand RJ, Finkelstein JA. The Prevalence and Geographic Distribution of Crohn's Disease and Ulcerative Colitis in the United States. Clinical Gastroenterology and Hepatology 2007, 5(12): 1424-1429.

85. Mullins RJ, Dear KBG, Tang MLK. Characteristics of Childhood Peanut Allergy in the Australian Capital Territory, 1995 to 2007. Journal of Allergy and Clinical Immunology 2009, 123(3): 689-693.

86. Blazquez AB, Berin MC. Gastrointestinal Dendritic Cells Promote Th2 Skewing Via Ox401. Journal of Immunology 2008, 180(7): 4441-4450.

87. Knight AK, Blazquez AB, Zhang S, Mayer L, Sampson HA, Berin MC. Cd4 T Cells Activated in the Mesenteric Lymph Node Mediate Gastrointestinal Food Allergy in Mice. American Journal of Physiology-Gastrointestinal and Liver Physiology 2007, 293(6): G1234-G1243.

88. Brandt EB, Strait RT, Hershko D, Wang Q, Muntel EE, Scribner TA, ... Rothenberg ME. Mast Cells Are Required for Experimental Oral Allergen-Induced Diarrhea. Journal of Clinical Investigation 2003, 112(11): 1666-1677. 
89. Dunkin D, Berin MC, Mayer L. Allergic Sensitization Can Be Induced Via Multiple Physiologic Routes in an Adjuvant-Dependent Manner. Journal of Allergy and Clinical Immunology 2011, 128(6): 1251-U1593.

90. Feehley T, Stefka AT, Cao S, Nagler CR. Microbial Regulation of Allergic Responses to Food. Seminars in immunopathology 2012, 34(5): 671-688.

91. Penders J, Gerhold K, Stobberingh EE, Thijs C, Zimmermann K, Lau S, Hamelmann E. Establishment of the Intestinal Microbiota and Its Role for Atopic Dermatitis in Early Childhood. Journal of Allergy and Clinical Immunology 2013, 132(3): 601-607.

92. Bisgaard H, Li N, Bonnelykke K, Chawes BLK, Skov T, Paludan-Muller G, . . . Krogfelt KA. Reduced Diversity of the Intestinal Microbiota During Infancy Is Associated with Increased Risk of Allergic Disease at School Age. Journal of Allergy and Clinical Immunology 2011, 128(3): 646-U318.

93. McLoughlin RM, Mills KHG. Influence of Gastrointestinal Commensal Bacteria on the Immune Responses That Mediate Allergy and Asthma. Journal of Allergy and Clinical Immunology 2011, 127(5): 1097-1107.

94. Hill DA, Siracusa MC, Abt MC, Kim BS, Kobuley D, Kubo M, ... Artis D. Commensal Bacteria-Derived Signals Regulate Basophil Hematopoiesis and Allergic Inflammation. Nature Medicine 2012, 18(4): 538-546.

95. Bates J, Diehl L. Dendritic Cells in Ibd Pathogenesis: An Area of Therapeutic Opportunity? Journal of Pathology 2014, 232(2): 112-120.

96. Baumgart DC, Carding SR. Gastroenterology 1 - Inflammatory Bowel Disease: Cause and Immunobiology. Lancet 2007, 369(9573): 1627-1640. 
97. Silva MA. Intestinal Dendritic Cells and Epithelial Barrier Dysfunction in Crohn's Disease. Inflammatory Bowel Diseases 2009, 15(3): 436-453.

98. Krajina T, Leithauser F, Moller P, Trobonjaca Z, Reimann J. Colonic Lamina Propria Dendritic Cells in Mice with $\mathrm{Cd} 4(+) \mathrm{T}$ Cell-Induced Colitis. European Journal of Immunology 2003, 33(4): 1073-1083.

99. Zigmond E, Varol C, Farache J, Elmaliah E, Satpathy AT, Friedlander G, . . Jung S. Ly6c $(\mathrm{Hi})$ Monocytes in the Inflamed Colon Give Rise to Proinflammatory Effector Cells and Migratory Antigen-Presenting Cells. Immunity 2012, 37(6): 1076-1090.

100. Rescigno M, Di Sabatino A. Dendritic Cells in Intestinal Homeostasis and Disease. Journal of Clinical Investigation 2009, 119(9): 2441-2450.

101. Bogunovic M, Ginhoux F, Helft J, Shang LM, Hashimoto D, Greter M, . . Merad M. Origin of the Lamina Propria Dendritic Cell Network. Immunity 2009, 31(3): 513525 .

102. Denning TL, Norris BA, Medina-Contreras O, Manicassamy S, Geem D, Madan R, . . Pulendran B. Functional Specializations of Intestinal Dendritic Cell and Macrophage Subsets That Control Th17 and Regulatory T Cell Responses Are Dependent on the T Cell/Apc Ratio, Source of Mouse Strain, and Regional Localization. Journal of Immunology 2011, 187(2): 733-747.

103. Satpathy AT, Briseno CG, Lee JS, Ng D, Manieri NA, Wumesh KC, ... Murphy KM. Notch2-Dependent Classical Dendritic Cells Orchestrate Intestinal Immunity to Attaching-and-Effacing Bacterial Pathogens. Nature Immunology 2013, 14(9): 937948.

104. Edelson BT, Wumesh KC, Juang R, Kohyama M, Benoit LA, Klekotka PA, . . . Murphy KM. Peripheral Cd103(+) Dendritic Cells Form a Unified Subset Developmentally Related to Cd8 Alpha(+) Conventional Dendritic Cells. Journal of Experimental Medicine 2010, 207(4): 823-836. 
105. Varol C, Vallon-Eberhard A, Elinav E, Aychek T, Shapira Y, Luche H, . . Jung S. Intestinal Lamina Propria Dendritic Cell Subsets Have Different Origin and Functions. Immunity 2009, 31(3): 502-512.

106. Cerovic V, Houston SA, Scott CL, Aumeunier A, Yrlid U, Mowat AM, Milling SWF. Intestinal Cd103- Dendritic Cells Migrate in Lymph and Prime Effector T Cells. Mucosal Immunol 2012, 6(1): 104-113.

107. Lewis KL, Caton ML, Bogunovic M, Greter M, Grajkowska LT, Ng D, . . Reizis B. Notch2 Receptor Signaling Controls Functional Differentiation of Dendritic Cells in the Spleen and Intestine. Immunity 2011, 35(5): 780-791.

108. Persson EK, Uronen-Hansson H, Semmrich M, Rivollier A, Hagerbrand K, Marsal J, ... Agace WW. Irf4 Transcription-Factor-Dependent Cd103(+)Cd11b(+) Dendritic Cells Drive Mucosal T Helper 17 Cell Differentiation. Immunity 2013, 38(5): 958-969.

109. Jaensson E, Uronen-Hansson H, Pabst O, Eksteen B, Tian J, Coombes JL, . . . Agace WW. Small Intestinal Cd103(+) Dendritic Cells Display Unique Functional Properties That Are Conserved between Mice and Humans. Journal of Experimental Medicine 2008, 205(9): 2139-2149.

110. McDole JR, Wheeler LW, McDonald KG, Wang B, Konjufca V, Knoop KA, . . . Miller MJ. Goblet Cells Deliver Luminal Antigen to Cd103(+) Dendritic Cells in the Small Intestine. Nature 2012, 483(7389): 345-U141.

111. Jang MH, Sougawa N, Tanaka T, Hirata T, Hiroi T, Tohya K, . . Miyasaka M. Ccr7 Is Critically Important in Intestinal Lamina Propria for Migration of Dendritic Cells to Mesenteric Lymph Nodes. Journal of Immunology 2006, 176(2): 803-810.

112. Varol C, Landsman L, Fogg DK, Greenshtein L, Gildor B, Margalit R, . . Jung S. Monocytes Give Rise to Mucosal, but Not Splenic, Conventional Dendritic Cells. Journal of Experimental Medicine 2007, 204(1): 171-180. 
113. Tamoutounour S, Henri S, Lelouard H, de Bovis B, de Haar C, van der Woude CJ,... . Guilliams M. Cd64 Distinguishes Macrophages from Dendritic Cells in the Gut and Reveals the Th1-Inducing Role of Mesenteric Lymph Node Macrophages During Colitis. European Journal of Immunology 2012, 42(12): 3150-3166.

114. Rivollier A, He JP, Kole A, Valatas V, Kelsall BL. Inflammation Switches the Differentiation Program of Ly6c(Hi) Monocytes from Antiinflammatory Macrophages to Inflammatory Dendritic Cells in the Colon. Journal of Experimental Medicine 2012, 209(1): 139-155.

115. Satpathy AT, Wumesh KC, Albring JC, Edelson BT, Kretzer NM, Bhattacharya D, . .. Murphy KM. Zbtb46 Expression Distinguishes Classical Dendritic Cells and Their Committed Progenitors from Other Immune Lineages. Journal of Experimental Medicine 2012, 209(6): 1135-1152.

116. Diehl GE, Longman RS, Zhang JX, Breart B, Galan C, Cuesta A, ... Littman DR. Microbiota Restricts Trafficking of Bacteria to Mesenteric Lymph Nodes by Cx(3)Cr1(Hi) Cells. Nature 2013, 494(7435): 116-120.

117. Platt AM, Bain CC, Bordon Y, Sester DP, Mowat AM. An Independent Subset of Tlr Expressing Ccr2-Dependent Macrophages Promotes Colonic Inflammation. Journal of Immunology 2010, 184(12): 6843-6854.

118. van der Poel CE, Spaapen RM, de Winkel JGJ, Leusen JHW. Functional Characteristics of the High Affinity Igg Receptor, Fc Gamma Ri. Journal of Immunology 2011, 186(5): 2699-2704.

119. Austyn JM, Gordon S. F4/80, a Monoclonal Antibody Directed Specifically against the Mouse Macrophage. European Journal of Immunology 1981, 11(10): 805-815.

120. McKnight AJ, Gordon S. The Egf-Tm7 Family: Unusual Structures at the Leukocyte Surface. Journal of Lenkocyte Biology 1998, 63(3): 271-280. 
121. Lin HH, Faunce DE, Stacey M, Terajewicz A, Nakamura T, Zhang-Hoover J, . . . Stein-Streilein J. The Macrophage F4/80 Receptor Is Required for the Induction of Antigen-Specific Efferent Regulatory $\mathrm{T}$ Cells in Peripheral Tolerance. Journal of Experimental Medicine 2005, 201(10): 1615-1625.

122. Dumont FJ. Stimulation of Murine T Cells Via the Ly-6c Antigen: Lack of Proliferative Response in Aberrant T Cells from Lpr/Lpr and Gld/Gld Mice Despite High Ly-6c Antigen Expression. J Immunol 1987, 138(12): 4106-4113.

123. Johnson R, Lancki DW, Fitch FW. Accessory Molecules Involved in AntigenMediated Cytolysis and Lymphokine Production by Cytotoxic T-Lymphocyte Subsets .1. Identification of Functions for the T-Cell Surface Molecules Ly-6c and Thy-1. Journal of Immunology 1993, 151(6): 2986-2999.

124. Hanninen A, Maksimow M, Alam C, Morgan DJ, Jalkanen S. Ly6c Supports Preferential Homing of Central Memory Cd8(+) T Cells into Lymph Nodes. European Journal of Immunology 2011, 41(3): 634-644.

125. Elias KM, Laurence A, Davidson TS, Stephens G, Kanno Y, Shevach EM, O'Shea JJ. Retinoic Acid Inhibits Th17 Polarization and Enhances Foxp3 Expression through a Stat-3/Stat-5 Independent Signaling Pathway. Blood 2008, 111(3): 10131020 .

126. Xiao S, Jin H, Korn T, Liu SM, Oukka M, Lim B, Kuchroo VK. Retinoic Acid Increases Foxp3(+) Regulatory T Cells and Inhibits Development of Th17 Cells by Enhancing Tgf-Beta-Driven Smad3 Signaling and Inhibiting Il-6 and Il-23 Receptor Expression. Journal of Immunology 2008, 181(4): 2277-2284.

127. Spadoni I, Iliev ID, Rossi G, Rescigno M. Dendritic Cells Produce Tslp That Limits the Differentiation of Th17 Cells, Fosters Treg Development, and Protects against Colitis. Mucosal Immunology 2012, 5(2): 184-193. 
128. Mora JR, Iwata M, Eksteen B, Song S-Y, Junt T, Senman B, . . von Andrian UH. Generation of Gut-Homing Iga-Secreting B Cells by Intestinal Dendritic Cells. Science 2006, 314(5802): 1157-1160.

129. Mora JR, von Andrian UH. Role of Retinoic Acid in the Imprinting of Gut-Homing Iga-Secreting Cells. Seminars in Immunology 2009, 21(1): 28-35.

130. Murai M, Turovskaya O, Kim G, Madan R, Karp CL, Cheroutre H, Kronenberg M. Interleukin 10 Acts on Regulatory $\mathrm{T}$ Cells to Maintain Expression of the Transcription Factor Foxp3 and Suppressive Function in Mice with Colitis. Nature Immunology 2009, 10(11): 1178-U1161.

131. Mizutani N, Sakurai T, Shibata T, Uchida K, Fujita J, Kawashima R, . . . Dohi T. Dose-Dependent Differential Regulation of Cytokine Secretion from Macrophages by Fractalkine. Journal of Immunology 2007, 179(11): 7478-7487.

132. Auray G, Lacroix-Lamande S, Mancassola R, Dimier-Poisson I, Laurent F. Involvement of Intestinal Epithelial Cells in Dendritic Cell Recruitment During CParvum Infection. Microbes and Infection 2007, 9(5): 574-582.

133. Uematsu S, Fujimoto K, Jang MH, Yang BG, Jung YJ, Nishiyama M, . . Akira S. Regulation of Humoral and Cellular Gut Immunity by Lamina Propria Dendritic Cells Expressing Toll-Like Receptor 5. Nature Immunology 2008, 9(7): 769-776.

134. Weber B, Saurer L, Schenk M, Dickgreber N, Mueller C. Cx3cr1 Defines Functionally Distinct Intestinal Mononuclear Phagocyte Subsets Which Maintain Their Respective Functions During Homeostatic and Inflammatory Conditions. European Journal of Immunology 2011, 41(3): 773-779.

135. Siddiqui KRR, Laffont S, Powrie F. E-Cadherin Marks a Subset of Inflammatory Dendritic Cells That Promote T Cell-Mediated Colitis. Immunity 2010, 32(4): 557-567. 
136. Schreiber HA, Loschko J, Karssemeijer RA, Escolano A, Meredith MM, Mucida D, . .. Nussenzweig MC. Intestinal Monocytes and Macrophages Are Required for T Cell Polarization in Response to Citrobacter Rodentium. Journal of Experimental Medicine 2013, 210(10): 2025-2039.

137. Mucida D, Park Y, Kim G, Turovskaya O, Scott I, Kronenberg M, Cheroutre H. Reciprocal T(H)17 and Regulatory T Cell Differentiation Mediated by Retinoic Acid. Science 2007, 317(5835): 256-260.

138. Zheng Y, Valdez PA, Danilenko DM, Hu Y, Sa SM, Gong Q, ... Ouyang W. Interleukin-22 Mediates Early Host Defense against Attaching and Effacing Bacterial Pathogens. Nature Medicine 2008, 14(3): 282-289.

139. Yamane H, Paul WE. Early Signaling Events That Underlie Fate Decisions of Naive Cd4(+) T Cells toward Distinct T-Helper Cell Subsets. Immunological Reviews 2013, 252: $12-23$.

140. Deckers J, Madeira FB, Hammad H. Innate Immune Cells in Asthma. Trends in Immunology 2013, 34(11): 540-547.

141. Paul WE. What Determines Th2 Differentiation, in Vitro and in Vivo? Immunology and Cell Biology 2010, 88(3): 236-239.

142. Paul WE, Zhu JF. How Are $\mathrm{T}(\mathrm{H}) 2$-Type Immune Responses Initiated and Amplified? Nature Reviews Immunology 2010, 10(4): 225-235.

143. Smith KA, Hochweller K, Haemmerling GJ, Boon L, MacDonald AS, Maizels RM. Chronic Helminth Infection Promotes Immune Regulation in Vivo through Dominance of Cd11c(Lo)Cd103(-) Dendritic Cells. Journal of Immunology 2011, 186(12): 7098-7109. 
144. Feng BS, Chen X, He SH, Zheng PY, Foster J, Xing Z, ... Yang PC. Disruption of T-Cell Immunoglobulin and Mucin Domain Molecule (Tim)-1/Tim4 Interaction as a Therapeutic Strategy in a Dendritic Cell-Induced Peanut Allergy Model. Journal of Allergy and Clinical Immunology 2008, 122(1): 55-61.

145. Albacker LA, Yu S, Bedoret D, Lee WL, Umetsu SE, Monahan S, . . DeKruyff RH. Tim-4, Expressed by Medullary Macrophages, Regulates Respiratory Tolerance by Mediating Phagocytosis of Antigen-Specific T Cells. Mucosal Immunology 2013, 6(3): 580-590.

146. Wong K, Valdez PA, Tan C, Yeh S, Hongo JA, Ouyang W. Phosphatidylserine Receptor Tim-4 Is Essential for the Maintenance of the Homeostatic State of Resident Peritoneal Macrophages. Proceedings of the National Academy of Sciences of the United States of America 2010, 107(19): 8712-8717.

147. Meyers JH, Chakravarti S, Schlesinger D, Illes Z, Waldner H, Umetsu SE, . . . Kuchroo VK. Tim-4 Is the Ligand for Tim-1, and the Tim-1-Tim-4 Interaction Regulates T Cell Proliferation. Nature Immunology 2005, 6(5): 455-464.

148. Rodriguez-Manzanet R, Meyers JH, Balasubramanian S, Slavik J, Kassam N, Dardalhon V, . . . Kuchroo VK. Tim-4 Expressed on Apcs Induces T Cell Expansion and Survival. Journal of Immunology 2008, 180(7): 4706-4713.

149. Yang P-C, Xing Z, Berin CM, Soderholm JD, Feng B-S, Wu L, Yeh C. Tim-4 Expressed by Mucosal Dendritic Cells Plays a Critical Role in Food Antigen-Specific Th2 Differentiation and Intestinal Allergy. Gastroenterology 2007, 133(5): 1522-1533.

150. Braun MC, He JP, Wu CY, Kelsall BL. Cholera Toxin Suppresses Interleukin (Il)-12 Production and Il-12 Receptor Beta 1 and Beta 2 Chain Expression. Journal of Experimental Medicine 1999, 189(3): 541-552.

151. Eriksson K, Fredriksson M, Nordstrom I, Holmgren J. Cholera Toxin and Its B Subunit Promote Dendritic Cell Vaccination with Different Influences on Th1 and Th2 Development. Infection and Immunity 2003, 71(4): 1740-1747. 
152. Gagliardi MC, Sallusto F, Marinaro M, Vendetti S, Riccomi A, De Magistris MT. Effects of the Adjuvant Cholera Toxin on Dendritic Cells: Stimulatory and Inhibitory Signals That Result in the Amplification of Immune Responses. IJMM International Journal of Medical Microbiology 2002, 291(6-7): 571-575.

153. la Sala A, He JP, Laricchia-Robbio L, Gorini S, Iwasaki A, Braun M, . . Kelsall B. Cholera Toxin Inhibits Il-12 Production and Cd8 Alpha(+) Dendritic Cell Differentiation by Camp-Mediated Inhibition of Irf8 Function. Journal of Experimental Medicine 2009, 206(6): 1227-1235.

154. Gagliardi MC, Sallusto F, Marinaro M, Langenkamp A, Lanzavecchia A, De Magistris MT. Cholera Toxin Induces Maturation of Human Dendritic Cells and Licences Them for Th2 Priming. European Journal of Immunology 2000, 30(8): 23942403.

155. Xuamano JC, Kiyono H, Jackson RJ, Staats HF, Fujihashi K, Burrows PD, . . . McGhee JR. Helper T-Cell Subsets for Immunoglobulin-a Responses - Oral Immunization with Tetanus Toxoid and Cholera-Toxin as Adjuvant Selectively Induces Th2 Cells in Mucosa-Associated Tissues. Journal of Experimental Medicine 1993, 178(4): 1309-1320.

156. Marinaro M, Staats HF, Hiroi T, Jackson RJ, Coste M, Boyaka PN, ... McGhee JR. Mucosal Adjuvant Effect of Cholera-Toxin in Mice Results from Induction of THelper-2 (Th2) Cells and Il-4. Journal of Immunology 1995, 155(10): 4621-4629.

157. Kim K-J, Kim H-A, Seo KH, Lee H-K, Kang BY, Im S-Y. Cholera Toxin Breakdowns Oral Tolerance Via Activation of Canonical Nf-Kappab. Cellular immunology 2013, 285(1-2): 92-99.

158. Anjuere F, Luci C, Lebens M, Rousseau D, Hervouet C, Milon G, ... Czerkinsky C. In Vivo Adjuvant-Induced Mobilization and Maturation of Gut Dendritic Cells after Oral Administration of Cholera Toxin. Journal of Immunology 2004, 173(8): 5103-5111. 
159. Apte SH, Redmond AM, Groves PL, Schussek S, Pattinson DJ, Doolan DL. Subcutaneous Cholera Toxin Exposure Induces Potent $\mathrm{Cd}_{103}{ }^{+}$Dermal Dendritic Cell Activation and Migration. European Journal of Immunology 2013, 43(10): 2707-2717.

160. Gustafsson T, Hua Y-J, Dahlgren MW, Livingston M, Johansson-Lindbom B, Yrlid U. Direct Interaction between Cholera Toxin and Dendritic Cells Is Required for Oral Adjuvant Activity. European Journal of Immunology 2013, 43(7): 1779-1788.

161. Kawamura YI, Kawashima R, Shirai Y, Kato R, Hamabata T, Yamamoto M, . . . Dohi T. Cholera Toxin Activates Dendritic Cells through Dependence on Gm1Ganglioside Which Is Mediated by Nf-Kappa B Translocation. European Journal of Immunology 2003, 33(11): 3205-3212.

162. Berin MC, Shreffler WG. T(H)2 Adjuvants: Implications for Food Allergy. The Journal of allergy and clinical immunology 2008, 121(6): 1311-1320; quiz 1321-1312.

163. Roediger B, Kyle R, Yip KH, Sumaria N, Guy TV, Kim BS, ... Weninger W. Cutaneous Immunosurveillance and Regulation of Inflammation by Group 2 Innate Lymphoid Cells. Nature Immunology 2013, 14(6): 564-573.

164. Hoshino K, Takeuchi O, Kawai T, Sanjo H, Ogawa T, Takeda Y, . . Akira S. Cutting Edge: Toll-Like Receptor 4 (Tlr4)-Deficient Mice Are Hyporesponsive to Lipopolysaccharide: Evidence for Tlr4 as the Lps Gene Product. Journal of Immunology 1999, 162(7): 3749-3752.

165. Barnden MJ, Allison J, Heath WR, Carbone FR. Defective Tcr Expression in Transgenic Mice Constructed Using Cdna-Based Alpha- and Beta-Chain Genes under the Control of Heterologous Regulatory Elements. Immunology and Cell Biology 1998, 76(1): 34-40.

166. Vallon-Eberhard A, Landsman L, Yogev N, Verrier B, Jung S. Transepithelial Pathogen Uptake into the Small Intestinal Lamina Propria. Journal of Immunology 2006, 176(4): 2465-2469. 
167. Annacker O, Coombes JL, Malmstrom V, Uhlig HH, Bourne T, Johansson-Lindbom B , . . Powrie F. Essential Role for Cd103 in the T Cell-Mediated Regulation of Experimental Colitis. Journal of Experimental Medicine 2005, 202(8): 1051-1061.

168. Johansson-Lindbom B, Svensson M, Pabst O, Palmqvist C, Marquez G, Forster R, Agace WW. Functional Specialization of Gut Cd103(+) Dendritic Cells in the Regulation of Tissue-Selective T Cell Homing. Journal of Experimental Medicine 2005, 202(8): 1063-1073.

169. Henri S, Guilliams M, Poulin LF, Tamoutounour S, Ardouin L, Dalod M, Malissen B. Disentangling the Complexity of the Skin Dendritic Cell Network. Immunology and Cell Biology 2010, 88(4): 366-375.

170. Desch AN, Henson PM, Jakubzick CV. Pulmonary Dendritic Cell Development and Antigen Acquisition. Immunologic Research 2013, 55(1-3): 178-186.

171. Hey YY, O'Neill HC. Murine Spleen Contains a Diversity of Myeloid and Dendritic Cells Distinct in Antigen Presenting Function. Journal of Cellular and Molecular Medicine 2012, 16(11): 2611-2619.

172. Kumamoto Y, Linehan M, Weinstein JS, Laidlaw BJ, Craft JE, Iwasaki A. Cd301b Dermal Dendritic Cells Drive T Helper 2 Cell-Mediated Immunity. Immunity 2013, 39(4): 733-743.

173. Treuting PM, Valasek MA, Dintzis SM. Upper Gastrointestinal Tract. In: Treuting PM, Dintzis SM (eds). Comparative Anatomy and Histology, 1 edn. Academic Press: San Diego, 2012, pp 155-175.

174. Kinnebrew MA, Buffie CG, Diehl GE, Zenewicz LA, Leiner I, Hohl TM, .. . Pamer EG. Interleukin 23 Production by Intestinal Cd103(+)Cd11b(+) Dendritic Cells in Response to Bacterial Flagellin Enhances Mucosal Innate Immune Defense. Immunity 2012, 36(2): 276-287. 
175. Laffont S, Siddiqui KRR, Powrie F. Intestinal Inflammation Abrogates the Tolerogenic Properties of Mln Cd103(+) Dendritic Cells. European Journal of Immunology 2010, 40(7): 1877-1883.

176. Berin MC, Mayer L. Immunophysiology of Experimental Food Allergy. Mucosal Immunology 2009, 2(1): 24-32.

177. Guichard A, Cruz-Moreno B, Aguilar B, van Sorge NM, Kuang J, Kurkciyan AA, ... Bier E. Cholera Toxin Disrupts Barrier Function by Inhibiting Exocyst-Mediated Trafficking of Host Proteins to Intestinal Cell Junctions. Cell host \& microbe 2013, 14(3): 294-305.

178. Nell S, Suerbaum S, Josenhans C. The Impact of the Microbiota on the Pathogenesis of Ibd: Lessons from Mouse Infection Models. Nature Reviews Microbiology 2010, 8(8): 564-577.

179. Cooper HS, Murthy SNS, Shah RS, Sedergran DJ. Clinicopathological Study of Dextran Sulfate Sodium Experimental Murine Colitis. Laboratory Investigation 1993, 69(2): 238-249.

180. Mann ER, Landy JD, Bernardo D, Peake STC, Hart AL, Al-Hassi HO, Knight SC. Intestinal Dendritic Cells: Their Role in Intestinal Inflammation, Manipulation by the Gut Microbiota and Differences between Mice and Men. Immunology Letters 2013, 150(1-2): 30-40.

181. Macpherson AJ, McCoy KD. Stratification and Compartmentalisation of Immunoglobulin Responses to Commensal Intestinal Microbes. Seminars in Immunology 2013, 25(5): 358-363.

182. Martin M, Metzger DJ, Michalek SM, Connell TD, Russell MW. Distinct Cytokine Regulation by Cholera Toxin and Type Ii Heat-Labile Toxins Involves Differential 
Regulation of Cd40 Ligand on Cd4(+) T Cells. Infection and Immunity 2001, 69(7): 4486-4492.

183. Bromander A, Holmgren J, Lycke N. Cholera-Toxin Stimulates Il-1 Production and Enhances Antigen Presentation by Macrophages Invitro. Journal of Immunology 1991, 146(9): 2908-2914.

184. Snider DP, Marshall JS, Perdue MH, Liang H. Production of Ige Antibody and Allergic Sensitization of Intestinal and Peripheral-Tissues after Oral Immunization with Protein Ag and Cholera-Toxin. Journal of Immunology 1994, 153(2): 647-657.

185. Adel-Patient K, Bernard H, Ah-Leung S, Creminon C, Wal JM. Peanut- and Cow's Milk-Specific Ige, Th2 Cells and Local Anaphylactic Reaction Are Induced in Balb/C Mice Orally Sensitized with Cholera Toxin. Allergy 2005, 60(5): 658-664.

186. Gagliardi MC, De Magistris MT. Maturation of Human Dendritic Cells Induced by the Adjuvant Cholera Toxin: Role of Camp on Chemokine Receptor Expression. Vaccine 2003, 21(9-10): 856-861.

187. Kobayashi N, Karisola P, Pena-Cruz V, Dorfman DM, Jinushi M, Umetsu SE, ... Freeman GJ. Tim-1 and Tim-4 Glycoproteins Bind Phosphatidylserine and Mediate Uptake of Apoptotic Cells. Immunity 2007, 27(6): 927-940.

188. Miyanishi M, Tada K, Koike M, Uchiyama Y, Kitamura T, Nagata S. Identification of Tim4 as a Phosphatidylserine Receptor. Nature 2007, 450(7168): 435-439.

189. Ganeshan K, Neilsen CV, Hadsaitong A, Schleimer RP, Luo X, Bryce PJ. Impairing Oral Tolerance Promotes Allergy and Anaphylaxis: A New Murine Food Allergy Model. Journal of Allergy and Clinical Immunology 2009, 123(1): 231-238.

190. Croxford AL, Buch T. Cytokine Reporter Mice in Immunological Research: Perspectives and Lessons Learned. Immunology 2011, 132(1): 1-8. 
191. Persson EK, Scott CL, Mowat AM, Agace WW. Dendritic Cell Subsets in the Intestinal Lamina Propria: Ontogeny and Function. European Journal of Immunology 2013, 43(12): 3098-3107.

192. Farache J, Zigmond E, Shakhar G, Jung S. Contributions of Dendritic Cells and Macrophages to Intestinal Homeostasis and Immune Defense. Immunology and Cell Biology 2013, 91(3): 232-239.

193. Watchmaker PB, Lahl K, Lee M, Baumjohann D, Mortont J, Kim SJ, . . Butcher EC. Comparative Transcriptional and Functional Profiling Defines Conserved Programs of Intestinal Dc Differentiation in Humans and Mice. Nature Immunology 2014, 15(1): 98-108.

194. Poltorak A, He XL, Smirnova I, Liu MY, Van Huffel C, Du X, . . . Beutler B. Defective Lps Signaling in C3h/Hej and C57bl/10sccr Mice: Mutations in Tlr4 Gene. Science 1998, 282(5396): 2085-2088.

195. Morafo V, Srivastava K, Huang CK, Kleiner G, Lee SY, Sampson HA, Li XM. Genetic Susceptibility to Food Allergy Is Linked to Differential T(H)2-T(H)1 Responses in $\mathrm{C} 3 \mathrm{~h} / \mathrm{Hej}$ and Balb/C Mice. Journal of Allergy and Clinical Immunology 2003, 111(5): 1122-1128.

196. Schouten B, van Esch B, Hofman GA, de Kivit S, Boon L, Knippels LMJ, ... Willemsen LEM. A Potential Role for Cd25(+) Regulatory T-Cells in the Protection against Casein Allergy by Dietary Non-Digestible Carbohydrates. British Journal of Nutrition 2012, 107(1): 96-105.

197. Holmgren J, Lonnroth I, Svennerh.L. Tissue Receptor for Cholera Exotoxin Postulated Structure from Studies with Gm1 Ganglioside and Related Glycolipids. Infection and Immunity 1973, 8(2): 208-214. 
198. Iliev ID, Mileti E, Matteoli G, Chieppa M, Rescigno M. Intestinal Epithelial Cells Promote Colitis-Protective Regulatory T-Cell Differentiation through Dendritic Cell Conditioning. Mucosal Immunology 2009, 2(4): 340-350.

199. Lundin A, Bok CM, Aronsson L, Bjorkholm B, Gustafsson JA, Pott S, . . . Pettersson S. Gut Flora, Toll-Like Receptors and Nuclear Receptors: A Tripartite Communication That Tunes Innate Immunity in Large Intestine. Cellular microbiology 2008, 10(5): 1093-1103.

200. Bandyopadhaya A, Das D, Chaudhuri K. Involvement of Intracellular Signaling Cascades in Inflammatory Responses in Human Intestinal Epithelial Cells Following Vibrio Cholerae Infection. Molecular Immunology 2009, 46(6): 1129-1139.

201. McGee DW, Elson CO, McGhee JR. Enhancing Effect of Cholera-Toxin on Interleukin-6 Secretion by Iec-6 Intestinal Epithelial-Cells - Mode of Action and Augmenting Effect of Inflammatory Cytokines. Infection and Immunity 1993, 61(11): 4637-4644.

202. Chatterjee D, Chaudhuri K. Vibrio Cholerae O395 Outer Membrane Vesicles Modulate Intestinal Epithelial Cells in a Nod1 Protein-Dependent Manner and Induce Dendritic Cell-Mediated Th2/Th17 Cell Responses. Journal of Biological Chemistry 2013, 288(6): 4299-4309.

203. Bandyopadhaya A, Sarkar M, Chaudhuri K. Transcriptional Upregulation of Inflammatory Cytokines in Human Intestinal Epithelial Cells Following Vibrio Cholerae Infection. Febs Journal 2007, 274(17): 4631-4642.

204. Svensson-Frej M. Immunobiology of Intestinal Eosinophils - a Dogma in the Changing? Journal of Innate Immunity 2011, 3(6): 565-576.

205. Kurashima Y, Goto Y, Kiyono H. Mucosal Innate Immune Cells Regulate Both Gut Homeostasis and Intestinal Inflammation. European Journal of Immunology 2013, 43(12): 3108-3115. 
206. Sonnenberg GF, Fouser LA, Artis D. Border Patrol: Regulation of Immunity, Inflammation and Tissue Homeostasis at Barrier Surfaces by Il-22. Nature Immunology 2011, 12(5): 383-390.

207. Carlens J, Wahl B, Ballmaier M, Bulfone-Paus S, Forster R, Pabst O. Common Gamma-Chain-Dependent Signals Confer Selective Survival of Eosinophils in the Murine Small Intestine. Journal of Immunology 2009, 183(9): 5600-5607.

208. Hogan SP, Mishra A, Brandt EB, Royalty MP, Pope SM, Zimmermann N, . . . Rothenberg ME. A Pathological Function for Eotaxin and Eosinophils in Eosinophilic Gastrointestinal Inflammation. Nature Immunology 2001, 2(4): 353-360.

209. Forbes E, Murase T, Yang M, Matthaei KI, Lee JJ, Lee NA, . . . Hogan SP. Immunopathogenesis of Experimental Ulcerative Colitis Is Mediated by Eosinophil Peroxidase. Journal of Immunology 2004, 172(9): 5664-5675.

210. Yang D, Chen Q, Su SB, Zhang P, Kurosaka K, Caspi RR, ... Oppenheim JJ. Eosinophil-Derived Neurotoxin Acts as an Alarmin to Activate the Tlr2-Myd88 Signal Pathway in Dendritic Cells and Enhances Th2 Immune Responses. Journal of Experimental Medicine 2008, 205(1): 79-90.

211. Kurashima Y, Amiya T, Nochi T, Fujisawa K, Haraguchi T, Iba H, . . Kiyono H. Extracellular Atp Mediates Mast Cell-Dependent Intestinal Inflammation through P2x7 Purinoceptors. Nature Communications 2012, 3: 12.

212. Mortha A, Chudnovskiy A, Hashimoto D, Bogunovic M, Spencer SP, Belkaid Y, Merad M. Microbiota-Dependent Crosstalk between Macrophages and Ilc3 Promotes Intestinal Homeostasis. Science 2014, 343(1678).

213. Stange J, Hepworth MR, Rausch S, Zajic L, Kuhl AA, Uyttenhove C, ... Lucius R. Il-22 Mediates Host Defense against an Intestinal Intracellular Parasite in the 
Absence of Ifn-Gamma at the Cost of Th17-Driven Immunopathology. Journal of Immunology 2012, 188(5): 2410-2418.

214. Aujla SJ, Chan YR, Zheng MQ, Fei MJ, Askew DJ, Pociask DA, . . Kolls JK. Il-22 Mediates Mucosal Host Defense against Gram-Negative Bacterial Pneumonia. Nature Medicine 2008, 14(3): 275-281.

215. Boniface K, Bernard FX, Garcia M, Gurney AL, Lecron JC, Morel F. Il-22 Inhibits Epidermal Differentiation and Induces Proinflammatory Gene Expression and Migration of Human Keratinocytes. Journal of Immunology 2005, 174(6): 3695-3702.

216. Zhan YF, Vega-Ramos J, Carrington EM, Villadangos JA, Lew AM, Xu YK. The Inflammatory Cytokine, Gm-Csf, Alters the Developmental Outcome of Murine Dendritic Cells. European Journal of Immunology 2012, 42(11): 2889-2900.

217. Li BZ, Ye QL, Xu WD, Li JH, Ye DQ, Xu YK. Gm-Csf Alters Dendritic Cells in Autoimmune Diseases. Autoimmunity 2013, 46(7): 409-418.

218. Egea L, Hirata Y, Kagnoff MF. Gm-Csf: A Role in Immune and Inflammatory Reactions in the Intestine. Expert Review of Gastroenterology \& Hepatology 2010, 4(6): 723-731.

219. Gloudemans AK, Plantinga M, Guilliams M, Willart MA, Ozir-Fazalalikhan A, van der Ham A, . . Smits HH. The Mucosal Adjuvant Cholera Toxin B Instructs NonMucosal Dendritic Cells to Promote Iga Production Via Retinoic Acid and Tgf-Beta. Plos One 2013, 8(3): 10.

220. Bagley KC, Abdelwahab SF, Tuskan RG, Fouts TR, Lewis GK. Cholera Toxin and Heat-Labile Enterotoxin Activate Human Monocyte-Derived Dendritic Cells and Dominantly Inhibit Ctokine Production through a Cyclic Amp-Dependent Pathway. Infection and Immunity 2002, 70(10): 5533-5539. 
221. Hall JA, Cannons JL, Grainger JR, Dos Santos LM, Hand TW, Naik S, . . Belkaid Y. Essential Role for Retinoic Acid in the Promotion of Cd4(+) T Cell Effector Responses Via Retinoic Acid Receptor Alpha. Immunity 2011, 34(3): 435-447.

222. Hammerschmidt SI, Friedrichsen M, Boelter J, Lyszkiewicz M, Kremmer E, Pabst O, Foerster R. Retinoic Acid Induces Homing of Protective T and B Cells to the Gut after Subcutaneous Immunization in Mice. Journal of Clinical Investigation 2011, 121(8): 3051-3061.

223. Iwata M, Hirakiyama A, Eshima Y, Kagechika H, Kato C, Song SY. Retinoic Acid Imprints Gut-Homing Specificity on T Cells. Immunity 2004, 21(4): 527-538.

224. Huynh MLN, Fadok VA, Henson PM. Phosphatidylserine-Dependent Ingestion of Apoptotic Cells Promotes Tgf-Beta 1 Secretion and the Resolution of Inflammation. Journal of Clinical Investigation 2002, 109(1): 41-50.

225. Sauter B, Albert ML, Francisco L, Larsson M, Somersan S, Bhardwaj N. Consequences of Cell Death: Exposure to Necrotic Tumor Cells, but Not Primary Tissue Cells or Apoptotic Cells, Induces the Maturation of Immunostimulatory Dendritic Cells. Journal of Experimental Medicine 2000, 191(3): 423-433.

226. Johansson U, Walther-Jallow L, Hofmann A, Spetz AL. Dendritic Cells Are Able to Produce Il-12p70 after Uptake of Apoptotic Cells. Immunobiology 2011, 216(1-2): 251255.

227. Stevens WW, Kim TS, Pujanauski LM, Hao X, Braciale TJ. Detection and Quantitation of Eosinophils in the Murine Respiratory Tract by Flow Cytometry. J Immunol Methods 2007, 327(1-2): 63-74.

228. Nakanishi W, Yamaguchi S, Matsuda A, Suzukawa M, Shibui A, Nambu A, ... Nakae S. Il-33, but Not Il-25, Is Crucial for the Development of House Dust Mite Antigen-Induced Allergic Rhinitis. Plos One 2013, 8(10). 
229. Salimi M, Barlow JL, Saunders SP, Xue LZ, Gutowska-Owsiak D, Wang XW, . . . Ogg GS. A Role for Il-25 and Il-33-Driven Type-2 Innate Lymphoid Cells in Atopic Dermatitis. Journal of Experimental Medicine 2013, 210(13): 2939-2950. 
Appendices 

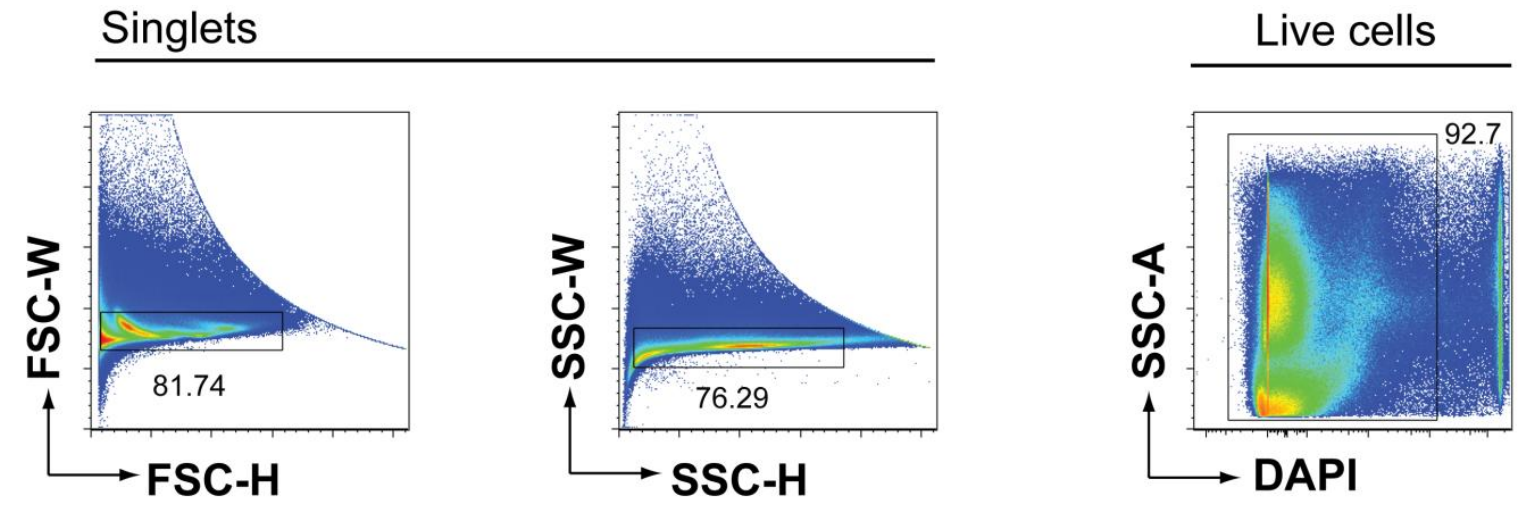

Immune cells

Dendritic cells
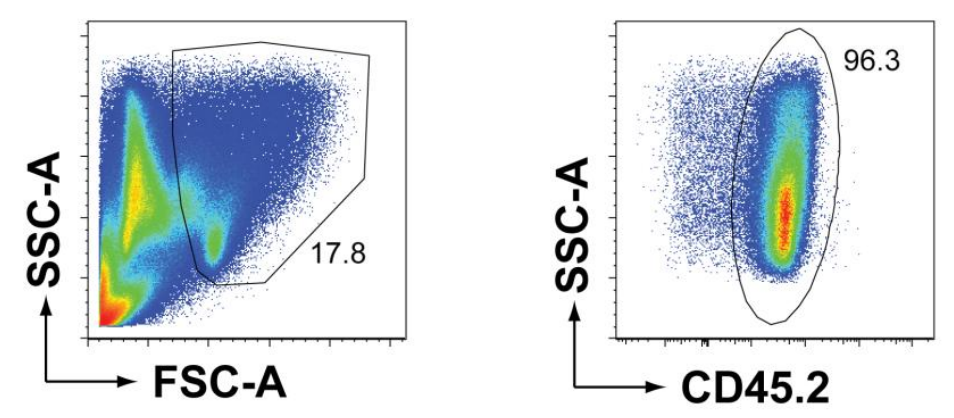

Dendritic cell subsets
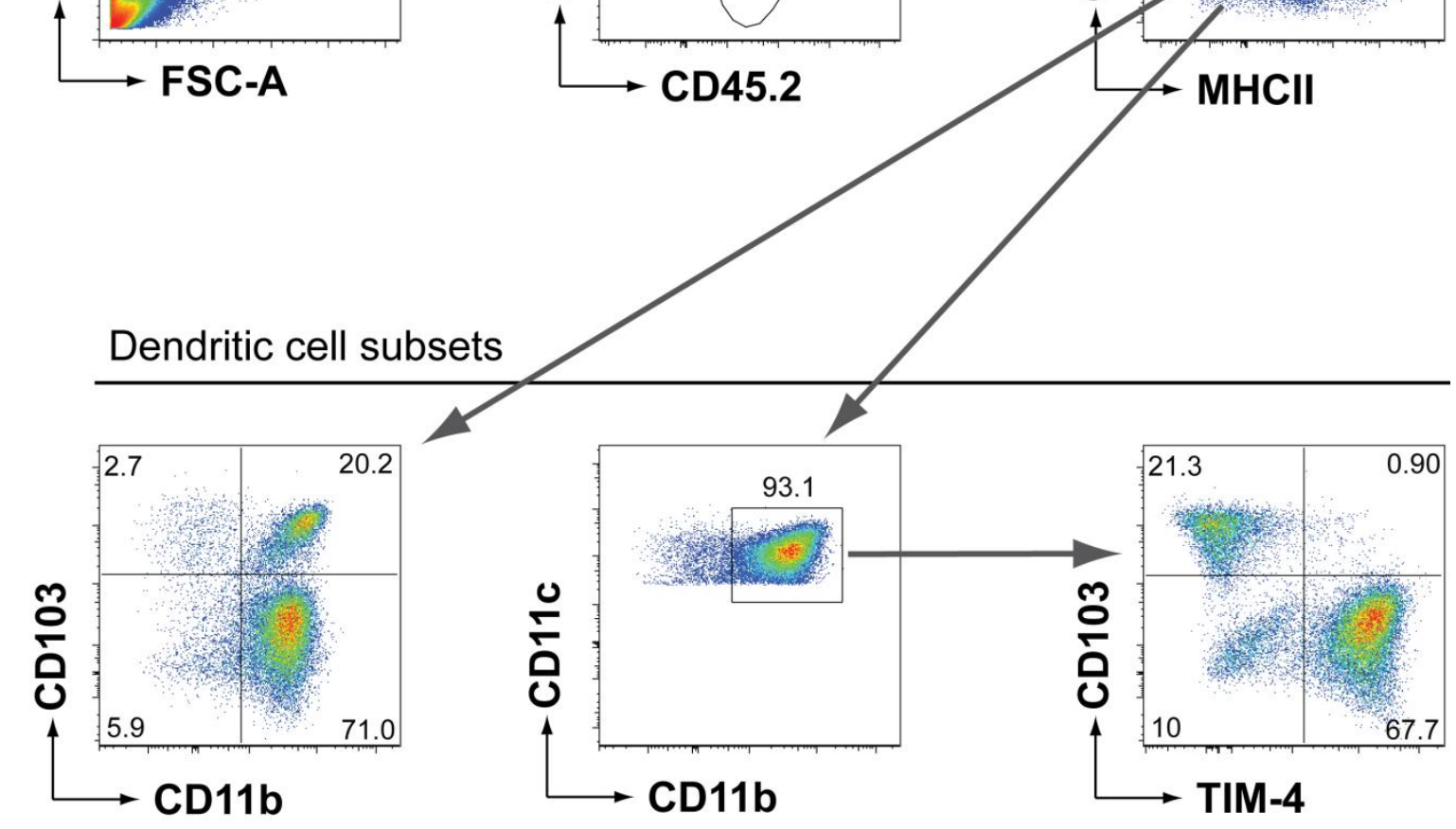

Appendix I: Gating strategy used to identify APC subsets in the jejunal LP 

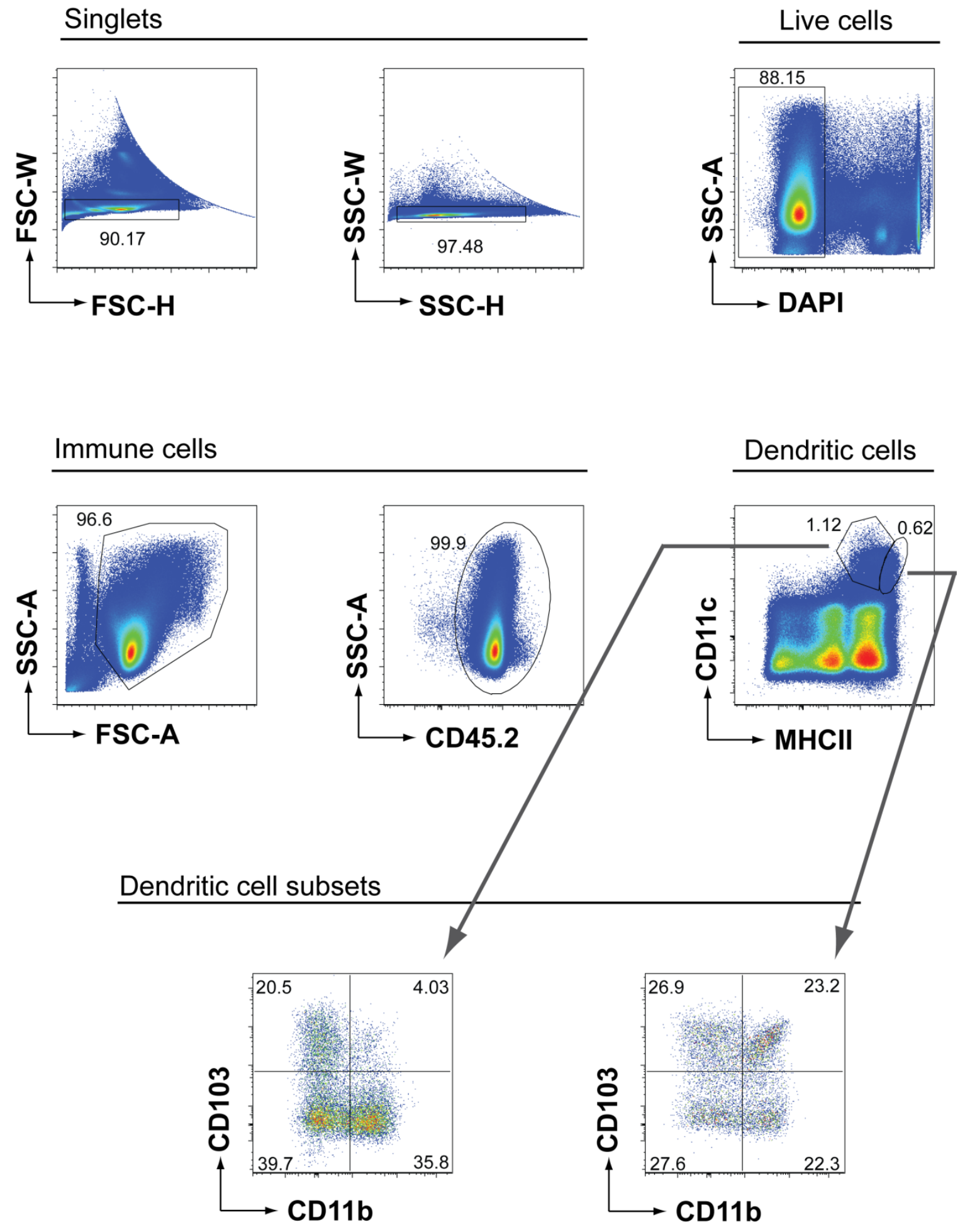

Appendix II: Gating strategy used to identify APC subsets in the MLN 